CLASSIFICATION AND MAPPING OF QUATERNARY SEDIMENTARY DEPOSITS FOR PURPOSES OF SEISMIC ZONATION, SOUTH COASTAL LOS ANGELES BASIN, ORANGE COUNTY, CALIFORNIA

David R. Fuller, Edward C. Sprotte, Richard B. Greenwood, Henry A. Mumm, Charles R. Real, Roger W. Sherburne

\author{
California Department of Conservation \\ Division of Mines and Geology \\ 1416 Ninth Street \\ Sacramento, California 95814
}

USGS CONTRACT NO. 14-08-0001-18241

Supported by the EARTHQUAKE HAZARDS REDUCTION PROGRAM

OPEN-FILE NO. 81-966

U.S. Geological Survey

OPEN FILE REPORT

This report was prepared under contract to the U.S. Geological Survey and has not been reviewed for conformity with USGS editorial standards and stratigraphic nomenclature. Opinions and conclusions expressed herein do not necessarily represent those of the USGS. Any use of trade names is for descriptive purposes only and does not imply endorsement by the USGS. 


\title{
Classification and Mapping of Quaternary Sedimentary Deposits for Purposes of Seismic Zonation \\ South Coastal Los Angeles Basin, Orange County, California
}

\section{$14-08-0001-18241$}

\author{
E.C. Sprotte, D.R. Fuller, R.B. Greenwood, \\ H.A. Mumm, C.R. Real, R.W. Sherburne \\ California Division of Mines and Geology \\ 1416 Ninth Street, Room 1341 \\ Sacramento, California 95814
}

Interim Report-Second Year of Investigation

Valid and meaningful estimations of the severity and extent of ground shaking in an area resulting from earthquake energy release, and the secondary effects which may occur as a result of this ground response, must necessarily consider the thickness, configuration and material properties of the unconsolidated near surface sediments effected. Included in this report are map displays of the historically shallowest ground water occurrences, the distribution and character of discriminated surficial sediments and thicknesses of Holocene and Quaternary age sediments.

The response of near surface sediments to seismically induced shaking is a function of sediment characteristics, materials properties and the velocities of seismic waves through these sediments. Downhole velocity surveys have been conducted in existing boreholes. Additionally, the drilling, logging, sampling for bulk density and the downhole measurement of $\mathrm{P}$-and $\mathrm{S}$-wave traveltimes in a 200 foot hole has provided a means of estimating the clastic properties of the near surface sediments. Because many of the available boreholes in the area of investigation are surrounded by asphalt it was desirable to determine what effect an asphalt layer at the ground surface introduces to the traveltime curve. At the location of the 200 foot hole drilled during this year's effort, it was possible to measure downhole traveltimes from a source generated on soil and from a source generated on asphalt. Comparison and analysis of S-wave traveltimes data from these two sources is presented with the conclusion that when the source is on asphalt the measured traveltime is less than the measured traveltime on soil at shallow hole depths. Based on this simple test, S-wave traveltime disparity diminishes with increasing depth and traveltime curves for energy sources on soil and asphalt closely approach each other. 
In order to expediently collect and expeditiously analyze the mass of subsurface data accumulated for this investigation an electronic data processing management program has been developed to store, selectively retrieve and graphically display accumulated stratigraphic sample data and geotechnical measurements taken from holes in the ground. The current system can only store point data, data representing a measured value at some point in space and time. The system does not preclude adding range data. The system consists of two major functions, an edit-audit-update function (input) and a data retrieval function (output). The input function assures that only data that is usuable and consistent with user specified requirements reaches the storage file. The output function provides as variety of services related to obtaining and displaying information from the storage file. The edit-audit-update function is driven by a user-coded input form, while the data retrieval function is driven by a user coded query. Description of the processing capability of this developed program is contained in the report. 
ANNUAL TECHNICAL REPORT TEXT AND PLATES

COVERING THE PERIOD SEPTEMBER 19, 1979 to SEPTEMBER 18,1980

CLASSIFICATION AND MAPPING OF QUATERNARY SEDIMENTARY

DEPOSITS FOR PURPOSES OF SEISMIC ZONATION, SOUTH

COASTAL LOS ANGELES BASIN, ORANGE COUNTY, CALIFORNIA

By: Edward C. Sprotte, Senior Geologist, Principal Investigator David R. Fuller, Geologist, Co-Principal Investigator Richard B. Greenwood, Geologist Henry A. Mumm, Senior Programer Analyst Charles R. Real, Seismologist Roger W. Sherburne, Senior Seismologist

CALIFORNIA DEPARTMENT OF CONSERVATION Division of Mines and Geology

1416 Ninth Street, Sacramento, California 95814

Date Submitted: November, 1980

Sponsored by: U.S. Geological Survey Contract No. 14-08-0001-18241

Technical Officer: Gordon W. Greene

Effective Date of Contract: September 19, 1979

Expiration Date of Contract: September 18, 1980

Amount of Contract: $\$ 85,000$

Investigation also sponsored by: County of Orange, California Environmenta 1 Management Agency Cooperative Agreement No. 5-0052

The views and conclusions contained in this document are those of the authors and should not be interpreted as necessarily representing the official policies, either expressed or implied, of the U.S. Govermment. 
Graphics by: Victor A. Protasov Richard T. Boylan

Stenographics by: Venice Huffman Sue E. Torres 


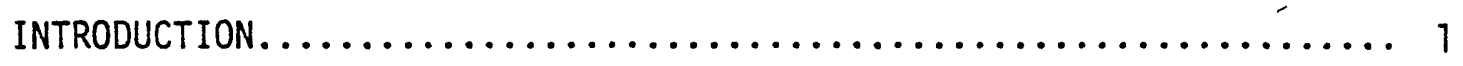

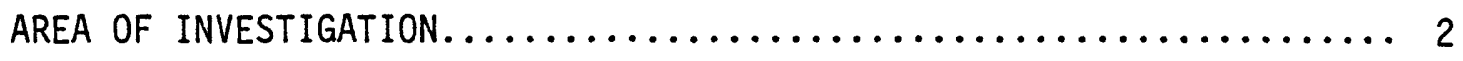

RESEARCH PROJECT OBJECTIVES ........................ 3

RESULTS OF THE SECOND YEAR OF INVESTIGATION.............. 5

ISOBATH MAP OF NEAR-SURFACE WATER - MAP NO. $1 \ldots \ldots \ldots \ldots \ldots \ldots \ldots \ldots$

SURFICIAL GEOLOGIC MAP OF QUATERNARY AGE SEDIMENTS - MAP NO. 2... 10

THICKNESS MAP OF HOLOCENE AGE SEDIMENTS - MAP NO. $3 \ldots \ldots \ldots \ldots \ldots \ldots$

THICKNESS MAP OF QUATERNARY AGE SEDIMENTS - MAP N0. $4 \ldots \ldots \ldots \ldots \ldots 14$

TALBERT AND BOLSA AQUIFERS MAP - MAP NO. $5 \ldots \ldots \ldots \ldots \ldots \ldots \ldots \ldots$

DOWNHOLE MEASUREMENT AND ANALYSIS OF P-WAVE AND S-WAVE SEISMIC

TRAVELTIMES RECORDED IN A WELL DRILLED IN MILE SQUARE PARK,

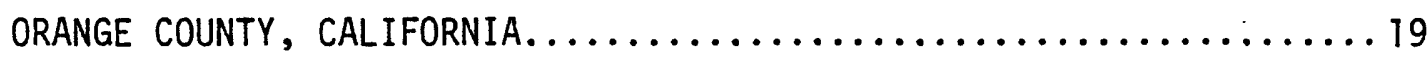

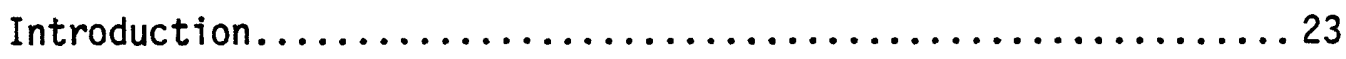

Seismic Methods................................. 24

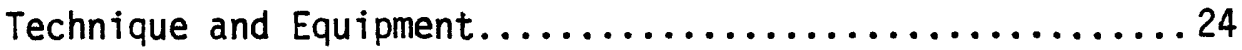

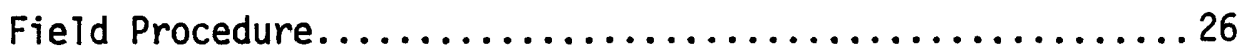

Results From Mile Square Park Borehole................. 29

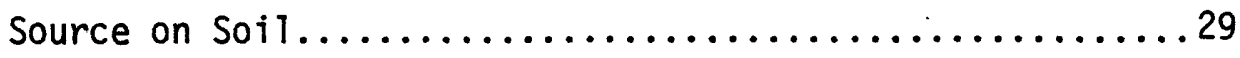

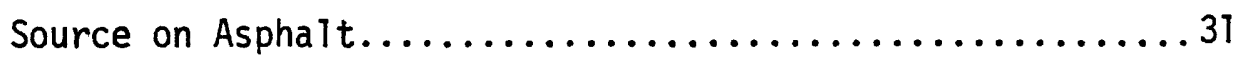

Comparison on Source on Soil with Source on Aspha1t......32

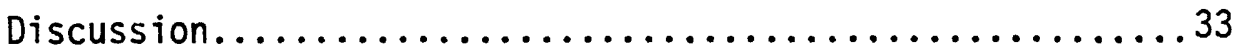

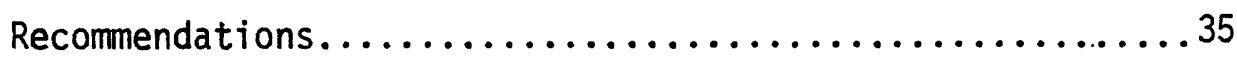

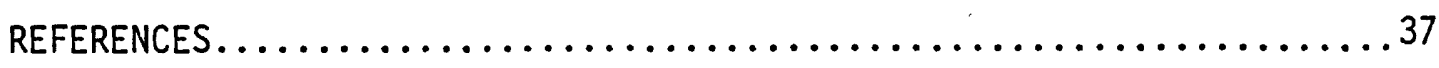


CONTENTS (Continued)

Page

SUPPLEMENT TO THE ANNUAL TECHNICAL REPORT DESCRIPTION OF THE

ELECTRONIC DATA PROCESSING CAPABILITY DEVELOPED TO MANAGE

STRATIGRAPHIC SAMPLE DATA AND GEOTECHNICAL MEASUREMENTS $\ldots \ldots \ldots \ldots 87$

LISTING OF MAPS AND PLATES

In Pocket

Map No. 1. Isobath map of near-surface water, portion of Orange County, California.

Plate 1. Whittier, La Habra, Los Alamitos and Anaheim quadrangles.

Plate 2. Yorba Linda, Prado Dam, Orange and Black Star Canyon quadrangies.

Plate 3. Seal Beach, Newport Beach and Costa Mesa quadrangles....

Plate 4. Tustin, El Toro, Laguna Beach and San Juan Capistrano quadrangles.

Map No. 2. Surficial geologic map of Quaternary age sediments, portion of Orange County, California...

Plate 1. Whittier, La Habra, Los Alamitos and Anaheim quadrangles.

Plate 2. Yorba Linda, Prado Dam, Orange and Black Star Canyon quadrangles.

P1ate 3. Seal Beach, Newport Beach and Costa Mesa quadrangles....

Plate 4. Tustin, El Toro, Laguna Beach and San Juan Capistrano quadrangles. 
CONTENTS (Continued)

In Pocket

Map No. 3. Thickness map of Holocene age sediments, portion of Orange County, California.

Plate 1. Whittier, La Habra, Los Alamitos and Anaheim quadrangles.

Plate 2. Yorba Linda, Prado Dam, Orange and Black Star Canyon

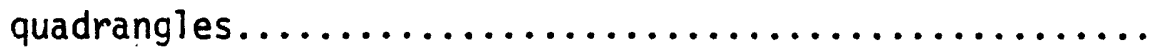

Plate 3. Seal Beach, Newport Beach and Costa Mesa quadrangles....

Plate 4. Tustin, El Toro, Laguna Beach and San Juan Capistrano quadrangles.

Map No. 4. Thickness map of Quaternary age sediments, portion of Orange County, California

Plate 1. Whittier, La Habra, Los Alamitos and Anaheim quadrangles.

Plate 2. Yorba Linda, Prado Dam, Orange and Black Star Canyon quadrangles.

Plate 3. Seal Beach, Newport Beach and Costa Mesa quadrangles....

Plate 4. Tustin, El Toro, Laguna Beach and San Juan Capistrano quadrangles.

Map No. 5. Talbert and Bolsa aquifers map, portion of Orange County, California.

Plate 1. Whittier, La Habra, Los Alamitos and Anaheim quadrangles. 
CONTENTS (Continued)

In Pocket

Plate 2. Yorba Linda, Prado Dam, Orange and Black Star Canyon quadrangles.

Plate 3. Seal Beach, Newport Beach and Costa Mesa quadrangles....

LISTING OF FIGURES

Page

Figure 1. Index map............................. 4

Figure 2. Sidewall clamping triaxial borehole geophone.........4 41

Figure 3. Twelve channel signal enhancement seismograph........ 41

Figure 4. Typical composite record showing recorded source

signals from normal and reversed horizontal S-wave impacts, and a vertical P-wave impact............42

Figure 5. Composite time histories of normal and reversed S-wave source polarity on soil at sample depths in the borehole as registered by both horizontal components. P-wave onset pulse as recorded by the vertical component is also shown $\ldots \ldots \ldots \ldots \ldots \ldots \ldots \ldots \ldots \ldots$

Figure 6. Traveltime-depth curves for P- and S-waves generated on soil showing detailed interpretation (Model 1).....44

Figure 7. P-and S-wave interval velocity-depth profiles computed from the finely interpreted traveltime breaks shown in Figure $6 \ldots \ldots \ldots \ldots \ldots \ldots \ldots \ldots \ldots \ldots \ldots \ldots \ldots \ldots \ldots \ldots \ldots \ldots$

vii (p. ix follows) 
CONTENTS (Continued)

Page

Figure 8. Composite time histories of normal and reversed $S$-wave source polarity on asphalt at sample depths in the borehole as registered by both horizontal components... 46

Figure 9. Traveltime-depth curve for S-wave generated on asphalt showing detailed interpretation. P-wave traveltime curve is for soil source....................47

Figure 10. S-wave interval velocity-depth profile computed from the interpreted traveltime breaks shown in Figure 9. Source on asphalt..................... 48

Figure 11. Traveltime-depth curve for $\mathrm{S}$-wave generated on asphalt interpreted over the same intervals as identified on the source traveltime curve in Figure 6 (Model 1).....49

Figure 12. S-wave interval velocity-depth profile computed from the interpreted traveltime breaks shown in Figure 12

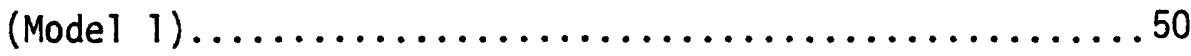

Figure 13. Coarse interpretation of soil S-wave traveltime curve

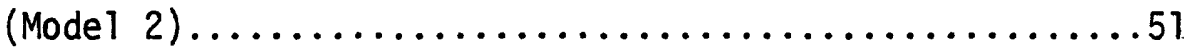

Figure 14. S-wave interval velocities from coarse interpretation of soil traveltime curve (Model 2).............52

Figure 15. Coarse interpretation of asphalt S-wave traveltime

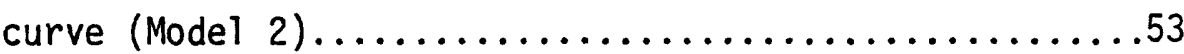

Figure 16. S-wave interval velocities from coarse interpretation of asphalt traveltime curve (Model 2)............54 
CONTENTS (Continued)

Page

Figure 17. Raypath for downhole velocity survey with source plank on asphalt.............................. 55

Figure 18. Comparison of traveltime curves for S-waves generated

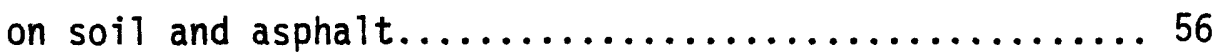


Introduction

The objective of this investigative research is the development of a scientifically valid analytical procedure enabling plausible prediction of the areal extent and severity of the dynamic responses of young loosely consolidated near surface sediments to seismically induced ground shaking. Specifically, the area of investigation under this research project is that portion of the south coastal Los Angeles basin within Orange County, California. This area is covered by unconsolidated and loosely consolidated Quaternary age sediments of varying thickness. Except for the brief historical record, how these young near surface sediments will respond to ground shaking resulting from earthquakes is largely undertermined.

This technical report presents the results achieved during the secondyear period of this continuing investigation. No. conclusions and no estimates of seismic ground response are included in this interim report. The results of the second-year of investigation which are presented include map displays of ground water occurrence, the distribution and character of discriminated surficial sediments and thicknesses of Holocene and Quaternary age sediments. During this report period a 200 foot hole was drilled, logged and sampled through Holocene age sediments and into underlying Pleistocene age sediments. With the use of a side wall clamping three-directional geophone and a multichannel signal enhancement recording seismograph, downhole seismic wave traveltimes from a ground surface origin were measured. Analyses of these seismic wave traveltimes are presented. 
Included as a supplement to this annual Technical Report is a Description of the Electronic Data Processing Capability Developed to Manage Stratigraphic Sample Data and Geotechnical Measurements. This data management program has been developed specifically to store, selectively retrieve and graphically display data collected for this investigation and other similar investigations which might be undertaken.

\section{AREA OF INVESTIGATION}

The area of this investigation is the southeastern portion of the Los Angeles basin within Orange County, California. This $900 \mathrm{~km}^{2}$ area of coastal plain has undergone rapid urban growth during the last two decades and currently contains an estimated 1.5 million inhabitants. Urban growth and resident population are projected to increase.

Orange County will be subject to the impact of large and possibly damaging earthquakes which may occur along any of the several major active and potentially active faults both within or in the proximity of the county. Seismic wave impact of the study area can reasonably be expected 
to occur from earthquakes originating from nearly every direction. Location of the area of this investigation in relation to Quaternary age faulting and epicenter locations of historic large earthquakes is shown on the the index map (figure 1) page.

The relatively flat-lying unconsolidated to loosely consolidated clastic sediments of Quaternary age which underlay the study area vary in thickness and have an indicated maximum thickness in excess of 4,000 feet near the center of the study area. How these sediments will respond to seismically induced ground shaking has not been previously analyzed on an areal basis. Estimation of the response of these sediments to seismic wave stimulation, and the areal distribution and severity of this response, is the purpose of this investigation.

\section{RESEARCH PROJECT OBJECTIVES}

The objective of this investigation is the development of a plausible and scientifically valid estimate of the type and relative severtiy of the dynamic response of young relatively unconsolidated near surface sediments to seismic wave impact. To achieve this objective a multiyear investigation has been undertaken in the Orange County study area.

In the first year of investigation available geotechnical data from engineering projects and other types of investigations which are applicable to a seismic ground response investigation were collected and spatially identified. Development of an electronic data processing program to manage data collected and to be collected for interpretation in this investigation was commenced. 


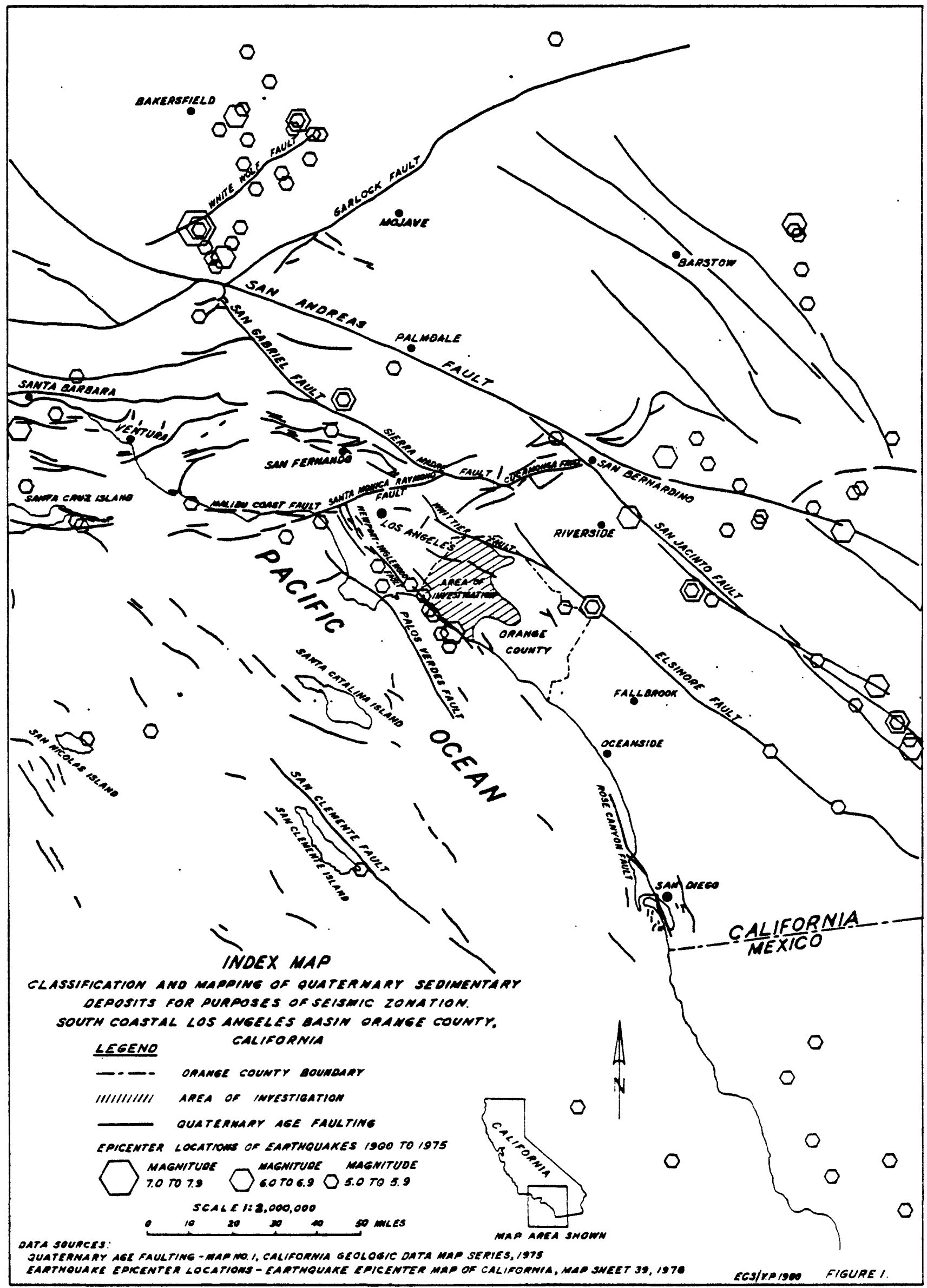


During this second year of investigation, data collected were interpreted to define the geometry and character of discriminated near surface sediments and the proximity of ground water to the ground surface. Additional data, primarily seismic velocity data, were collected through project field work in order that these seismic P-wave and S-wave velocity data could be related to other measured physical parameters of near surface sediments. Development of an electronic data processing management program to store, selectively retrieve and graphically display stratigraphic sample data and geotechnical measurements was completed.

Program objectives to be achieved during the third year of this investigation, commencing September 19, 1980, without additional project drilling and down-hole seismic velocity measurements, include map displays of the susceptibility of near surface sediments to liquefaction and differential settlement, the locations of reported historic seismic intensities and graphical and/or tabular displays of preliminary correlations among selected measured seismic and geologic parameters.

\section{RESULTS OF THE SECOND YEAR OF INVESTIGATION}

Results and products of the second year of this continuing investigations are contained in this Annual Technical report and include:

A. Map displays of:

1. The historically shallowest ground water occurrence below the ground surface, Map No. 1, 
2. The character and distribution of Quaternary age surficial sediments, Map No. 2,

3. The thickness of Holocene age sediments, Map No. 3,

4. The thickness of Quaternary age sediments, Map No. 4,

5. The distribution and thickness of the Talbert and Bolsa aquifers of Holocene age, Map No. 5

B. Downhole measurement and analysis of $P$ - and $S$-wave seismic traveltimes recorded in a well drilled in Mile Square Park, Orange County, California.

C. A description of the electronic data processing capability developed to manage stratigraphic sample data and geotechnical measurements. 
ISOBATH MAP OF NEAR SURFACE WATER - MAP NO. 1

The isobath map of near surface water provides a conservative interpretation of the historically shallowest depth to free water or high interstitial water saturation within the study area. The data base used in the preparation of this map is provided by observations recorded in the logs of investigative borings undertaken for construction projects subsequent to the year 1955 and drillers logs of wells drilled for water development subsequent to the year 1920. The occurrence and location of historic surface water was determined from an examination of U.S. Geological Survey topographic maps dated 1901 and later, prior to the extensive incursions of cultural development. Locations of investigative borings and wells are indicated on the map to provide a perspective of the distribution of subsurface data which are the basis for the interpretation presented; however to facilitate map clarity data values and observation dates are not shown on the map. The interpretation presented is biased toward the shallowest reported observations of ground water, a conservative interpretation. This map is intended to be a regional and general evaluation of "worst possible" ground water conditions for seismic ground response considerations and is not intended for and should not be used for site evaluation or for other purposes.

Within the area of investigation displayed on this map, geologic factors which effect the depth to ground water include the spatial distribution and juxtaposition of near surface sediments having contrasting textures, the regional hydraulic gradient, and local topography. Shallow 
ground water contours at the mouths of Santa Ana Canyon and Santiago Creek are the result of inflow and percolation of water into recent coarse clastic sediments in these areas (Plate 2). Similarly, the shallow ground water located along the western boundary of the County is related in part to water from the San Gabriel River distributary system (Plate 1). In the area west of the mouth of Santa Ana Canyon and beneath the City of Anaheim sparse historic ground water observations indicate a depth to ground water of 50 feet or more below the ground surface (P1ate 1). Surface and near surface recent sediments in this area are generally unconsolidated permeable sandy coarse clastics as shown on Map No. 2. Holocene sediments are thick beneath this area as shown on Map No. 3 and the Holocene age Talbert aquifer is thickest beneath this area as shown on Map No. 5. Surface elevations in the area of the City of Anaheim are 100 to 150 feet above mean sea level and surface sediments are probabily in hydraulic continuity with the Talbert aquifer system. These conditions result in a deep ground water level. To the west of the City of Anaheim beneath the cities of Buena Park and Los Alamitos (Plate 1), different conditions exist and shallow ground water is indicated. Surface and near surface sediments are finer grained and probabiy less permeable than the sediments beneath Anaheim. Surface elevations are less than 75 feet above sea level so that the hydraulic gradient is less than in the Anaheim area. The mesas immediately inland from the seacoast àre composed of consolidated Pleistocene sediments and stand 50 to 100 feet above sea leve1. Ground water is 30 feet or more below the surface of these mesas (Plate 3$)$. In the near sea level gaps between these mesas, ground water is 3 feet or less below the ground surface in fine grained Holocene sediments. 
Inland from the coastline mesas and beneath the cities of Westminster, Fountain Valley, and Irvine, ground water is 5 . feet or less below the ground surface in unconsolidated Holocene sediments (Plates 1, 3, 4). Early topographic maps indicate marshy areas inland from these mesas. The consolidated Pleistocene age sediments on and beneath the mesas apparently have lower water transmissibility than the Holocene sediments and water is impounded against the uplifted Pleistocene sediments of the mesas.

The subsurface depth of interstitial water saturation in nearsurface sediments is a critical factor in the estimation of the effects, particularly the secondary effects of liquefaction and differential settlement, that may result from earthquake impact to these sediments. A determination of the historically shallowest occurrence of free ground water establishes the minimum depth limit for this factor. This is the purpose of this map interpretation. 
SURFICIAL GEOLOGIC MAP OF QUATERNARY AGE SEDIMENTS - MAP NO. 2

This map characterizes in a general manner, surface materials according to predominate grain size, consolidation and, in part, depositional environment. Data base for the preparation of this map is largely taken from the U.S. Department of Agriculture, Soil Conservation Service, 1976 Interim Report, "Soil Conservation Service, 1976 Interim Report, "Soil Survey of Orange and Western Part of Riverside Counties." The 226 soil units contained in this soil survey were reduced to the eight general descriptive units displayed on this map. NOTE: A map unit 1 designation has not been used in order to enhance map display clarity.

Map units 2, 3 and 4 are predominately unconsolidated sand, silt and clay units, respectively, of Holocene age. "These units occur in the central portion of the study area and in the topographic gaps between the coastal mesas, within the limits displayed on the Holocene thickness map, Map No. 3.

Map units 5, 6 and 7 are predominately consolidated sand, silt and clay units, respectively, of Pleistocene age. These units occur on the mesas near the coastline and generally at the northern, eastern and southeastern edges of the study area.

Units 8 and 9 are unconsolidated laqoonal-intertidal muds and beach deposits of sand and gravel, respectively, of Holocene age. These units occur along in proximity to the coastline. 
This map will provide information contributary to the estimation of the susceptibility of near surface sediments to liquefaction and differential settlement during seismically induced ground shaking. The map may also provide some correlations between soil character and documented seismic intensities at sites within the study area. 
THICKNESS MAP OF HOLOCENE AGE SEDIMENTS - MAP NO. 3

This map displays the interpreted total thickness of Holocene sediments within the area of this investigation. Thickness values used in preparing this map have largely been derived from lithologic descriptions contained in water well drillers logs and the descriptions of sediments penetrated in other types of investigative borings.

Basal Holocene sediments, in the area generally west of the present restricted course of the Santa Ana River and particularly from the mouth of Santa Ana Canyon southerly to the seacoast, are commonly characterized by conglomeratic gravelly coarse sands. This persistent occurrence of coarse clastic materials is largely the basis for the Holocene thickness interpretation shown on the map in this area. Holocene sediments easterly from the present coarse of the Santa Ana River are generally 20 feet, or less, in thickness and are mostly soils and sediments derived from underlying or nearby outcropping older sedimentary and volcanic rocks. Recognition of these older sediments in the lighologic descriptions of water wells and investigative borings is the basis for the Holocene thickness interpretation shown on the map in this area.

Holocene sediments are thickest on a trend, two to six miles wide, from the mouth of Santa Ana Canyon southerly to the seacoast between Newport and Huntington Mesas and, to a lesser degree of thickness, between Huntington and Bolsa Chica Mesas. This area of thick Holocene sediments is beneath the cities of Anaheim, Gardon Grove, Fountain Valley and, to a lesser degree of thickness, Westminster (plates 1, 2 
and 3 ). This maximum thickness trend is generally coincident with the areal distribution of the Talbert and Bolsa aquifer systems displayed on Map No. 5. In the western portion of the study area (Plates 1 and 3) a thick lobe of Holocene sediment extends southerly to the seacoast between Bolsa Chica Mesa and Landing Hill; this area of thick Holocene sediment is beneath the city of Los Alamitos and the U.S. Naval Weapons Station.

The distribution, thickness and character of unconsolidated to loosely consolidated Holocene sediments will be a determining factor in estimating the primary and secondary effects of ground shaking resulting from earthquakes. This map will provide a basis for defining the character of Holocene sediments and ultimately in estimating ground surface response to seismic loading. 
THICKNESS MAP OF QUATERNARY AGE SEDIMENTS - MAP NO. 4

This map displays the interpreted areal distribution and thickness of Quaternary Age sediments within the area of this investigation, that portion of the south coastal Los Angeles basin which is in Orange County. Areal extent of exposed Quaternary Age sediments has been taken from published information and is generalized. Subsurface interpretation of the thickness of Quaternary Age sediments is based largely on available information from deep wells drilled for oil and gas exploration and development. Geophysical logs from these wells were correlated to determine the base of Pleistocene sediments and the indicated thickness value from the ground surface to the correlated well depth was used to prepare this map display. Locations of these control wells are shown on the map. Well identities and data values used have been omitted in order to enhance map clarity. For purposes of this seismic ground response investigation thickness values used for this interpretation are gross vertical thickness values and should not be regarded as true stratigraphic thickness values.

Significant variation in the thickness of Quaternary age sediments occur within the study area and these thickness variations are displayed on the map. Quaternary Age sediments have a local maximum thickness of about 1,600 feet beneath the cities of La Habra and Brea (Plate 1). Sediments thin in a southerly direction to 600 feet beneath West Coyote and East Coyote Hills, Plate 1, and progressively thin in an easterly direction to onlap termination against older sediments on the flank of the Puente Hills east of the city of Yorba Linda and the western flank 
of the Santa Ana Mountains east of the city of Orange (P1ate 2). South of the Coyote Hills these sediments increase in thickness to about 4,000 feet beneath the city of Anaheim (P1ates 1 and 2) and reach an interpreted maximum thickness of 4,200 feet beneath the cities of Stanton and Garden Grove (Plate 1). From this maximum thickness, sediments uniformly thin in a southeasterly direction, beneath the cities of Santa Ana, Tustin and Irvine and extend thinly beneath the Tustin Plain to onlap contact with older sediments and volcanic rocks (Plates $1,2,3$, and 4 ). Southwesterly from this maximum thickness of 4,200 feet, Quaternary sediments thin uniformly toward the seacoast (Plate 3 ). Near the coastline, along the Newport Inglewood structural trend, Quaternary sediments thin to 500 feet beneath the city of Seal Beach, 800 feet beneath Sunset Beach, 600 feet beneath the city of Huntington Beach and terminate across Newport Mesa by onlap contact with older sediments. Extension of Quaternary age sediments beyond the limits of this investigation, offshore and within Los Angeles County is apparent but has not been defined by this study.

This map provides definition of the lateral distribution and thickness variation of young relatively unconsolidated sediments which will be subjected to earthquakes. Any estimation of the response of nearsurface sediments to earthquake energy release, and the primary and secondary effects which may occur, must consider the thickness and configuration of the effected sediments. 
TALBERT AND BOLSA AQUIFERS MAP - MAP NO. 5

This interpretive map displays the onshore limits of effective sand development of this aquifer system, structural contours on the top of these sand and gravel units and gross thickness values of these aquifers. The Talbert aquifer is the larger of these two units in length, thickness and areal extent. It extends from the mouth of Santa Ana Canyon to the seacoast between Huntington and Newport Mesas. In Santa Ana Canyon this sand and gravel unit is not readily distinguishable from overlying and underlying coarse clastic sediments of similar character. This aquifer has a width of about two miles and a thickness of about 20 feet at the mouth of Santa Ana Canyon. The aquifer expands rapidly to a width of about six miles and has a maximum gross thickness of about 90 feet beneath portions of the cities of Garden Grove, Westminster and Fountain Valley. The Talbert aquifer narrows south of the city of Fountain Valley to a width of about two miles and extends to the coastline through Santa Ana Gap, between Huntington and Newport Mesas. Maximum thickness of the Talbert aquifer at the coastline is 90 feet and seaward extension of the aquifer is indicated but has not been delineated for purposes of this investigation.

South of the city of Westminster and north of Huntington Mesa the aquifer system bifurcates and the smaller shorter Bolsa aquifer extends to the seacoast between Huntington Mesa and Bolsa Chica Mesa. The Bolsa aquifer is in hydraulic continuity with the upper youngest portion of the Talbert aquifer. Both aquifers appear to be similar in lithologic character and are of Holocene age. For the purposes of this 
investigation these contiguous sand units have been mapped as a single discriminated lithologic unit of Holocene age.

This combined sand unit constitutes a significantly large segment of the total Holocene sediment thickness within the area of this investigation. Adequate considerations of the extent, thickness and depth below the surface of this discriminated sand unit will be essential in estimating the primary and secondary ground surface effects resulting from seismic impact of this area. 
DOWNHOLE MEASUREMENT AND ANALYSIS OF

P- AND S-WAVE TRAVELTIMES RECORDED IN A WELL DRILLED

IN MILE SQUARE PARK, ORANGE COUNTY; CALIFORNIA

By

Charles R. Real and Roger W. Sherburne

19 (p. 21 follows) 
CONTENTS

Page

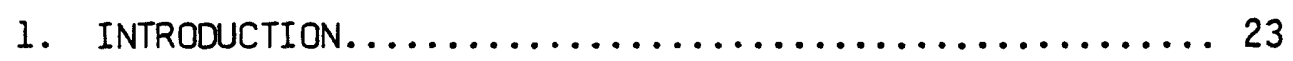

2. SEISMIC METHODS....................... 24

Technique and Equipment............... 24

Field Procedure.................... 26

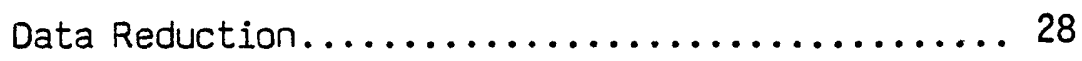

3. RESULTS FROM MILE SQUARE PARK BOREHOLE............. 29

Source on Soil....................... 29

Source on Asphalt................... 31

Comparison, of Source on Soil with

source on asphalt................... 32

4. DISCUSSION........................... 33

5. RECOMMENDATIONS...................... 35

6. REFERENCES.......................... 37

21 (p. 23 follows) 
DOWNHOLE MEASUREMENT AND ANALYSIS OF

P- AND S-WAVE TRAVELTIMES RECORDED IN A WELL DRILLED IN

MILE SQUARE PARK, ORANGE COUNTY, CALIFORNIA

\section{INTRODUCTION}

The response of near surface geologic deposits to seismically induced shaking is a function of material properties including density, stiffness, fluid content, porosity, shear modulus, bulk modulus and layer geometry. Particular combinations of these properties can selectively amplify a narrow frequency band of ground motion while reducing motion at other frequencies. Measurement of P- and S-wave seismic velocities and laboratory determinations of bulk density provide a means of estimating the elastic properties of the near surface materials. Unfortunately, among the available geotechical data collected thus far for the Orange county study area, the distribution of seismic velocities is least known. Consequently, a program task was developed to conduct downhole velocity surveys in existing boreholes, as well as to conduct shallow drilling and velocity measurements in selected areas to improve subsurface control of important physical parameters. 
This section describes the method employed in the measurement of seismic velocity, the resolution attained, and the results from a survey of Mile Square Park borehole (MSP-1) drilled as part of this years investigations (Map 5, Plate 3). Velocity data obtained from five water investigation wells and two engineering borings surveyed this year are pending reduction and are not included in this report.

Because many of the available boreholes are surrounded by asphalt, it was desirable to determine what effect an asphalt layer introduces to a traveltime curve. The MSP-1 borehole afforded a unique opportunity to evaluate this affect because the nole was drilled adjacent to a parking lot so that at a 10 foot offset distance, the source could be generated on asphalt on one side of the borehole, or on soil on the sther side. Results of this test are included in this section.

\section{SEISMIC METHODS}

Technique and Equipment

Downhole tecnniques were used for the determination of soth $\mathrm{P}$ - and S-wave velocity, wnereby a surface source generates a wave that travels downward through the substratum and is recorded by a sensor in a bore- 
hole. The P-wave source consists simply of a vertical impact on the ground surface produced by a sledgehammer blow. The S-wave source energy is generated by the "plate and hammer" or "horizontal traction" method introduced by Japanese researchers (Kobayasni, 1959; Kudo and Shima, 1970) and successfully used to measure shear-wave traveltimes in ialifornia (Warrick et al., 1974; Power and Real, 1976). The technique consists of impacting the end of a traction plank held firmly to the ground by the weight of a vehicle, thereby generating a tangential stress wave that travels vertically into the ground. The method has been described in detail by Beeston and McEvilly (1977).

The borehole sensor used in this study was a Mark Products L-10-30 SWC sidewall clamping geophone (figure 2) incorporating three or thogonally mounted transducers, each naving a natural frequency of $4.5 \mathrm{~Hz}$. The geophone is held firmly in place by a bowspring clamp whicn triggers on bottom impact and will operate in boreholes ranging from 3.0 to 6.0 inches in diameter, when used with appropriate spacers. The tool is suspended in the borehole by a 5/16 inch steel stress caole having moided deptn markers at 5 foot intervals to facilitate proper positioning. The transducer signals are carried to the recorder via a separate sinielded multi-conductor cable.

The geophone signals are conditioned and recorded by a Geometrics ES-1210 12 channel digital signal enhancement seismograph (figure 3). Signal ennancement is achieved by the metnod of vertical stacking, wheredy 
the signals from several successive source impacts are summed and stored in memory. Coherent energy (the wave arrival) adds constructively and random energy (ground noise) tends to cancel. Tine method improves the signal to noise ratio by a factor of $\sqrt{n}$ for $n$ impacts, thereby permitting wave interpretations to greater depths. Each geophone component is displayed on four channels wich permits up to four independent gain settings and stacking combinations for each component. While conducting a measurement, all channels are displayed on a CRT soreen for visual monitoring. When a satisfactory signal is attained, a permanent record is obtained from a non-photographic thermal printer.

Field Procedure

The routine field procedure oegins with the lowering of a "fish-tool" to detect possible down hole obscructions and verify the depin of the borehole. This precautionary measure was taken primarily vecause many of the boreholes to be surveyed are pre-existing water investigation wells with perforated casing that can deform under stresses induced by soil creep over long periods. The "fish-tool" also served to test the firmness of the hole bottom; a firm hole bottom is required to activate the trigger foot at the base of the geophone to release the bowspring sidewall clamp. When the bottom material appears too soft, several small coboles are dropped down the hole and compacted with the "fisi-tool" to provide a firm surface for the trigger foot. 
The routine source configuration for a downhole velocity survey is a five foot offset from the borehole for $P$-wave impacts, and a ten foot offset for the S-wave traction plank impact. When the geophone has reached bottom and the sidewall clamp has properly activated, P- and S-wave traveltime is measured and the tool is pulled to the nearest five foot marker. From this point to the surface, the borehole is logged at five foot intervals.

At each sample point, horizontal components $\mathrm{H} 1$ and $\mathrm{H} 2$ are recorded on channels one and five for a left side impact, and on channels two and six for a right side impact (as determined facing toward the front of the vehicle in the drivers seat), during measurement of S-wave traveltime. This arrangement conveniently places the right and left side impact traces for each horizontal component adjacent to one another for comparison. The $\mathrm{P}$-wave source signal is recorded by horizontal component $\mathrm{H} 2$ on channel eight, and the vertical component on channel twelve. In most instances, the $\mathrm{H} 2$ component on channel eight records a distinct S-wave phase (probably SV) generated by the P-wave source impact. With the described recording channel configuration, a complete set of $\mathrm{P}$ - and S-wave traveltime measurements for a single sample point is contained on a single record. Figure 4 shows a typical permanent record obtained at a single sample point in the Mile Square Park borehole. 
Data Reduction

When a suite of records has been obtained from a borehole survey, the usual procedure is to first prepare a tracing of the time histories such that at each sample point, the right and left side impacts are superposed. Because of the reversal of S-wave source polarity, the superposed traces should be 180 degrees out-of-phase. The S-wave onset is then identified at the first point of trace divergence. Using this technique, the arrival times are read independently from both horizontal components. The $P$-wave onset is read from the vertical component trace.

As shown in figure 4, the high resolution timing lines on a $500 \mathrm{~ms}$ record are $5 \mathrm{~ms}$ apart, consequently the arrival time is. read to the nearest millisecond. A routine analysis of reading errors has shown tnat at this playback setting, the standard deviation of arrival time for replicate readings by different observers is about $0.8 \mathrm{~ms}$ for S-wave and $0.7 \mathrm{~ms}$ for P-waves. The F-test shows these values are not significantly different at the 95 percent confidence level. Thus, the reading uncertainty for P- and S-wave arrival time is slightly less than $1 \mathrm{~ms}$. It will be shown that this is not sufficiently precise to determine the value of high velocities for thin beds.

Traveltime curves are constructed for each component and are used to identify bad picks on the seismic records. Finally, a traveltime curve is constructed from the average arrival time of the horizontal components at 
each sample point corrected for vertical travel. Changes in slope are identified on this curve, and straight line segments are fit to the data by the method of least squares. The slopes of these lines are the average velocities of the corresponding depth intervals.

\section{RESULTS FROM MILE SQUARE PARK BOREHOLE SURVEY}

Source on Soil

Figure 5 shows the composite time histories for the S-waves, and the P-wave onset pulse for the source on soil at the MSP 1 borehole. The S-wave arrivals are less than 180 degrees out-of-phase from the surface to a deptin of about 35 feet for the $\mathrm{Hl}$ component and about 15 feet for the $\mathrm{H} 2$ component. Beyond these depths, the S-wave arrivals appear to indeed be phase shifted about 180 degrees. Near the point of arrival, the traveltime for the $\mathrm{P}_{-}$and $\mathrm{S}$-waves is noted on figure 5 . Slight rotation of the borehole tool as it travels up the hole is evident by a decrease in amplitude on one component and a corresponding increase in amplitude on the other. The nigher amplitude is recorded on the component more parallel to the source traction plank. For this reason, traveltime is computed from the average of the horizontal components.

During an initial test, an intermediate arrival between the P- and the S-wave was clearly recorded that seriously obscurred the onset of the 
S-wave. This intermediate phase looked similar to the "tube waves" observed by Beeston and McEvilly (1977). We found this phase to be a wave traveling down the borehole geophone cables when under tension. When the tension was relieved, and the cables allowed to fall back into the borehole, this phase completely disappeared.

Traveltime-depth curves for $\mathrm{P}$ - and S-wave arrivals are shown in figure 6. For comparison, the lithologic log for MSP-1 is included with all traveltime curves and velocity profiles. Particularly noticeable on tine $P$-wave curve is the constant traveltime over several depth intervals, which would correspond to infinite velocity. There is even a traveltime reversal at 60 feet. These features projably result from tne limited resolution of the recordings at the $500 \mathrm{~ms}$ record lengtn setting. The traveltime of a wave passing through a 5 foot layer having a velocity of $5000 \mathrm{ft} / \mathrm{sec}$ is only $1 \mathrm{~ms}$. The reading error is estimated to je slightly less than $1 \mathrm{~ms}$; however, considering propagation of errors for a traveltime difference increases this uncertainty by a factor of $\sqrt{2}$. Thus, the error in $\Delta T$ is over $1 \mathrm{~ms}$. Higher velocities will produce indistinguishable traveltime differences over even greater depth intervals. Consequently, a higher resolution recording is necessary to resolve 5 foot beds having velocities greater tnan about $5000 \mathrm{ft} / \mathrm{sec}$, and the $P$-wave traveltime curve in figure 6 has been interpreted at a lower resolution than desired. 
The traveltime-depth curve for the S-waves appears much smoother because S-wave velocity is muci lower corresponding to greater traveltime and a smaller percentage error. The velocity-depth profiles corresponding to both the P- and S-wave traveltime curves is shown in figure 7.

Traveltime breaks, for S-waves, appear to generally correspond to changes in lithology. The most pronounced oreak occurs at about 60 feet and corresponds to the transition between a predominantly sand-clay section above and a sand-gravel section below (the Talbert alquifer). S-wave traveltime seems to be sensitive to lithologic changes over intervals as small as 10 feet as shown by the increase in velocity between 35 and 40 feet corresponding to the absence of peat.

\section{Source on Asphalt}

Figure 8 shows composite time histories for S-waves with annotated arrival times for the source plank on asphalt. The S-wave arrivals appear to be inverted 180 degrees below about 30 feet on the $\mathrm{Hl}$ component and 10 feet on the $\mathrm{H} 2$ component. The traveltime curve of the average arrival times with interpreted breaks is shown in figure 9, and the corresponding velocity depth profile is shown in figure 10. 


\section{Comparison of Source on Soil with Source on Asphalt Results}

There are considerable differences'between the results from the soil and asphalt source. Comparison of figures 6 and 9 show that the difference in traveltime to common depth points is not constant between the two surveys. The average difference is about $3 \mathrm{~ms}$ sooner for the asphalt survey, with a standard deviation of about $2 \mathrm{~ms}$. Correspondingly, slopes over common depth intervals differ by as much as 50\%. The most dramatic difference occurs at a depth of about 100 feet. The resulting velocities are correspondingly different (figures 7 and 10). Traveltime breaks occur at different depths as well, so it can be concluded that for a high resolution interpretation, the soil and asphalt S-wave traveltimes widely differ.

For a more direct comparison, interval velocities were computed from the asphalt traveltime data over the same depth intervals as defined on the soil source survey (model 1). The results are shown in figures 11 and 12 and are summarized in table 1 . In general, the greatest differences occur over small intervals and may result in part from a combination of instrumental and reading errors.

Figures 13-16 and table 2 summarize the results of a coarse interpretation (model 2) of the soil and asphalt source traveltime curves. It is 
clear that the resulting interval velocities are in much closer agreement; within 5\% over most of the intervals. The large excursion (31\%) over the interval between 95 and 115 feet has not yet been explained.

\section{DISCUSSION}

There are three possible sources contributing to the discrepency between the traveltimes observed in a jorehole from a source on soil and a source on asphalt: 1) reading errors, 2) instrumental errors, and 3) differences introduced by the asphalt layer. Reading errors have already been discussed, and are slightly greater than $1 \mathrm{~ms}$ for $\Delta T$. Because of their random nature, reading errors would not be expected to cause a constant difference in slope over several sample points.

Instrumental errors can be either random or systematic; however, the most probable instrumental error is in defining the origin time, caused by a random variation in response of the impact trigger. A solid state hammer switch is used, and was observed to even pre-trigger by a sudden jerk. The trigger response is not instantaneous, and undoubtably has some statistical variation in response time that depends on the character of the impact between the hammer and the plank. The magnitude of this variation is not yet known, but is probably. less than $1 \mathrm{~ms}$. This variation is reduced, however, by signal enhancement whereby a mean origin time of several successive impacts is effectively determined. The errors introduced by the trigger response can be eliminated by placement of a geophone 
on the surface, differencing its arrival time from the borehole arrival time (thereby eliminating the variation in trigger time common to both), and then correcting to true origin time by applying a static correction determined by the average of the ground geophone arrival times for the entire survey as discussed by Gibbs and others (1980). Like reading errors, the random nature of trigger response would probably not cause a constant difference in slope over several sample points.

The presence of an asphalt layer can introduce systematic changes in traveltime. Asphalt has a higher $\mathrm{P}$ - and $\mathrm{S}$-wave velocities than the loose near surface soil. Usually, in the preparation for paving, the ground is compacted, and a layer of gravel placed between the ground and the asphalt. This, in effect, can produce a higher velocity surface layer a few feet in thickness. Seismic waves produced on the surface of the asphalt will travel predominatly horizontally along this layer and be refracted into the ground at the critical angle defined by the inverse sine of the ratio of the higher velocity surface layer to the velocity of the substratum (figure 17). As can be seen by the three raypaths shown in figure 17, the distance traveled through the higher velocity surface layer is progressively shorter for measurements at greater depths in the borehole. This effect systematically increases traveltime with depth beyond the rate determined by the ambient velocity. The resulting effect is to decrease the slope of the traveltime curve, thereby underestimating seismic velocity when the source is on asphalt. The effect diminshes with depth, so 
that the traveltime curves for soil and asphalt approach one another. Figure 18 confirms this is indeed the case.

\section{RECOMMENDATIONS}

From this study, a number of conclusions can be drawn. It appears. that the resolution of the downhole technique using the equipment and procedures as described herein is limited to estimating the P- and S-wave velocities to within 5\% of beds no thinner than about $15-20$ feet. The resolution and reliability of velocity measurements can be improved by 1 ) reducing reading errors, 2) using a higher resolution recording (possible with the existing equipment), and 3) improve the accuracy of borehole measurements through asphalt by developing a correction procedure for its effect. Also noteworthy is that the entire data reduction procedure is slow and tedious and, if a production mode of operation were to be implemented, steps would have to be taken to expedite the process. The following are some specific recommendations directed toward meeting these needs.

1. Future downhole surveys should attempt a higher recording resolution. A $100 \mathrm{~ms}$ record has a $1 \mathrm{~ms}$ interval between the high resolution timing lines permitting reading to the nearest $0.1 \mathrm{~ms}$. The signal would be stretched, however, and the degree of resolution will be limited by how impulsive the arrival is. This should be tested during the next scheduled survey. 
2 A surface geophone arrival time should be used to difference from the borehole arrival times to eliminate origin time scatter caused by any random variations in response of the trigger.

3. The P- and S-wave velocity of the asphalt (and compacted) layer should be determined at each site, and a procedure developed to correct traveltimes for its effect.

4. A digital field tape recorder should be acquired, and a procedure developed for interactive picking and analysis of borehole velocity records. This process would permit more accurate reading of arrival time, and greatly speed up the entire data reduction process for a borehole velocity survey. 


\section{REFERENCES}

Beeston, H.E. and T.V. McEvilly, 1977, Shear wave velocities from downhole measurements: Earthquake Engineering and Structural Dynamics, v. 5 , p. 181-190.

Gibbs, J.F., T.E. Fumal, and E.F. Roth, 1980, In-situ measurements of seismic velocity at 27 locations in the Los Angeles, California Region: U.S.G.S. Open-File Report 80-378, 167 p.

Kobayashi, N., 1959, A method of determining the underground structure by means of SH wave: Zisin, Tokyo, Japan, v. 12, p. 19-24 (in Japanese).

Kudo, K and Shima, E., 1970, Attenuation of shear waves in soil: Bulletin of the Earthquake Research Institute of Tokyo, Japan, v. 48, p. 145-158.

Power, J.H., and C.R. Real, 1976, Shear wave velocity-propagation and measurement: California Geology, v. 29, n. 2, p. 27-29.

Warrick, R.E., 1974, Seismic investigations of a San Francisco Bay mud site: Bull. Seism. Soc. Am., v. 64, p. 375-385. 


\section{TABLES}

1. Comparison of S-wave velocities measured from a source on soil and on asphalt; fine interpretation (Model 1).

2. Comparison of S-wave velocities measured from a source on soil and on asphalt; coarse interpretation (Model 2). 
Table 1. Comparison of S-wave velocities measured from a source on soil and on asphalt; fine interpretation (Model 1).

\begin{tabular}{|c|c|c|c|c|c|c|}
\hline DEPTH & DEPTH & SOIL & ASPHALT & & & LAYER \\
\hline TOP & Воттом & vs & vS & $\Delta \mathrm{VS}$ & $\%$ & THICKNESS \\
\hline (FEET) & (FEET) & (FT/SEC) & (FT/SEC) & (FT/SEC) & DIFF & (FEET) \\
\hline 0 & 5 & 621 & 621 & 0 & 0 & 5 \\
\hline 5 & 20 & 379 & 432 & 53 & 14 & 15 \\
\hline 20 & 35 & 589 & 598 & 9 & 2 & 15 \\
\hline 35 & 45 & 751 & 704 & -47 & -6 & 10 \\
\hline 45 & 60 & 645 & 601 & -44 & -7 & 15 \\
\hline 60 & $85^{\circ}$ & 959 & 970 & 11 & 1 & 25 \\
\hline 85 & 100 & 876 & 1108 & 232 & 26 & 15 \\
\hline 100 & 105 & 2426 & 991 & -1435 & -59 & 5 \\
\hline 105 & 125 & 1179 & 1223 & 44 & 4 & 20 \\
\hline 125 & 135 & 2190 & 1242 & -948 & -43 & 10 \\
\hline 135 & 150 & 1243 & 1362 & 119 & 10 & 15 \\
\hline 150 & 165 & 1402 & 1327 & -75 & 5 & 15 \\
\hline 165 & 170 & 998 & 1658 & 660 & 66 & 5 \\
\hline 170 & 180 & 1808 & 1108 & -700 & -39 & 10 \\
\hline 180 & 190 & 1246 & 1246 & 0 & 0 & 10 \\
\hline
\end{tabular}


TABLE 2. Comparison of S-wave velocities measured from a source on soil and on asphalt; coarse interpretation (Model 2).

$\begin{array}{clccccc}\text { DEPTH } & \text { DEPTH } & \text { SOIL } & \text { 'ASPHALT } & & & \text { LAYER } \\ \text { TOP } & \text { BOTTOM } & \text { VS } & \text { VS } & \text { VS } & \% & \text { THICKNESS } \\ (\text { FEET }) & (\text { FEET }) & (\text { FT/SEC }) & (\text { FT/SEC }) & (\text { FT/SEC }) & \text { DIFF } & (\text { FEET })\end{array}$

\begin{tabular}{|c|c|c|c|c|c|c|}
\hline 0 & 5 & 621 & 621 & 0 & 0 & 5 \\
\hline 5 & 20 & 379 & 432 & 53 & 14.0 & 15 \\
\hline 20 & 60 & 654 & 625 & -29 & -5.0 & 40 \\
\hline 60 & - $\quad 95$ & 971 & 973 & 2 & 0.2 & 35 \\
\hline 95 & 115 & 1351 & 1032 & -319 & -31.0 & 20 \\
\hline 115 & 190 & 893 & 892 & -1 & -0.1 & 75 \\
\hline
\end{tabular}




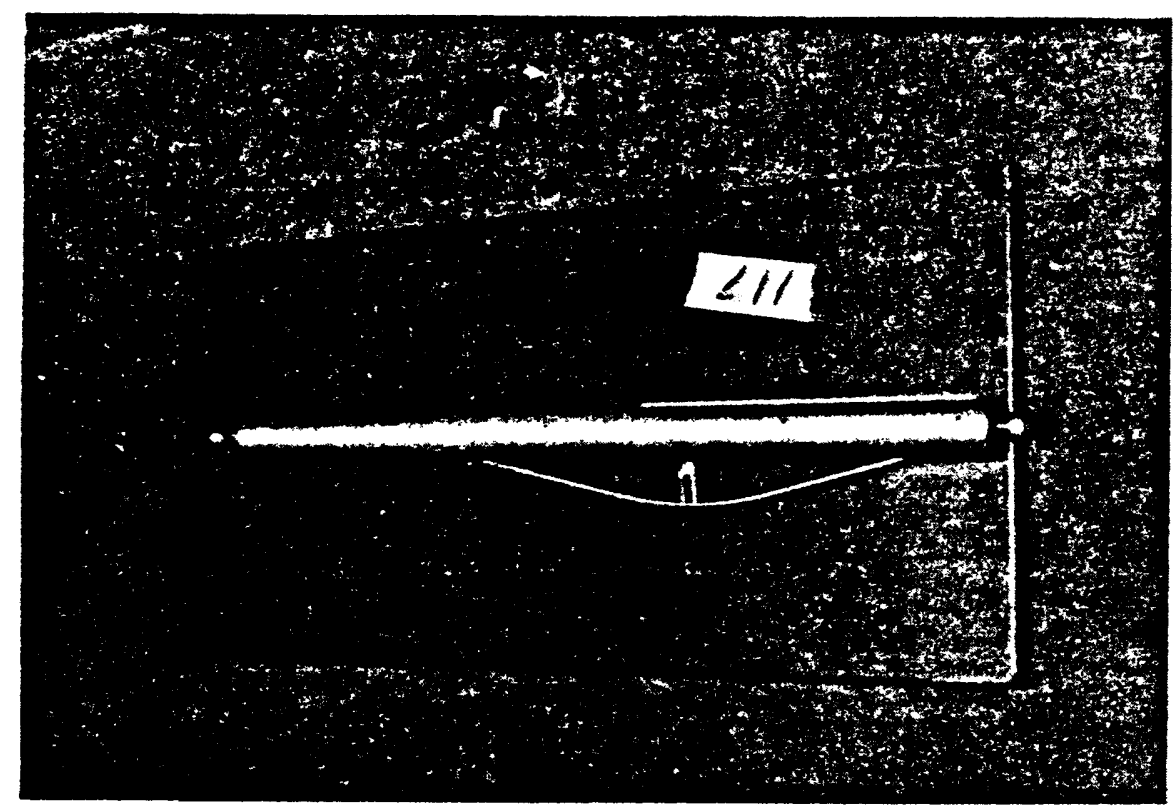

Sidewall clamping triaxial borehole geophone.

FIG. 2

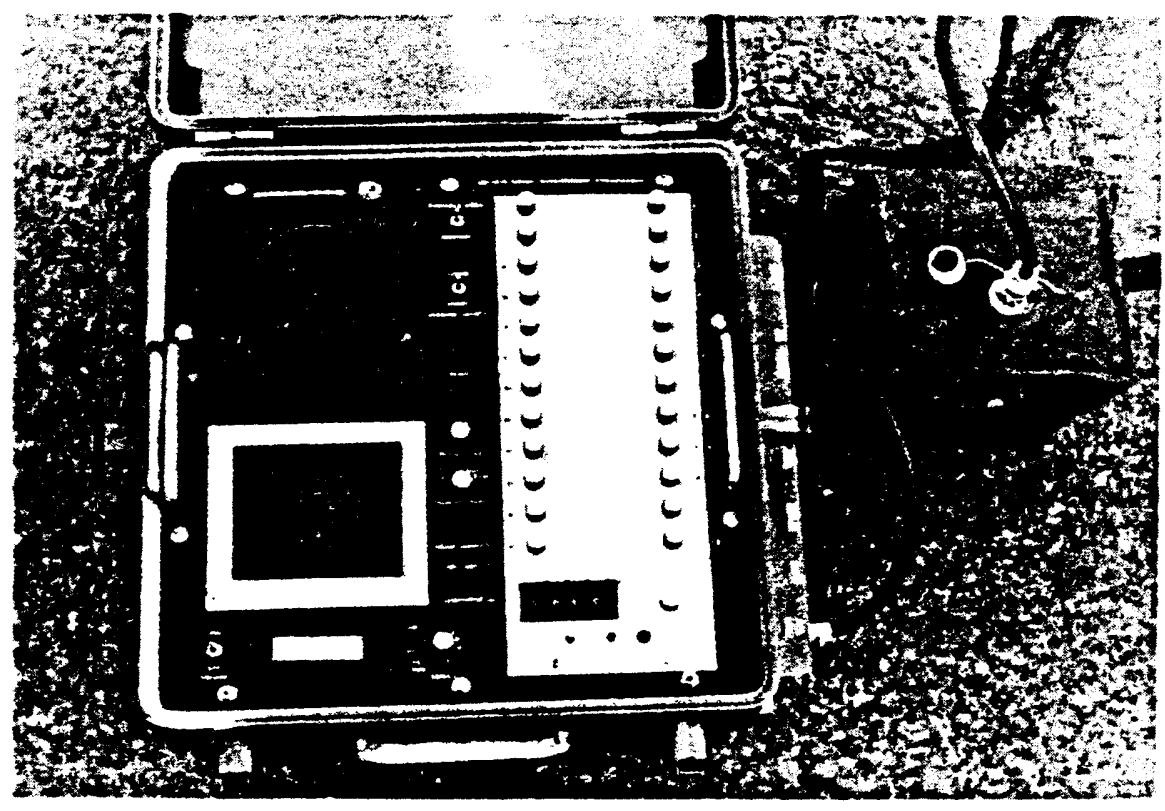

Twelve channel signal enhancement seismograph.

FIG. 3 


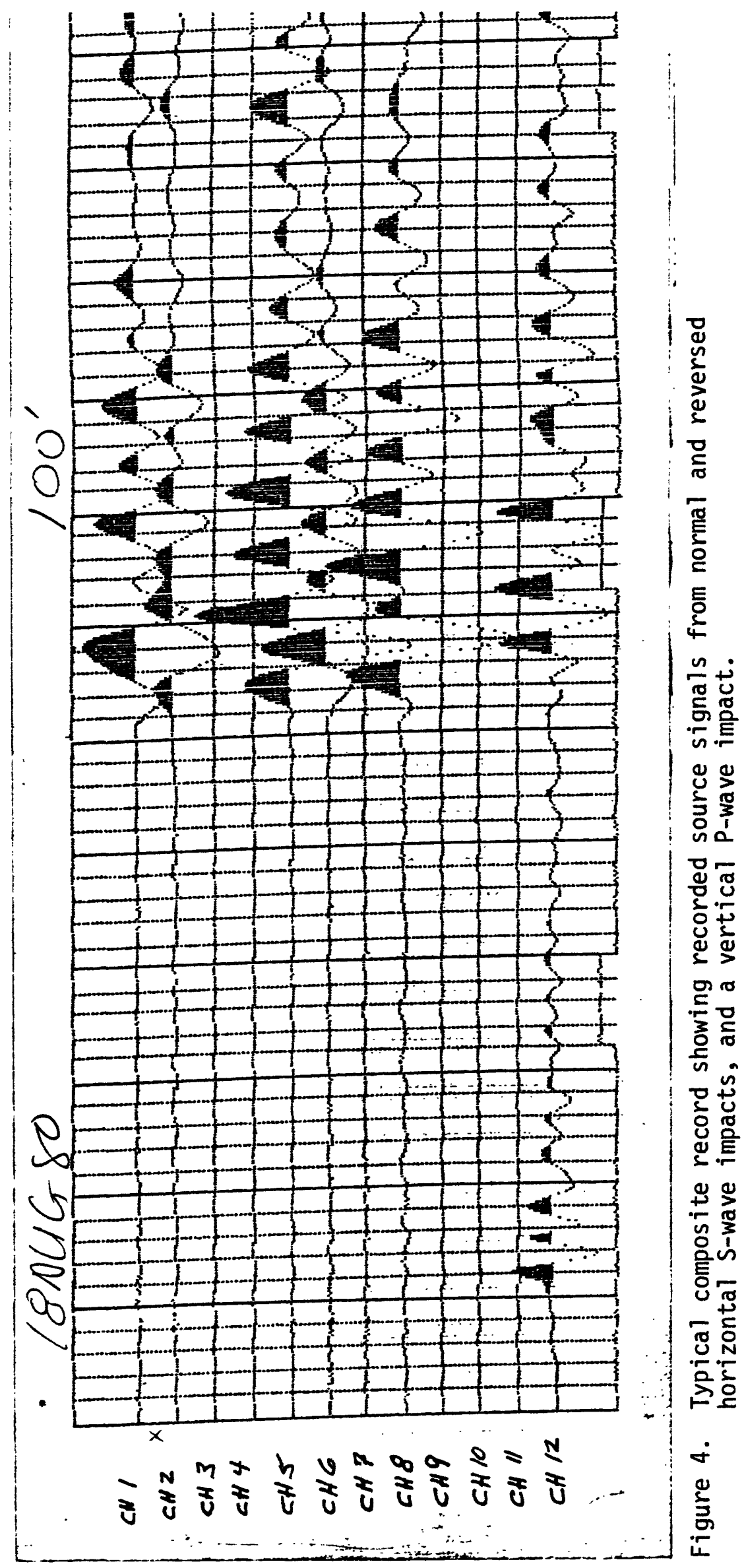



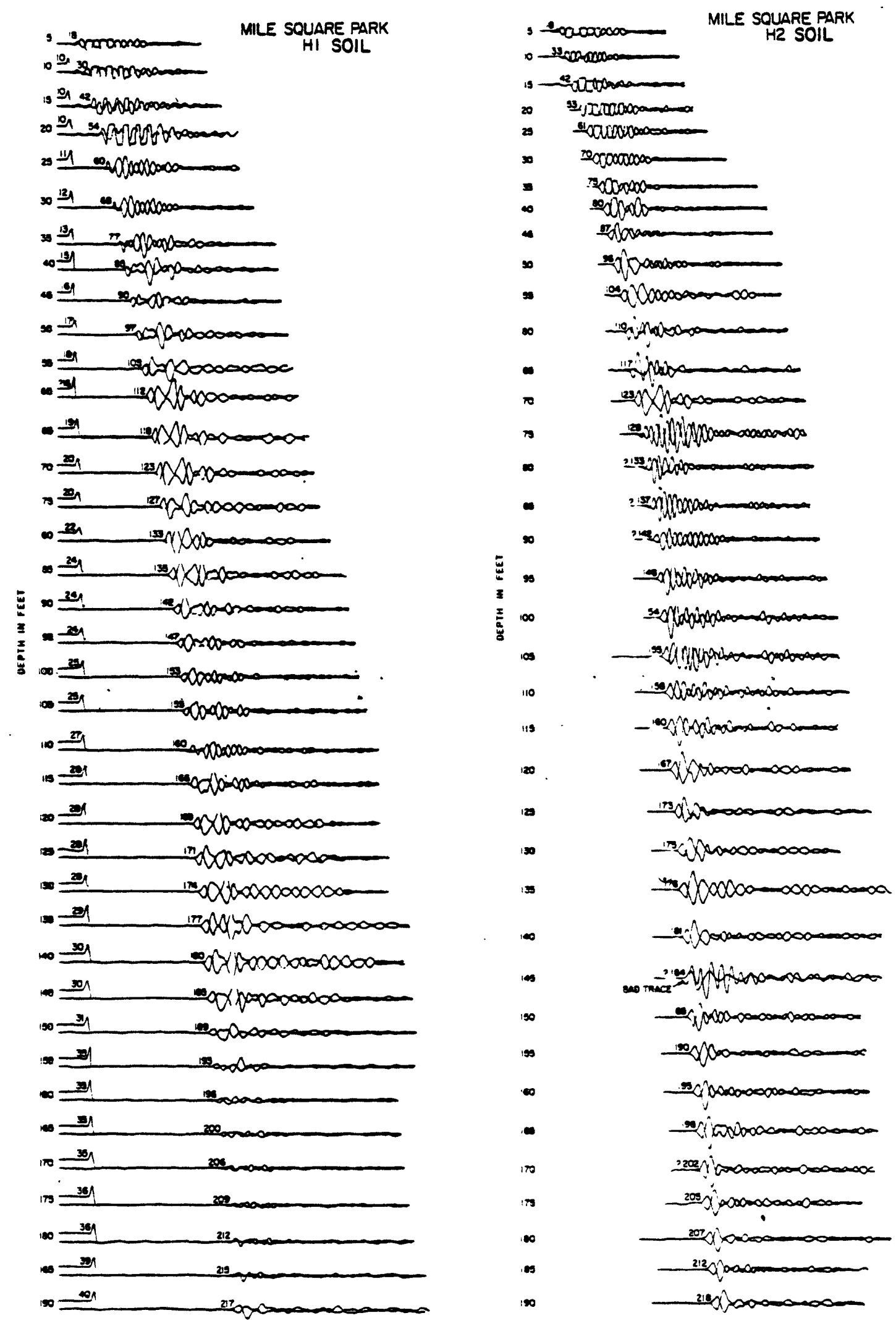

FIG. 5

Composite time histories of normal and reversed S-wave source polarity on soil at sample depths in the borehole as registered by both horizontal components. P-wave onset pulse as recorded by the vertical component is also shown. 


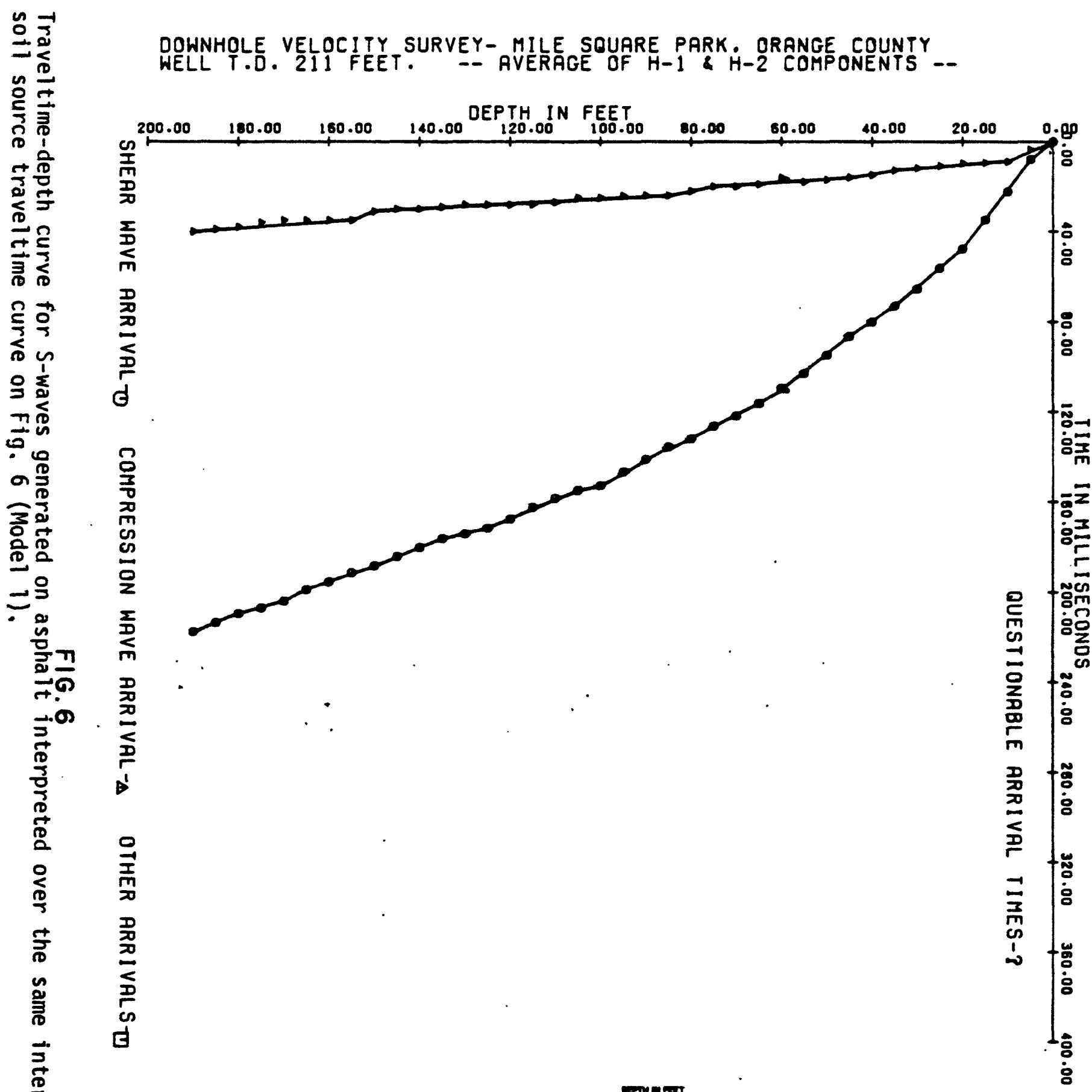

号 

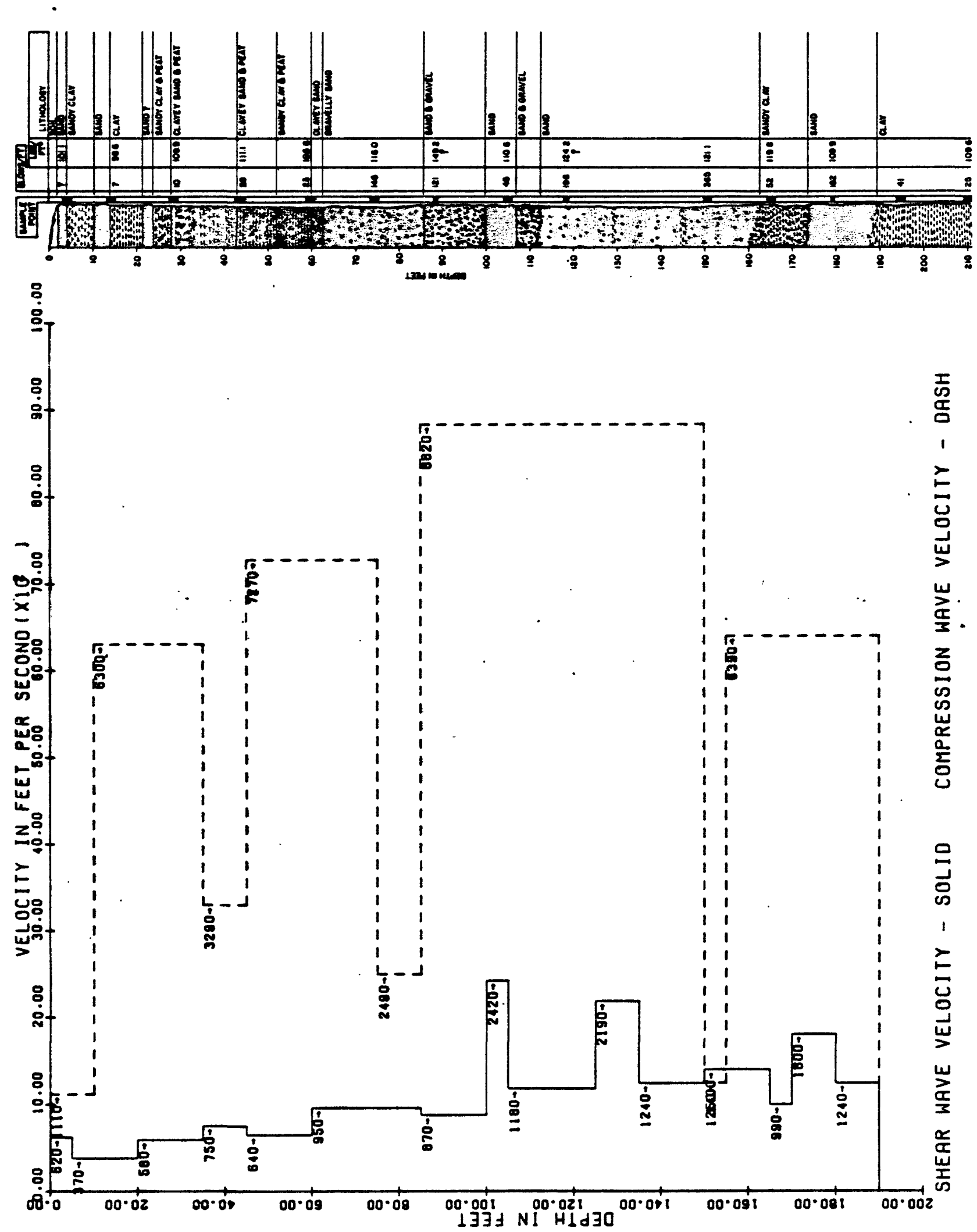

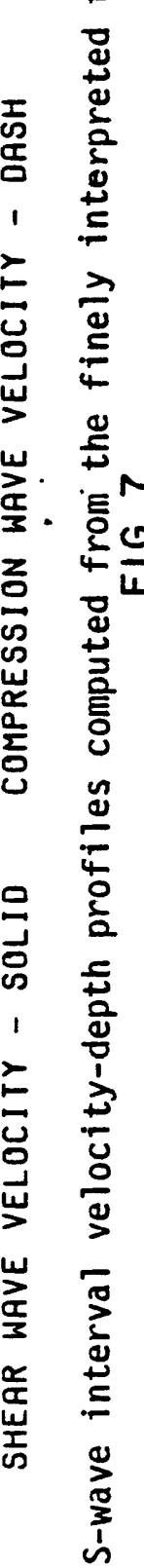

á 

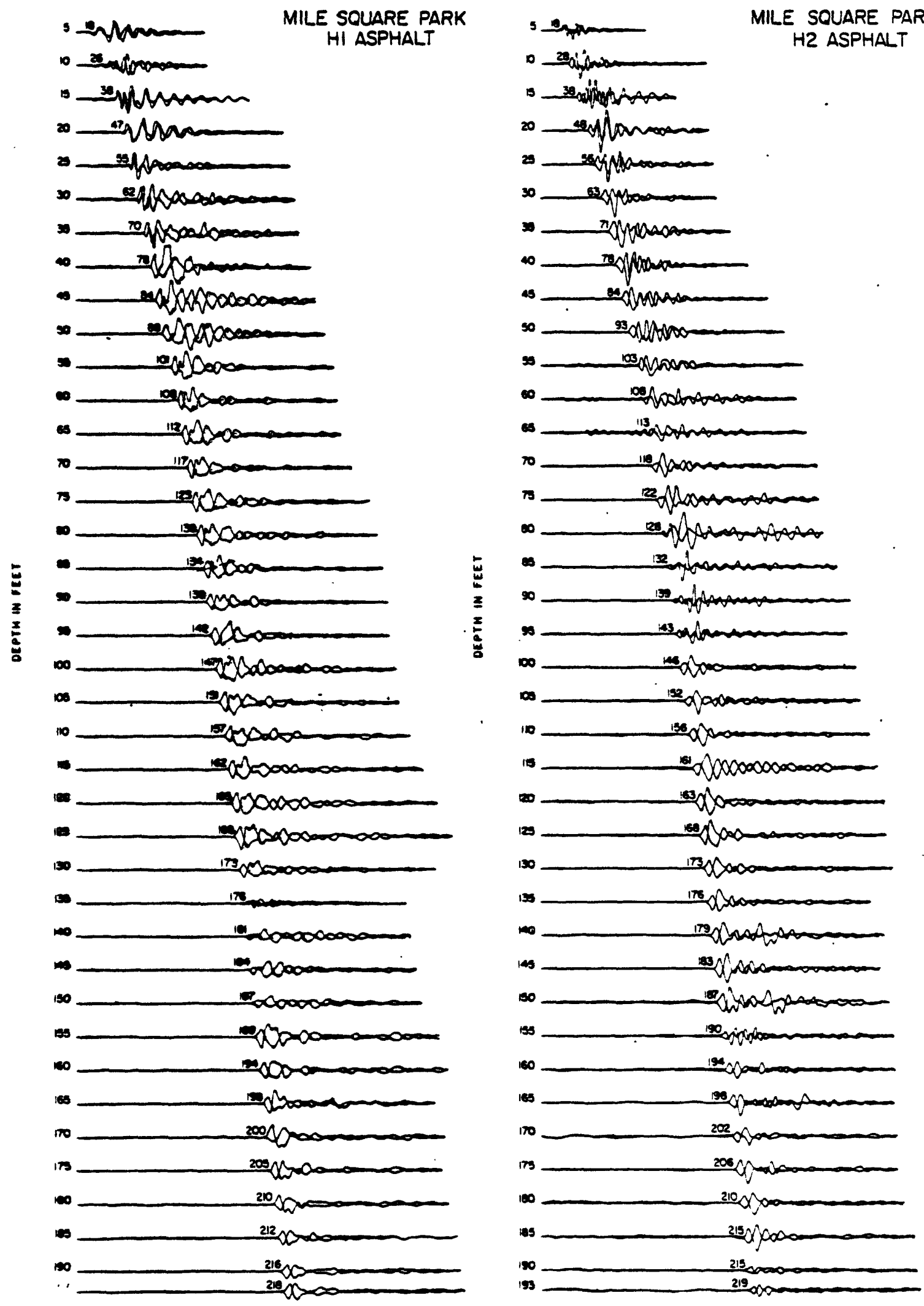

FIG. 8

Composite time histories of normal and reversed S-wave source polarity on asphalt at sample depths in the borehole as registered by both horizontal components. 


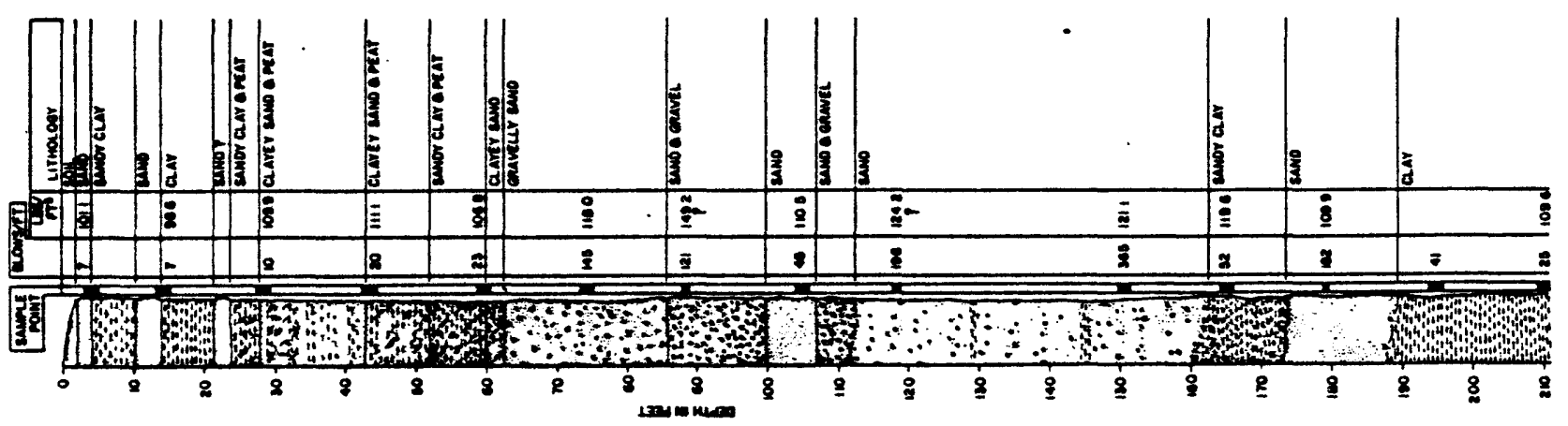

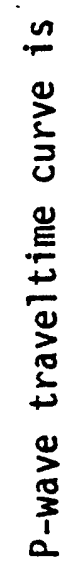

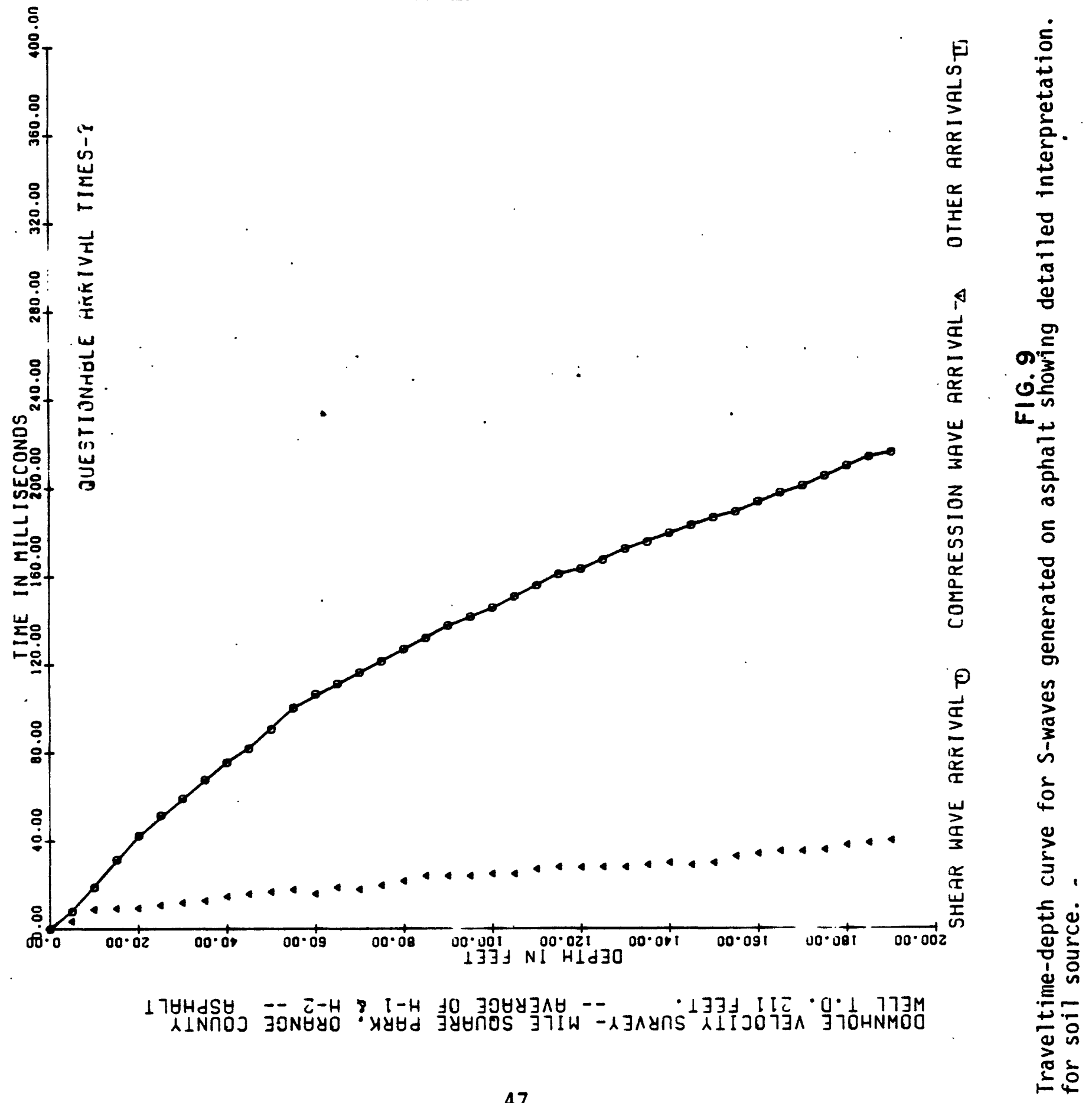




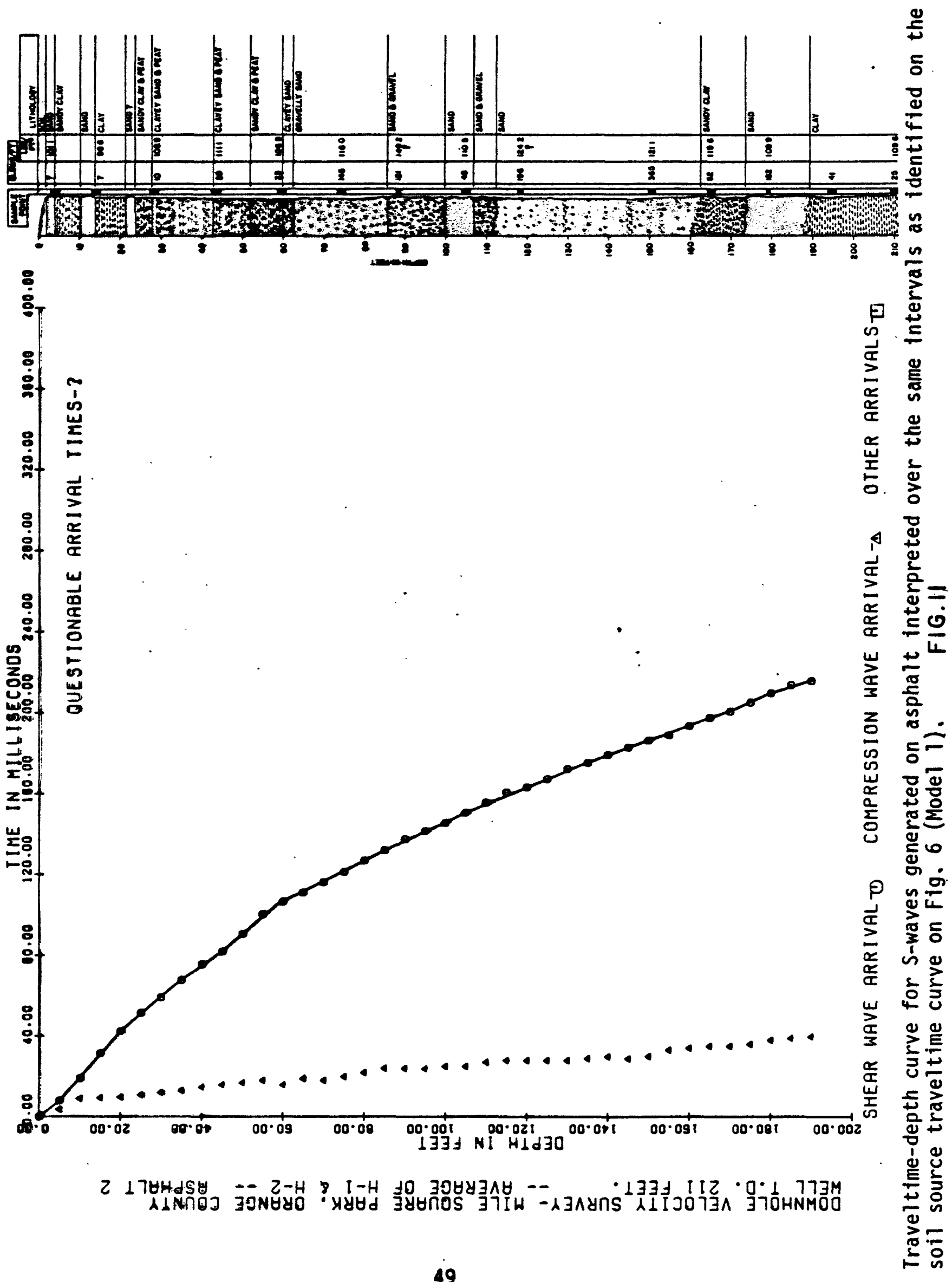



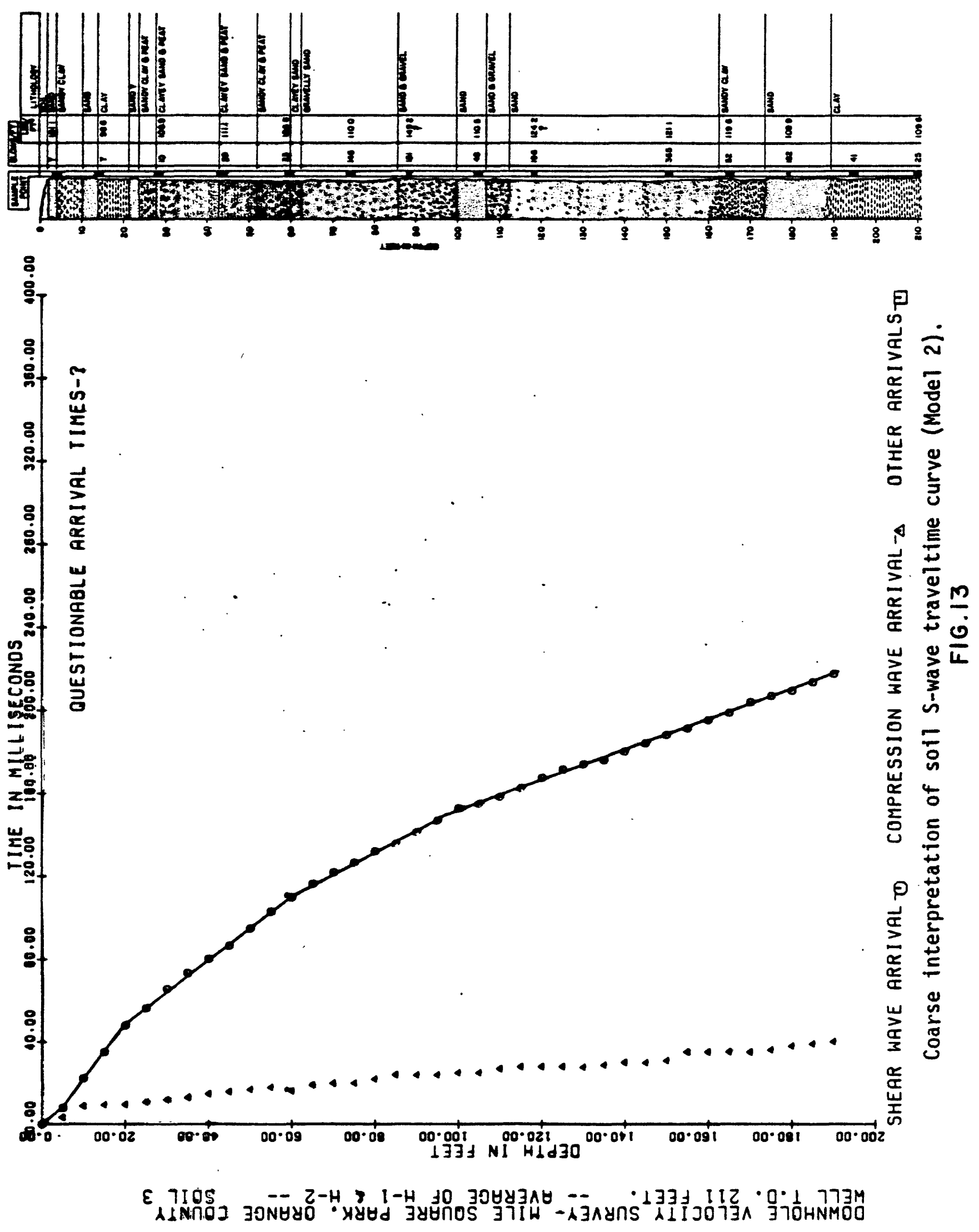


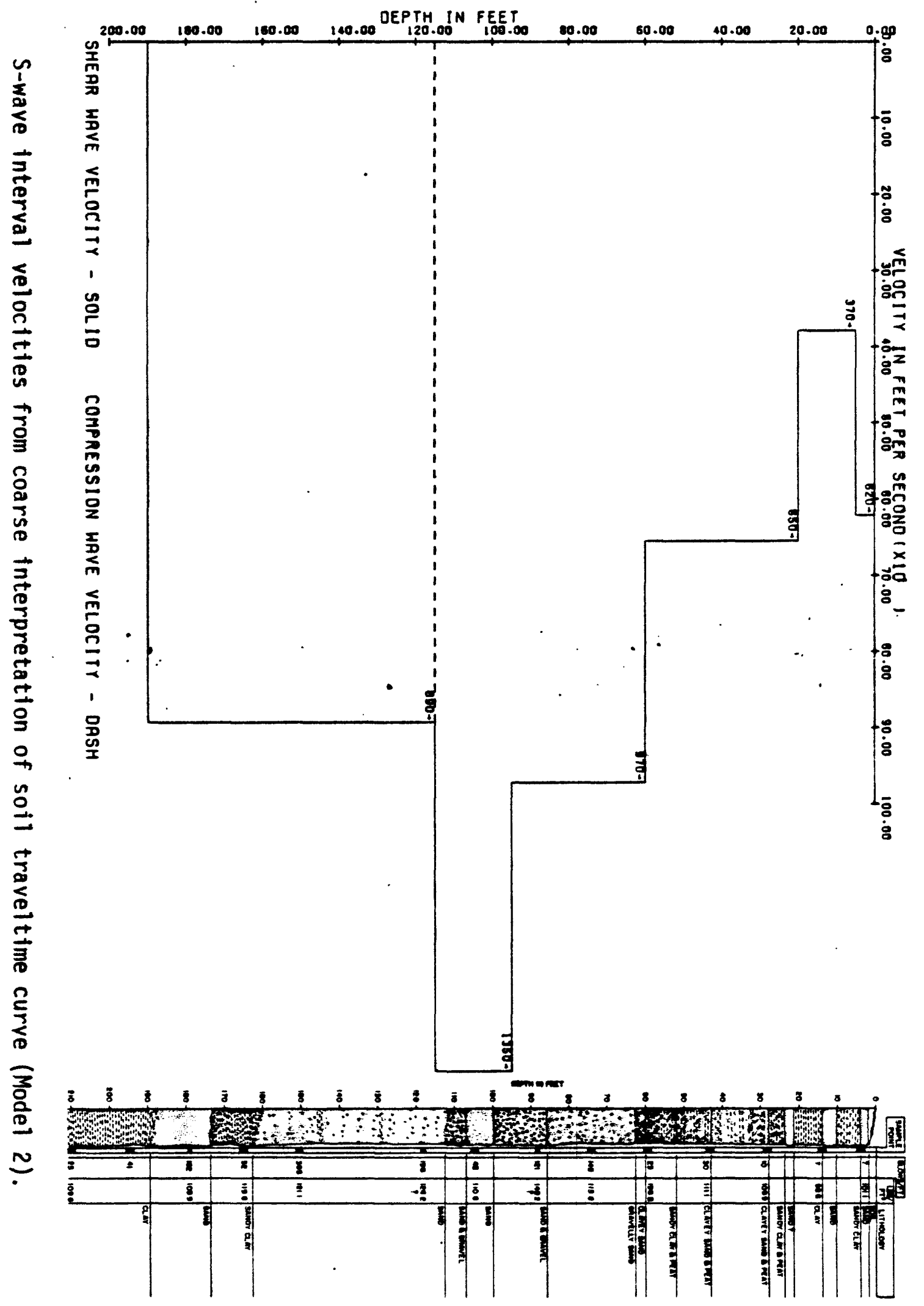



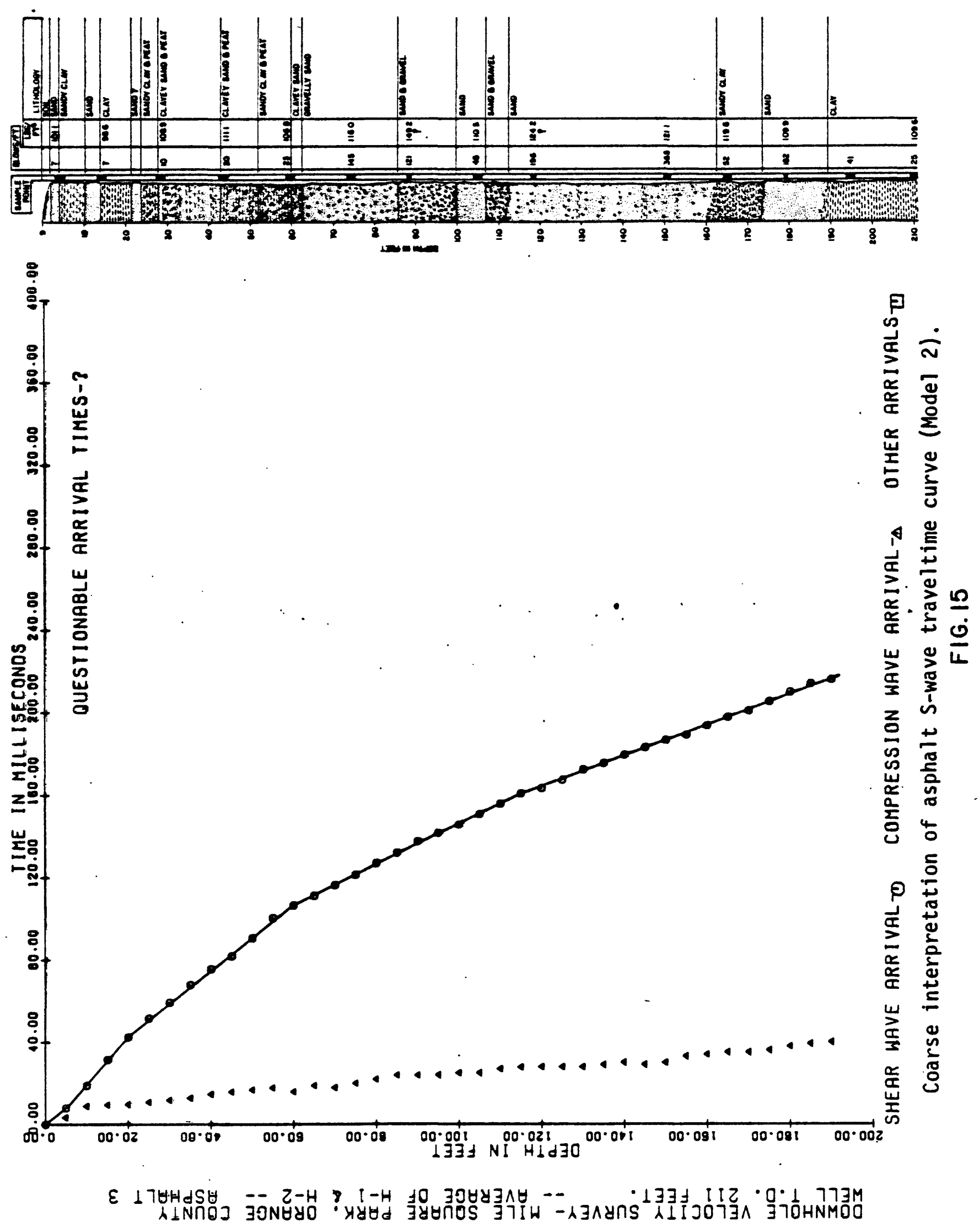


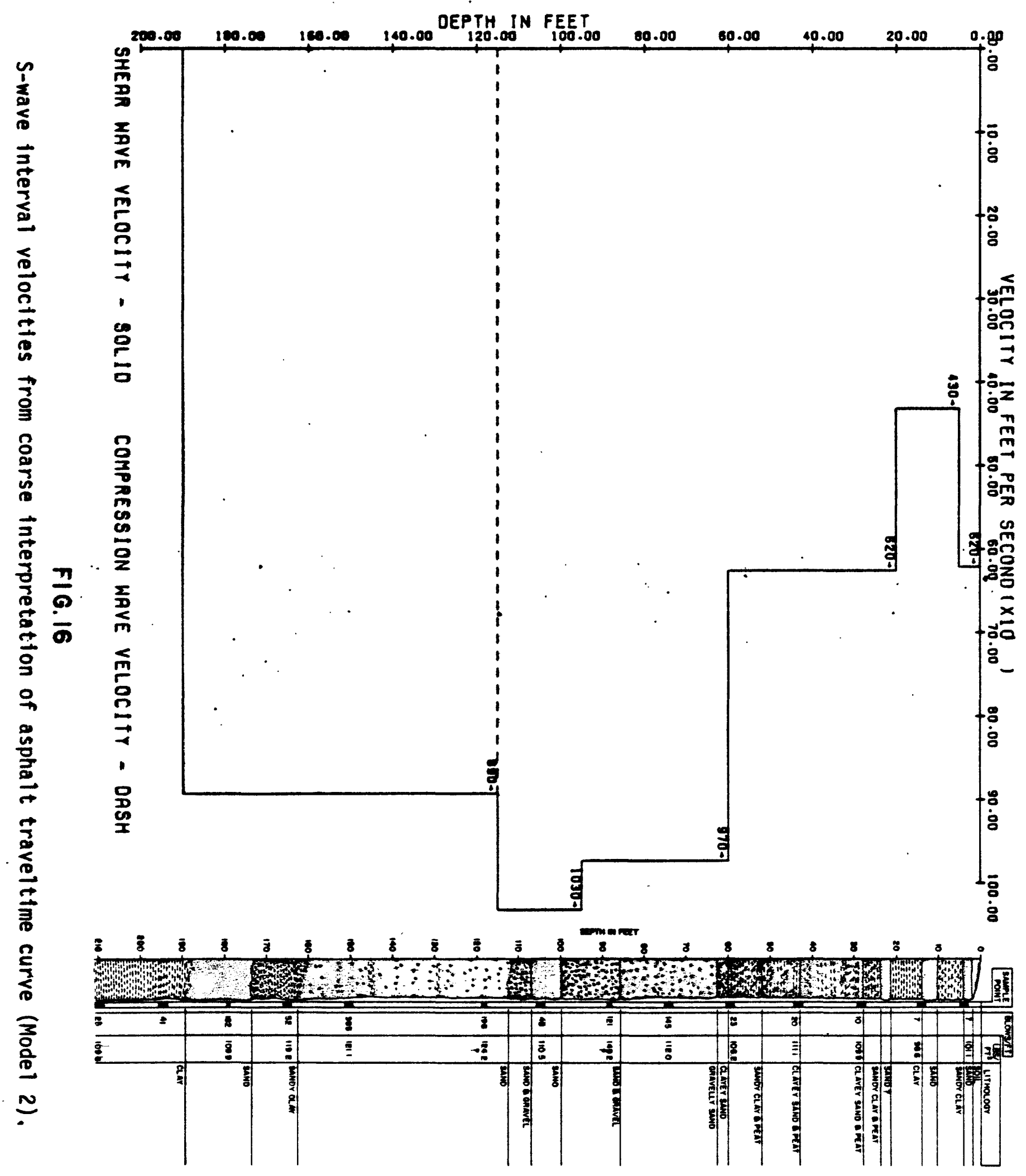


Figure 17. Raypath for downhole velocity survey with source plank on asphalt.

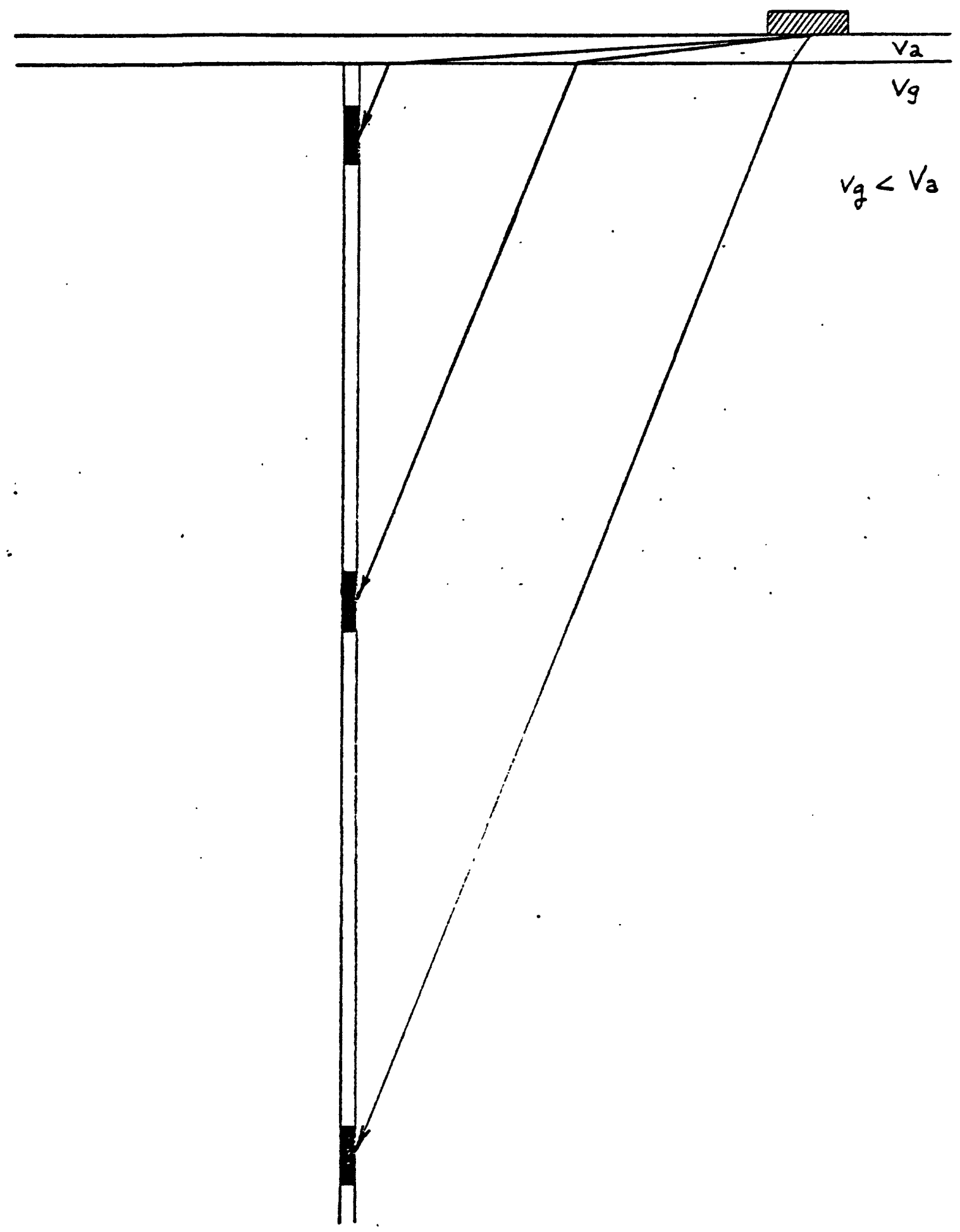




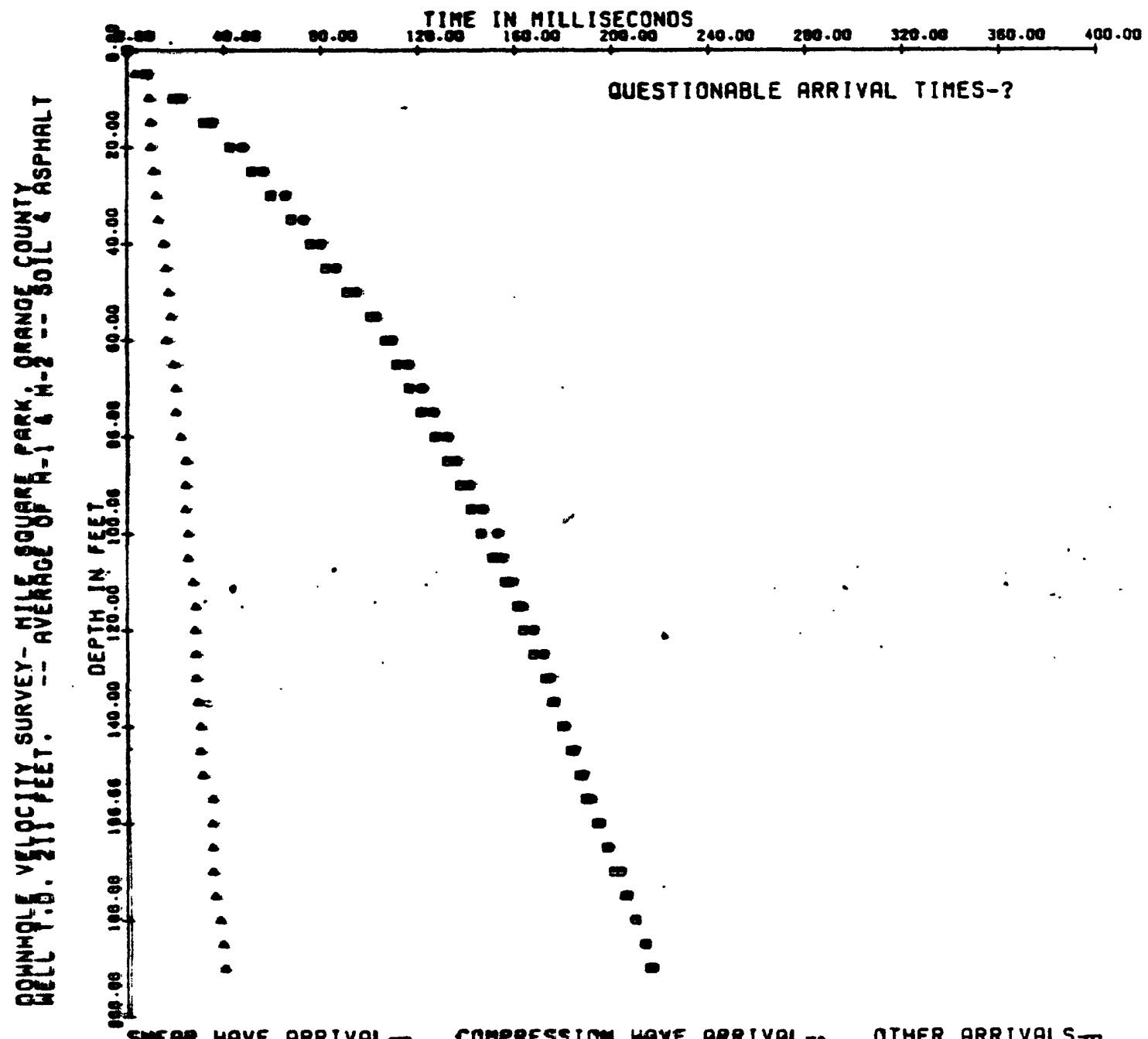

COMPRESSION HAVE RRRIVAL-A OTHER ARRIVALST

Comparison of traveltime curves for S-waves generated on soil and asphalt. FIG.I8 
APPENDIX A

INPUT ARRIVAL TIMES, CORRECTED TRAVELTIMES, AND COMPUTED INTERVAL VELOCITIES 
SOIL - FINE INTERPRETATION MODEL I FIGURES 6 AND 7 

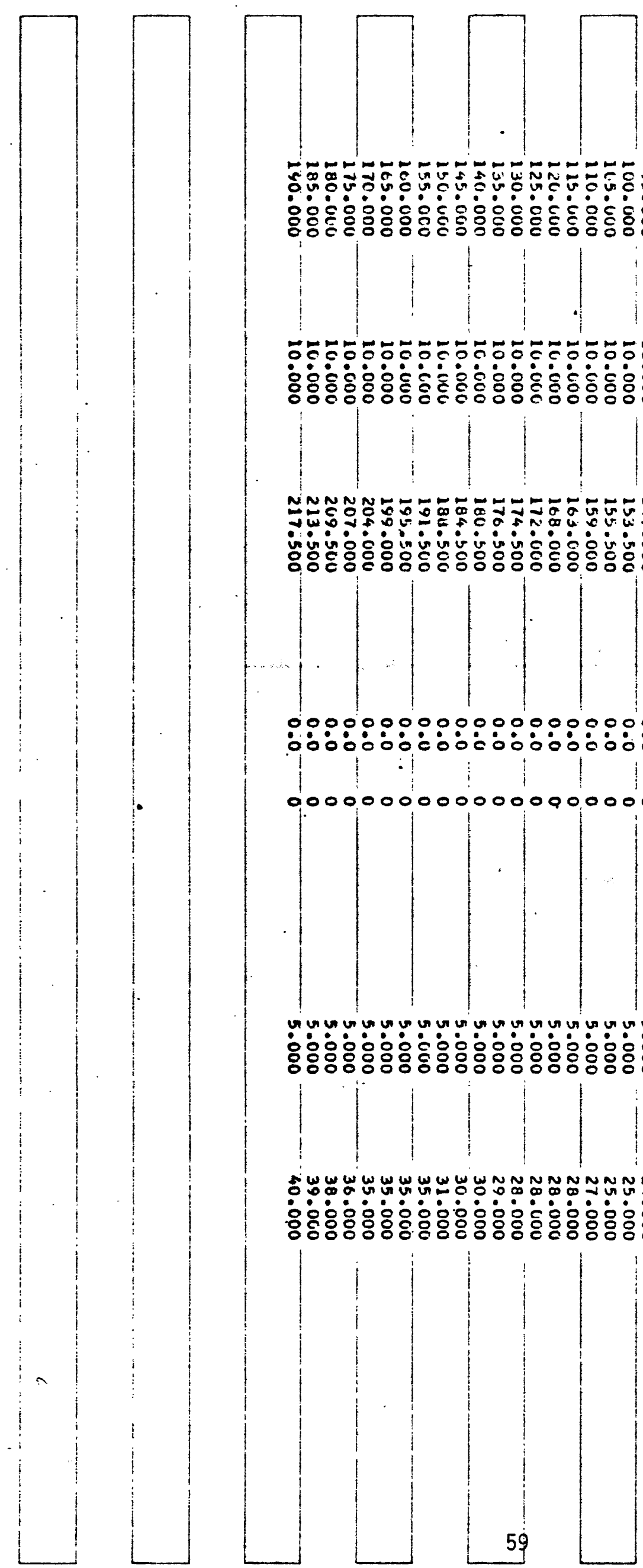

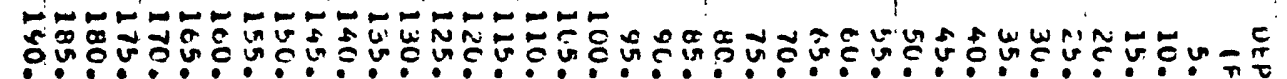

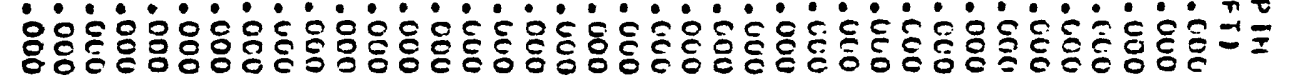

号吉

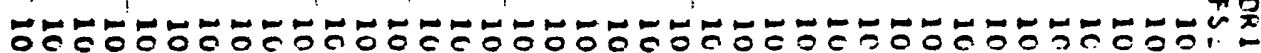

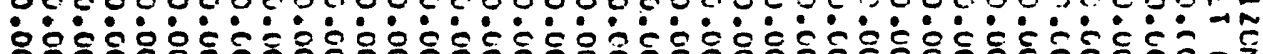

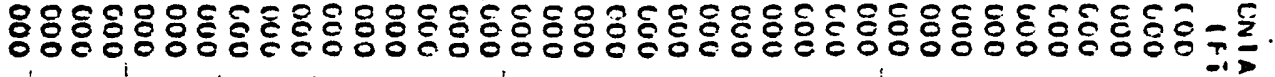
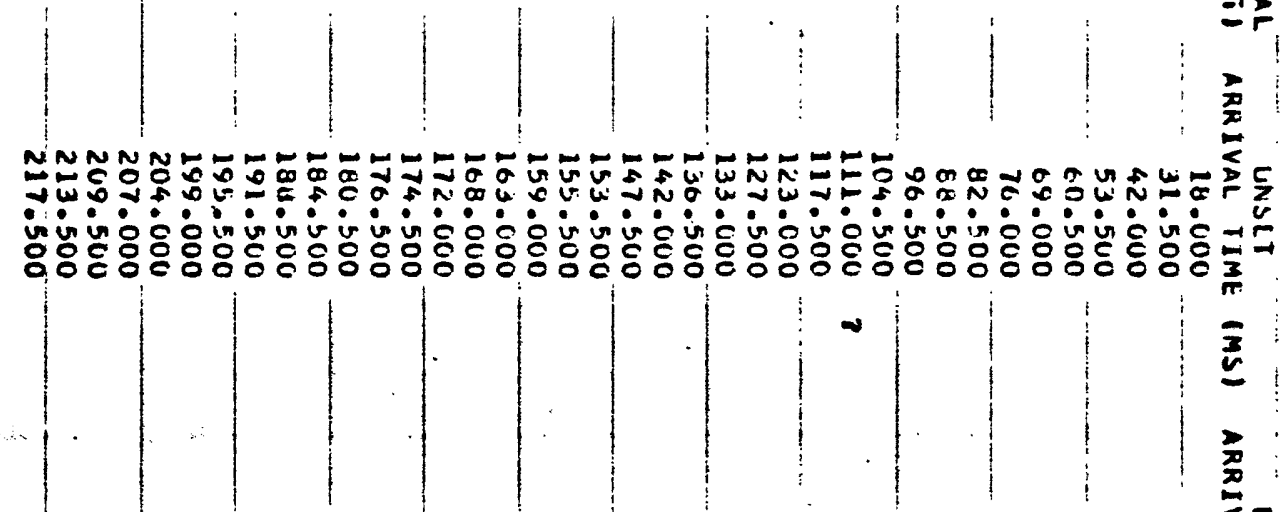

000000000000000000000000000000000000005

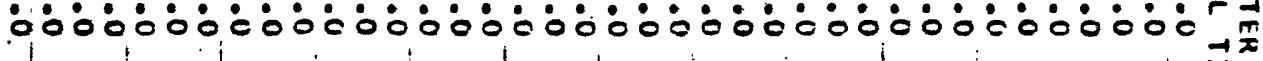
00000000000000000000000000000000000000
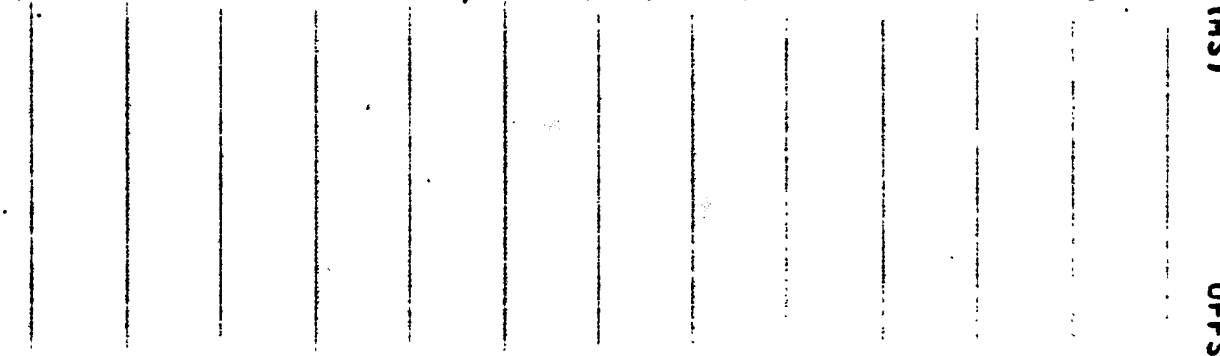

$\underline{\underline{3}}$

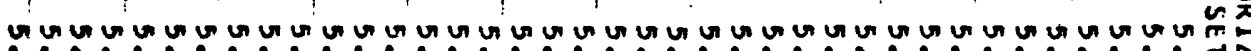

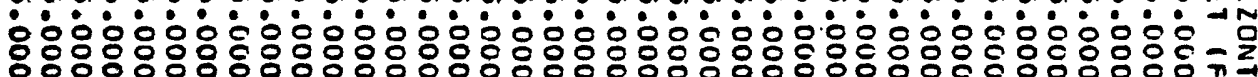

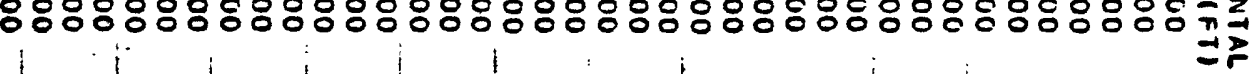

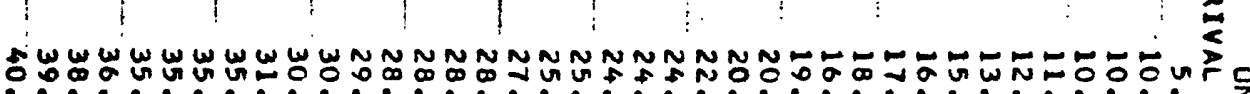

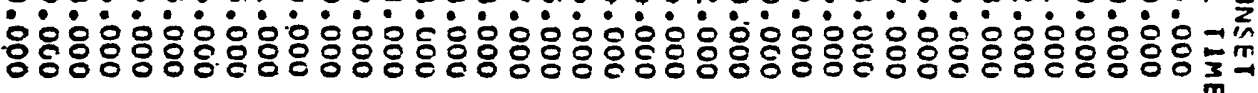




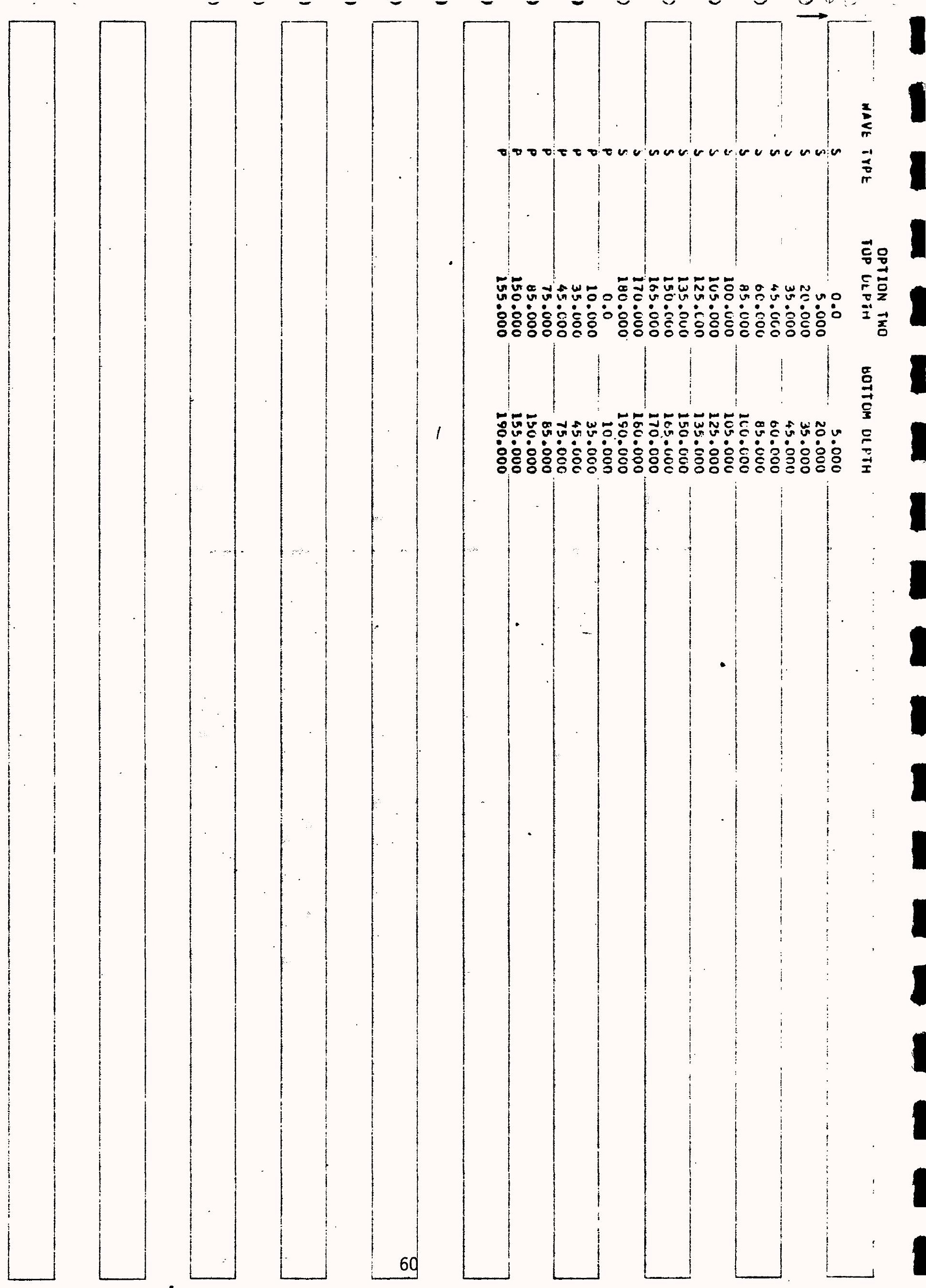





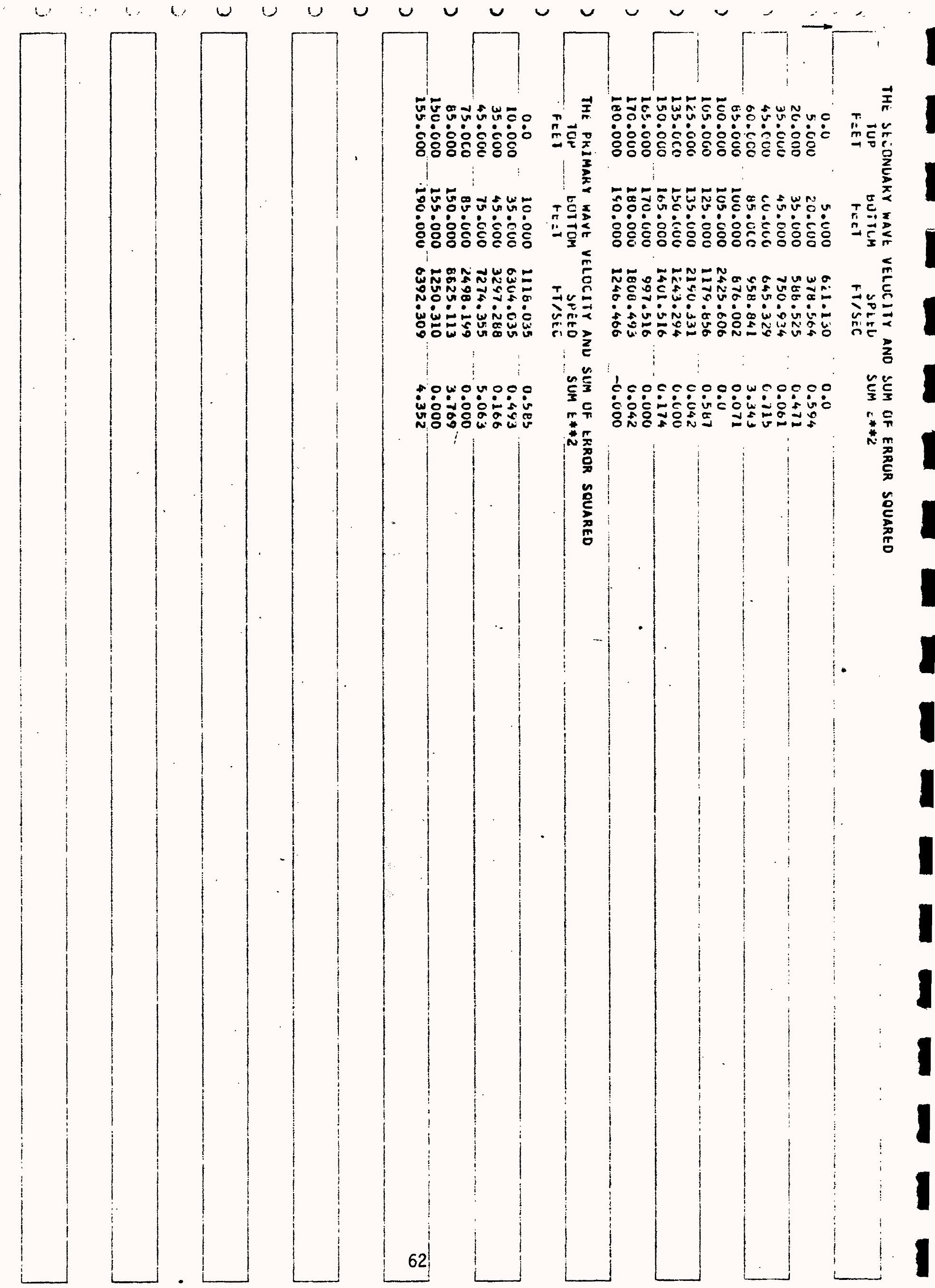




\section{ASPHALT - FINE INTERPRETATION}

FIGURES 9 AND 10 : 
$\partial \rightarrow 0$
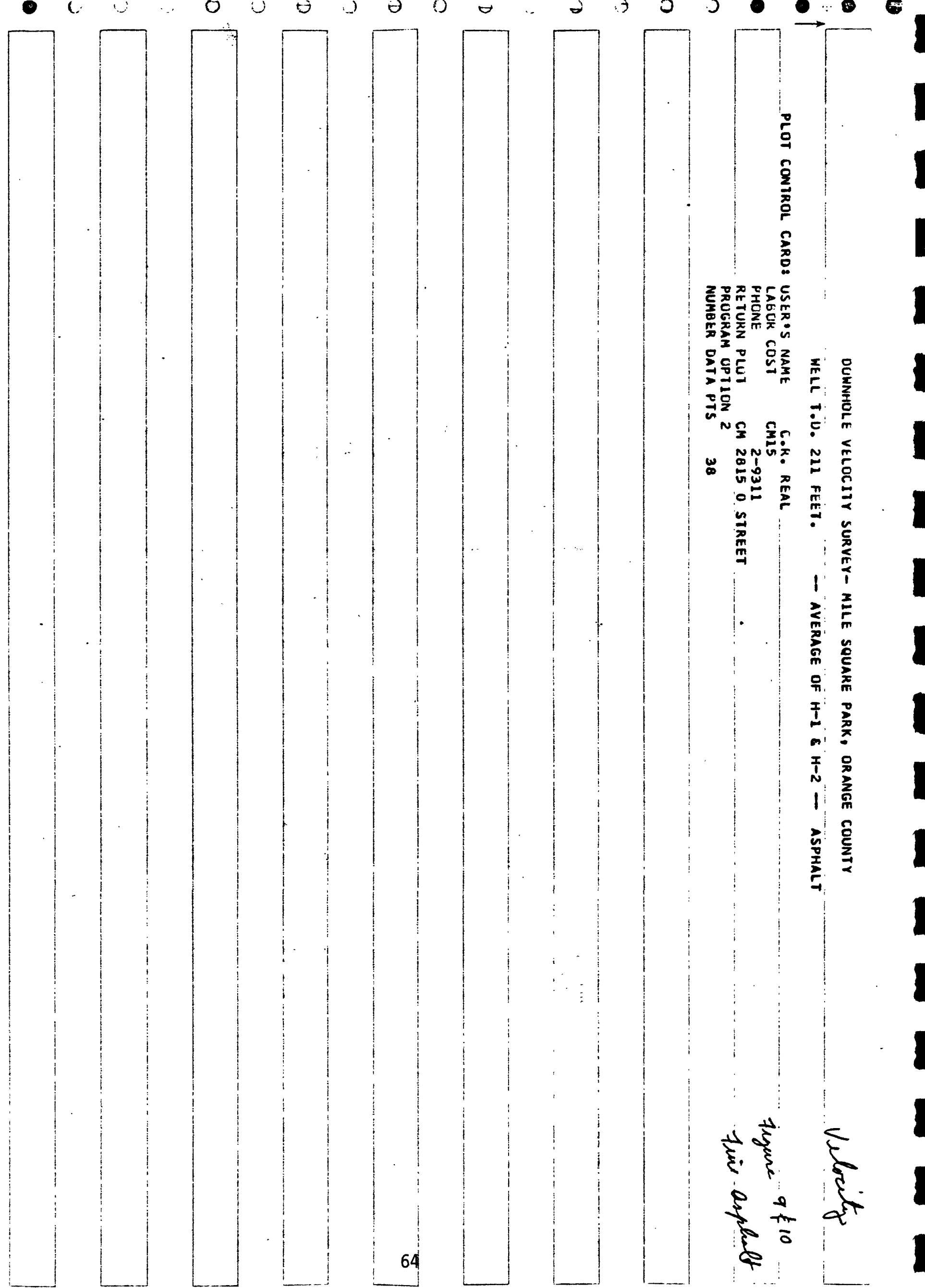


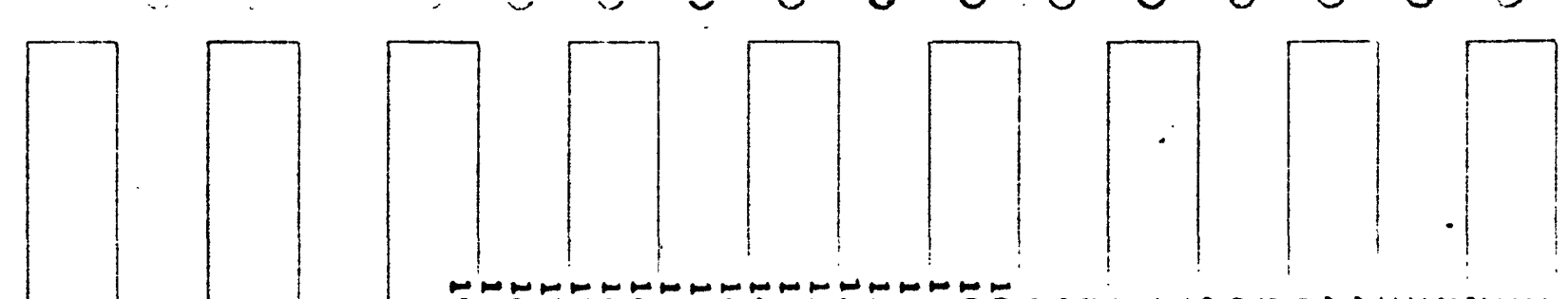

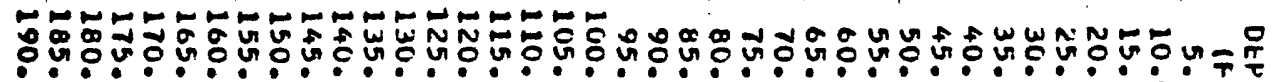

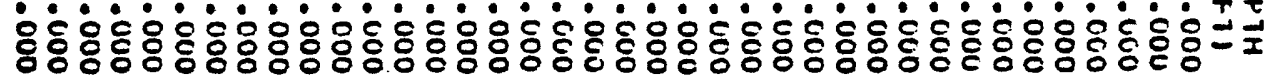

号昰

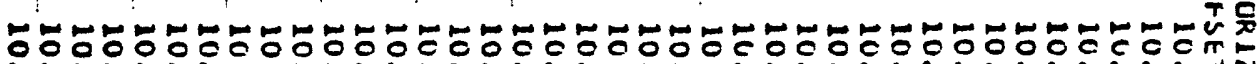

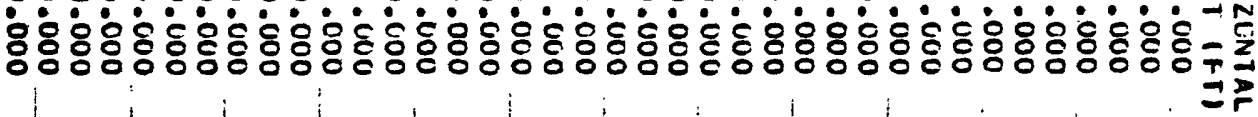
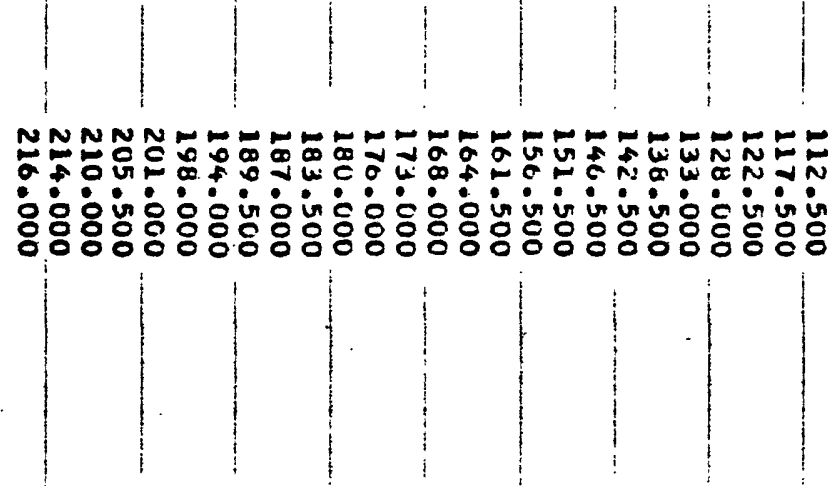

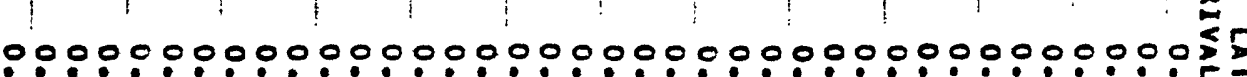
ío:

00000000000000000000000000000000000000

要

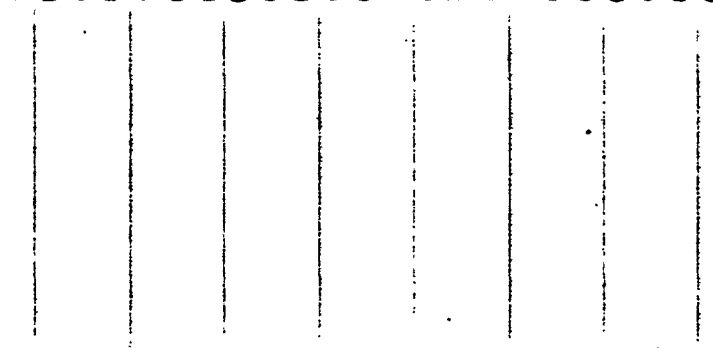

竞

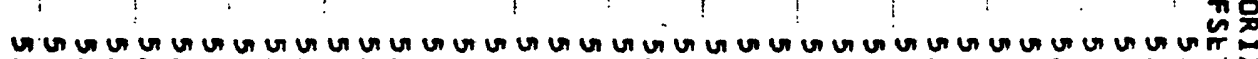

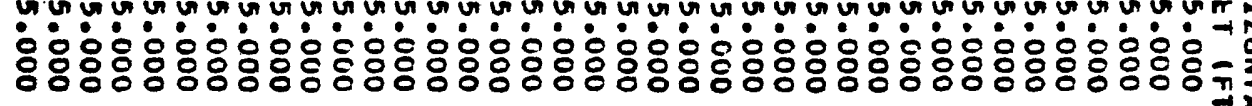

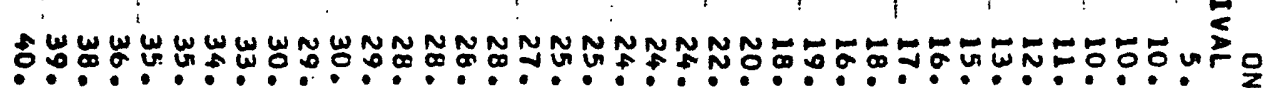

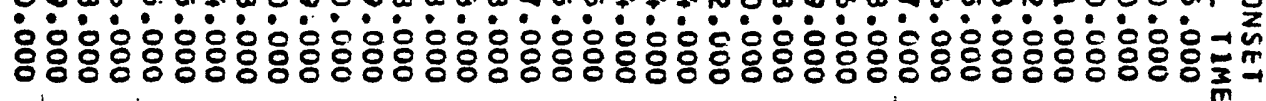




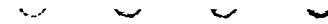



$\begin{array}{lllllllllllllll}0 & 0 & 0 & 0 & 0 & 0 & 0 & 0 & 0 & 0 & 0 & 0 & u & u & 0\end{array}$

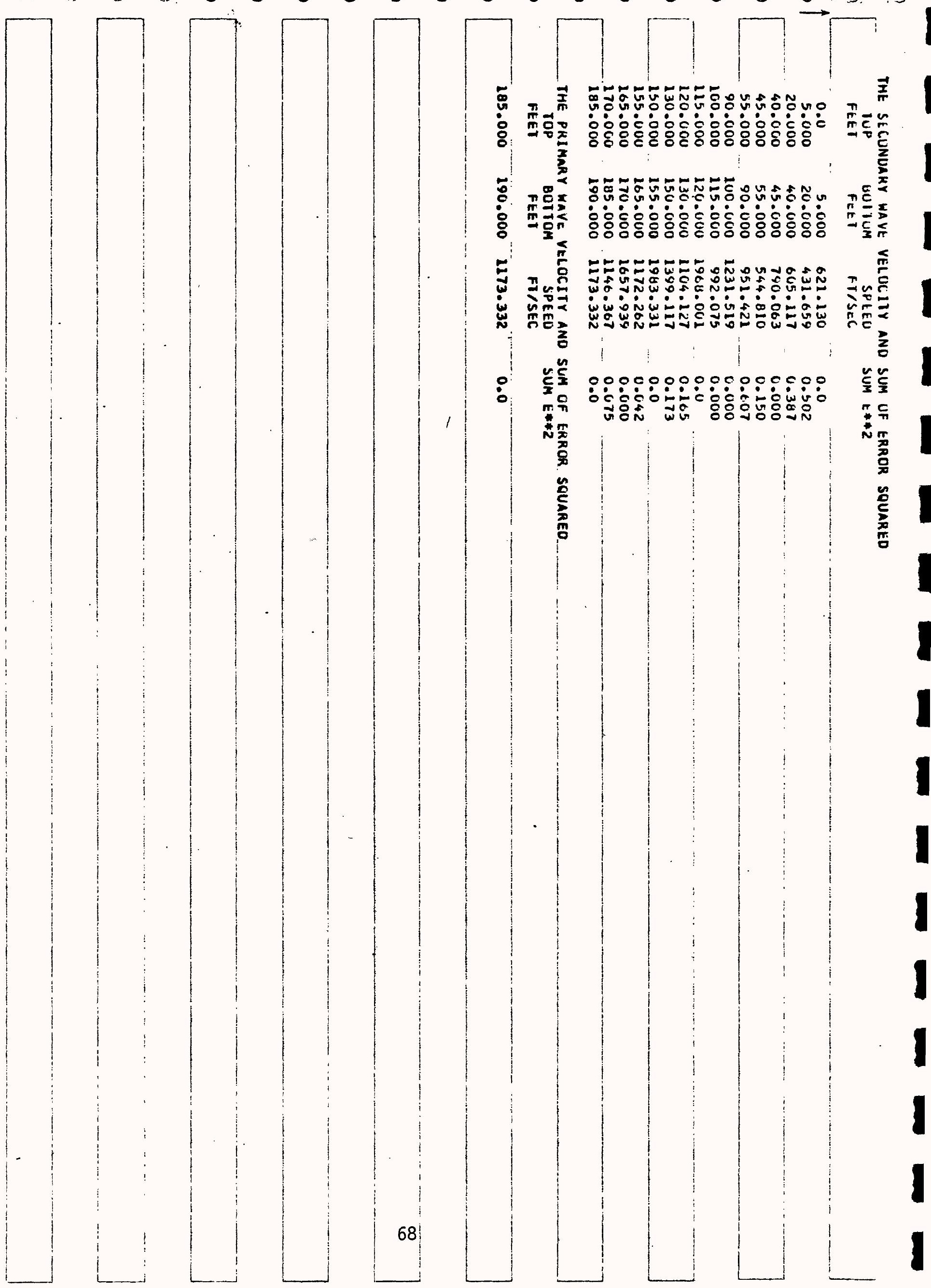


ASPHALT - FINE INTERPRETATION MODEL I

- FIGURES 11' AND 12 


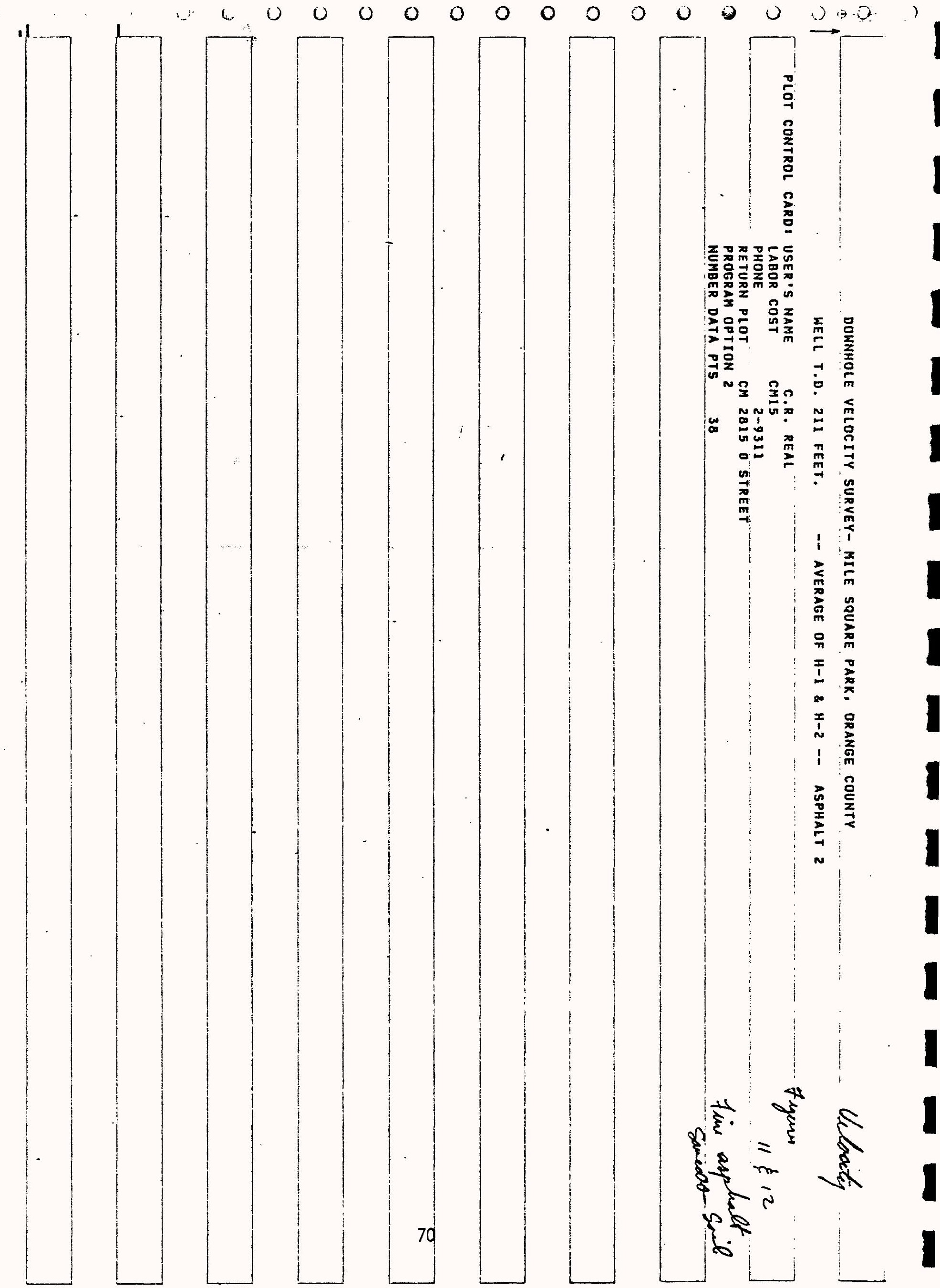




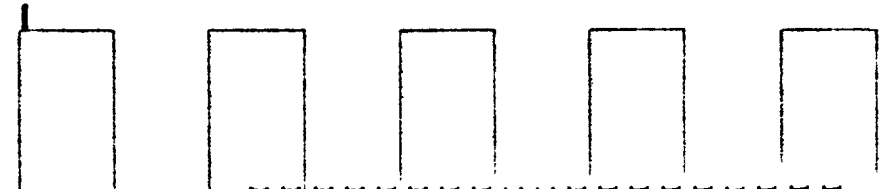

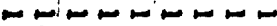

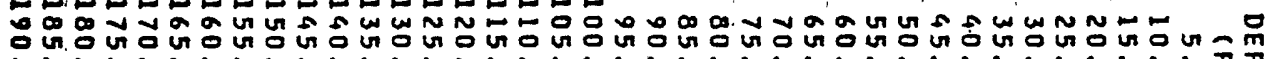
- ó ó ó ó ó

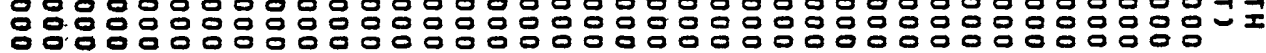

节它

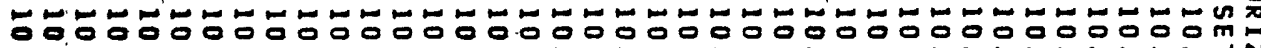

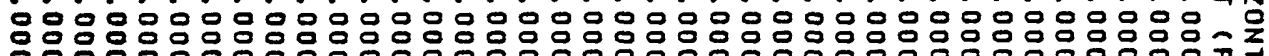

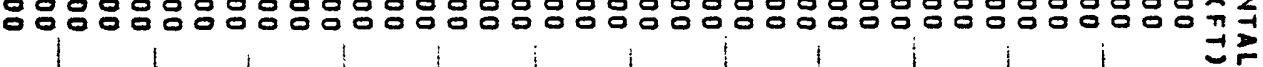

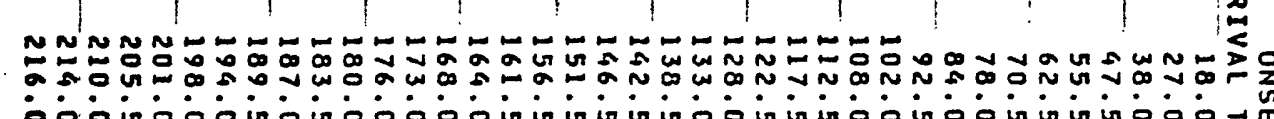

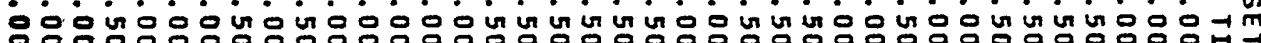

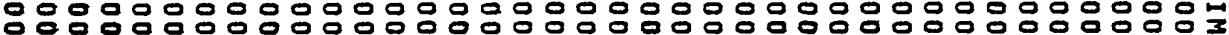
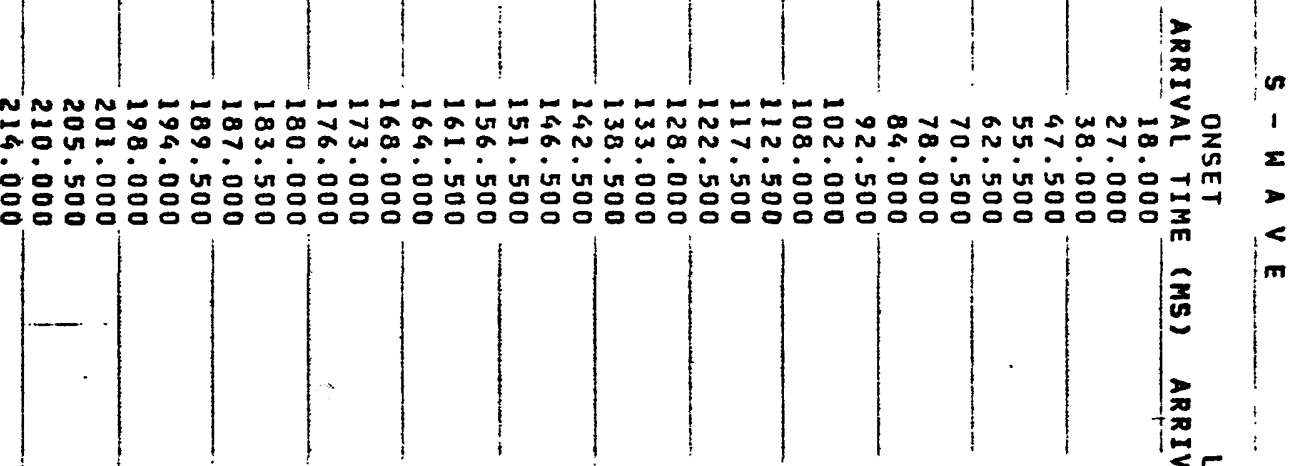

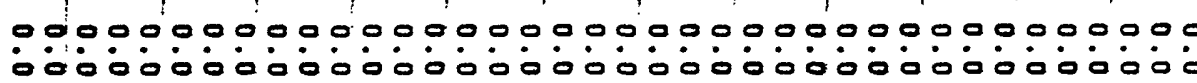

00000000000000000000000000000000000000

$\frac{3}{\mathbf{z}}$

m 
$\begin{array}{lllllllllll}0 & 0 & 0 & 0 & 0 & 0 & 0 & 0 & 0 & 0 & 0\end{array}$

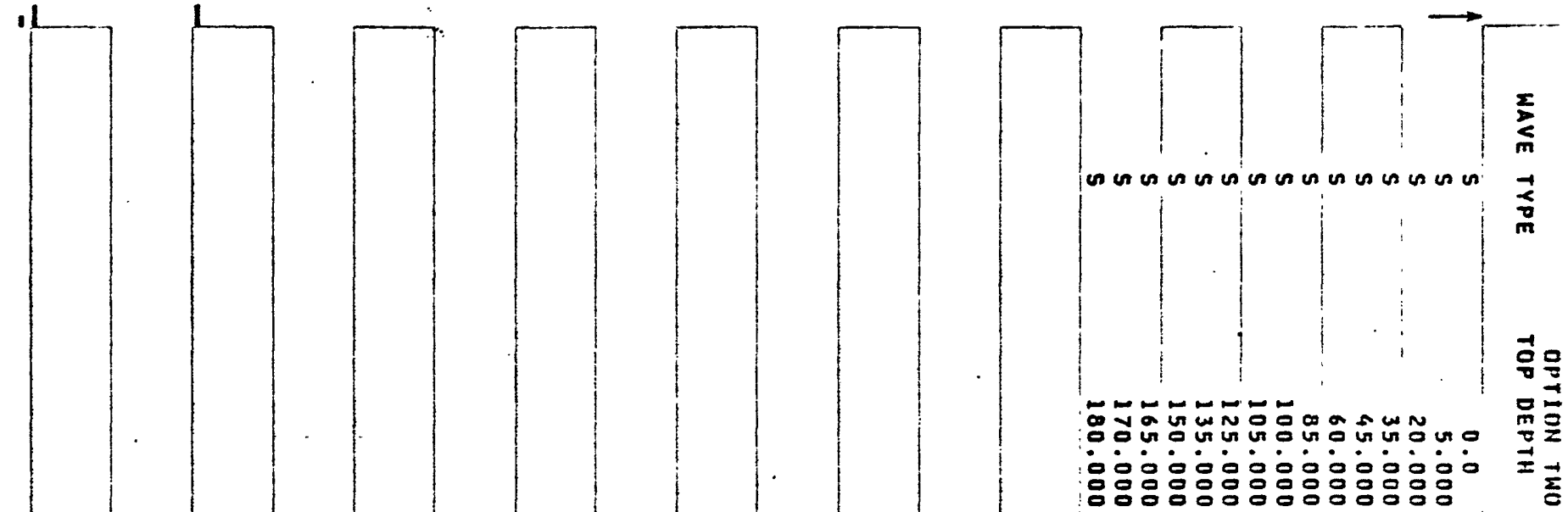

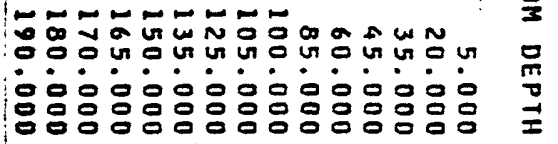

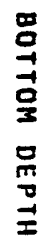



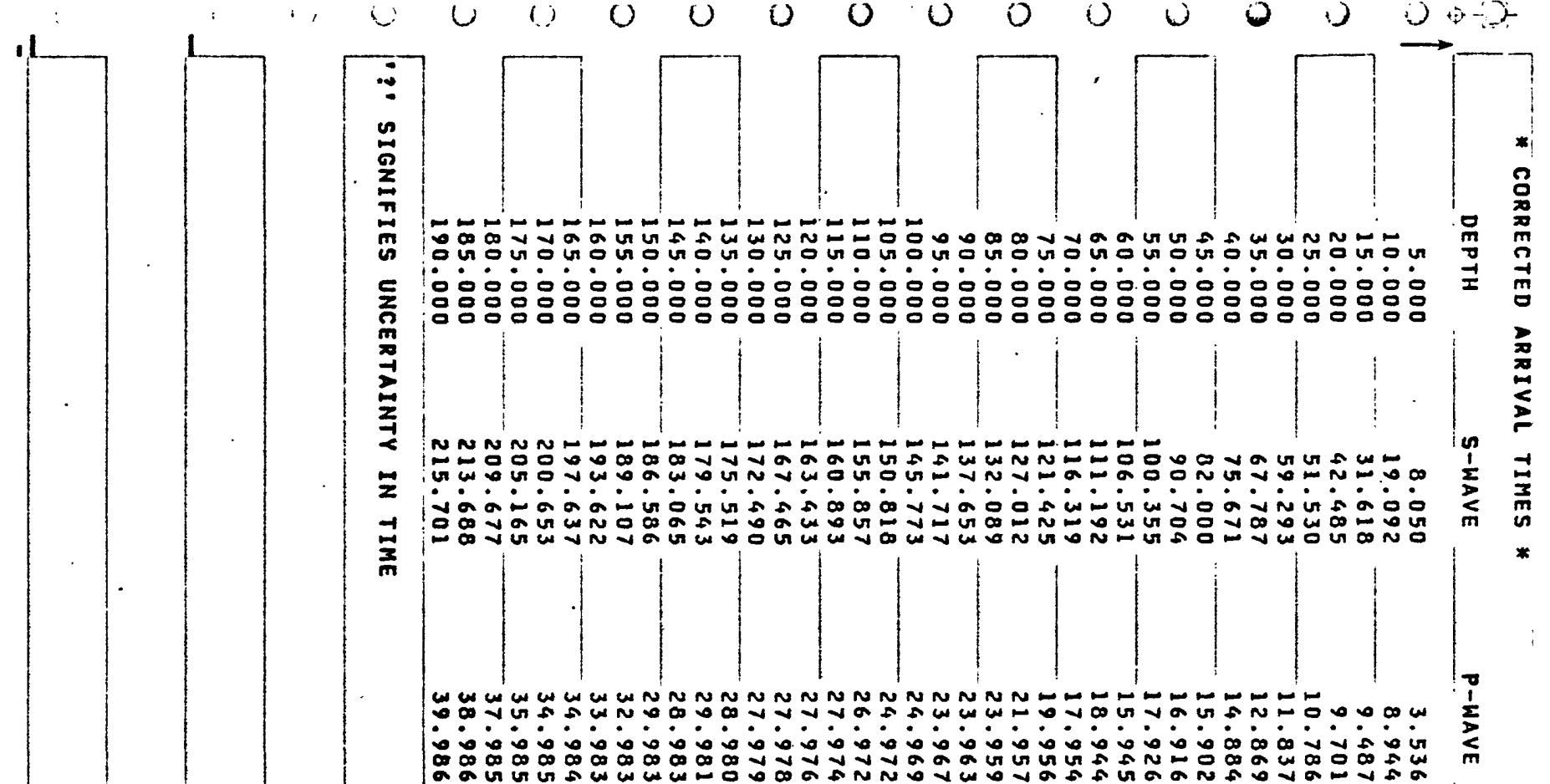

旁

00000000000000000000000000000000000000

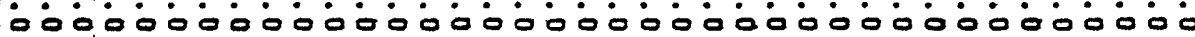




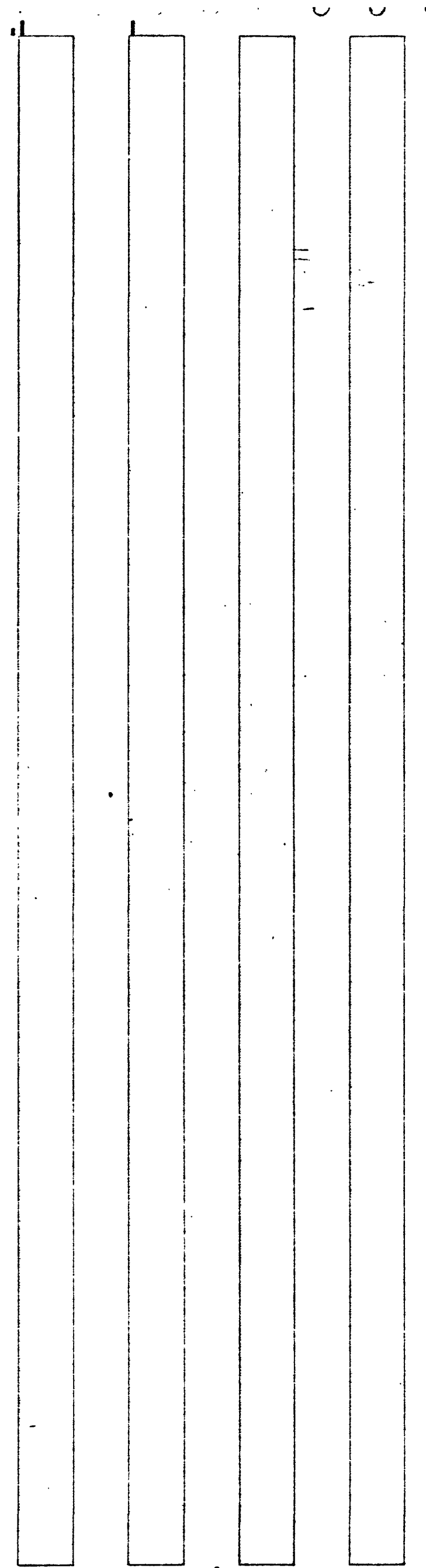

$\checkmark v$

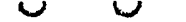

$U$

$\omega$

$\therefore$

- $\quad$ I

- m. m

| |

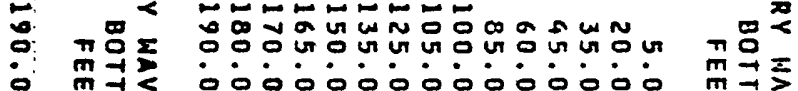

省炛

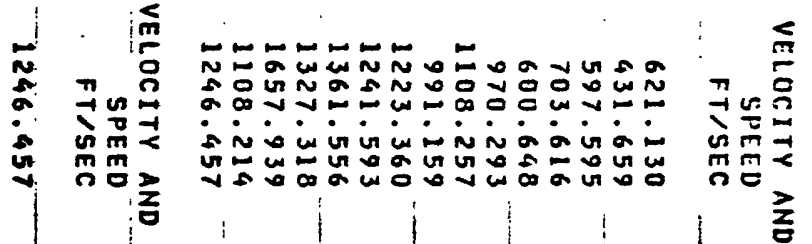

!)

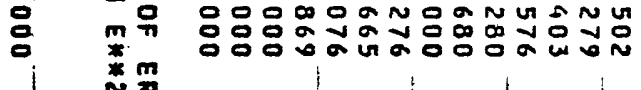

m운

学

$\left.\right|_{\substack{0 \\ 0}} ^{n}$

m

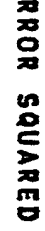


SOIL- COARSE INTERPRETATION MODEL II

FIGURES 13 AND 14 

$\begin{array}{lllllllllllll}0 & 0 & 0 & 0 & 0 & 0 & 0 & 0 & 0 & 0 & 0 & 0 & 1\end{array}$

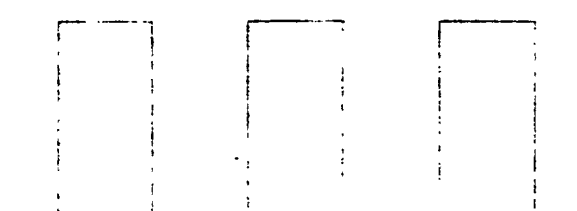

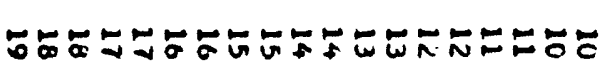

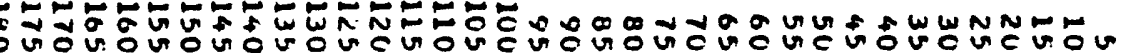
:

至

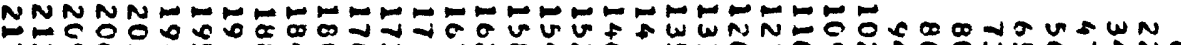

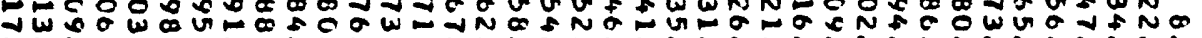

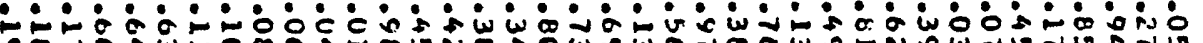

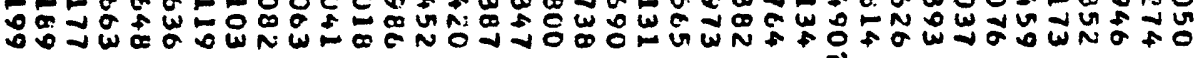

00000000000000000000000000000000000000

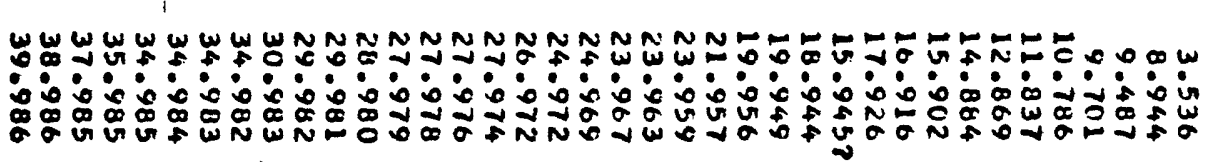

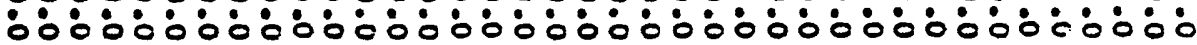




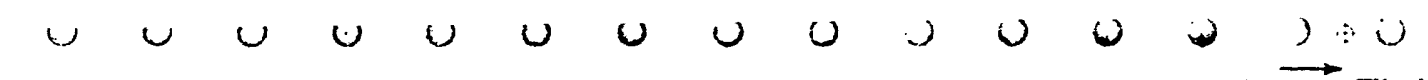
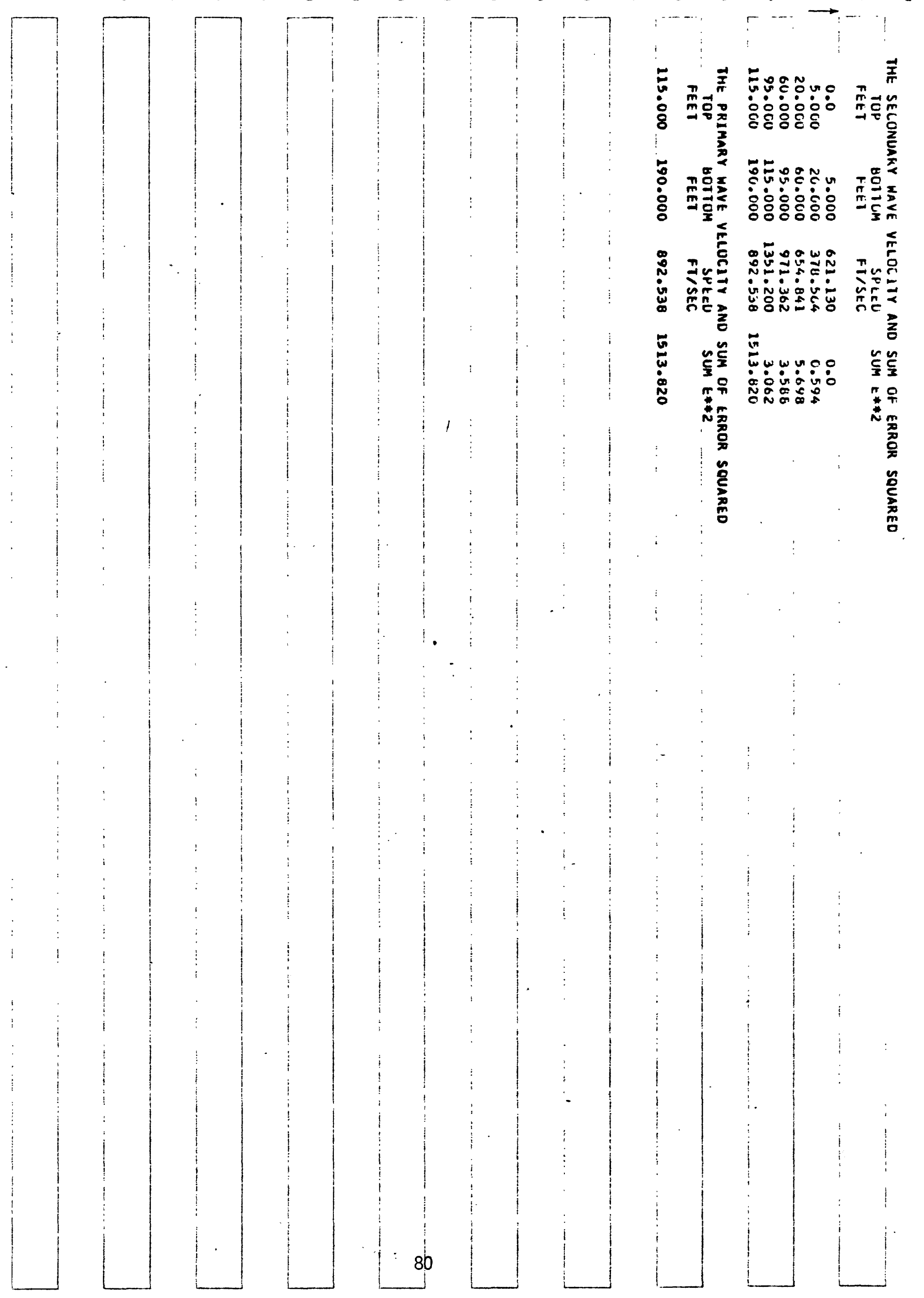
ASPHALT - COARSE INTERPRETATION MODEL II

FIGURES 15 AND 16 


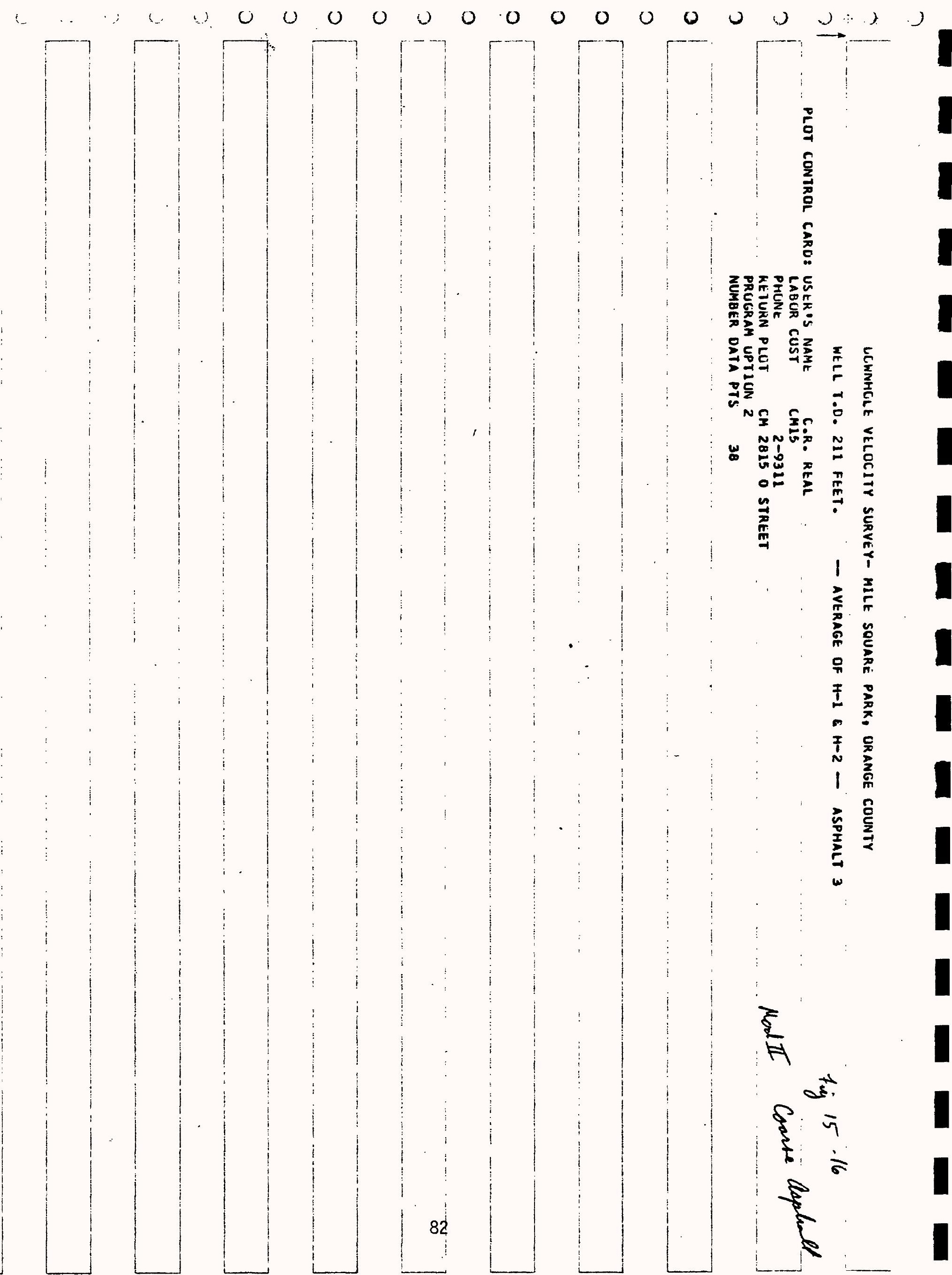




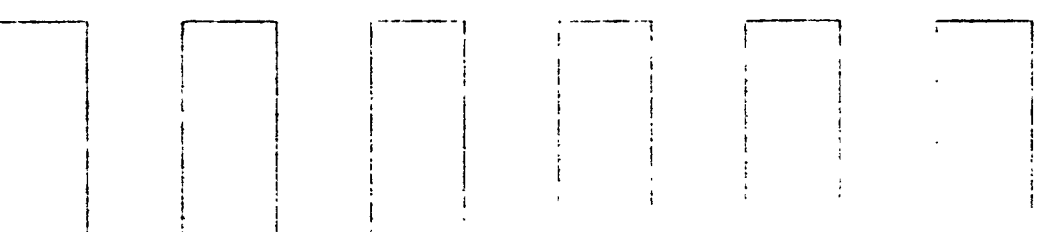

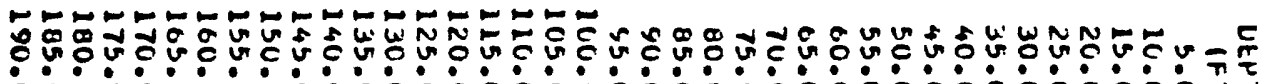

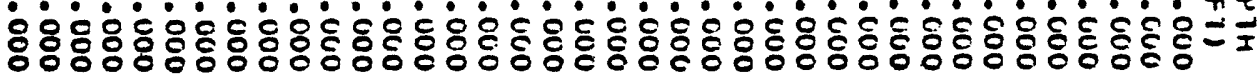

号王

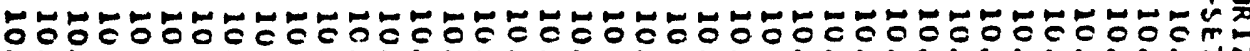

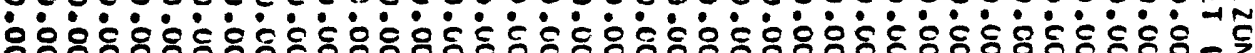

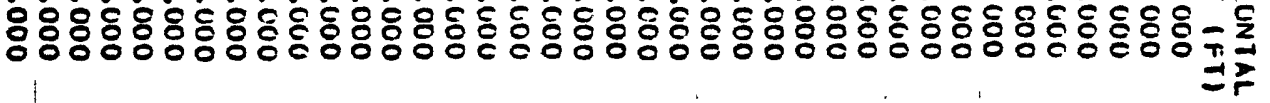

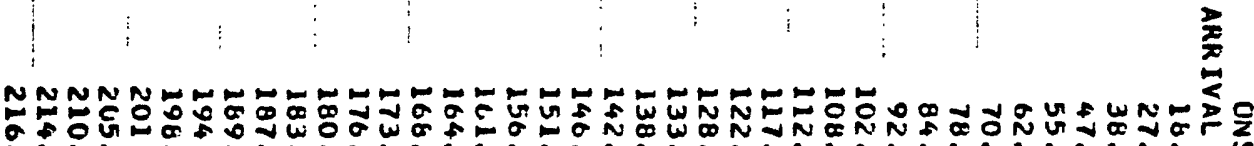

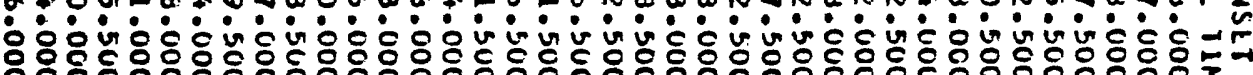

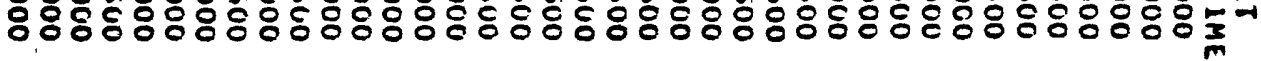

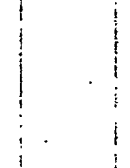

$00000000000000000000000000000000000000 \$ \$$ $\dot{0} \dot{0} \dot{0} \dot{0} \dot{0} \dot{0} \dot{0} \dot{0} \dot{0} \dot{0} \dot{0} \dot{0} \dot{0} \dot{0} \dot{0} \dot{0} \dot{0} \dot{0} \dot{0} \dot{0} \dot{0} \dot{0} \dot{0} \dot{0} \dot{0} \dot{0}=\frac{\mathrm{F}}{\mathrm{T}}$ $00000000000000000000000000000000000000^{\frac{1}{3}}$ $\bar{z}$

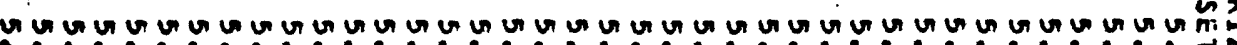

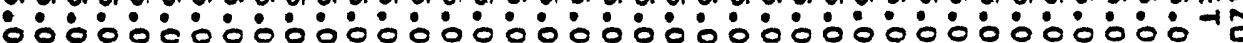

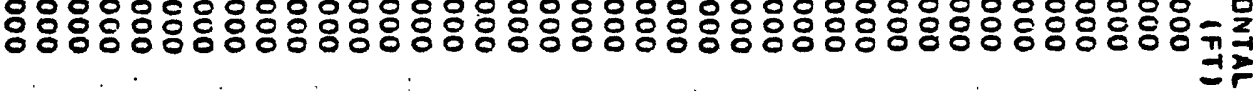

○

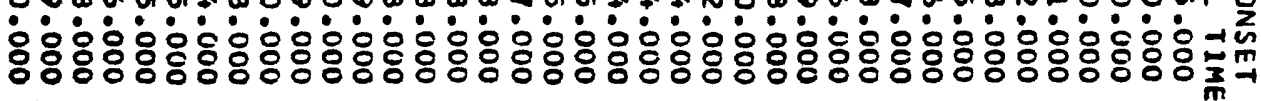


$\begin{array}{lllllllllllllll}0 & 0 & 0 & 0 & 0 & 0 & 0 & 0 & 0 & 0 & 0 & 0 & 0 & 0 & \ddots\end{array}$
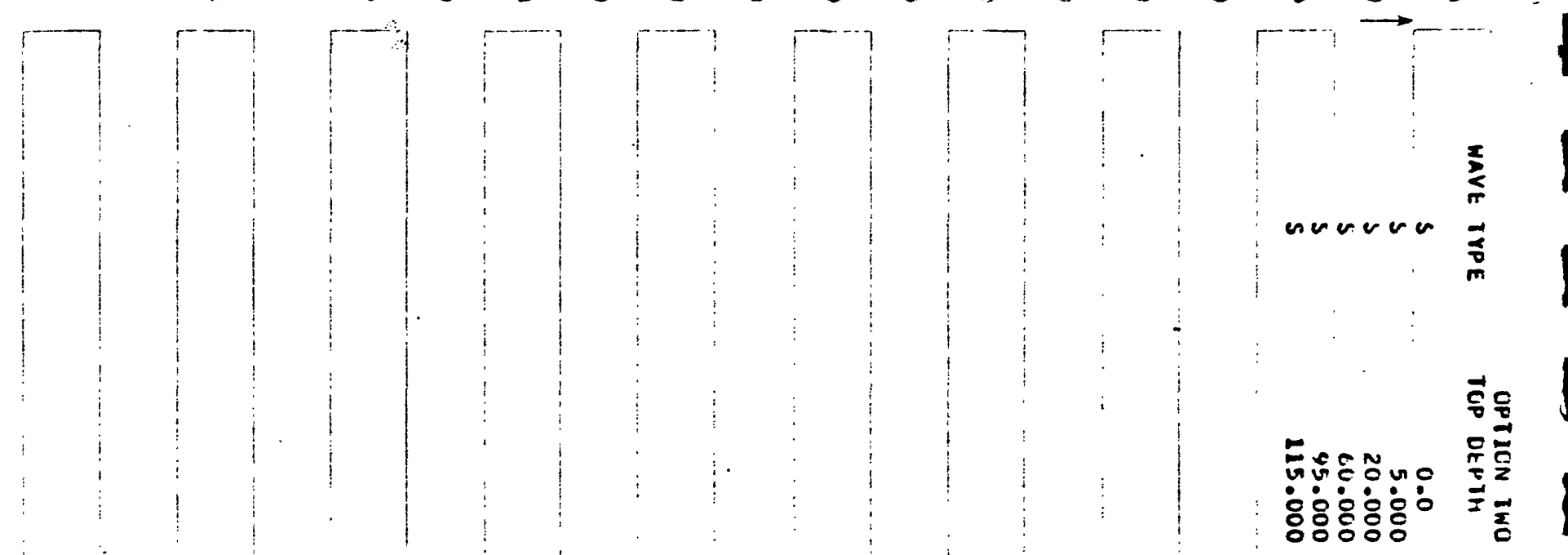

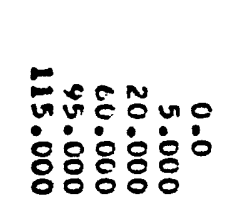

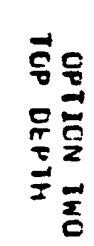

ธำํํำ

ถั่ํํำ

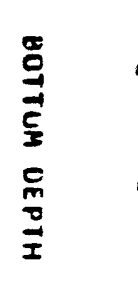


$\begin{array}{lllllllllllll}0 & 0 & 0 & 0 & 0 & 0 & 0 & 0 & 0 & 0 & , & ,\end{array}$

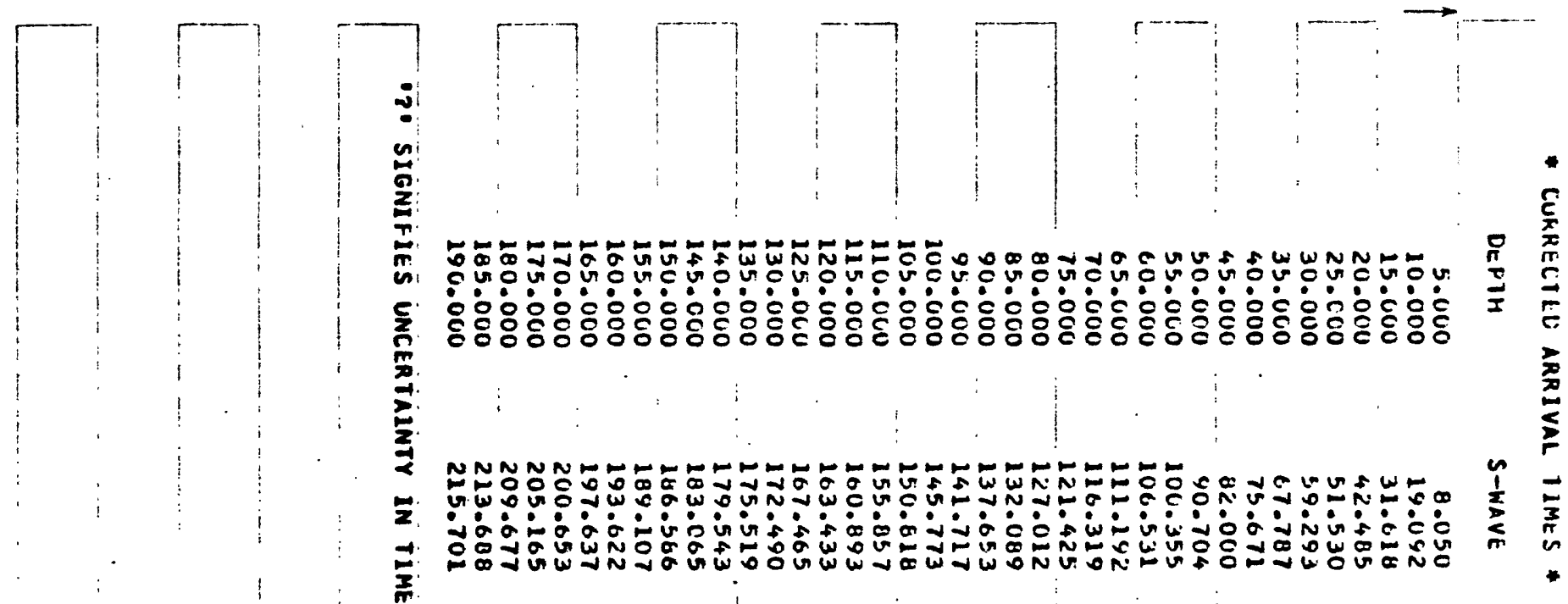

WWWWWWWW W

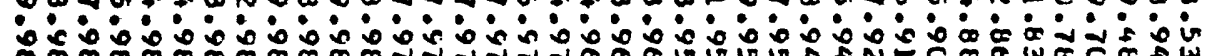

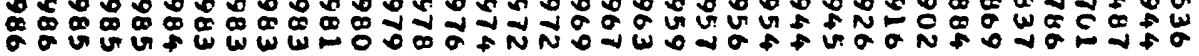

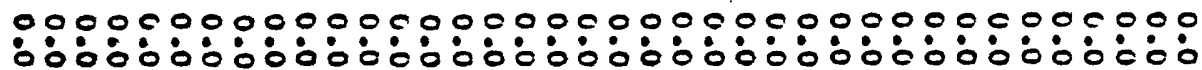




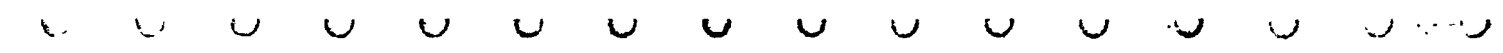

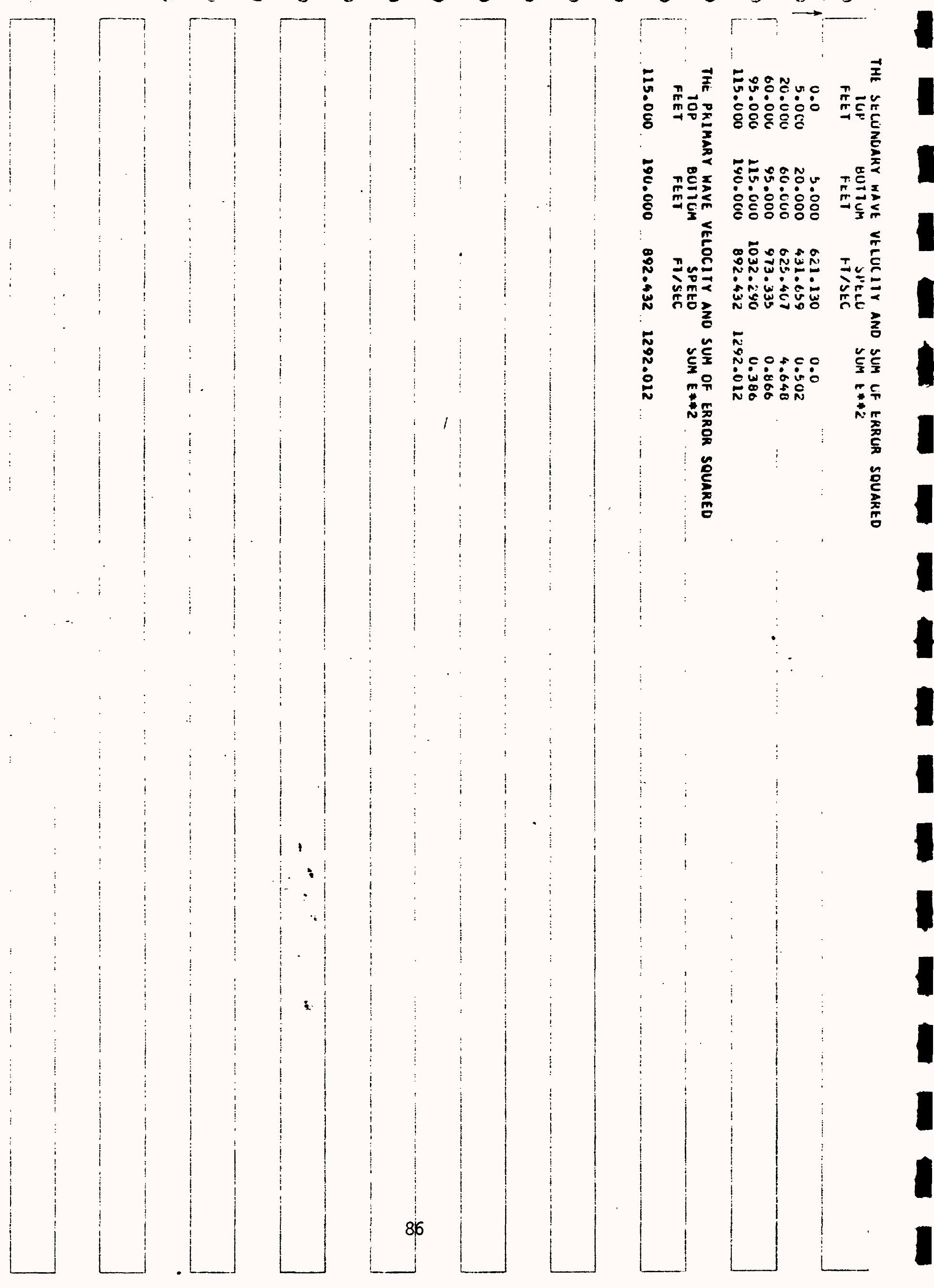


S U P P L EMENT TO THE

A N N U A TECHNICAL REPORT

F O R

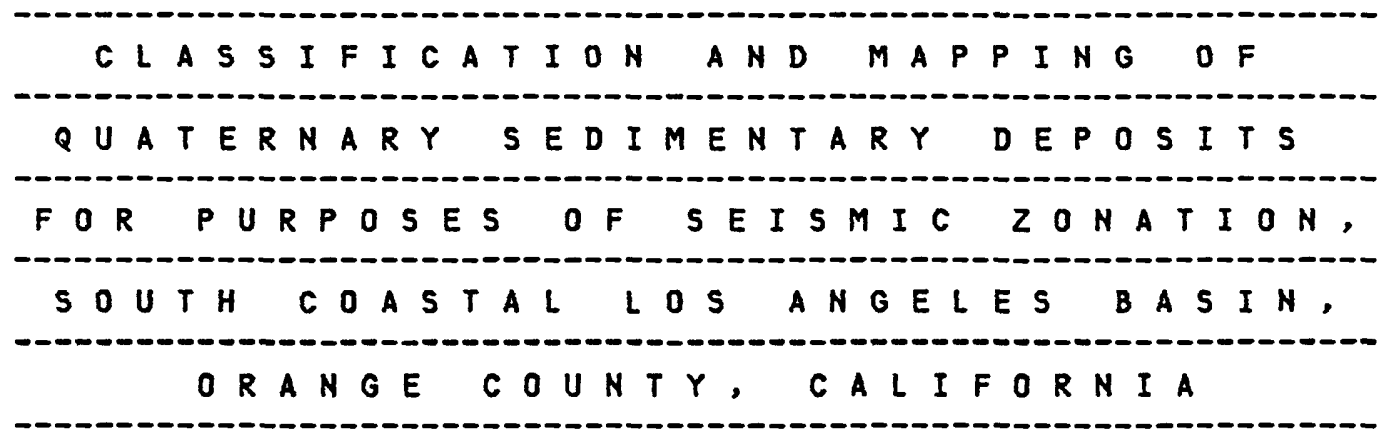

\author{
DESCRIPTION OF THE \\ ELECTRONIC DATA PROCESSING \\ CAPABILITY DEYELOPED TO MANAGE \\ STRATIGRAPHIC SAMPLE DATA \\ AND GEOTECHNICAL MEASUREMENTS
}

\begin{abstract}
AUTHOR ....... HENRY A. MUMM, PROGRAMMER/ANALYST
CALIFORNIA DEPARTMENT OF CONSERVATION

DIVISION OF MINES AND GEOLOGY

1416 9TH STREET, ROOM 1326-2

SACRAMENTO, CALIFORNIA 95814

(916) 323-1360
\end{abstract}

DATE ........ SEPTEMBER, 1980

SPONSORED BY .. U. S. GEOLOGICAL SURVEY

(CONTRACT NO. 14-08-0001-18241)

COUNTY OF ORANGE, CALIFORNIA,

ENVIRONMENTAL MANAGEMENT AGENCY

(COOPERATIVE AGREEMENT NO. 5-0052)

87 (p. 89 follows) 
1. GENERAL SYSTEM DESCRIPTION $\ldots \ldots \ldots \ldots \ldots \ldots \ldots \ldots \ldots$. 1

2. EDIT/AUDIT/CHANGE FUNCTIONAL DESCRIPTION ...(INPUT)... 2

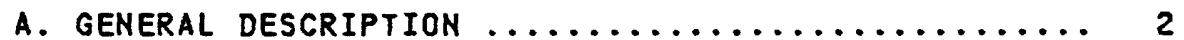

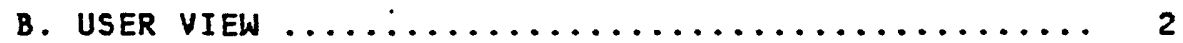

C. INTERNAL/TECHNICAL VIEW $\ldots \ldots \ldots \ldots \ldots \ldots \ldots \ldots \ldots \ldots \ldots \ldots \ldots \ldots$

3. DATA RETRIEVAL FUNCTIONAL DESCRIPTION ... (OUTPUT).... 9

A. GENERAL DESCRIPTION ....................

B. USER VIEW ..........................

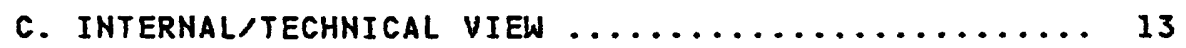

4. DATA SECURITY GONSIDERATIONS $\ldots \ldots \ldots \ldots \ldots \ldots \ldots \ldots \ldots$

ATTACHMENTS

A. SAMPLE OF THE INPUT FORM FOR GEOTECHNICAL DATA ..... Al Al $\rightarrow$

B. SAMPLE STRATIGRAPHIC EDIT/AUDIT AND CHANGE REPORTS . B B1-11 -->

C. DATA EDIT AUdit DEFInITION $: \ldots \ldots \ldots \ldots \ldots \ldots \ldots \ldots \ldots$. $18 \ldots \ldots$

D. QUERY CONTROL SERUICES MENU $\ldots \ldots \ldots \ldots \ldots \ldots \ldots \ldots$ DI $\quad \rightarrow$

E. STRATIGRAPHIC dATA NAME MENU $\ldots \ldots \ldots \ldots \ldots \ldots \ldots \ldots$ El-49 $\rightarrow$.

F. STRATIGRAPHIC OUTPUT SERVICE MENU $\ldots \ldots \ldots \ldots \ldots \ldots \ldots$ FI-9 - .

G. SAMPLE QUERIES AND THE RESULTING STRATIGRAPHIC PROPERTIES AND DATA REPORTS ........GI-22 -->

H. ADDITIONAL QUERY EXAMPLES $\ldots \ldots \ldots \ldots \ldots \ldots \ldots \ldots \ldots$ HI-9 $\rightarrow \ldots$ 
THE DATA PROCESSING SYSTEM DESCRIBED IN THE FOLLOWING PAGES WAS DESIGNED TO ENABLE THE STORAGE AND SELECTIVE RETRIEVAL OF DATA ABOUT GEOLOGIC SAMPLES AND MEASUREMENTS TAKEN FROM HOLES IN THE GROUND. THE DATA INCLUDE SUCH INFORMATION AS THE LOCATION OF THE SAMPLE HOLE, CHARACTERISTICS OF THE LITHOLOGIC LAYER FROM WHICH THE SAMPLE WAS TAKEN, AND THE SPECIFIC PROPERTIES OF THE SAMPLE.

THE CURRENT SYSTEM CAN ONLY STORE POINT DATA--THAT IS, DATA REPRESENTING A MEASURED VALUE AT SOME POINT IN SPACE AND TIME AS OPPOSED TO DATA REPRESENTING A MEASUREMENT BETWEEN TWO POINTS SUCH AS THE TOP AND BOTTOM OF A LAYER. THE SYSTEM AS DESIGNED DOES NOT, HOWEVER, PRECLUDE ADDING RANGE DATA AT SOME FUTURE TIME.

THE SYSTEM CONSISTS OF TWO MAJOR FUNCTIONS, AN EDIT/AUDIT/CHANGE FUNCTION (INPUT) AND DATA RETRIEVAL FUNCTION (OUTPUT).

THE EDIT/AUDIT/CHANGE FUNCTION ASSURES THAT THE ONLY DATA THAT IS USABLE AND CONSISTENT WITH USER SPECIFIED REQUIREMENTS REACHES THE STORAGE FILE. THE DATA RETRIEVAL FUNCTION PROVIDES A VARIETY OF SERVICES RELATED TO OBTAINING AND DISPLAYING INFORMATION FROM THE STORAGE FILE. THE EDIT/AUDIT/CHANGE FUNCTION IS DRIVEN BY A USERCODED INPUT FORM, WHILE THE DATA RETRIEVAL FUNCTION IS DRIVEN BY A USER-CODED QUERY.

THE DATA ABOUT THE GEOLOGIC SAMPLES IS ORGANIZED INTO THREE GROUPS

1) DATA ABOUT THE SURFACE LOCATION OF THE HOLE.

2) DATA. ABOUT THE LITHOLOGIC LAYER FROM WHICH THE SAMPLE WAS TAKEN.

3) DATA ABOUT THE SPECIFIC PROPERTIES OF THE SAMPLE TAKEN.

INDIVIDUAL PIECES OF DATA ARE REFERRED TO AS DATA ITEMS. A DATA ITEM IS THE SMALLEST UNIT OF DATA TO WHICH A NAME IS GIVEN AND TO WHICH MEANING IS ASCRIBED. DATA ITEMS ARE SUCH THINGS AS DAY OF MONTH, YEAR, STATE, DEPTH, METHOD, GENERAL LITHOLOGY, ETC.

DATA ITEMS ARE STORED BASED ON A FIVE DIMENSIONAL MODEL. THE FIVE DIMENSIONS USED ARE

1) THE ZONE AND EAST VALUE OF THE CALIFORNIA COORDINATE SYSTEM.

2) THE ZONE AND NORTH VALUE OF THE CALIFORNIA COORDINATE SYSTEM.

3) THE DEPTH BELOW THE SURFACE. (ZERO IS A VALID VALUE.)

4) THE TIME IN THE FORM OF A DATE APPROPRIATE TO THE GROUP TO WHICH A DATA ITEM IS ASSIGNED.

5) THE GROUP TO WHICH THE DATA ITEM BELONGS.

TAKE FOR EXAMPLE THE STORING OF THE MEASURED DEPTH OF THE WATER TABLE. SPACIALLY THE LOCATION $(X, Y, Z)$ IS THE FIRST THREE DIMENSIONS. THE SAMPLE TO DETERMINE THE DEPTH OF THE WATER TABLE IN SOME HOLE WAS TAKEN ON SOME DATE, THE FOURTH DIMENSION. THE FIFTH DIMENSION IS THAT THIS MEASURMENT IS THE WATER TABLE. 


\section{GENERAL DESCRIPIION}

THE EDIT/AUDIT/CHANGE FUNCTION IS DESIGNED TO PROVIDE THE USER WITH MESSAGES ABOUT SPECIFIC CONDITIONS DETECTED WHEN A USER-CODED INPUT FORM IS PROCESSED. A SAMPLE OF THE INPUT FORM IS SHOWN AS ATTACHMEMT A. SAMPLES OF THE EDIT/AUDIT/CHANGE REPORT ARE SHOWN AS ATTACHMENT B. THE MAJORITY OF THE VALUES USED FOR PURPOSES OF EDITING AND AUDITING ARE SHOWN AS ATTACHMENT $C$.

A DISTINCTION IS MADE BETWEEN TWO GENERIC TYPES OF DATA ITEMS. KEY DATA ITEMS ARE THOSE NECESSARY TO UNIQUELY IDENTIFY THE DATA TO BE OPERATED ON. DETAIL DATA ITEMS ARE THOSE WHICH PROVIDE INFORMATION ABOUT THE SPECIFIC KEY WITH WHICH THESE DATA ITEMS ARE ASSOCIATED. THE KEY GIVES SPECIFIC IDENTITY AND MEANING TO THE DETAIL DATA ITEMS. THE KEY DATA ITEMS ARE HIGH-LIGHTED ON THE INPUT FORM.

WITH THE EXCEPTION OF KEY DATA ITEMS AND THE DATA SOURCE INFORMATION, THE ENTRY OF A VALUE FOR ANY DATA ITEM IS OPTIONAL. THE DESIGN PERMITS NEW DATA ITEMS OR VALUES FOR EXISTING DATA ITEMS TO BE ADDED TO THE STRATIGRAPHIC STORAGE FILE AS THEY ARE IDENTIFIED.

\section{USER VIEW}

WHEN PREPARING AN INPUT FORM, THE USER MUST SPECIFY ONE OF FOUR ACTIONS FOR ALL DETAIL DATA ITEM VALUES. THE FOUR ACTIONS ARE ADD, DELETE, UPDATE AND REPLACE. THE DESIRED ACTION MAY BE ENTERED IN TWO WAYS. FIRST AS A GENERAL ACTION. THE GENERAL ACTION IS REQUIRED AND APPLIES TO ALL DATA ITEMS ON AN INPUT FORM FOR WHICH A VALUE IS ENTERED. THE GENERAL ACTION FOR ANY SPECIFIC DATA ITEM MAY BE OVERRIDDEN BY ENTERING A SPECIFIC ACTION FOR THAT DATA ITEM. THE SPECIFIC ACTION FIELD APPEARS FOLLOWING EACH DATA ITEM AND IS IDENTIFIED BY ENCLOSING IT IN PARENTHESIS, I.E "(_)". ALL DETAIL DATA ITEMS ARE OPTIONAL AFTER THE INITIAL ENTRY OF THE PRIMARY DATA SOURCE. ALL FOUR ACTIONS MAY BE ENTERED ON ANY INPUT FORM. KEY DATA ITEMS

- FOR SURFACE DATA ARE:

+ CALIFORNIA COORDINATES

+ DATE OF SEGMENT (DEFAULT IS THE DATE OF THE RUN)

- FOR LITHOLOGIC LAYER DATA ARE:

+ CALIFORNIA COORDINATES

+ DEPTH TO TOP

+ DATE OF SEGMENT (DEFAULT IS THE DATE OF THE RUN)

- FOR LITHOLOGIC SAMPLE DATA ARE:

+ CALIFORNIA COORDINATES

+ DEPTH TO SAMPLE (ZERO IS A VALID VALUE)

+ DATE OF SAMPLE

+ PARAMETER CODE 
THE STRATIGRAPHIC DATA EDIT/AUDIT AND CHANGE REPORT REPRODUCES THE USER-CODED INPUT FORM ON THE LEFT-HAND SIDE OF THE PAGE AND LISTS ANY MESSAGES ON THE RIGHT. ASSOCIATED WITH EACH MESSAGE IS A REFERENCE CODE. THIS REFERENCE CODE IDENTIFIES THE DATA ITEM VALUE TO WHICH THE MESSAGE APPLIES BY APPEARING UNDER THE DATA ITEM VALUE. AN ARROW IS PLACED TO THE IMMEDIATE LEFT OF THE COLUMN FOR THE REFERENCE CODES TO INDICATE WHICH LINES WITHIN THE REPORT CONTAIN THE REFERENCE CODES.

EACH MESSAGE SPECIFIES THE CONDITION DETECTED. CONDITIONS WHICH GENERATE MESSAGES INCLUDE A NONNUMERIC, INVALID OR OUT-OFRANGE VALUE, A VALUE THAT WAS COMPUTER GENERATED, TWO OR MORE VALUES ARE INCONSISTENT, AND A VALUE WAS FOUND ON FILE DURING AN ADD OPERATION. FOR THOSE CONDITIONS WHICH PREVENT THE SPECIFIED ACTION FROM BEING COMPLETED, A MESSAGE IS PRINTED GIVING THE DISPOSITION OF THE DATA ITEM VALUE INVOLVED. 


\section{INTERNAL/TECHNICAL VIEW}

PROGRAM DESIGN CONCEPT:

THE EDIT/AUDIT/CHANGE FUNCTION IS DESIGNED TO COLLECT ALL THE SEGMENTS FOR AN "INPUT FORM", PASS EACH SEGMENT THROUGH APPROPRIATE EDITS AND AUDITS, CHANGE THE STORAGE FILE AS INDICTED BY THE ACTION CODES IF NO MAJOR ERRORS WERE DETECTED, AND WRITE THE EDIT/AUDIT/CHANGE REPORT.

ANY OF THREE DIFFERENT SEGMENT TYPES--SURFACE, LAYER OR PARAMETER-MAY BE GENERATED BY AN INPUT FORM. AN INPUT FORM MAY GENERATE ONE SURFACE, ONE LAYER OR SEVERAL PARAMETER SEGMENTS. EACH PARTICULAR SEGMENT TYPE, HOWEVER, IS OPTIONAL.

AN "INPUT FORM" IS "ALL THE PARAMETER SEGMENTS FOR A DEPTH TO SAMPLE". AN "INPUT FORM" MAY ALSO CONTAIN NO MORE THAN ONE CALIFORNIA COORDINATE VALUE, GENERAL ACTION VALUE, SURFACE OR LAYER SEGMENT. THE DEPTH TO TOP OF A LAYER SEGMENT MAY BE LESS THAN, EQUAL TO OR GREATER THAN THE DEPTH OF SAMPLE ON THE SAME INPUT FORM. ALL SEGMENTS FOR AN INPUT FORM MUST HAVE THE SAME CALIFORNIA COORDINATES AND GENERAL ACTION CODE. IF A DUPLICATE SEGMENT IS FOUND, BOTH ARE REPORTED AND DISCARDED.

FIRST, FOR EACH SEGMENT TYPE THE FOLLOWING PROCESSING STEPS ARE DONE:

1) INITIALIZE ANY CONTROL INDICATORS OR OTHER DATA ITEMS NEEDED FOR PROCESSING THIS SEGMENT TYPE.

2) TAKE THE STATISTICAL COUNTS FOR THIS SEGMENT TYPE.

3) PREPARE THE DATA ITEMS UNIQUE TO THIS SEGMENT TYPE FOR EDITING AND AUDITING. THIS INVOLVES TRANSFERING EACH DATA ITEM'S VALUE FROM THE PHYSICAL INPUT DEFINITION TO THE INTERNAL EDIT DEFINITION. LEADING ZEROES MAY BE ADDED TO A RECEIVED VALUE TO PREPARE IT FOR EDITING.

4) EDIT THE SEGMENT DATA ITEM VALUES RECEIVED. AN "EDIT" IS DEFINED AS AN INTRAFIELD CHECK.

5) AUDIT THE SEGMENT DATA ITEM VALUES WHICH PASSED THE EDITS. AN "AUDIT" IS DEFINED AS AN INTERFIELD CHECX.

6) STORE THE IMAGE OF THE PHYSICAL SEGMENT TO BE USED AS THE SOURCE FOR THE DATA VALUES TO BE SHOWN ON THE EDIT/ AUDIT/CHANGE REPORT.

7) IF NO MAJOR ERRORS WERE DETECTED TRANSFER ALL VALID DATA ITEM YALUES FROM THE EDIT DEFINITION TO THE LOGICAL DEFINITION FOR LATER PROCESSING AGAINST THE STORAGE FILE. 


\begin{abstract}
SECOND,
FOR EACH SEGMENT TYPE TO COMPLETE THE CHANGES SPECIFIED TO THE

STORAGE FILE THE FOLLOWING PROCESSING STEPS ARE DONE:

1) IF THE INPUT FORM HAS A SURFACE SEGMENT AND THE SURFACE DATA CAN BE CHANGED, MAKE THE CHANGES SPECIFIED.

2) IF THE INPUT FORM HAS A LAYER SEGMENT AND THE LAYER DATA CAN BE CHANGED, MAKE THE CHANGES SPECIFIED.

3) IF THE INPUT FORM HAS A PARAMETER SEGMENT AND THE PARAMETER DATA CAN BE CHANGED, MAKE THE CHANGES SPECIFIED.
\end{abstract}

FOR EACH SEGMENT, REGARDLESS OF THE TYPE, THAT SPECIFIES CHANGES TO THE STORAGE FILE THE FOLLOWING PROCESSING STEPS ARE DONE:

1) OBTAIN THE DESIRED SEGMENT FROM THE STORAGE FILE BY POSITIONING THE FILE AT THE KEY OF THE SEGMENT TO BE CHANGED. IF NO KEY IS FOUND A NEW SEGMENT IS CREATED.

2) THE ACTIONS ARE APPLIED TO THE SEGMENT OBTAINED ABOVE IN THE ORDER OF DELETIONS, ADDITIONS, REPLACEMENTS AND UPDATES.

FOLLOWING THIS THE EDIT/AUDIT/CHANGE REPORT IS WRITTEN. THE FORMAT FOR THE REPORT IS DIVIDED INTO "REPORT BLOCKS" WHICH ARE PERFORMED ON AN AS NEEDED BASIS. THESE REPORT BLOCKS ARE ALSO USED TO FORMAT SUCH CONDITIONS AS DUPLICATE SEGMENTS AND PAGE OVERFLOW FOR AN INPUT FORM. THE MAIN REPORT BLOCKS LOGICALLY ASSOCIATE WITH THE SEGMENT TYPES. OTHER REPORT BLOCKS BUILD AND WRITE AREAS LIKE THE REPORT TITLES.

PROGRAM DESIGN METHODOLOGY:

STRUCTURED PROGRAM DESIGN AS OFFERED BY KEN ORR AND ASSOCIATES, INC. OF TOPEKA, KANSAS WAS THE METHODOLOGY USED FOR THE DESIGN OF THE EDIT/AUDIT/CHANGE PROGRAM.

PROGRAMMING LANGUAGE:

THE EDIT/AUDIT/CHANGE PROGRAM IS WRITTEN USING IBM COBOL. KNOWN NON-STANDARD COBOL IS USED TO OBTAIN THE COMPILE DATE AND TIME FROM THE "WHEN-COMPILED" DATA NAME. THE DATE AND TIME OF THE START AND END OF THE RUN IS OBTAINED USING THE "DATE" AND "TIME-OF-DAY" DATA NAMES. ALSO USED IS A SUBROUTINE CALLED "DHSORT" WHICH IS AN INTERNAL TABLE SORT. FOR ANY ONE WHO WISHES TO INSTALL AND USE THIS PROGRAM IT IS SUGGESTED THAT AN ATTEMPT BE MADE TO COMPILE THE PROGRAM AND SEE WHAT ERRORS RESULT. 


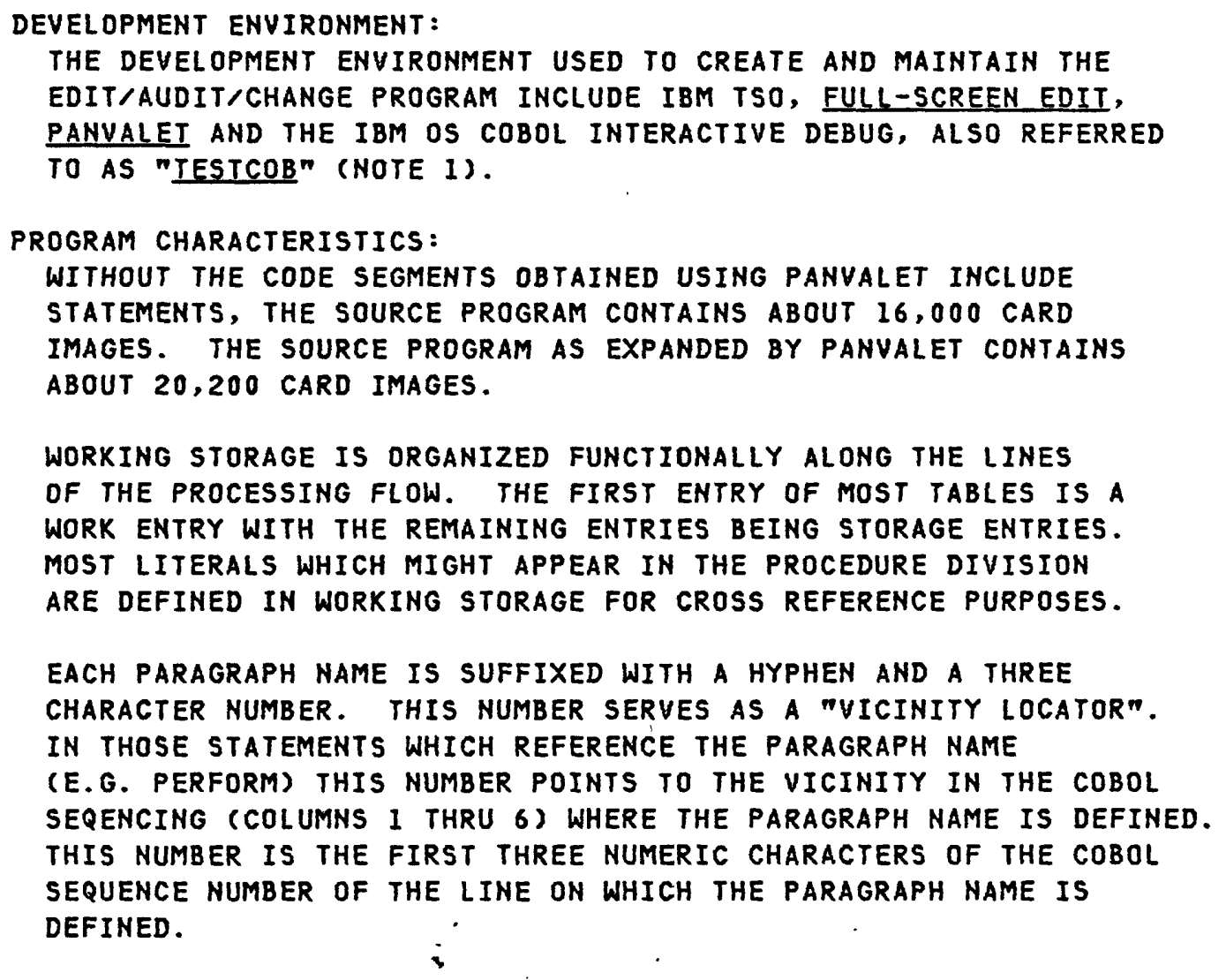

NOTE 1: FULL-SCREEN EDIT IS A PROPRIETARY SOFTWARE PRODUCT FROM APPLIED SOFTWARE, INC.

P. O. BOX 342176

CORAL GABLES, FLORIDA 33134

COPYRIGHT - APPLIED SOFTWARE, INC. 1977,1979

PANVALET ...... IS A PROPRIETARY SOFTWARE PRODUCT FROM
PANSOPHIC SYSTEMS, INCORPORATED
1301 WEST 22ND STREET
OAK BROOK, ILLINOIS 60521
COPYRIGHT - PANSOPHICS SYSTEMS, INC.
1975




\author{
DATA FILE CHARACTERISTICS: \\ CHANGE .... THE INPUT CHANGE FILE CONTAINS DATA SEGMENTS TO BE \\ PROCESSED AGAINST THE STORAGE FILE. THE CHANGE \\ SEGMENTS ARE 84 CHARACTERS IN LENGTH AND ARE CREATED \\ FROM THE INPUT FORM BY KEY ENTRY. ANY OF THREE \\ DIFFERENT SEGMENT TYPES--SURFACE, LAYER OR PARAMETER-- \\ MAY BE GENERATED BY AN INPUT FORM. AND INPUT FORM \\ MAY GENERATE ONE SURFACE, ONE LAYER OR SEVERAL \\ PARAMETER SEGMENTS. EACH PARTICULAR SEGMENT TYPE, \\ HOWEVER, IS OPTIONAL. THE CURRENT PROGRAM LIMIT FOR \\ PARAMETER SEGMENTS IS 44 PER "INPUT FORM" REPORTED \\ ON THE EDIT/AUDIT/CHANGE REPORT. \\ FOR THE PURPOSES OF THE PROGRAM, A SAMPLE IS ALL THE \\ PARAMETER SEGMENTS FOR A DEPTH TO SAMPLE. THESE \\ PARAMETER SEGMENTS MAY ORIGINATE FROM DIFFERENT USER- \\ CODED INPUT FORMS. \\ STORAGE ... THE STORAGE FILE IS A SEQUENTIAL FILE AND CONTAINS \\ DATA SEGMENTS WHICH MIRROR THOSE OF THE INPUT CHANGE \\ FILE STRUCTURE DESCRIBED ABOVE. THE STORAGE SEGMENTS \\ ARE 96 CHARACTERS IN LENGTH AND ARE CREATED OR CHANGED \\ UPON THE VERIFICATION OF AN CHANGE SEGMENT. ANY \\ SEGMENT WHICH CONTAINS ONLY KEY DATA ITEMS IS \\ AUTOMATICALLY DELETED. THUS, THE STORAGE FILE IS \\ "SELF. COMPRESSING". \\ REPORT ... THERE ARE THREE REPORT FILES GENERATED. \\ 1) THE STRATIGRAHIC EDIT/AUDIT AND CHANGE REPORT. \\ 2) THE USER RUN PROFILE WHICH PROVIDES STATISTICAL \\ INFORMATION TO THE USER. \\ 3) THE RUN CONTROLS REPORT WHICH PROVIDES STATISTICAL \\ INFORMATION TO THE SYSTEM PERSONNEL WHICH MAKE \\ THE PROCESSING RUNS. \\ ALL REPORT FILE PRINT LINES ARE 133 CHARACTERS IN \\ LENGTH WITH THE FIRST CHARACTER BEING A CARRIAGE \\ CONTROL CHARACTER.
}


SUPPORT ... THE PROGRAM REQUIRES THREE SUPPORT FILES.

1) THE APPLICATION SUPPORT INDEX.

THIS FILE SERVES AS AN INDEX FOR SUPPORT FILES TWO AND THREE.

2) THE APPLICATION MESSAGE FILE. THIS FILE SERVES AS THE SOURCE FOR MESSAGE TEXT FOR DEPARTMENT APPLICATIONS. TO OBTAIN THE CORRECT MESSAGE NUMBERS FOR THIS APPLICATION THE APPLICATION SUPPORT INDEX MUST BE USED.

3) THE DATA SOURCE SUPPORT FILE. THIS FILE SERVES AS THE SOURCE FOR VERIFING THE PRIMARY AND SECONDARY DATA SOURCES ENTERED ON THE INPUT FORM. THIS FILE ALSO PROVIDES THE MINIMUM RELEASE AUTHORITY FOR EACH DATA SOURCE AND ACTIVITY. TO OBTAIN THE CORRECT DATA SOURCE CODES FOR THIS APPLICATION THE APPLICATION SUPPORT INDEX MUST BE USED. THIS FILE IS UNIQUE TO EACH INSTALLATION. IT IS REQUIRED TO PROCESS ANY CHANGE SEGMENTS CORRECTLY AND MUST BE CREATED PRIOR TO ANY CHANGE RUNS.

A METHOD TO CREATE THE APPLICATION SUPPORT INDEX AND DATA SOURCE SUPPORT FILES IS PROVIDED WITH A -REQUEST FOR THE PROGRAM SOURCE CODE. THE APPLICATION MESSAGE FILE FOR THIS PROGRAM IS ALSO PROVIDED.

TESTING:

THE TEST SYSTEM DEVELOPED FOR THE EDIT/AUDIT/CHANGE FUNCTION FULFILLS FOUR DESIRABLE CHARACTERISTICS:

1) THE TEST CASE MODEL REFLECTS THE CONTENT AND STRUCTURE OF AN INPUT FORM.

2) THE TEST CASE MODEL IS EASILY USED BY HUMAN BEING. TWO KINDS OF TEST CASE MODELS WERE DEVELOPED. FIRST, A MODEL FOR USE WITH A CRT TYPE FULL-SCREEN TEXT PROCESSING CAPABILITY. AND SECOND, A MODEL FOR USE WITH A LINE-AT-ATIME TEXT PROCESSING CAPABILITY.

3) THE COMPLETED TEST CASE IS MACHINE PROCESSABLE IN THAT IT CAN BE CONVERTED DIRECTLY TO THE PHYSICAL CHANGE FILE.

4) EACH TEST CASE CAN BE PRINTED ON STANDARD $81 / 2 \times 11 \mathrm{INCH}$ PAPER FOR DOCUMENTATION.

TEST CASES MAY BE GROUPED INTO SETS SO THAT DIFFERENT KINDS OF TESTS MAY BE RUN. ANY TEST CASE MAY BE INCLUDED AS PART OF ONE OR MORE SETS OF TEST CASES WITHOUT THE NECESSITY OF DUPLICATING THE TEST CASE. ALSO ANY SET OF TEST CASES MAY BE INCLUDED AS PART OF ONE OR MORE SETS OF TEST CASES. PANVALET IS USED TO ACHIEVE THIS FLEXIBILITY. A SOME WHAT LESS FLEXIBLE ORGANIZING SYSTEM FOR TEST CASES CAN BE ACHIEVED USING THE COBOL COPY FACILITY. THIS TEST SYSTEM CAPABILITY WILL BE PROVIDED AS PART OF THE SYSTEM IF REQUESTED. 


\section{GENERAL DESCRIPTION}

THE DATA RETRIEVAL FUNCTION IS DESIGNED TO PROVIDE LIMITED AD HOC QUERY PROCESSING CAPABILITY USING MENUS AS THE BASE FOR CODING THE QUERY. THREE MENUS ARE PROVIDED:

1) QUERY CONTROL SERVICES MENU. (ATTACHMENT D)

2) STRATIGRAPHIC DATA NAME MENU. (ATTACHMENT E)

3) STRATIGRAPHIC OUTPUT SERVICES MENU. (ATTACHMENT F)

THE CODING OF A QUERY IS DONE BY FIRST SPECIFYING WHICH QUERY CONTROL SERVICES ARE DESIRED FOR PROCESSING THIS QUERY. THE "IF" OR CONDITION PORTION OF THE QUERY IS THEN CODED UTILIZING DATA NAMES SELECTED FROM THE STRATIGRAPHIC DATA NAME MENU. (THE CONDITION PORTION OF THE QUERY IS OPTIONAL.) FOLLOWING THE CONDITION PORTION OF THE QUERY, THE OUTPUT SERVICES DESIRED ARE CODED. AS OF THIS WRITING OUTPUT IS LIMITED TO THE "PRINT" SERVICES. THE "PLOT" SERVICES ARE EXPECTED TO BE IMPLEMENTED SHORTLY. ONLY ONE QUERY CAN BE PROCESSED DURING ANY RUN OF THE RETRIEVAL PROCESS. ONE RUN MUST BE DONE FOR EACH QUERY DESIRED.

SAMPLE QUERIES AND THE RESULTING STRATIGRAPHIC PROPERTIES AND DATA REPORTS ARE PROVIDED AS ATTACHMENT G. ADDITIONAL QUERY EXAMPLES ARE PROVIDED AS ATTACHMENT H.

AS IMPLEMENTED AT THE CALIFORNIA DEPARTMENT OF CONSERVATION, A VALUE IS REQUIRED FOR THE "RELEASE-AUTHORITY-AUTHORIZED" OPTION OF THE CONTROL SERVICES MENU FOR ALL QUERIES PROCESSED. THE CONTROL WAS IMPLEMENTED AS PART OF THE DATA SECURITY. PLEASE SEE THE SECTION ON DATA SECURITY CONSIDERATIONS FOR A FURTHER DISCUSSION.

\section{USER VIEW}

THE USER'S VIEW OF THE DATA RETRIEVAL CAPABILITY CONSISTS ENTIRELY OF THE CODED QUERY AND THE DATA RETRIEVAL PROCESS'S RESPONSE TO THE CODED QUERY. DESCRIPTION OF THE CODING OF A QUERY IS BEST DONE BY EXAMPLE.

IN ONE OF ITS SIMPLEST FORMS, THE CONDITION PORTION OF A CODED QUERY WOULD CONSIST OF ONE OR MORE DATA NAMES SELECTED FROM THE DATA NAME MENU, THE COMPARISON OF THEM WITH SOME VALUE AND LOGICALLY ASSOCIATING THEM TOGETHER WITH AN "AND". 


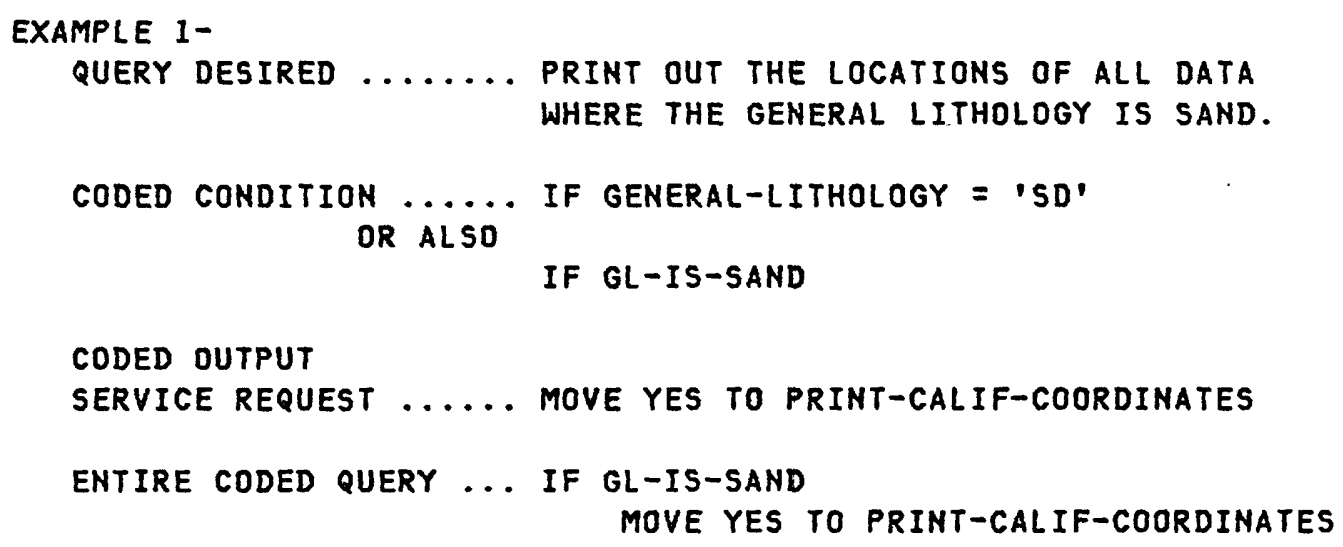

A MORE COMPLEX QUERY MIGHT BE

EXAMPLE 2-

QUERY DESIRED ........ PRINT OUT THE LOCATIONS OF ALL DATA WHERE THE GENERAL LITHOLOGY IS SAND, THE BULK DENSITY IS BETWEEN 90.0 AND $110.0 \mathrm{LB} / \mathrm{CU}$ FT AND THE GROUNDWATER IS LESS THAN 30 FEET.

CODED CONDITION

IF GL-IS-SAND AND BD-VALUE-AYAIL; AND BD-VALUE NOT < 90 AND NOT > 110 AND WT-VALUE-AVAIL AND WT-VALUE $<30$

CODED OUTPUT SERVICE REQUEST ..... MOVE YES TO PRINT-CALIF-COORDINATES

ENTIRE CODED QUERY ... IF GL-IS-SAND AND BD-VALUE-AVAIL AND BD-VALUE NOT < 90 AND NOT > 110 AND WT-VALUE-AVAIL AND WT-VALUE < 30 MOVE YES TO PRINT-CALIF-COORDINATES 
OR

EXAMPLE 3-

QUERY DESIRED ....... PRINT OUT THE LOCATIONS AND DEPTH OF ALL GROUNDWATER MEASUREMENTS AND THE DATE OF MEASUREMENT.

CODED CONDITION ..... IF WT-VALUE-AVAIL

CODED OUTPUT

SERVICE REQUEST ..... MOVE YES TO

PRINT-DEPTH-TO-SAMPLE

PRINT-DATE-OF-MEASUREMENT

PRINT-PARAMETER-VALUE

PRINT-WATER-TABLE

ENTIRE CODED QUERY ... IF WT-VALUE-AVAIL

MOVE YES TO

PRINT-DEPTH-TO-SAMPLE

PRINT-DATE-OF-MEASUREMENT

PRINT-PARAMETER-VALUE

PRINT-WATER-TABLE

IF ANY DATA ITEM BESIDES THE CALIFORNIA COORDINATES IS REQUESTED

TO BE PRINTED THE CALIFORNIA COORDINATES ARE AUTOMATICALLY PRINTED

AS PART OF THE RESPONSE.

AS SHOWN ABOVE, THE "MOVE YES TO" NEED ONLY BE CODED ONCE FOR EACH QUERY FOLLOWED BY A LIST OF THE PRINT SERVICE NAMES DESIRED. THE OUTPUT SERVICE REQUEST DATA NAMES MAY BE CODED IN ANY ORDER.

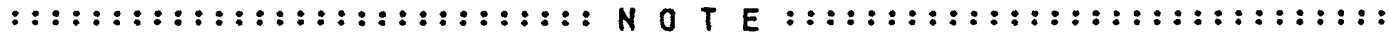

BECAUSE OF THE EXPEDIENCE REQUIRED OF THE DESIGN, A SPECIAL CODING SEQUENCE IS REQUIRED OF ALL QUERIES INVOLVING NUMERIC COMPARISONS. THIS SEQUENCE REQUIRES THAT YOU MUST TEST THE AVAILABILITY OF A VALUE BEFORE YOU TEST THE THE ACTUAL VALUE ITSELF. THIS MUST BE CODED AS SHOWN ABOVE.

FROM THE ABOVE EXAMPLES

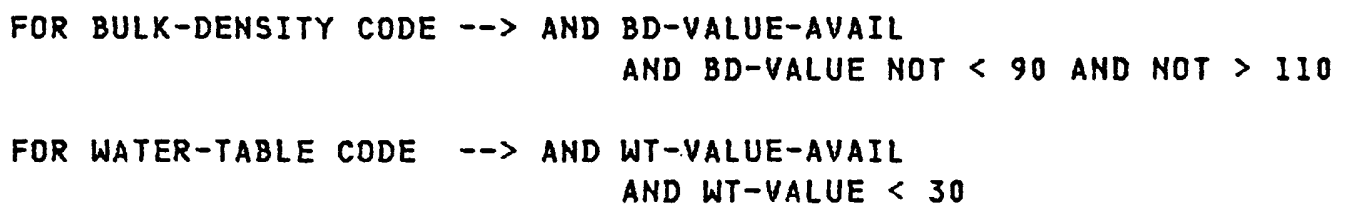

FAILURE TO CODE THIS SEQUENCE WILL RESULT IN THE ABNORMAL TERMINATION OF THE QUERY PROCESSING. 
THE FORMAT DF THE CODED QUERY IS FREE FORM WITHIN CHARACTERS 2 THROUGH 62 OF A QUERY LINE. CHARACTER I IS RESERVED AS A COMMENT INDICATOR. A COMMENT MAY BE INSERTED IN YOUR QUERY BY ENTERING AN ASTERISK IN CHARACTER 1. FOR NON-COMMENT LINES CHARACTER I MUST BE BLANK. BLANK LINES MAY BE USED TO IMPROVE THE READABILITY OF THE CODED QUERY.

YOUR QUERY CONDITION MAY BE MADE AS COMPLEX AS NEEDED BY USING "AND'S" AND "OR'S". THE GROUPING FOR COMPARISON PURPOSES MUST BE CONTROLLED THROUGH THE USE OF PARENTHESES. THE RULES FOR GROUPING FOLLOW CONYENTIONAL SET LOGIC RULES FOR EVALUATION OF THE "AND'S" AND "OR'S".

POLYGON SEARCHING MAY BE SPECIFIED BY AS FOLLOWS:

MOVE 'A BBBBBBBE CCCCCCCN' TO CALIF-COORDINATE-POINT (01)

MOVE 'A BBBBBBBE CCCCCCCN' TO CALIF-COORDINATE-POINT (24)

WHERE "A" IS THE ZONE VALUE OF THE GALIFORNIA COORDINATES.

"B" IS THE EAST VALUE OF THE CALIFORNIA COORDINATES.

"C" IS THE NORTH VALUE OF THE CALIFORNIA COORDINATES.

THE "E", "N" AND BLANKS ARE REQUiREd AS PART OF VALUE.

THE MAXIMUM NUMBER OF POINTS CURRENTLY ACCEPTED BY THE PROGRAŸ IS 24.

WHEN A POLYGON SEARCH IS SPECIFIED THE FOLLOWING MUST BE CODED AS

PART OF THE CONDITION PORTION OF THE QUERY:

"A-SAMPLE-IS-IN-THE-POLYGON"

EXAMPLE 4-

MOVE '6 $1480000 E$ 0539000N' TO CALIF-COORDINATE-POINT (1)

MOVE '6 $1480000 E$ 0541000N' TO CALIF-COORDINATE-POINT (2)

MOVE '6 1515000E 0623000N' TO CALIF-COORDINATE-POINT (3)

MOVE '6 1515000E 0625000N' TO CALIF-COORDINATE-POINT (4)

MOVE ' 1 ' TO RELEASE-AUTHORITY-AUTHORIZED

IF A-SAMPLE-IS-IN-THE-POLYGON

MOVE YES TO PRINT-GENERAL-LITHOLOGY

PRINT-WATER-TABLE

PRINT-BULK-DENSITY

PRINT-S-WAVE-VELOCITY

PRINT-STND-PENETRATION-TEST

PRINT-SEISMIC-IMPEDANCE

PRINT-ALL-SAMPLE-DATA-ITEMS

A MORE DETAILED EXPLANATION OF THE CODING OF A QUERY IS PROVIDED

WITHIN THE MENUS THEMSELVES. THE READER IS REFERRED TO THE

ATTACHMENTS. 


\section{INTERNAL/TECHNICAL VIEW}

PROGRAM DESIGN CONCEPT:

THE PROBLEM WHICH THE DESIGN OF THE DATA RETRIEVAL SOLVES IS HOW TO PROVIDE AD HOC DATA RETRIEVAL CAPABILITY WITH THE RESTRICTION OF SIX TO EIGHT MONTHS DEVELOPMENT TIME FOR THE ENTIRE APPLICATION USING ONLY ONE PERSON DURING THE DEVELOPMENT. THE SIX TO EIGHT MONTHS WAS NOT SUFFICENT TIME TO DO AN ADEQUATE EVALUATION, SELECTION AND INSTALLATION OF A DATA BASE MANAGEMENT SYSTEM, AND THUS WAS DISCARDED AS A VIABLE ALTERNATIVE. THIS TIME FRAME WAS ALSO NOT SUFFICIENT TO DEVELOP AND IMPLEMENT A NEW QUERY GRAMMER AND REPORTING CAPABILITY TO PROCESS THE DATA STRUCTURE DESIGNED. A MARKET SURVEY ALSO PROVED FRUITLESS.

THE QUESTION WAS THEN ASKED, "WHAT DO I HAVE THAT IS AVAILABLE, WELL TESTED, INSTALLED AND OPERATIONAL WHICH PROVIDES SYNTATIC AND SEMANTIC ANALYSIS OF "IF' TYPE STATEMENTS?" THE ANSWER WAS: "ANY OF SEVERAL COMPILERS." THE DECISION WAS MADE TO USE COBOL BECAUSE OF ITS "IF" CAPABILITIES, THE USE OF CONDITION NAMES ("88-LEVELS"), THE ABILITY TO CREATE PSEUDO ENGLISH STATEMENTS USING ITS SYNTAX AND THE PORTABILITY OF THE RESULTING SOURCE CODE.

THE RESULTING QUERY LANGUAGE CONSISTS OF COBOL "IF" STATEMENTS AND "MOVE" STATEMENTS. THE USER IS TAUGHT ONLY TO CODE THE COBOL "IF" STATEMENT AND SIMPLE "MOVE" STATEMENTS. THE USER NEVER SEES THE CONTEXT IN WHICH THE CODED QUERY IS PLACED AND IS NOT TROUBLED, OVERWHELMED OR CONFUSED WITH THE DETAILS OF THE CONTEXT. IN CONCEPT THE USER SAYS "IF A SAMPLE MEETS THE CONDITION CODED, SHOW ME THE VALUES OF SOME SET OF DATA ITEMS."

AS IMPLEMENTED IN THE DESIGN THE USER-CODED QUERY IS REFORMATTED, USING A UTILITY PROGRAM, TO CONFORM TO THE REQUIREMENTS OF COLUMN 7 AND MARGIN "B" OF COBOL. THIS IS COMBINED WITH A FEW OTHER PROCEDURE STATEMENTS AND COMPILED. THE RESULTING LOAD MODULE IS DYNAMICALLY CALLED, AND ALL THE DATA VALUES FOR EACH SAMPLE FROM THE STORAGE FILE ARE PASSED TO IT FOR TESTING. THE OUTPUT OF THE QUERY LOAD MODULE IS SIMPLY THE ANSWER TO THE QUESTION "DOES THIS SAMPLE SATISFY THE CONDITIONS OF YOUR QUERY? YES OR NO?" IF YES, THE PROGRAM PROCESSED THE OUTPUT SERVICES CODED. IF NO, THE PROGRAM OBTAINS THE NEXT SAMPLE. 
THE DATA RETRIEVAL FUNCTION IS DESIGNED AS FOLLOWS:

- DURING START UP PROCESSING

A) CALL THE USER QUERY TO CHECX THE QUERY CONTROL SERVICES REQUESTED AND SET UP ANY POLYGON SEARCH REQUESTED.

B) WRITE THE RELEASE AUTHORITY COVER PAGES.

C) WRITE THE USER-CODED QUERY REPORT.

D) REFORMAT AND WRITE ANY ERRORS RESULTING FROM THE COMPILE OF THE USER QUERY.

IF NO ERRORS WERE DETECTED DURING START UP PROCESSING THE FOLLOWING IS DONE FOR EACH SAMPLE FROM THE STORAGE FILE.

- OBTAIN ALL STORAGE SEgMENTS WHICH APPLY TO A SAMPLE. THIS INCLUDES THE SURFACE AND LAYER SEGMENTS.

- if a sample was obtaINed, CyCle all combinations of the SAMPLE DATA AGAINST THE USER QUERY.

- IF THE SAMPLE SATISFIED THE USER QUERY, PROCESS THE OUTPUT SERVICES REQUESTED.

- UPON FINDING THE FIRST SAMPLE WHICH SATISFIES THE QUERY, THE USER'S OUTPUT SERVICE REQUEST IS ANALYZED TO DETERMINE THE INTERNAL PROCESSING REQUIREMENTS. CERTAIN ERRORS MAY BE DETECTED DURING THIS ANALYSIS WHICH WILL. PREVENT FURTHER PROCESSING.

THE FORMAT FOR THE RETRIEVAL REPORT IS DIVIDED INTO "REPORT BLOCKS" WHICH ARE PERFORMED ON AN AS NEEDED BASIS. THE MAIN REPORT BLOCKS LOGICALLY ASSOCIATE WITH THE SEGMENT TYPES. OTHER REPORT BLOCKS BUILD AND WRITE AREAS LIKE THE REPORT TITLES AND HEADINGS.

PROGRAM DESIGN METHODOLOGY:

STRUCTURED PROGRAM DESIGN AS OFFERED BY KEN ORR AND ASSOCIATES, INC. OF TOPEKA, KANSAS WAS THE METHODOLOGY USED FOR THE DESIGN OF THE DATA RETRIEVAL PROGRAM.

PROGRAMMING LANGUAGE:

THE DATA RETRIEVAL PROGRAM IS WRITTEN USING IBM COBOL. KNOWN NON-STANDARD COBOL IS USED TO OBTAIN THE COMPILE DATE AND TIME FROM THE "WHEN-COMPILED" DATA NAME. THE DATE AND TIME OF THE START AND END OF THE RUN IS OBTAINED USING THE "DATE" AND "TIME-OF-DAY" DATA NAMES. THE DATA NAME OPTION OF THE "CALL" STATEMENT IS USED TO CHANGE THE SUBROUTINE WHICH IS THE USER QUERY. ALSO USED IS A SUBROUTINE CALLED "DHSORT" WHICH IS AN INTERNAL TABLE SORT. FOR ANY ONE WHO WISHES TO INSTALL AND USE THIS PROGRAM IT IS SUGGESTED THAT AN ATTEMPT BE MADE TO COMPILE THE PROGRAM AND SEE WHAT ERRORS RESULT. 
DEVELOPMENT ENVIRONMENT:

THE DEVELOPMENT ENVIRONMENT USED TO CREATE AND MAINTAIN THE

DATA RETRIEVAL PROGRAM INCLUDE IBM TSO, FULL-SCREEN EDIT AND PANVALET AND THE IBM OS COBOL INTERACTIVE DEBUG, ALSO REFERRED TO AS "IESTCOB".

PROGRAM CHARACTERISTICS:

WITHOUT THE CODE SEGMENTS OBTAINED USING PANVALET INCLUDE STATEMENTS, THE SOURCE PROGRAM CONTAINS ABOUT 10,000 CARD IMAGES. THE SOURCE PROGRAM AS EXPANDED BY PANVALET CONTAINS ABOUT 16,200 CARD IMAGES.

WORKING STORAGE IS ORGANIZED FUNCTIONALLY ALONG THE LINES OF THE PROCESSING FLOW. THE FIRST ENTRY OF MOST TABLES IS A WORK ENTRY WITH THE REMAINING ENTRIES BEING STORAGE ENTRIES. MOST LITERALS WHICH MIGHT APPEAR IN THE PROCEDURE DIVISION ARE DEFINED IN WORKING STORAGE FOR CROSS REFERENCE PURPOSES.

EACH PARAGRAPH NAME IS SUFFIXED WITH A HYPHEN AND A THREE CHARACTER NUMBER. THIS NUMBER SERVES AS A "VICINITY LOCATOR". IN THOSE STATEMENTS WHICH REFERENCE THE PARAGRAPH NAME (E.G. PERFORM), THIS NUMBER POINTS TO THE VICINITY IN THE COBOL SEQENCING (COLUMNS I THRU 6) WHERE THE PARAGRAPH NAME IS DEFINED. THIS NUMBER IS THE FIRST THREE NUMERIC CHARACTERS OF THE COBOL SEQUENCE NUMBER OF THE LINE ON WHICH THE PARAGRAPH NAME IS DEFINED.

DATA FILE CHARACTERISTICS:

QUERY ..... THE INPUT QUERY TEXT FILE CONTAINS THE TEXT OF THE USER-CODED QUERY. THE QUERY TEXT FILE RECORDS ARE 80 CHARACTERS IN LENGTH. THE SECOND QUERY INPUT FILE CONTAINS THE MESSAGES FROM THE COBOL COMPILER. FOR THOSE NON-IBM INSTALLATIONS THE PROGRAM WILL REQUIRE MODIFICATION TO ADAPT THIS PORTION OF THE TO THE REQUIREMENTS OF THE NON-IBM COBOL COMPILER.

STORAGE ... THE STORAGE FILE IS A SEQUENTIAL FILE AND CONTAINS THE DATA SEARCHED BY THE QUERY PROCESS. THE STORAGE SEGMENTS ARE 96 CHARACTERS IN LENGTH.

REPORT ... THERE ARE THREE REPORT FILES GENERATED.

1) THE STRATIGRAPHIC PROPERTIES AND DATA REPORT.

2) THE USER RUN PROFILE WHICH PROVIDES STATISTICAL INFORMATION TO THE USER.

3) THE RUN CONTROLS REPORT WHICH PROVIDES STATISTICAL INFORMATION TO THE SYSTEM PERSONNEL WHICH MAKE THE PROCESSING RUNS.

ALL REPORT FILE PRINT LINES ARE 133 CHARACTERS IN LENGTH WITH THE FIRST CHARACTER BEING A CARRIAGE CONTROL CHARACTER. 
SUPPORT ... THE PROGRAM REQUIRES TWO SUPPORT FILES.

1) THE APPLICATION SUPPORT INDEX.

THIS FILE SERVES AS AN INDEX FOR THE SECOND SUPPORT FILE.

2) THE DATA SOURCE SUPPORT FILE.

THIS FILE SERVES AS THE SOURCE FOR THE LITERAL

DISPLAYED IF THE PRIMARY DATA SOURCE IS REQUESTED.

TO OBTAIN THE CORRECT DATA SOURCE LITERALS FOR

THIS APPLICATION THE APPLICATION SUPPORT INDEX

MUST BE USED. THIS FILE IS UNIQUE TO EACH

INSTALLATION. IT IS REQUIRED TO PROCESS ANY

QUERIES AND MUST BE CREATED PRIOR TO ANY QUERY RUNS.

A METHOD TO CREATE THE APPLICATION SUPPORT INDEX AND DATA SOURCE SUPPORT FILES IS PROVIDED WITH A REQUEST FOR THE PROGRAM SOURCE CODE. THE APPLICATION MESSAGE FILE FOR THIS PROGRAM IS ALSO PROVIDED.

TESTING: -

TESTING THE DATA RETRIEVAL CAPABILITY CONSISTS OF CODING A SET OF QUERIES WHICH EXERCISE VARIOUS OPTIONS AGAINST A STORAGE FILE CONTAINING VARIOUS COMBINATIONS OF SEGMENTS AND VALUES. BECAUSE OF THE AD HOC NATURE OF THE RETRIEVAL CAPABILITY, TESTING CONSISTS OF TRYING AS MANY QUERIES AS IS REASONABLE, BASED ON SOME SCHEME WHICH SAMPLES A REPRESENTATIVE CROSS-SECTION OF ALL POSSIBLE QUERIES. THESE TEST QUERIES ARE RUN AGAINST A TEST STORAGE FILE, WHICH MAY CONTAIN VERY ARIFICIAL DATA COMBINATIONS, BUT WHICH MUST CONTAIN A REPRESENTATIVE CROSS-SECTION OF ALL POSSIBLE SEGMENT AND DATA VALUE COMBINATIONS. 
IN THE SYSTEM AS IMPLEMENTED, THE USER HAS ACCESS TO DATA FROM A VARIETY OF SOURCES, SOME OF WHICH ARE CONFIDENTIAL. IN ORDER TO SAFEGUARD ANY STORED DATA FROM UNAUTHORIZED ACCESS A TWO-LEVEL SECURITY SYSTEM HAS BEEN PROVIDED. THE FIRST LEVEL IS A RELEASE AUTHORITY MECHANISM. THE SECOND LEVEL IS ENCRYPTION OF THE PHYSICAL DATA.

\section{RELEASE AUTHORITY}

AS DATA IS ENTERED INTO THE SYSTEM IT MUST INCLUDE, EXPLICITLY OR IMPLICITLY, A RELEASE AUTHORITY. THIS RELEASE AUTHORITY IS STRUCTURED AS FOLLOWS:

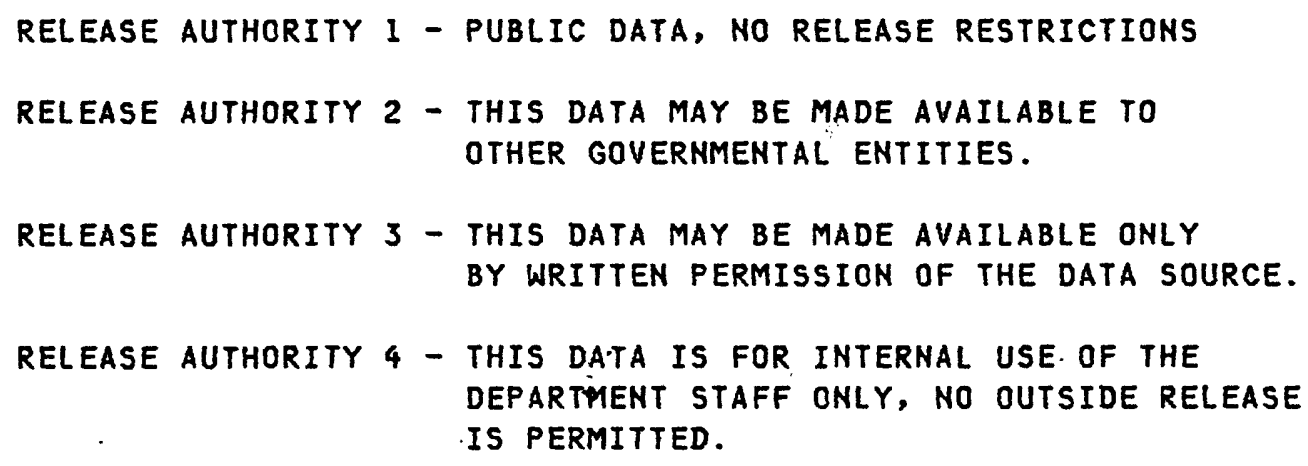

THE RELEASE AUTHORITY IS IMPLICIT IN THE DATA SOURCE CODE UNLESS EXPLICITLY CODED AS PART OF THE PARAMETER DATA ITEM VALUES ENTERED ON THE USER-CODED INPUT FORM.

A QUERY PROCESSED AGAINST THE STORAGE FILE MUST SPECIFY A REQUESTED RELEASE AUTHORITY. ANY DATA ITEMS ON THE FILE WHOSE RELEASE AUTHORITY EXCEEDS THE REQUESTED AUTHORITY ARE WITHHELD FROM USER VIEW. ADDITIONALLY, OUTPUT REPORTS CONTAIN COVER SHEETS WHICH INDICATE THE RELEASE AUTHORITY OF THE REPORTED DATA AND CLERICAL INSTRUCTIONS APPROPRIATE TO THE REQUESTED RELEASE AUTHORITY.

\section{DATA ENCRYPTION}

THE PHYSICAL DATA IS ENCRYPTED TO AVOID THE POSSIBILITY THAT PRINTING OR DUMPING OF THE PHYSICAL DATA COULD DIVULGE ANY INFORMATION OF USE TO AN UNAUTHORIZED RECIPIENT. COPIES OF THE ENCRYPTION ROUTINES OR ALGORITHMS ARE NOT AVAILABLE. DISCUSSIONS RELATING TO THE ENCRYPTION METHODOLOGY WILL NOT BE ENTERTAINED. THE SYSTEM WILL FUNCTION WITHOUT THE ENCRYPTION FEATURE. WE RECOMMEND ANYONE INTERESTED IN, OR REQUIRING THIS FUNCTION, TO EITHER USE ONE OF THE COMMERCIALLY AVAILABLE PACKAGES OR WRITE THEIR OWN, AS THEY PREFER. 


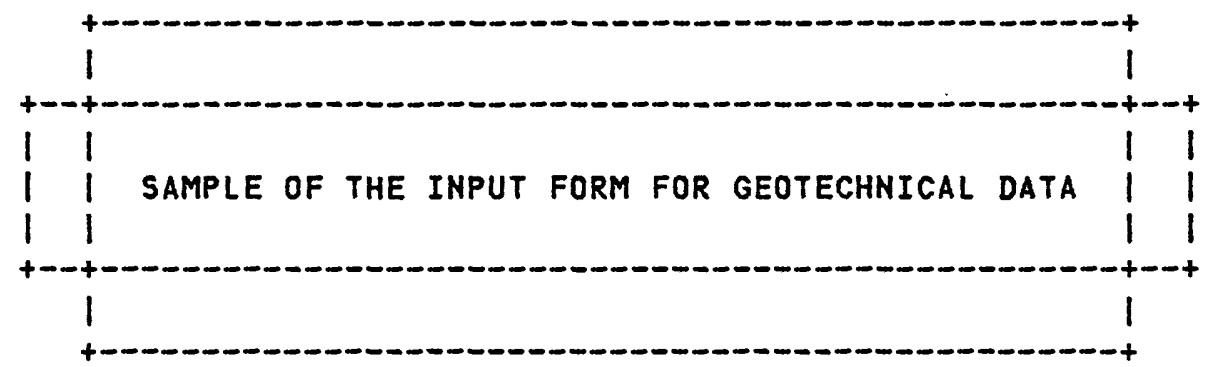


INPUT FORM FOR GEOTECHNICAL DATA

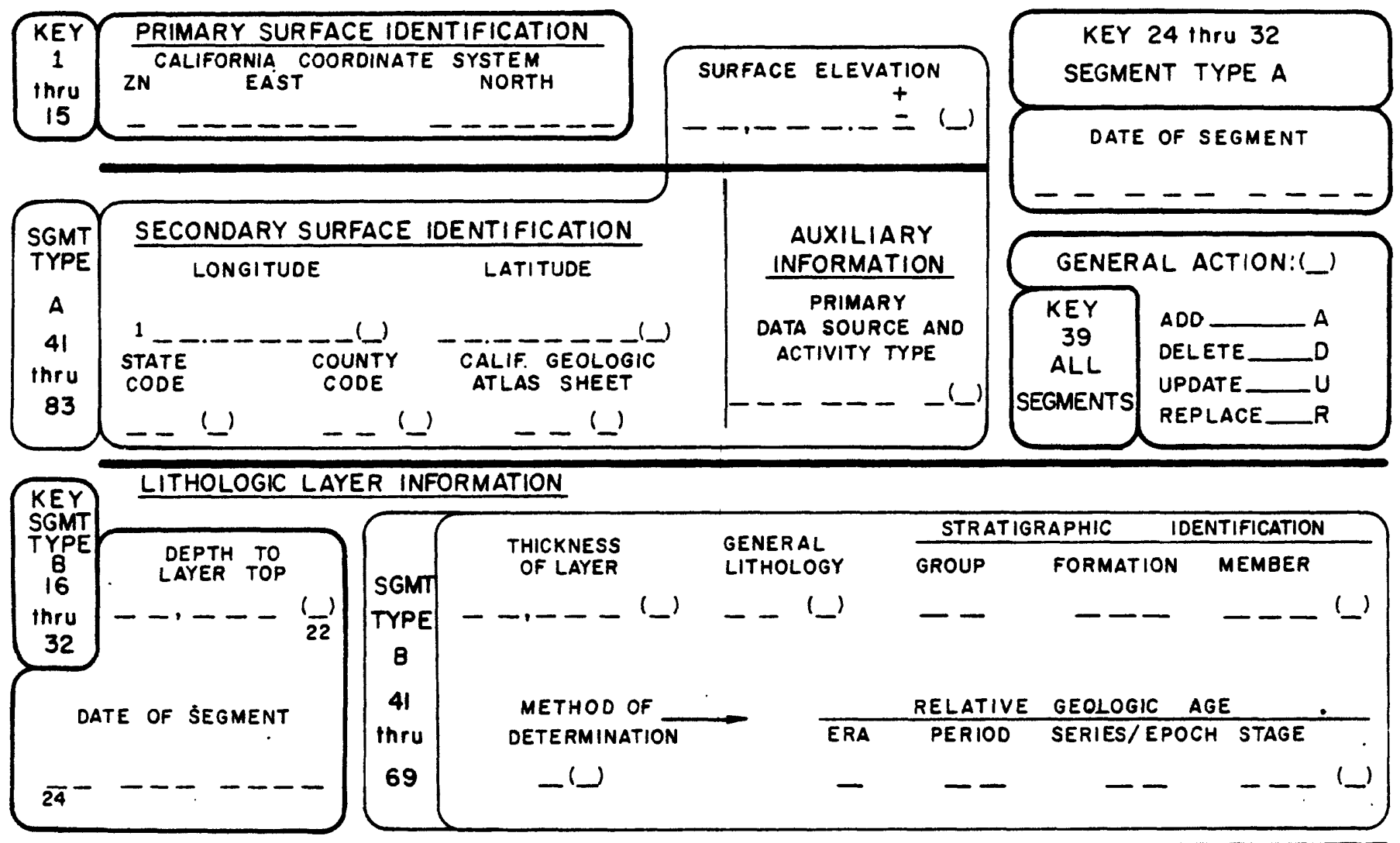

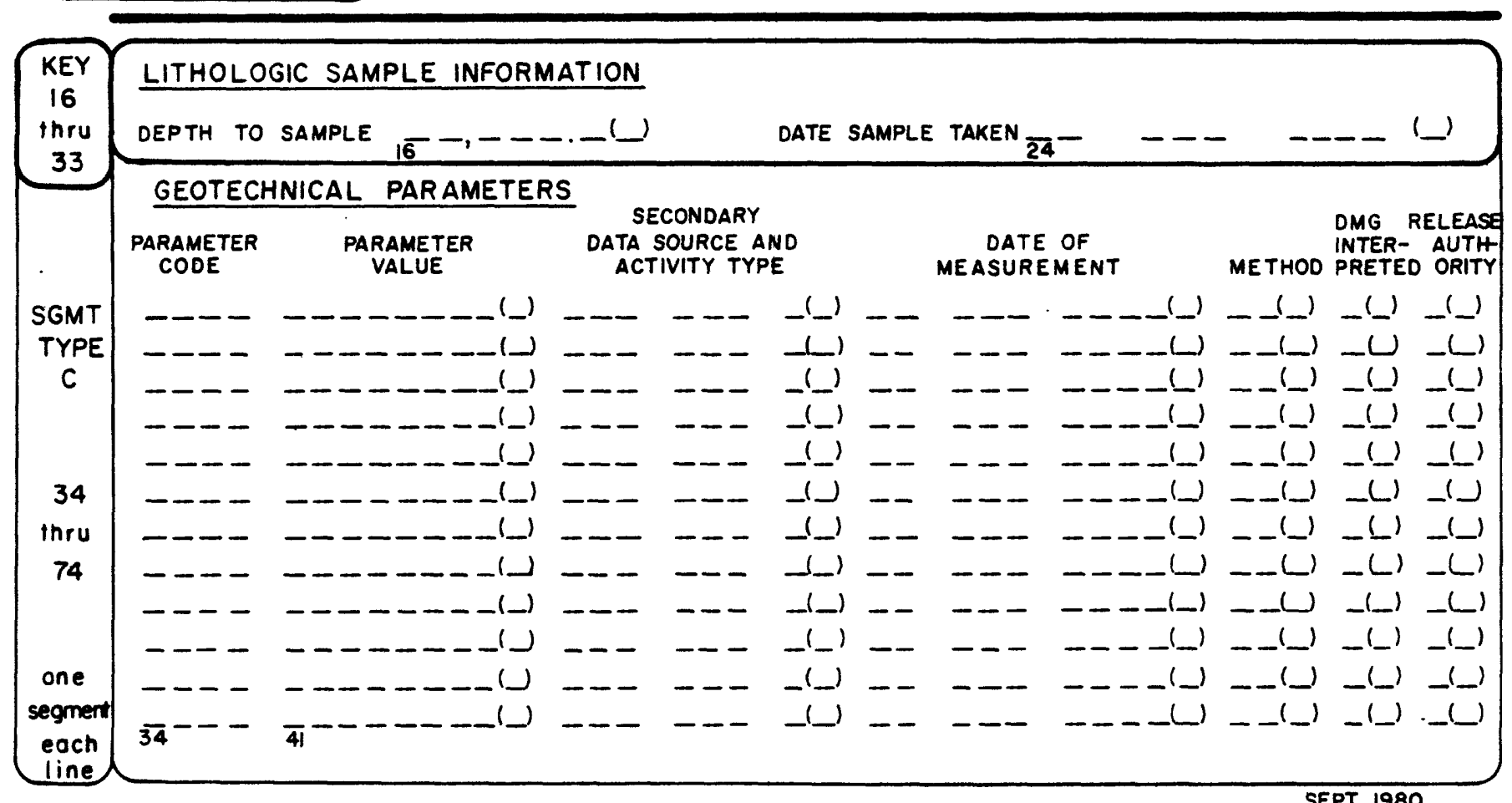

EDP-4 


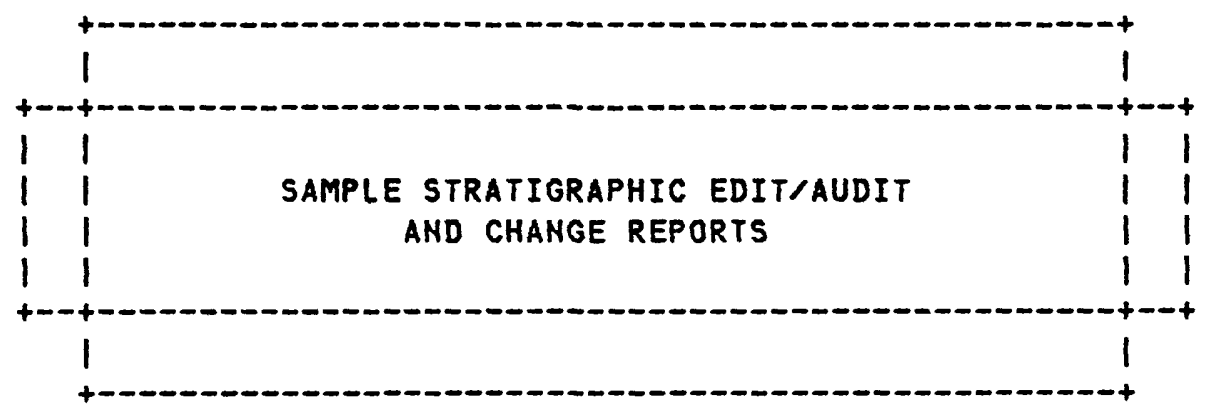

THE SAMPLE STRATIGRAPHIC EDIT/AUDIT AND CHANGE REPORTS PROVIDED AS THIS ATTACHMENT GENERATE A SMALL STORAGE FILE. THIS FILE IS USED AS THE STORAGE FILE FOR THE SAMPLE QUERIES AND THE RESULTING STRATIGRAPHIC PROPERTIES AND DATA REPORTS PROVIDED AS ATTACHMENT $G$. 


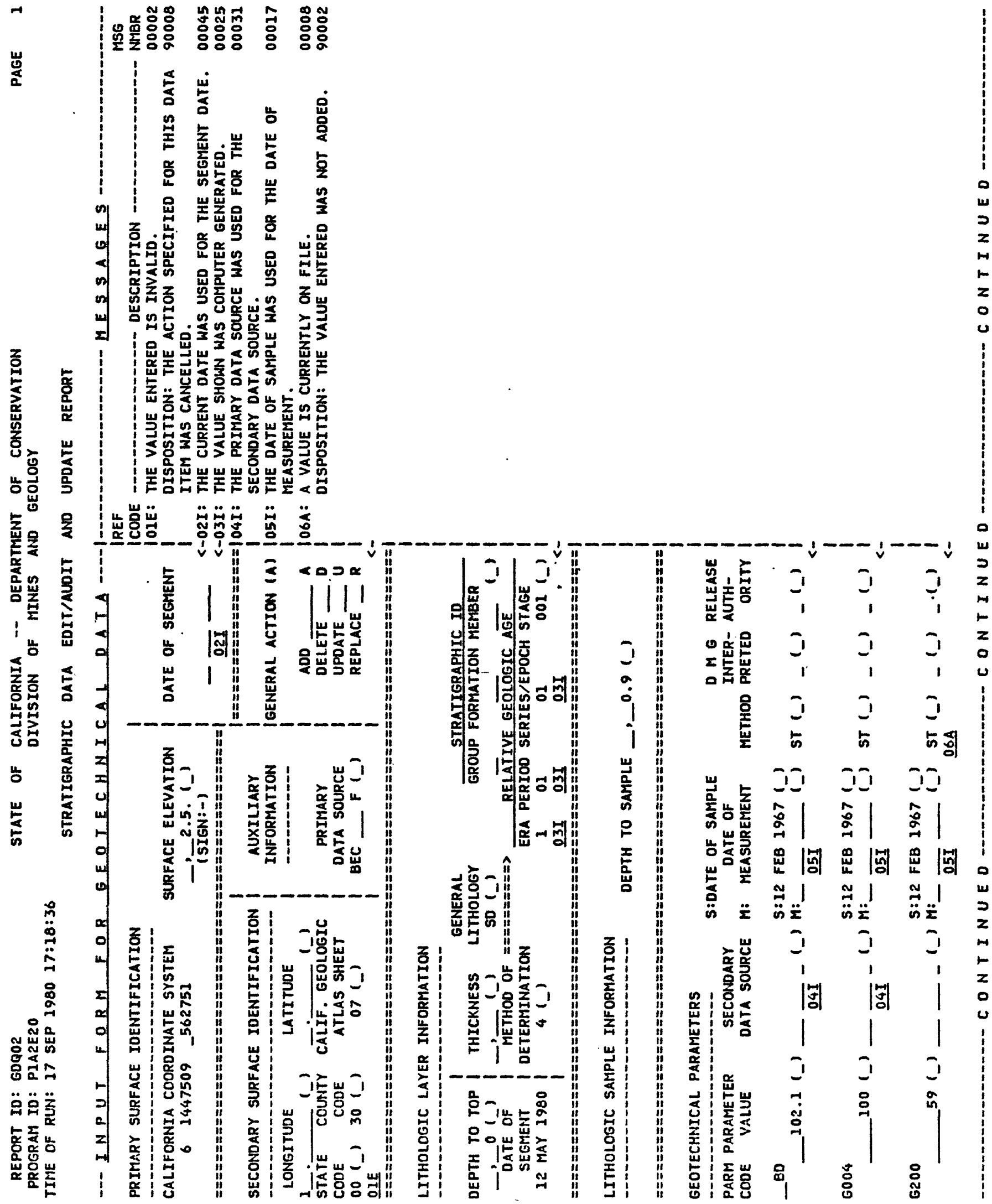



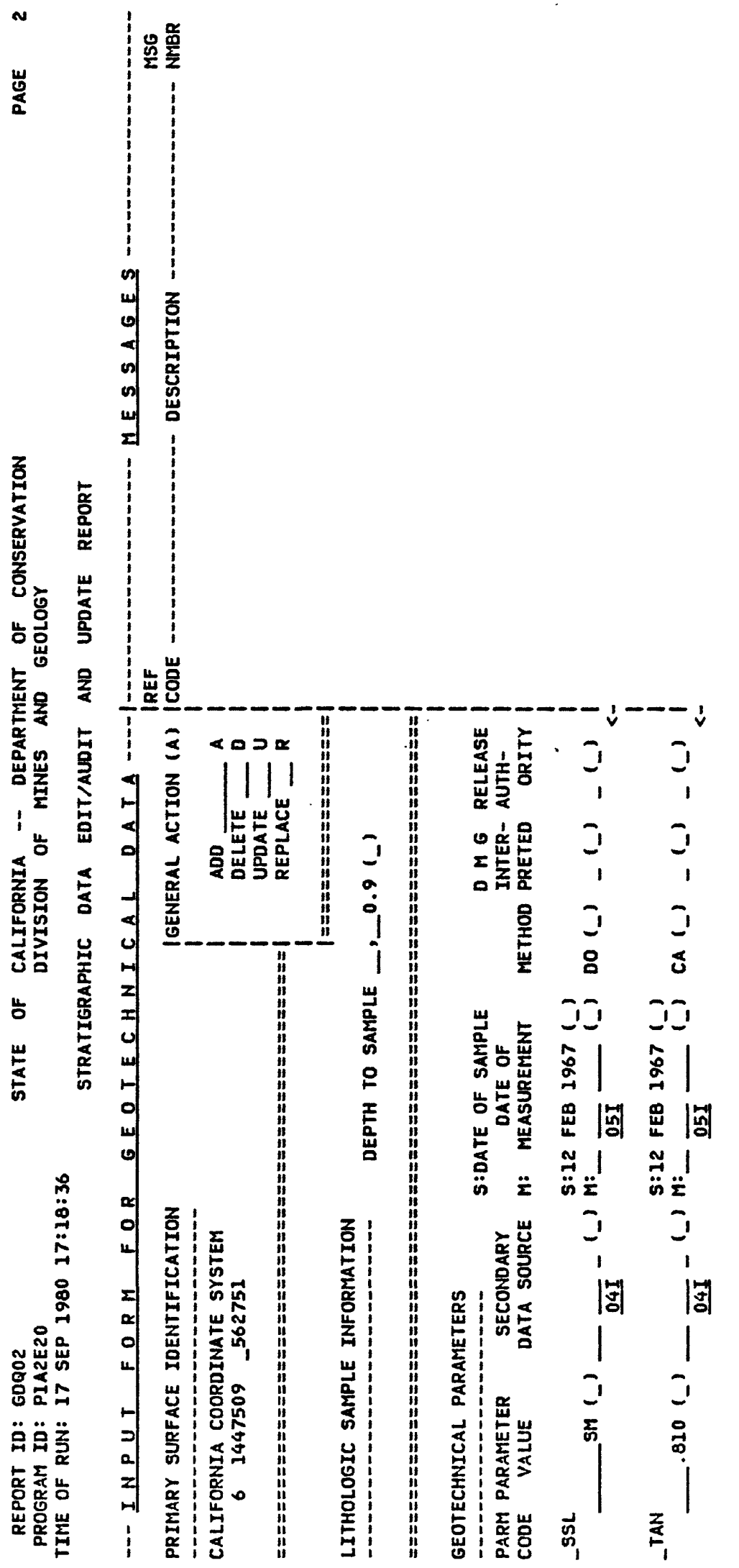


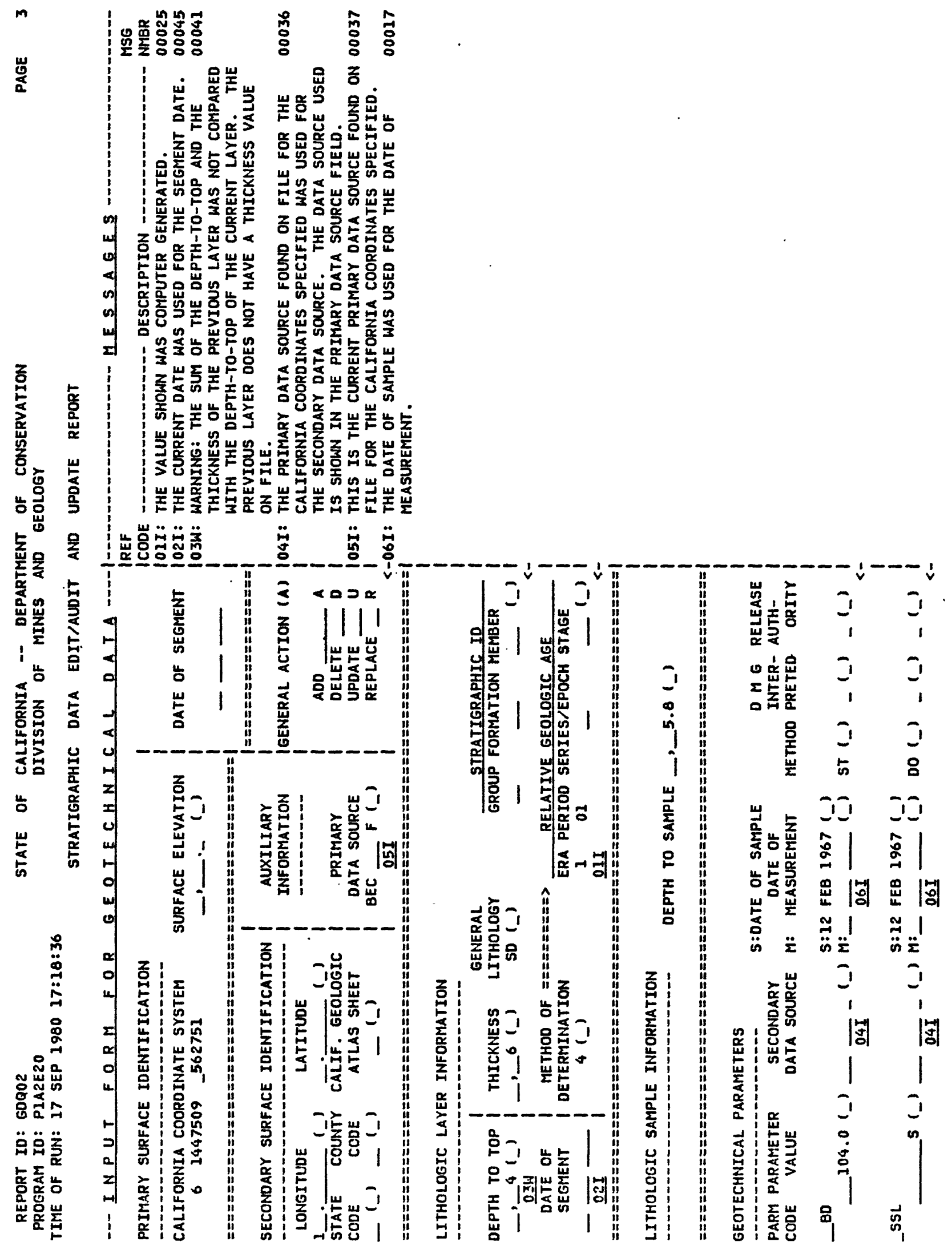




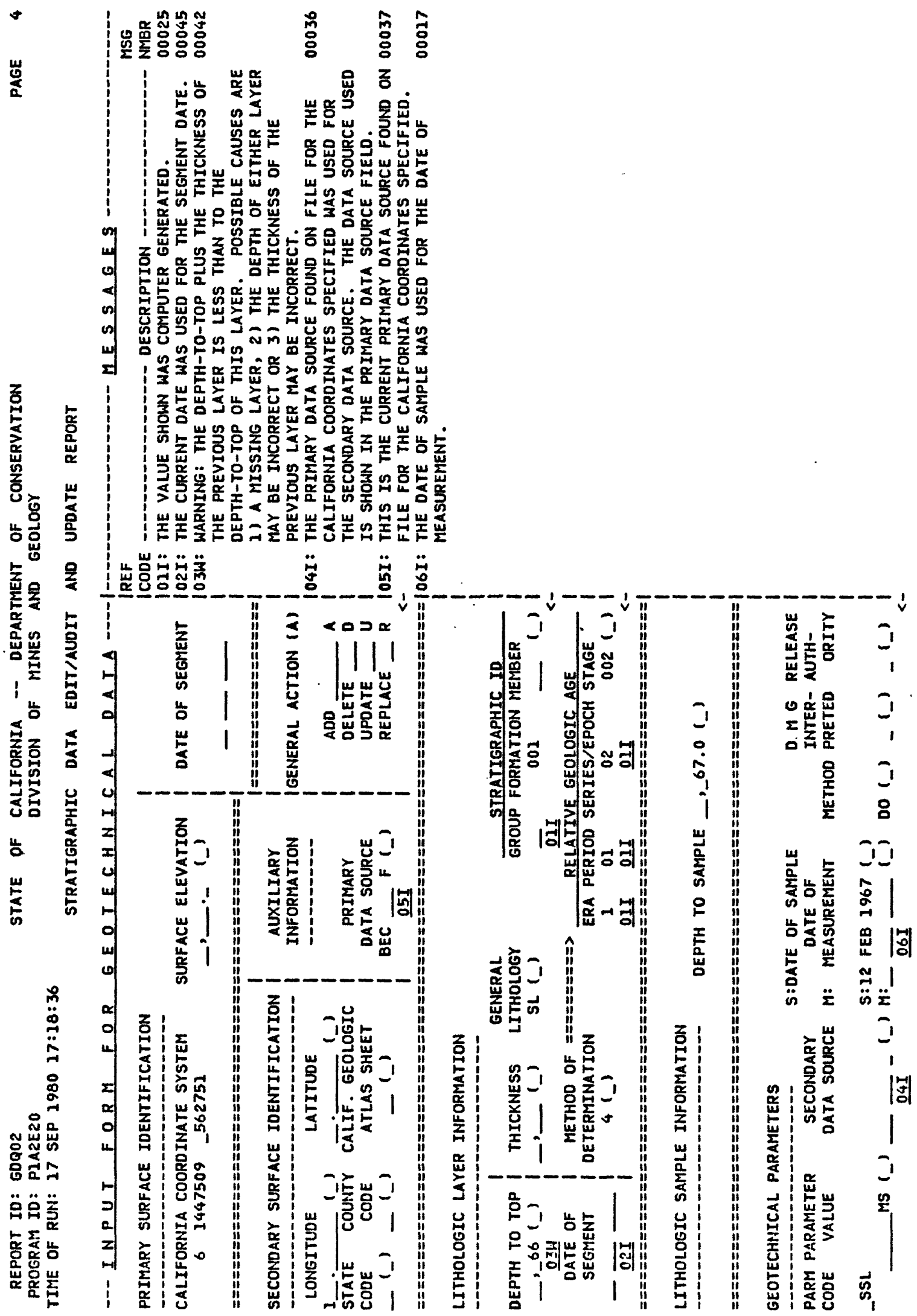




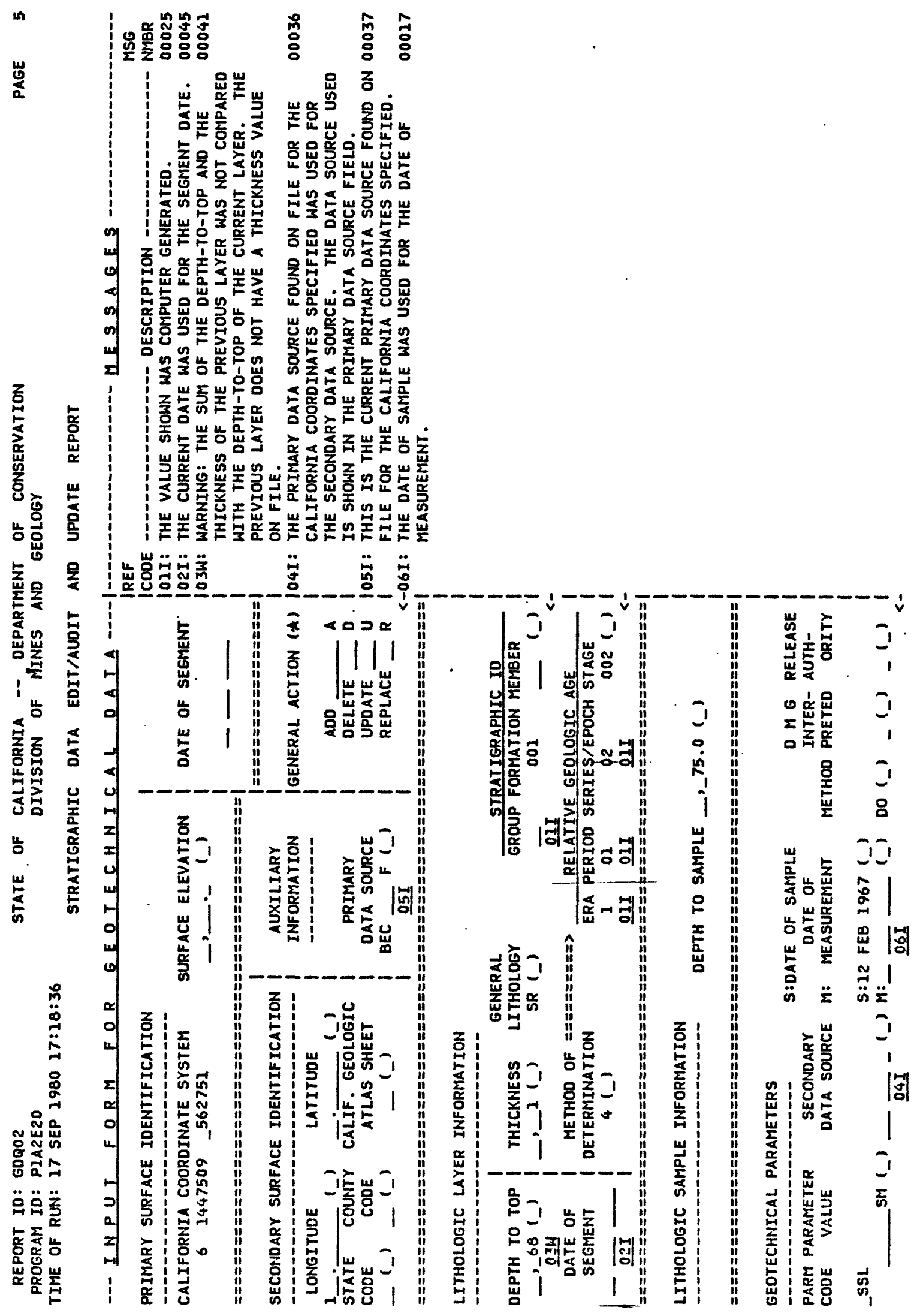




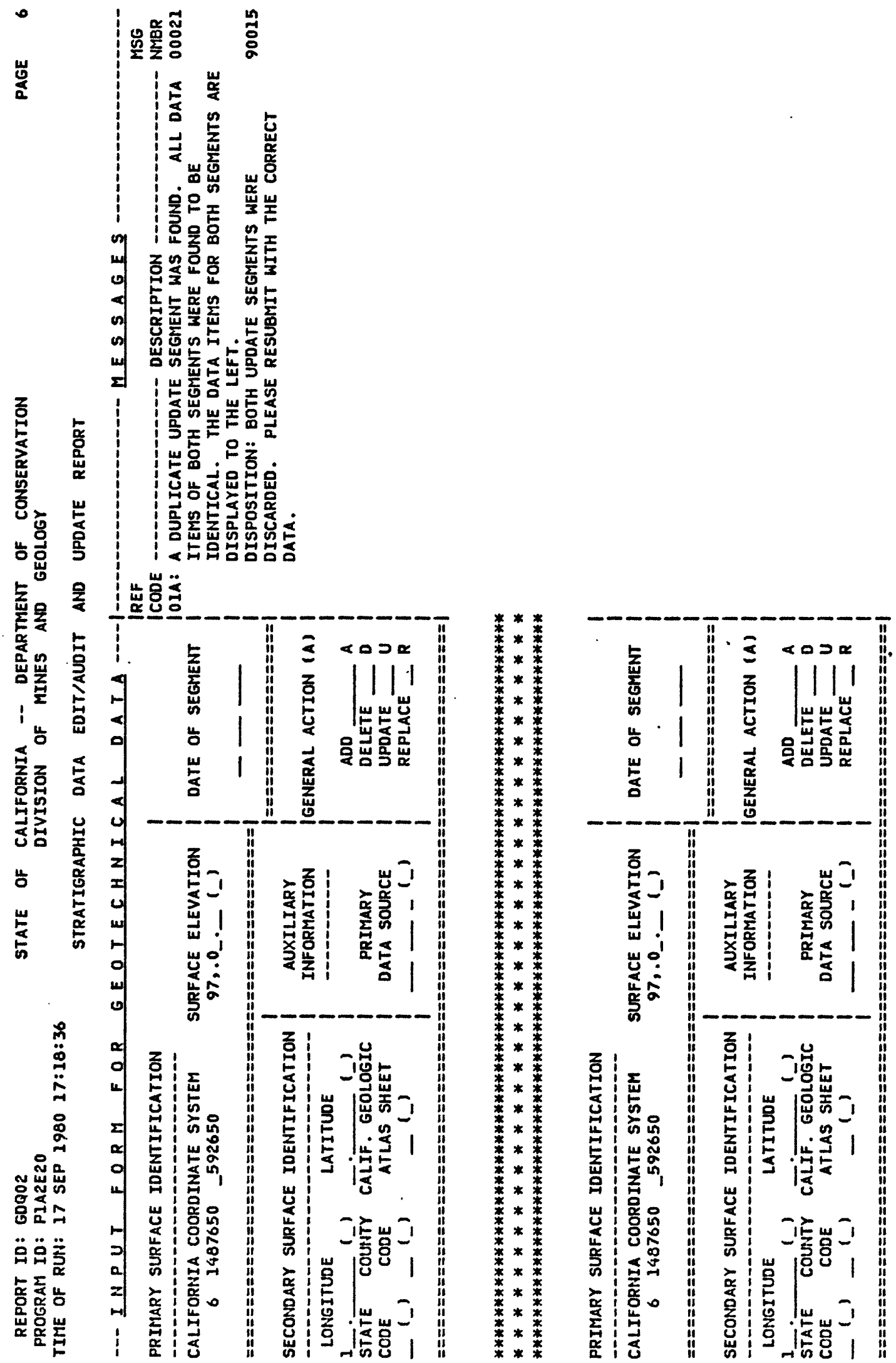




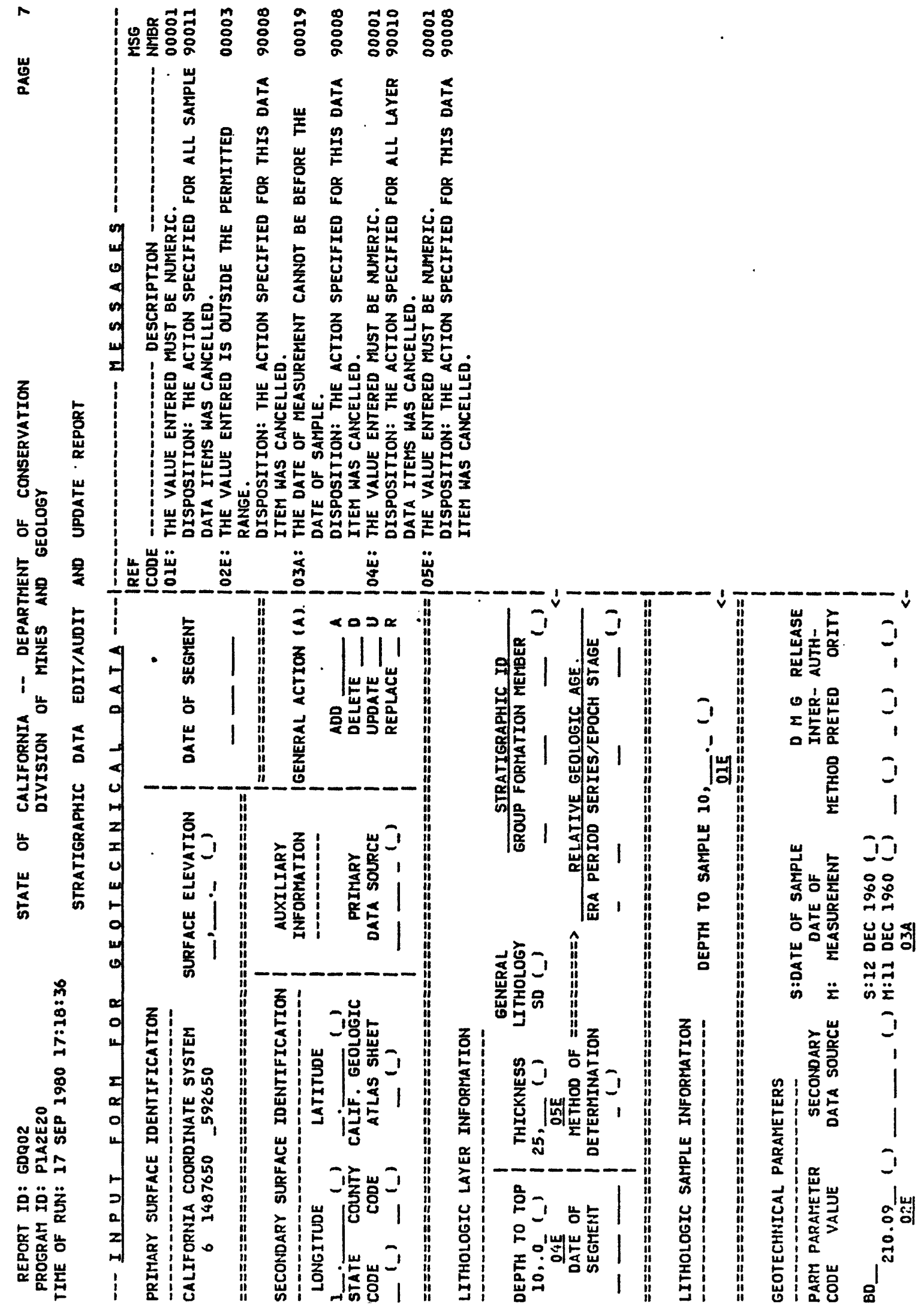



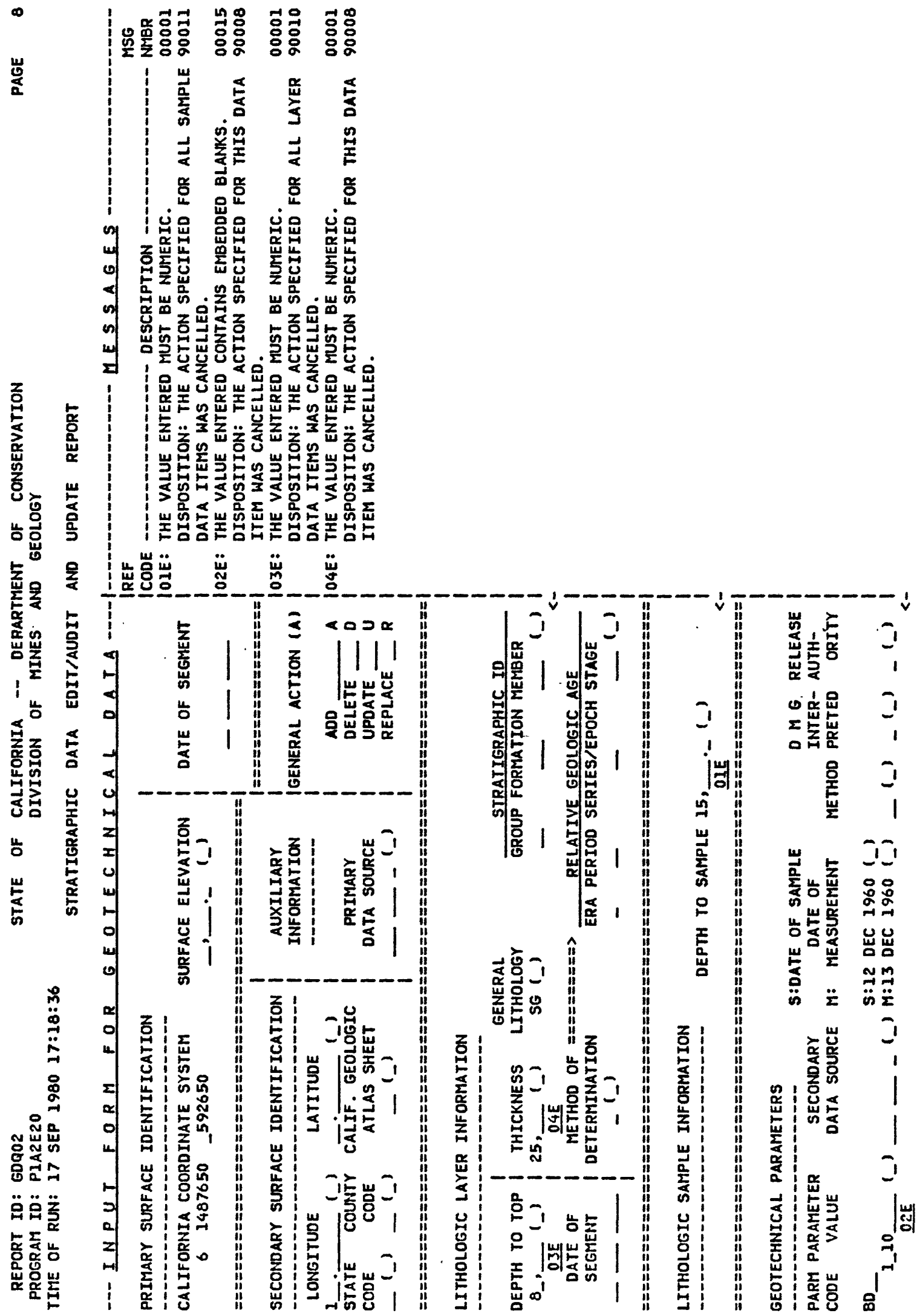


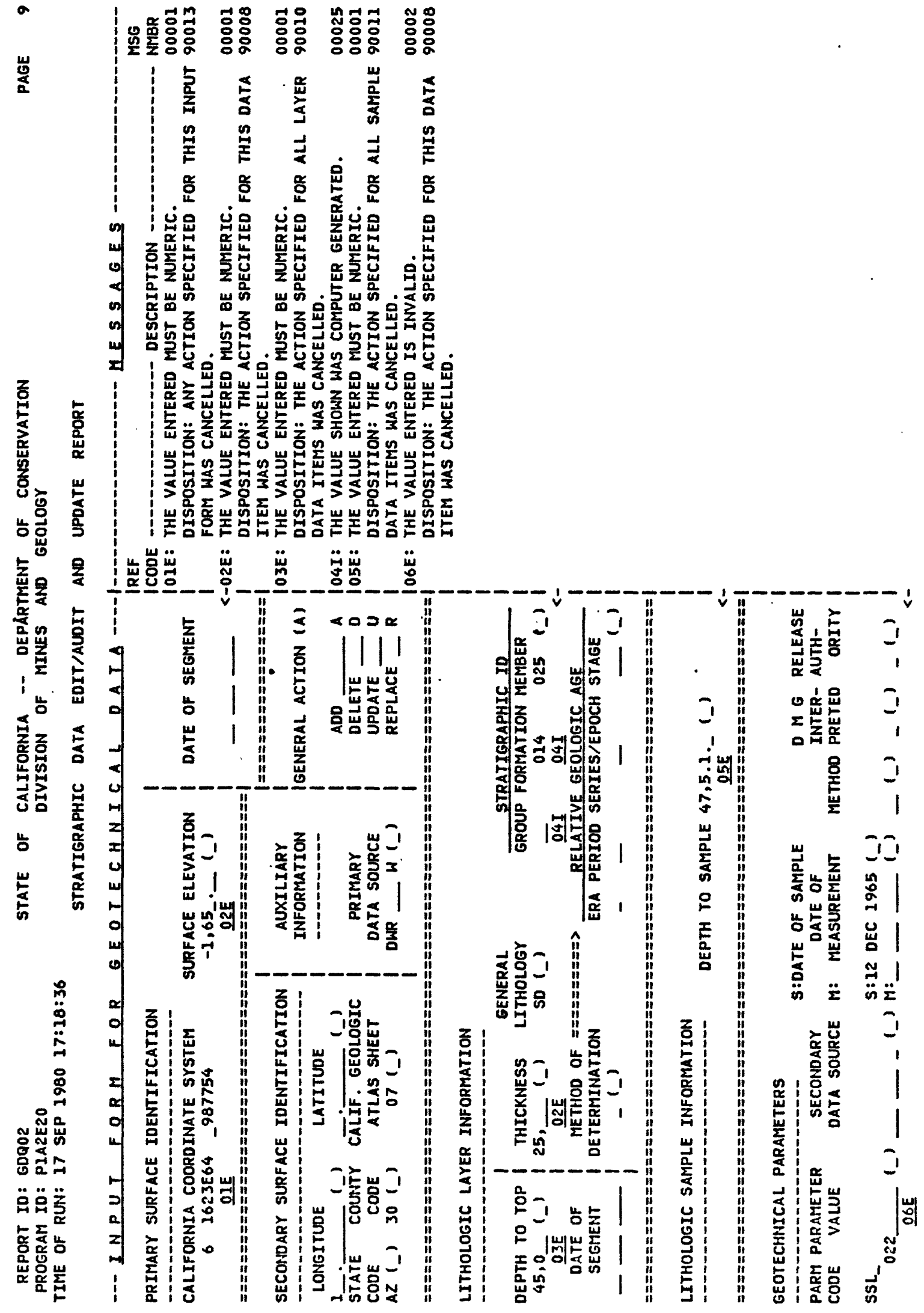




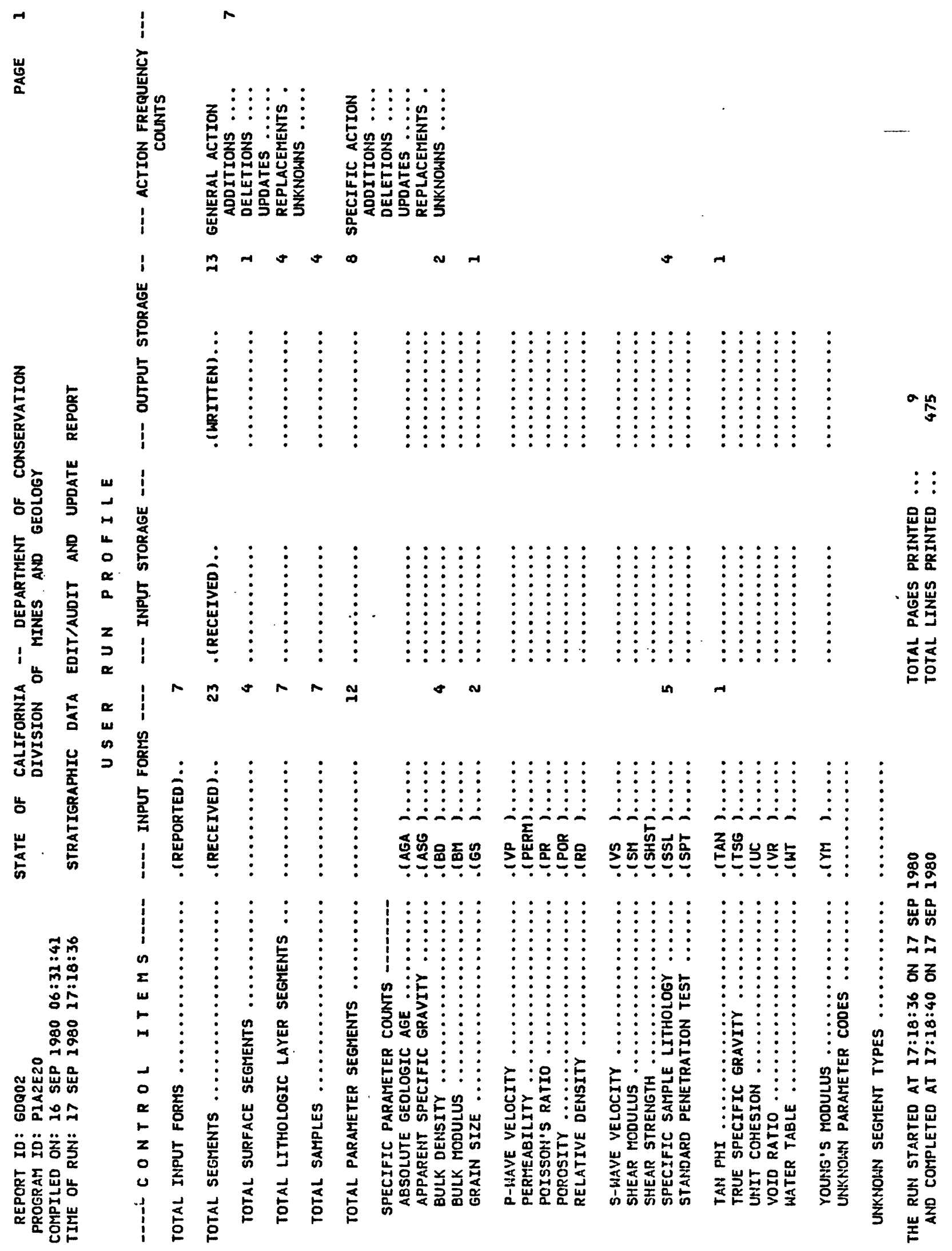




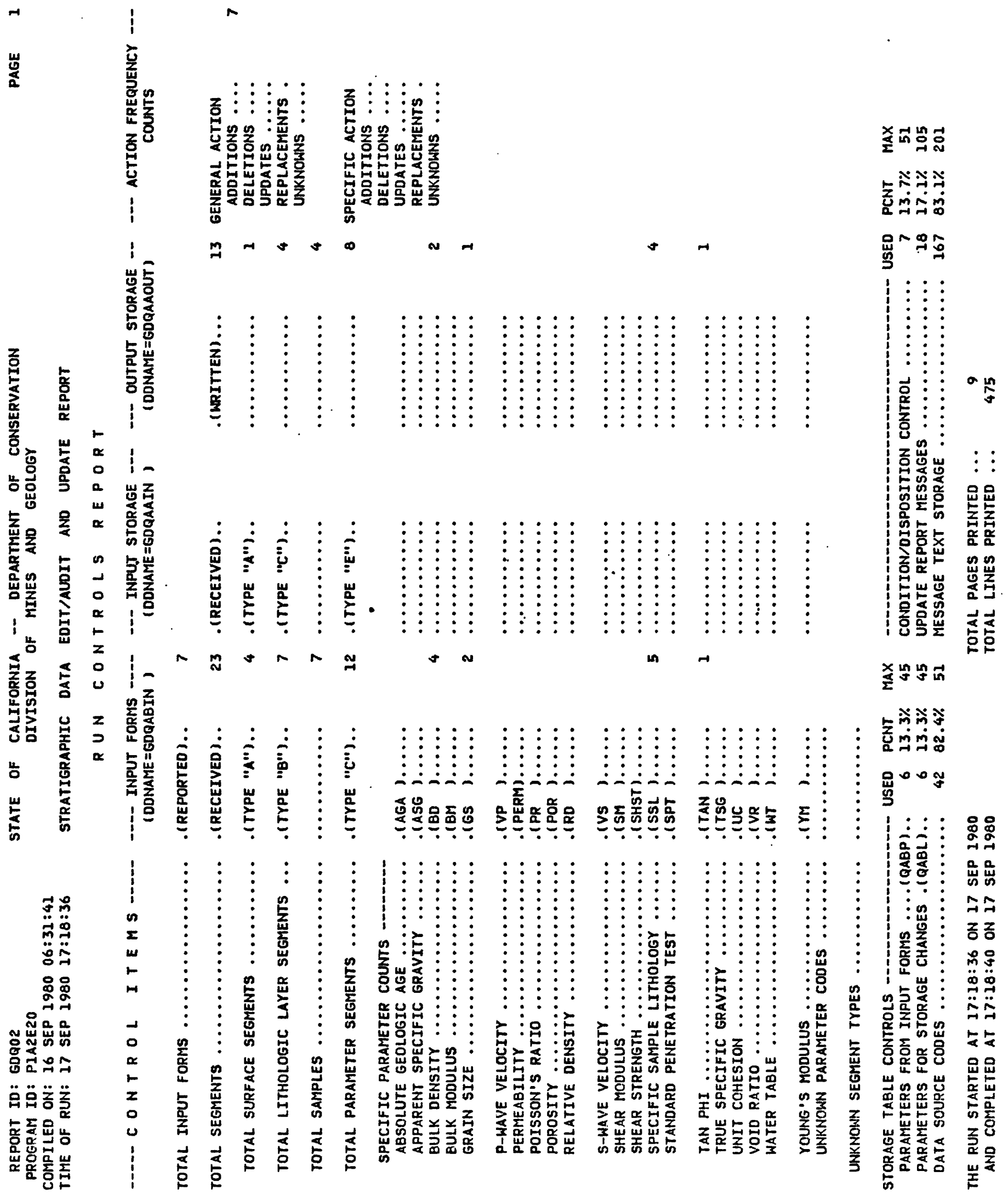




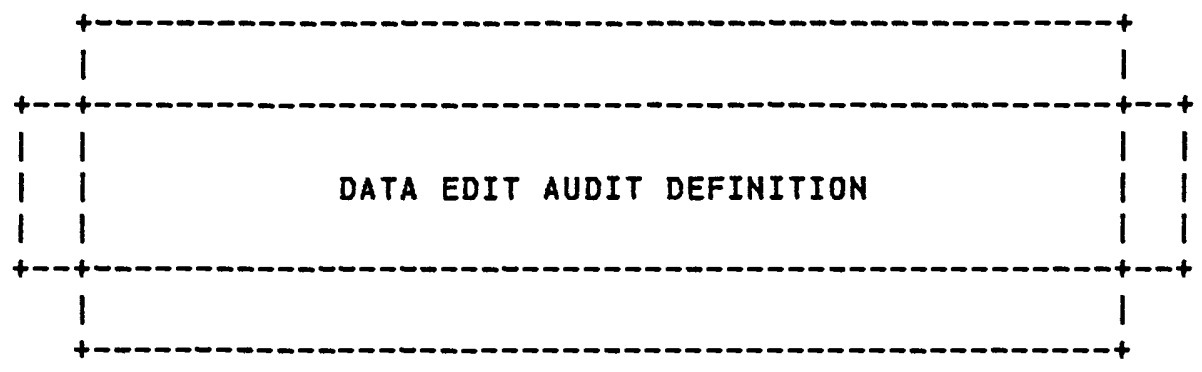


01 STRAT-EDIT-AUDIT-DEF. STRAT-EDIT-AUDIT-DEF

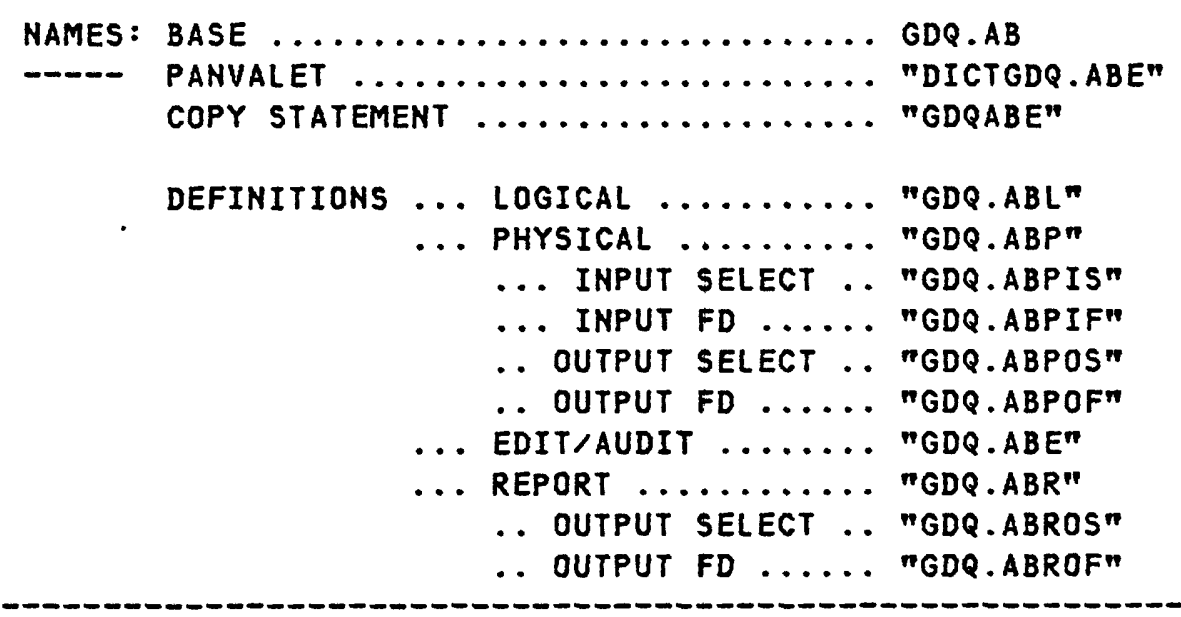

$* 09 / 16 / 80$ *DICTGDQ

* LVOOO

$* 00006$

$* 00007$

$* 00008$

$* 00009$

$* 00010$

$* 00011$

$* 00012$

$* 00013$

$* 00014$

$* 00015$

$* 00016$

$* 00017$

$* 00018$ 
03 QABE-PRIM-SURFACE-DATA-ITEMS $\quad 00020$

05 QABE-CALIF-COORDINATES.

00023

00024

* EDIT

CALIF COORDINATE ZONE =

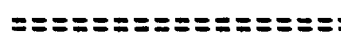

07 QABE-CALIF-COORDNT-ZONE

PIC $x$.

88 QABE-CALIF-COORDNT-ZONE-VLD

VALUE ' I' THRU ' 7 '.

$=00025$

00026

00027

00028

00029

00030

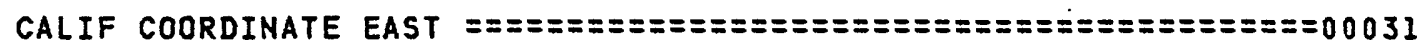

00032

00033

00034

* EDIT

CALIF COORDINATE EAST

$P I C \times(7)$.

00035

88 QABE-CALIF-COORDNT-EAST-VLD

00036

VALUE '0000000' THRU 14400000 '.

00037

* AUdit CALIF COORDINATE EAST

00038

88 QABE-CALIF-COORDNT-ZI-E-VLD

00039

VALUE '0000000' THRU '2567000'.

88 QABE-CALIF-COORDNT-Z2-E-VLD

VALUE ' $0000000^{\prime}$ THRU ' 2705000 '.

88 QABE-CALIF-COORDNT-Z3-E-VLD

VALUE '0000000' THRU '2780000'.

88 QABE-CALIF-COORDNT-Z4-E-VLD

VALUE '0000000' THRU ' 3015000 '.

00040

00041

00042

00043

00044

00045

00046

88 QABE-CALIF-COORDNT-Z5-E-VLD

00047

VALUE '0000000' THRU ' 3170000 '.

00048

88 QABE-CALIF-COORDNT-Z6-E-VLD

00049

VALUE '0000000' THRU '2570000'.

00050

88 QABE-CALIF-COORDNT-Z7-E-VLD

00051

VALUE ' 3980000 ' THRU '4400000'.

00052

00053

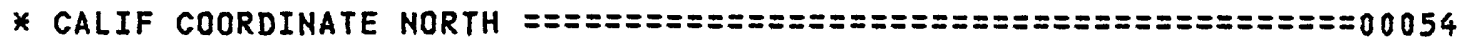

07 QABE-CALIF-COORDNT-NORTH PIC X(7). $\quad 00056$

* EDIT CALIF COORDINATE NORTH

88 QABE-CALIF-COORDNT-NORTH-VLD 00059

VALUE '0000000' THRU '4435000'. 00060

* AUDIT CALIF COORDINATE NORTH

88 QABE-CALIF-COORDNT-ZI-N-VLD 00062

VALUE '0000000' THRU ' 1094000 '. 00063

88 QABE-CALIF-COORDNT-Z2-N-VLD

VALUE '0000000' THRU '1.033000'.

00064

00065

88 QABE-CALIF-COORDNT-Z3-N-VLD

VALUE '0000000' THRU $10911000^{\prime}$.

00066

00067

88 QABE-CALIF-COORDNT-Z4-N-VLD

00068

VALUE '0000000' THRU $10972000^{\prime}$ '.

00069

88 QABE-CALIF-COORDNT-Z5-N-VLD

00070

VALUE '0000000' THRU '1033000'.

00071

88 QABE-CALIF-COORDNT-Z6-N-VLD

00072

VALUE '0000000' THRU '0851000'.

QABE-CALIF-COORDNT-Z7-N-VLD
VALUE ' $3975000^{\prime}$ THRU $14435000^{\prime}$. 
88 QABE-GENERAL-ACTION-NOT-ENTRD

88 QABE-GENERAL-ACTION-CODE-VLD

$$
\text { value ' }
$$

03 QABE-OTHER-SURFACE-DATA-ITEMS.

00088

05 QABE-SURFACE-KEY-DATA-ITEMS.

00089

00090

00091

00092

07 QABE-SURFACE-SEGMENT-DATE-IN.

88 QABE-SURF-SGMT-DATE-NOT-ENTRD VALUE . '.

00093

00094

00095

09 QABE-SURFACE-SEGMENT-DAY PIC XX.

O9 QABE-SURFACE-SEGMENT-MO-ABBR PIC XXX.

00096

09 QABE-SURFACE-SEGMENT-CEN-YR.

11 QABE-SURFACE-SEGMENT-CEN PIC $X X$.

11 PABE-SURFACE-SEGMENT-YR PIC $X X$.

07 QABE-SURFACE-SEGMENT-DATE P.IC $X(8)$.

00097

00098

00099

00100

00101

00102

00103

05 QABE-SURFACE-DETAIL-DATA-ITEMS.

00104

00105

07 QABE-SURFACE-ELEVATION-DATA.

00106

09 QABE-SURFACE-ELEVATION-FLD.

00107

88 QABE-SURFACE-ELEV-NOT-ENTRD VAlue ' '

88 PABE-SURF-POINT-TO-BE-DELET.ED VALUe 'DELETE'.

11 LVL

11 QABE-SURFACE-ELEVATION PIC S9(5)V9.

00108

00109

00110

00111

88 QABE-SURFACE-ELEVATION-VLD

00112

00113

VALUE -282 THRU + 14495 .

11 RDF

11 FILLER REDEFINES QABE-SURFACE-ELEVATION.

00114

00115

00116

00117

00118

13 QABE-SURFACE-ELEVATION-XXXXXX $P I C X 6$
$X X X X X 6$
$P I C X$.

00119

00120

09 QABE-SURFACE-ELEVATION-SIGN PIC $X$.

00121

88 QABE-SURFACE-ELEV-IS-UNSIGNED

VALUe' ' ' +'.

00122

00123

07 QABE-SURFACE-ELEVATION-5-A PIC $X$.

00124

88 QABE-SURFACE-ELEV-S-A-BLANK

00125

VALUe' '

00126

88 QABE-SURFACE-ELEV-S-A-VLD

00127 
NOTE ** NOTE $* *$ NOTE $* *$
07 QABE-2NDRY-SURFACE-DATA-ITEMS. 00129

00130

88 QABE-2NDRY-SURF-ID-NOT-ENTRD 00131

VALUE, '. 00132

00133

00134

THE "I" IN THE HUNDREDS POSITION IS ADDED 00135

IF NO EDIT ERRORS ARE FOUND IN THE INPUT VALUE. 00136

00137

00138

00139

00140

00141

00142

00143

00144

00145

00146

00147

00148

00149

00150

00151

00152

00153

00154

00155

00156

00157

00158

00159

00160

00161

00162

00163

00164

00165

00166

00167

00168

00169

00170

00171

00172

O9 QABE-COUNTY-CODE PIC XX. 00173

88 QABE-COUNTY-CODE-NOT-ENTRD $\quad 00174$

VALUE ' '. 00175

88 PABE-COUNTY-CODE-VLD 00176

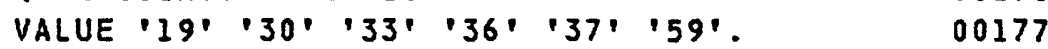

09 QABE-COUNTY-CODE-5-A PIC $X$. 00178

88 PABE-COUNTY-CODE-S-A-BLANK 00179

VALUE ' '. 00180

88 QABE-COUNTY-CODE-S-A-VLD 00181

VAlUe ' $A$ ' ' $D$ ' ' $U$ ' 'R'. 
09 QABE-ATLAS-SHEET-CODE PIC XX.

00186

88 QABE-ATLAS-SHEET-CODE-NOT-ENTR VALUE' '.

88 QABE-ATLAS-SHEET-CODE-VLD

00188

VALUE '07' '08' '19'.

09 PABE-ATLAS-SHEET-CODE-S-A PIC $X$.

88 QABE-ATLAS-SHEET-CODE-S-A-BLNK VALUE ' '.

88 QABE-ATLAS-SHEET-CODE-S-A-VLD

VALUE 'A' ' $D$ ' ' $U$ ' 'R'.

07 QABE-AUXILIARY-INFO-DATA-ITEMS.

00199
00200
00201
00202
00203
00204
00205
00206
00207
00208
00209
00210
00211
00212
00213
00214
00215
00216
00217
00218

07 PABE-PRIM-RELEASE-AUTHORITY

PIC $x$.

00218 
05 QABE-LAYER-KEY-DATA-ITEMS.

07 QABE-DEPTH-TO-TOP-FLD.

88 QABE-DEPTH-TO-TOP-NOT-ENTRD

09 QABE-DEPTH-TO-TOP

88 QABE-DEPTH-TO-TOP-VLD

VALUE O THRU 25000.

07 QABE-DEPTH-TO-TOP-S-A

00226

00227

PIC $9(5)$.

00228

00229

00230

PIC $x$.

00231

00232

00233

00234

00235

00236

00237

07 QABE-LAYER-SEGMENT-DATE-IN.

00238

88 QABE-LAYER-SGMT-DATE-NOT-ENTRD

VALUE '

'.

00239

09 QABE-LAYER-SEGMENT-DAY

PIC XX.

00240

09 QABE-LAYER-SEGMENT-MO-ABBR

PIC XXX.

00241

09 QABE-LAYER-SEGMENT-CEN-YR.

11 QABE-LAYER-SEGMENT-CEN

PIC $X X$.

00242

11 QABE-LAYER-SEGMENT-YR

PIC $X X$.

00244

00245

07 QABE-LAYER-SEGMENT-DATE

PIC $\times(8)$.

00246 
07 QABE-THICKNESS-FLD-AND-S-A.

88 QABE-A-LAYER-IS-TO-BE-DELETED

00252

VALUE 'DELETE'.

09 QABE-THICKNESS-FLD.

00253

00254

88 QABE-THICKNESS-NOT-ENTRD

00255

VALUE' '

00256

11 QABE-THICKNESS

PIC $9(5)$.

00257

88 QABE-THICXNESS-VLD

00258

VALUE 1 THRU 10000.

09 QABE-THICKNESS-S-A

PIC $X$.

00259

00260

88 QABE-THICKNESS-S-A-BLANK

VALUE ' '

00261

00262

00263

88 QABE-THICKNESS-S-A-VLD

VALUE 'A' 'D' 'U' 'R' 'E'.

07 QABE-GENERAL-LITHOLOGY

PIC $X X$.

00264

00265

00266

88 QABE-GENERAL-LITH-NOT-ENTRD

00267

VALUE' '.

00268

00269

88 QABE-GENERAL-LITHOLOGY-VLD

00270

VALUe 'AF' 'BD' 'CG' 'CL' 'GR' 'IG' 'LS' 'MT' 00271 'PT' 'SD' 'SG' 'SH' 'SL' 'SO' 'SR' 'SS' 00272 'VO'.

00273

00274

07 QABE-GENERAL-LITHOLOGY-S-A PIC $X$.

00275

88 QABE-GENERAL-LITH-S-A-BLANK

VALUE' '.

88 QABE-GENERAL-LITHOLOGY-S-A-VLD

VALUE 'A' ' $D$ ' 'U' 'R'.

00276

0.0277

00278

00279

00280

07 QABE-STRAT-ID.

00281

88 QABE-STRAT-ID-NOT-ENTRD

00282

VALUE'

00283

09 QABE-STRAT-GROUP

PIC $X X$.

00284

88 QABE-STRAT-GROUP-NOT-ENTRD

0.0285

88 QABE-STRAT-GROUP-VLD

00286

VALUE' '.

00287

09 QABE-STRAT-FORMATION

PIC XXX.

00288

88 QABE-STRAT-FORMATION-NOT-ENTRD VALUE' '.

00289

00290

00291

00292

00293

09 QABE-STRAT-MEMBER

PIC XXX.

00294

00295

00296

00297

07 QABE-STRAT-ID-S-A

PIC $x$.

00298

00299

00300

VALUE '

00301

88 QABE-STRAT-ID-S-A-VLD

00302 
07 QABE-DETERMINATION-METHOD PIC $X$.
88 QABE-DETRMTN-METHOD-NOT-ENTRD

00306

00307 VALUE' '.

00308

88 QABE-DETERMINATION-METHOD-VLD

00309

VALUE ' 1 ' THRU ' 5 '.

00310

07 QABE-DETERMINATION-METHOD-S-A PIC $X$.

00311

88 QABE-DETRMTN-METHOD-S-A-BLANK

VALUE ' '.

00312

00313

88 QABE-DETRMTN-METHOD-S-A-VLD

00314

VAlUe 'A' ' $D$ ' 'U' 'R'.

00315

00316

07 QABE-REL-GEOL-AGE.

00317

88 QABE-REL-GEOL-AGE-NOT-ENTRD

00318

VALUE ' '.

00319

09 QABE-REL-GEOL-ERA

PIC $x$.

00320

88 QABE-REL-GEOL-ERA-NOT-ENTRD

00321

VALUE ' '

88 QABE-REL-GEOL-ERA-VLD

00322

00323

VALUE ' 1 ' THRU ' 4'.

00324

09 QABE-REL-GEOL-PERIOD

PIC XX.

00325

88 QABE-REL-GEOL-PERIOD-NOT-ENTRD VALUE' '.

00326

00327

88 QABE-REL-GEOL-PERIOD-VLD

00328

VALUE ' 01 ' THRU ' 12 '.

00329

09 QABE-REL-GEOL-SER-EPH

PIC XX.

00330

88 QABE-REL-GEOL-SER-EPH-NOT-ENTR

00331

VALUE' '.

00332

88 QABE-REL-GEOL-SER-EPH-VLD

VALUE 'OI' THRU '07'.

00333

00334

09 QABE-REL-GEOL-STAGE

PIC XXX.

00335

88 QABE-REL-GEOL-STAGE-NOT-ENTRD

00336 VALUE' '.

00337

88 QABE-REL-GEOL-STAGE-VLD

00338

VALUE '001' THRU '008'.

07 QABE-REL-GEOL-AGE-5-A

PIC $x$.

00339

00340

88 QABE-REL-GEOL-AGE-5-A-BLANK

00341

VALUe' '.

00342

88 QABE-REL-GEOL-AGE-S-A-VLD

00343

VALUE 'A' ' $D$ ' ' $U$ ' 'R'.

00344

*--- EN D O F --- LAYER-DATA-ITEMS

00346 
03 QABE-SAMPLE-DATA-ITEMS.

0.0348

*

--- - - - - - - - - - -

00349

00350

05 QABE-SAMPLE-KEY-DATA-ITEMS.

00351

00352

00353

07 QABE-SAMPLE-DEPTH-FLD.

00354

88 QABE-SAMPLE-DEPTH-NOT-ENTRD

00355

VALUE ' '.

O9 QABE-SAMPLE-DEPTH

88 QABE-SAMPLE-DEPTH-VLD

PIC 9(5)V9.

00356

00357

VALUE O THRU 25000.

00358

00359

07 FILLER REDEFINES QABE-SAMPLE-DEPTH-FLD.

00360

09 QABE-SAMPLE-DEPTH-12345X

PIC $X(5)$.

00361

09 QABE-SAMPLE-DEPTH-XXXXX6

PIC $x$.

07 QABE-SAMPLE-DEPTH-S-A

PIC $x$.

00362

00363

00364

VALUe' '.

00365

88 QABE-SAMPLE-DEPTH-S-A-VLD

00366

VALUE 'A' 'D' 'U' 'R'. 
11 LVL

11 RDF

11 RDF

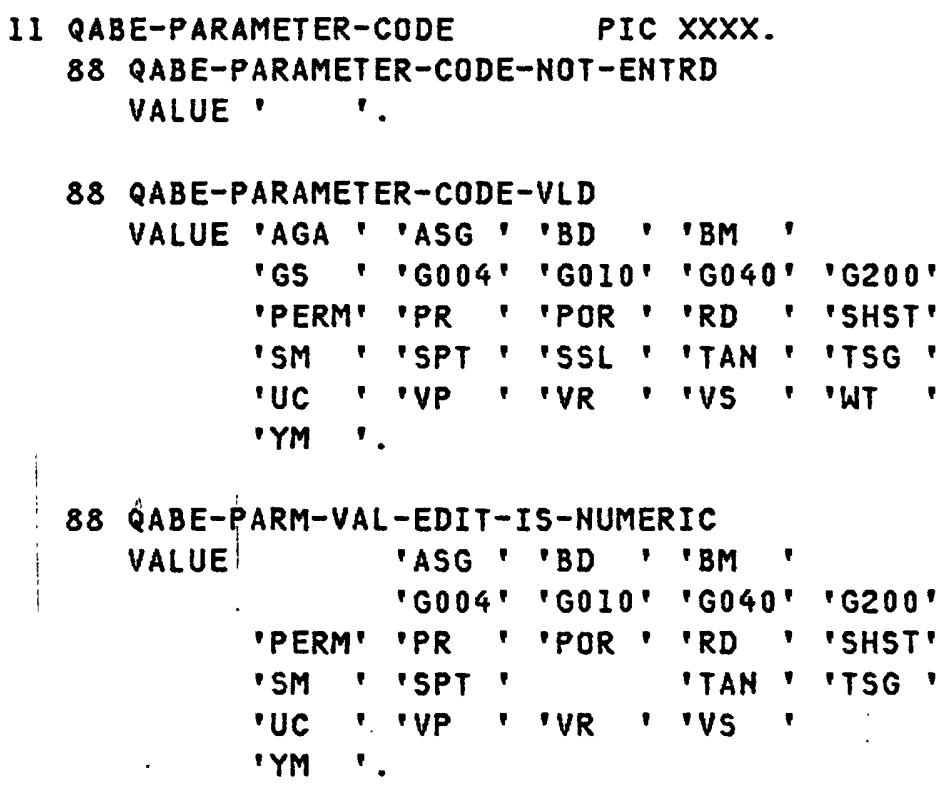

00375

00376

00377

00378

00379

00380

00381

00382

00383

00384

00385

00386

00387

00388

00389

00390

00391

00392

00393

00394

00395

00396

00397

00398

00399

00400

00401

00402

00403

00404

00405

00406

00407

00408

00409

00410

00411

00412

00413

00414

00415

00416

00417

00418

00419

00420

00421

00422

00423 
11 QABE-2NDRY-DATA-SOURCE.

88 QABE-2NDRY-DATA-SORC-NOT-ENTRD

13 QABE-2NDRY-DATA-SOURCE-ID. 00435 15 QABE-2NDRY-SOURCE-CODE

15 QABE-2NDRY-SOURCE-QUALFR PIC XXX. 00437 00438

13 QABE-2NDRY-ACTIVITY-CODE PIC XXX.

00439 PIC $X$. 00440 00441

11 QABE-2NDRY-DATA-SOURCE-S-A 00442 PIC $X$. 00443

88 QABE-2NDRY-DATA-SORC-S-A-BLANK 00444 VALUE' '

00445

88 QABE-2NDRY-DATA-SOURCE-S-A-VLD 00446 VALUE 'A' ' $D$ ' ' $U$ ' 'R'. 00447

00448

* A L L MEASUREMENT DATE IN EDITS -

11 QABE-MEASUREMENT-DATE-IN. 00450

88 QABE-MEASURMT-DATE-NOT-ENTRD. VALUE' ' 00451 0.0452

13 QABE-MEASUREMENT-DAY PIC $X X$.

13 QABE-MEASUREMENT-MO-ABBR 00453 00454 0045.5

00456

13 QABE-MEASUREMENT-CEN-YR. PIC XXX.

00457 15 QABE-MEASUREMENT-CEN 00458 PIC XX. $\quad 00459$ 15 QABE-MEASUREMENT-YR PIC XX. 00460

11 QABE-MEASUREMENT-DATE-S-A PIC $X$. 00461 88 QABE-MEASURMT-DATE-S-A-BLANK 00462 VALUe' '. 00463

88 QABE-MEASUREMENT-DATE-S-A-VLD 00464 VALUE 'A' ' $D$ ' ' $U$ ' 'R'. 00465

11 QABE-MEASUREMENT-DATE PIC $\times(8) .00467$ 00468 * A L L DMG INTERPRETED CODE EDITS

11 QABE-DMG-INTRPTD-CODE PIC $X$. 00470 00471 88 QABE-DMG-INTRPTD-CODE-NOT-ENTR 00472 00473 VALUE' '

88 QABE-DMG-INTRPTD-CODE-VLD

11 QABE-DMG-INTRPTD-CODE-S-A PIC $X$.

88 QABE-DMG-INTRPTD-CODE-S-A-BLNK VALUE' '

88 QABE-DMG-INTRPTD-CODE-S-A-VLD

VALUe 'A' 'D' ' $U$ ' 'R'. 
* A L L RELEASE AUTHORITY EDITS -

11 QABE-RELEASE-AUTHORITY PIC X.
88 QABE-RELEASE-AUTH-NOT-ENTRD 00485 00486 VALUE ' ' 00487 00488

88 QABE-RELEASE-AUTHORITY-VLD VALUE ' I' THRU ' 4 '. 00489 00490

11 QABE-RELEASE-AUTHORITY-S-A 00491 00492

88 QABE-RELEASE-AUTH-S-A-BLANK 00493 VALUE ' '. 00494

88 QABE-RELEASE-AUTHORITY-S-A-VLD 00495 VALUe 'A' 'D' 'U' 'R'. 00496 
II LVL

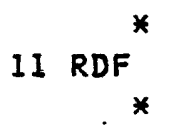

*

* aga value edit -- See "multiple-numeric-edit-DeFn beloW -

* ASG

* BD

* BM

* GS

* PERM

* por value edit

* PR

* SHST

* SM

* SPT

VALUE EDIT

1 QABE-SINGLE-NUMERIC-EDIT-DEF

ABE SINGLE-NUMERIC-ED

REDEFINES QABE-PARM-VALUE-EDIT-DEF

PIC $9(8) \vee 9(7)$.

00500

00501

00502

00503

00504

00505

00506

00507

00508

00509

$-00510$

00511

VALUE EDIT

88 QABE-ASG--VALUE-VLD

VALUE 1 THRU 3.

00512

00513

00514

VALUE EDIT

88 QABE-BD---VALUE-VLD

VALUE 25 THRU 190.

00516

00517

VALUE EDIT

88 QABE-BM---VALUE-VLD

00518

00519

00520

VALUE 0 THRU 2000.

00521

00522

00523

VALUE 1 THRU 100.

$-00524$

00525

00526

VALUE O THRU 25000.

88 QABE-POR--V.ALUE-VLD

VALUE O THRU 99.

00528

00529

VALUE EDIT

88 QABE-PR-- VALUE-VLD

VALUE .2 THRU .5.

00531

00532

VALUE EDIT

88 QABE-RD---VALUE-VLD

00534

VALUE 1 THRU 99.

00535

VALUE EDIT

88 QABE-SHST-VALUE-VLD

00537

VALUE O THRU 12000.

00538

VALUE EDIT

88 QABE-SM---VALUE-VLD

VALUE O THRU 1000 .

00541

$-00542$

00543

00544

00545

VALUE 1 THRU 70.

$-00546$

00547

* TAN

VALUE EDIT -- SEE "NON-NUMERIC-EDIT-DEF" BELOW

00548

88 QABE-TAN--VALUE-VLD

00549

VALUE O THRU 1.3.

00550

* TSG
88 QABE-TSG--VALUE-VLD

VALUE O THRU 10. 


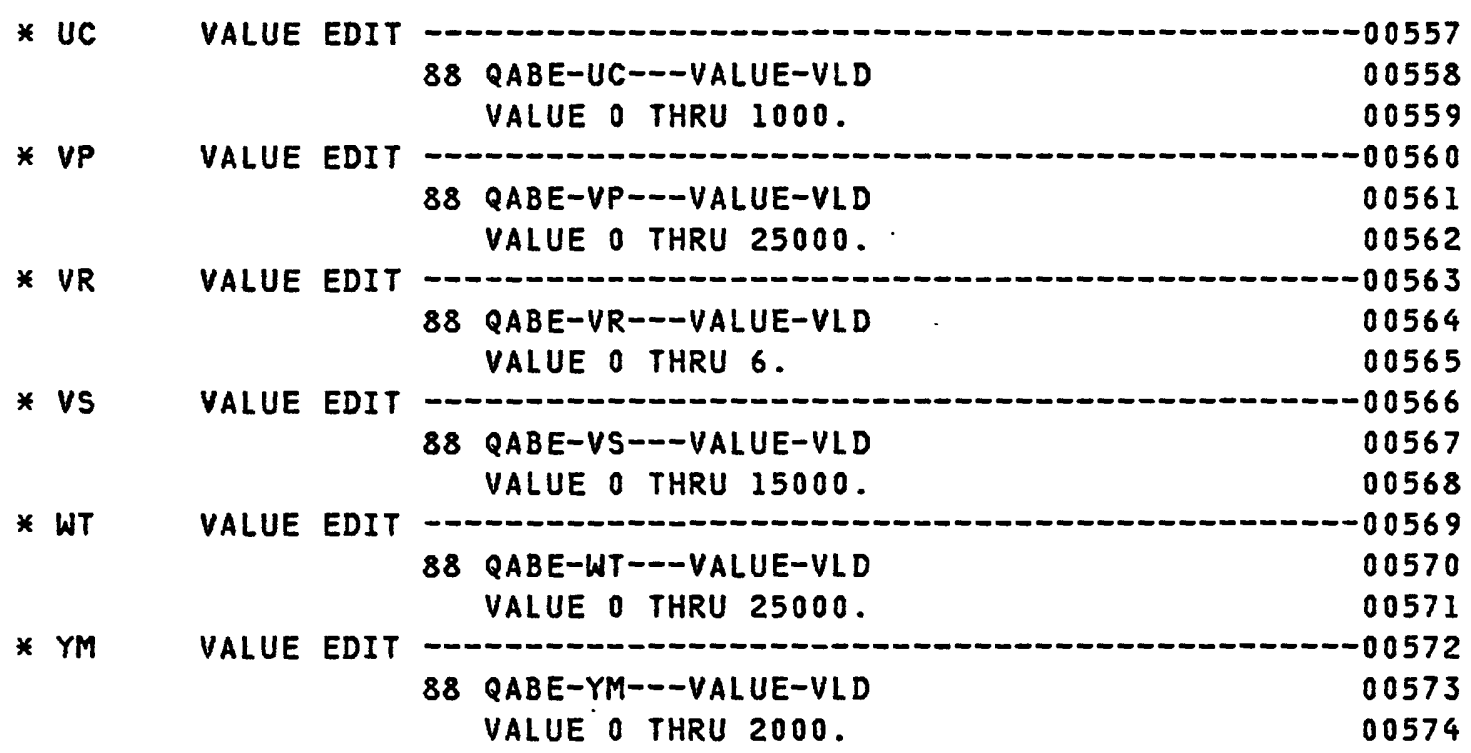

II RDF

11 FILLER

REDEFINES QABE-PARM-VALUE-EDIT-DEF.

00576

00577

00578

*

$13 \mathrm{LVL}$

13 QABE-MULTPLE-NUMERIC-EDIT-DEF

00579

*

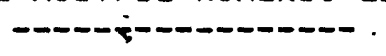

00580

00581

PIC $\times(15)$.

00582

00583

* AGA

VALUE EDIT

13 RDF

13 QABE-AGA--VALUE-EDIT-DEF

REDEFINES QABE-MULTPLE-NUMERIC-EDIT-DEF.

15 QABE-AGA--BASE-EDIT-DEF

PIC 9V99.

88 QABE-AGA--BASE-VALUE-VLD

VALUE O THRU 9.99.

15 QABË-AGA--UNCERT-EDIT-DEF

PIC 9V99.

88 QABE-AGA--UNCERT-VALUE-VLD

$-00584$

00585

00586

00587

00588

00589

00590

00591

00592

VALUE O THRU 9.99.

00593

00594

15 QABE-AGA-DXPONENT-PREFIX 00595

PIC $X$. $\quad 00596$

88 QABE-AGA--EXPONENT-PREFIX-VLD 00597

VALUE 'E'.

00598

15 QABE-AGA--EXPONENT-EDIT-DEF 00599

PIC 9. $\quad 00600$

88 QABE-AGA--EXPONENT-VALUE-VLD 00601

VALUE 2 THRU $9 . \quad 00602$ 
11 RDF

$13 \mathrm{LVL}$

* * SSL

13 RDF
11 FILLER

REDEFINES QABE-PARM-VALUE-EDIT-DEF.

00606

00607

00608

00609

00610

00611

00612

00613

PIC $X(8)$.

00614

13 QABE-SSL--VALUE-EDIT-DEF

REDEFINES QABE-NON-NUMERIC-EDIT-DEF

00615

00616

PIC $X(8)$.

00617

88 QABE-SSL--VALUE-VLD VALUE 00618

*

C L A Y S

- $C$

- CMS

- CD

- CO

'CY

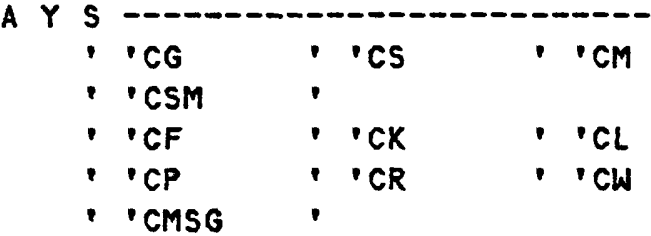

00619

.00621

00622

.00623

.00624

00625

00626

*

- $R$ A V E L S

' $G$

' GS

$-00627$

- GSM

' GB

$+$

- -GF

' GW

- $G X$

- GS

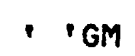

' GC

.00628

00629

.00630

G

- 'Gl

- 'gR

.00631

.00632

00633

*

- S A N D S

's

- SMC

' $5 B$

'so

- GM

- GC

00634

.00635

- ISG

- I $5 \mathrm{C}$

- 'scG

.00636

- 'SCM

- ISL

.00637

'sx

- ISP

- isY

- 'sW

.00638

00639

00640

*

-- S I L T S

$$
\text { 'M }
$$

MCS

'MF

'MP

'MY

: MG
'MSC
'MK
'MR

- "ML

, MC

$-00641$

00643

.00644

.00645

00646

00647

*

M I S C
'A
'X
'R
'Y
'BX
'OM
'PM

$-00648$

.00650

.00651

00652

.00653

.00654

00655
.00649 
11 RDF

11 FILLER

REDEFINES QABE-PARM-VALUE-EDIT-DEF.

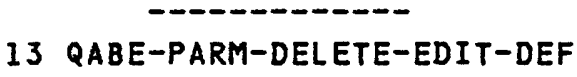

13 QABE-PARM-DELETE-EDIT-DEF

PIC $x(8)$
00659

00660

00661

00662

00663

00664

00665

00666

* delete value edit

88 QABE-A-PARM-IS-TO-BE-DELETED

VALUE 'DELETE '.

\section{7}

00668

00669

* "S An value edit

11 QABE-PARM-VALUE-S-A

88 QABE-PARM-VALUE-S-A-BLANK

VALUE '

88 QABE-PARM-VALUE-S-A-VLD

VALUE 'A' ' $D$ ' 'U' 'R'. 
11 QABE-METHOD

PIC XX.

00680

88 QABE-METHOD-NOT-ENTRD

00681

VALUE' '

00682

00683

* AGA METHOD EDIT

88 QABE-AGA--METHOD-VLD

00684

VALUE 'AA' 'AB' 'AC' 'AD' 'AE' 'AF' 'AG' 00686

'AH' 'AJ' 'AK'. 00687

* ASG METHOD EDIT -

88 QABE-ASG-METHOD-VLD 00689

VALUE 'RO'. 00690

* BD METHOD EDIT -

88 QABE-BD--METHOD-VLD 00692

VALUE 'ST'. $\quad 00693$

* BM METHOD EDIT -

88 QABE-BM---METHOD-VLD 00695

VALUe 'LD' 'IS' 'CA'. 00696

* GS METHOD EDIT -

88 QABE-GS-D-METHOD-VLD 00698

VALUE 'ST'. 00699

* PERM METHOD EDIT -

88 QABE-PERM-METHOD-VLD 00701

VALUE. 'LD' 'OE' 'PT' 'WP'. 00702

* POR METHOD EDIT -

88 QABE-POR--METHOD-VLD 00704

VALUE 'RO'. 00705

* PR METHOD EDIT -

88 QABE-PR-D-METHOD-VLD 00707

VALUE 'LD' 'IS' 'CA'. 00708

* RD METHOD EDIT -

88 QABE-RD---METHOD-VLD 00710

VALIJE 'RO'. 00711

* SHST METHOD EDIT -

88 QABE-SHST-METHOD-VLD 00713

VALUE 'CA' 'IM'. 00714

* SM METHOD EDIT -

88 QABE-SM--METHOD-VLD $\quad 00716$

VALUE 'LD' 'IS' 'CA'. 00717

* SPT METHOD EDIT -

88 QABE-SPT-METHOD-VLD 00719

VALUE 'ST'. $\quad 00720$

* SSL METHOD EDIT -

88 QABE-SSL-METHOD-VLD 00722

VALUE 'DO'. 00723

* TAN METHOD EDIT -

88 QABE-TAN--METHOD-VLD $\quad 00725$

VALUE 'CA'. 00726

* TGS METHOD EDIT -

88 QABE-TSG-METHOD-VLD 00728

VALUE 'RO'. 00729

* UC METHOD EDIT -

88 QABE-UC-D-METHOD-VLD 00731

VALUE 'IM'. 
88 QABE-VP---METHOD-VLD

00736

VALUe 'LD' 'VA' 'VB' 'VC' 'VD' 'VE' 'VF'

'VG' 'VH'.

00738

00739

88 QABE-VP---SPECIAL-METHODS 00740

VALUE 'VA' 'VF' 'VG' 'VH'. 00741

* VR METHOD EDIT

88 QABE-VR-D-METHOD-VLD 00743

VALUE 'RO'. 00744

* VS METHOD EDIT -

88 QABE-VS---METHOD-VLD 00746

VALUE 'LD' 'VA' 'VB' 'VC' 'VD' 'VE' 'VF' 00747 'VG' 'VH'. 00748

88 QABE-VS---SPECIAL-METHODS 00749

VALUE 'VA' 'VF' 'VG' 'VH'. 00750

* WT METHOD EDIT -

88 QABE-WT---METHOD-VLD 00752

VALUE 'DO' 'RE' 'SR'. 00753

88 QABE-WT---SPECIAL-METHOD 00754

VALUE 'SR'.

* YM METHOD EDIT -

88 QABE-YM---METHOD-VLD 00757

VALUE 'LD' 'IS' 'CA'.

11 QABE-METHOD-S-A

PIC X.: $\quad .00760$

88 QABE-METHOD-S-A-BLANK 00761

VALUE ' '. 00762

88 QABE-METHOD-S-A-VLD 00763

VALUE 'A' ' $D$ ' ' $U$ ' 'R'. 00764

* 


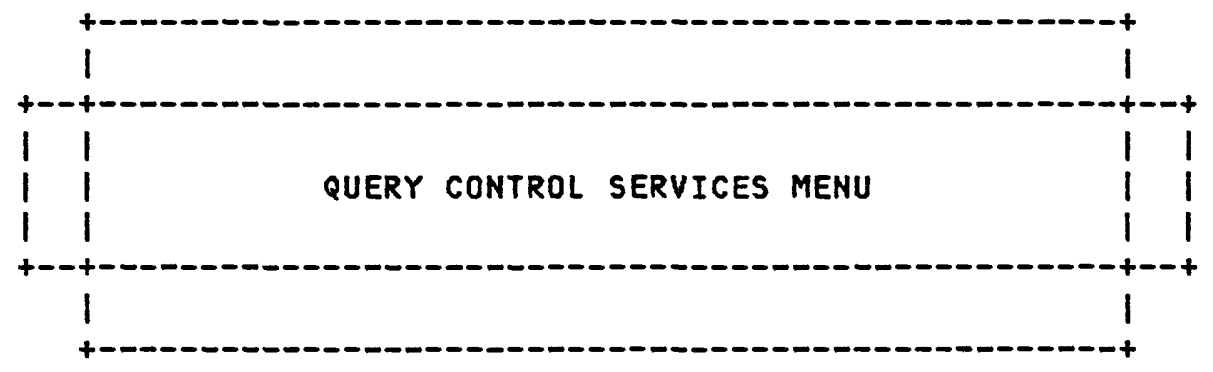


OI QUERY-CONTROL-SERVICES-MENU.

$* * *$

QUERY-CONTROL-SERVICES-MENU - - - CURRENT AS OF 04 APR 1980 (DEF REF: $510 . A 01$ )

03 QUERY-ALL-HISTORY.

PIC XXX.

03 PRINT-RUN-PROFILE-ONLY

PIC XXX.

PIC XXX. 


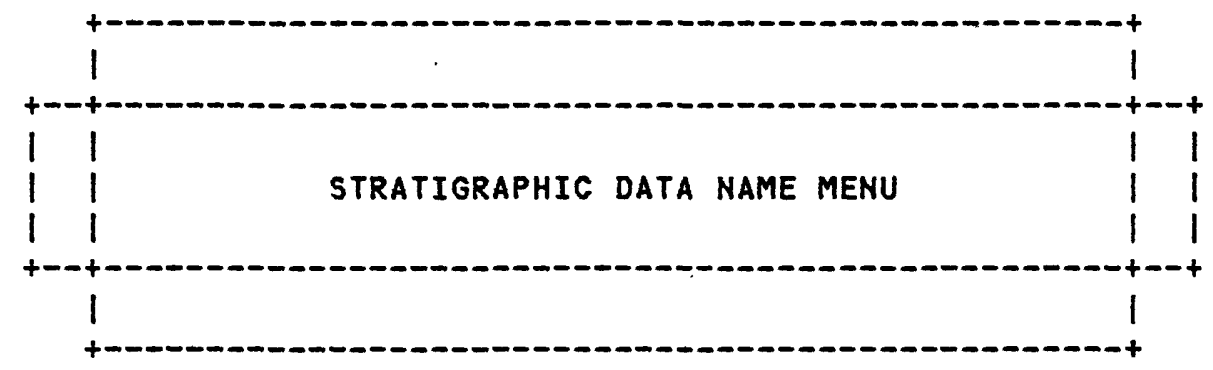




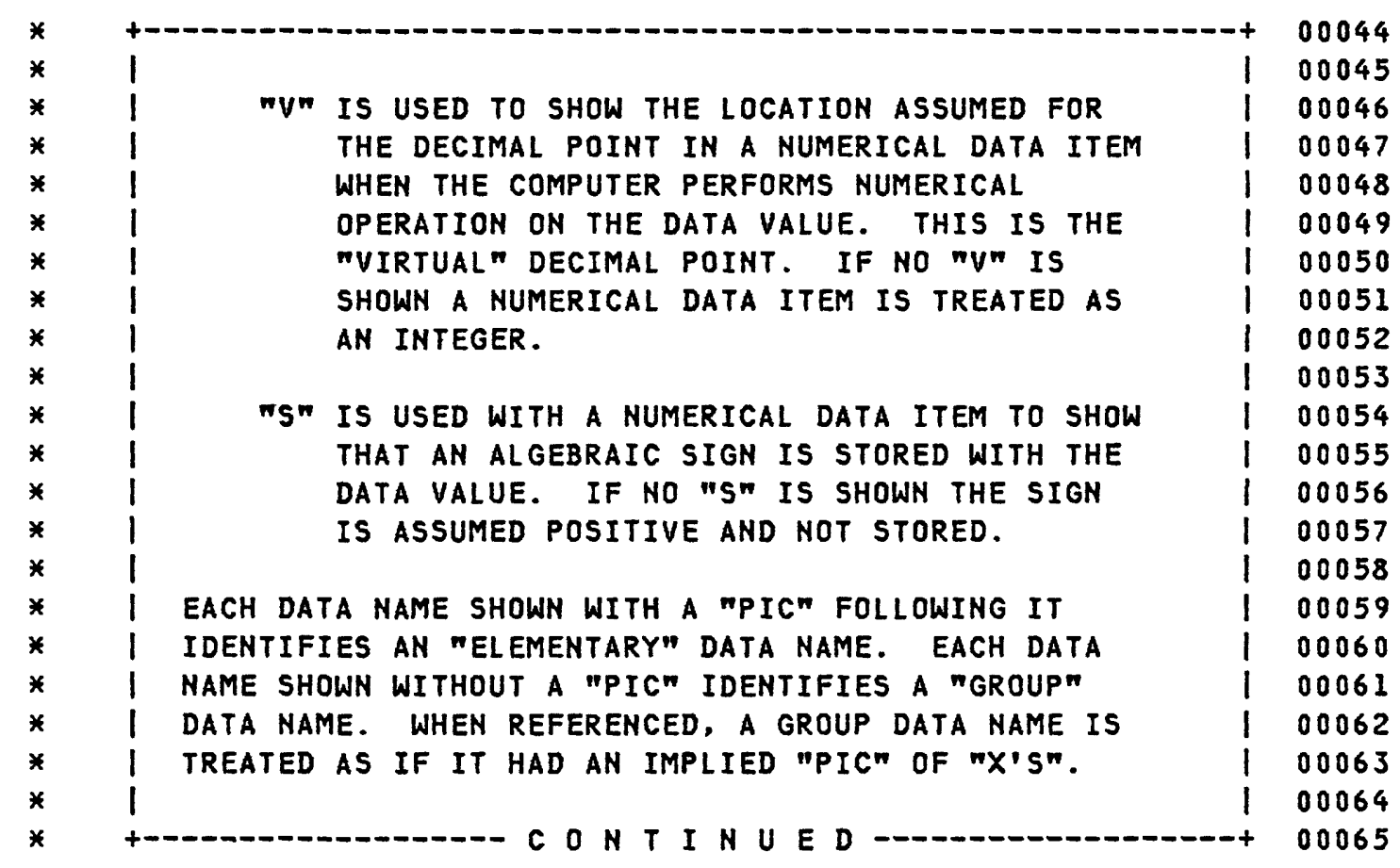


$$
\text { * }
$$

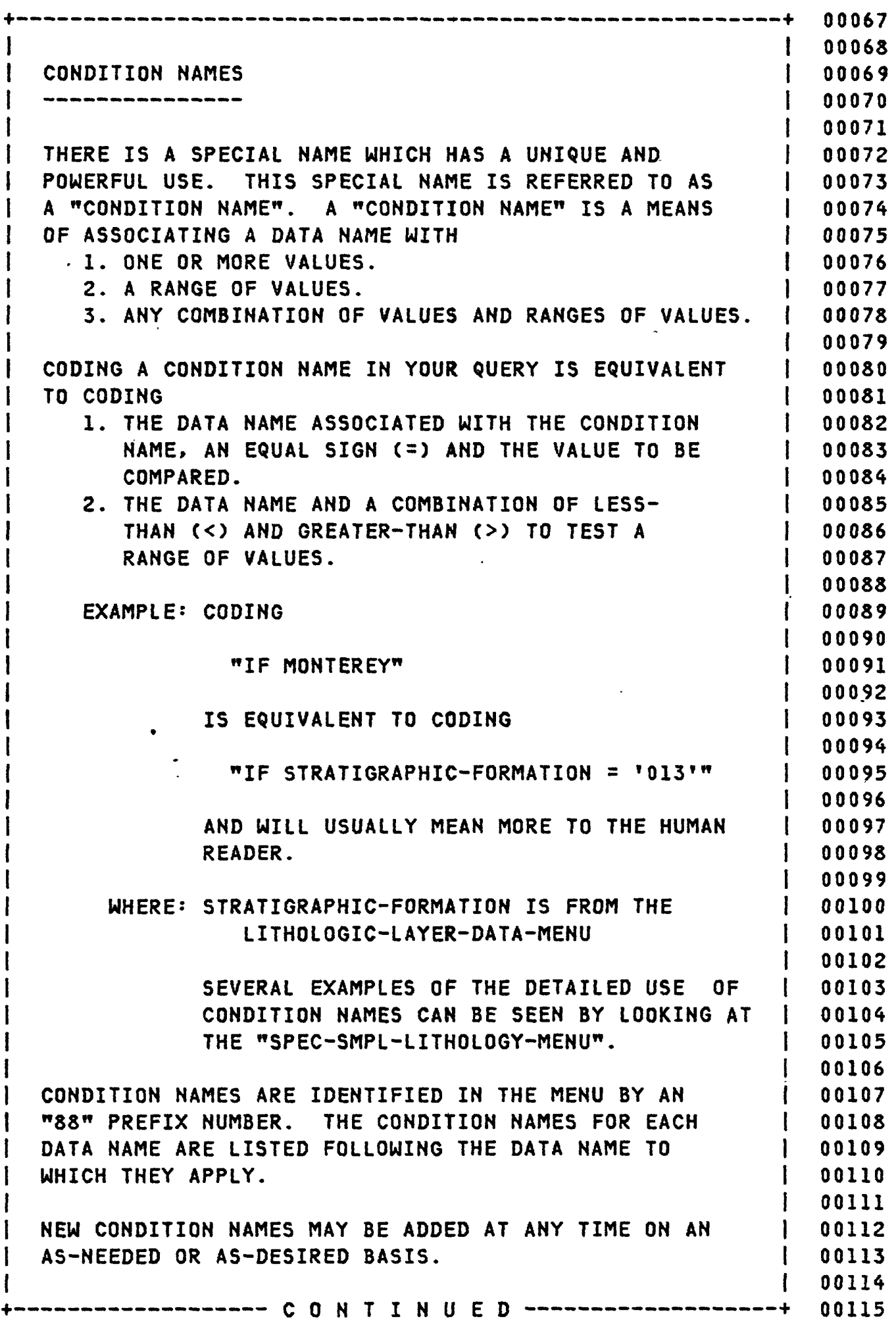




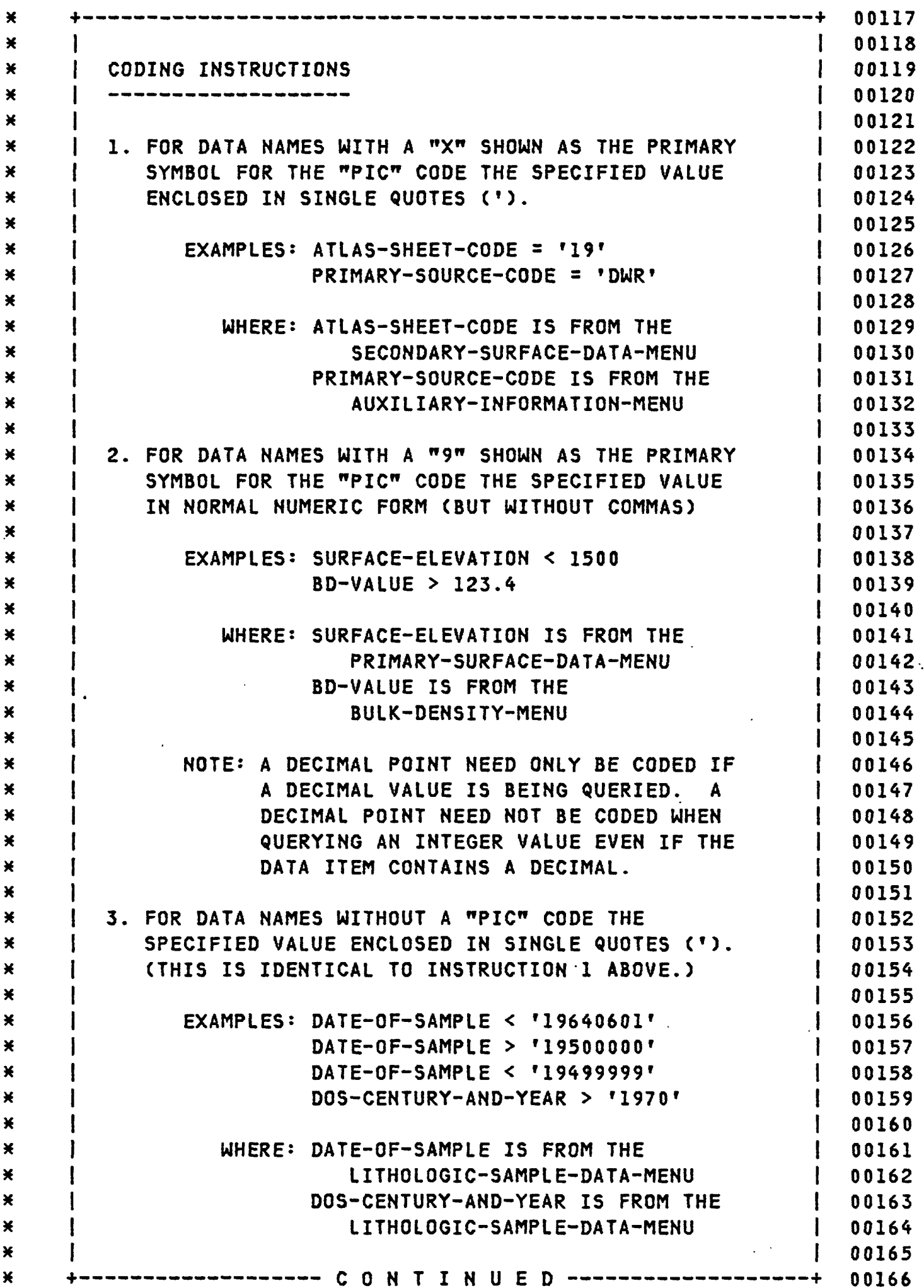


4. PRIOR TO CODING A QUESTION ABOUT A PARTICULAR

00168 NUMERIC DATA ITEM ("PIC" OF "9'5") SUCH AS A GEOTECHNICAL PARAMETER VALUE YOU MUST MAKE SURE THAT THE DATA ITEM VALUE IS IN FACT AVAILABLE.

ASSOCIATED WITH EACH NUMERIC DATA ITEM AND GEOTECHNICAL DATA NAME GROUP IS A CONDITION NAME WHICH SPECIFIES WHETHER OR NOT THE DATA ITEM IS AVAILABLE.

THE CONDITION NAME FOR ANY NUMERIC DATA ITEMS MUST BE CODED PRIOR TO CODING THE NUMERIC DATA NAME IT REFERENCES.

THE CONDITION NAME FOR ANY GEOTECHNICAL PARAMETER MAY ALSO BE CODED WHEN CODING QUERIES FOR A PARTICULAR GEOTECHNICAL PARAMETER DATA NAME GROUP.

EXAMPLE: IF DEPTH-TO-TOP-AVAIL AND DEPTH-TO-TOP $<200$

WHERE: DEPTH-TO-TOP-AVAIL AND DEPTH-TO-TOP ARE FROM THE LITHOLOGIC-SAMPLE-DATA-MENU

EXAMPLE: IF BM-VALUE-AVAIL AND BM-VALUE > 125

WHERE: BM-VALUE-AVAIL AND BM-VALUE ARE FROM THE BULK-MODULUS-MENU

5. THE NEGATION OF A CONDITION, I.E. REQUESTING THAT AN OUTPUT SERVICE BE PROCESSED IF THE CONDITION IS NOT FOUND, MAY ALSO BE CODED. THE RULES FOR CODING FOLLOW CONVENTIONAL SET LOGIC RULES FOR NEGATION.

EXAMPLE: IF NOT CALIFORNIA

IF NOT (CALIFORNIA OR OFFSHORE)

IF NOT (CALIFORNIA OR OFFSHORE)

AND (GL-IS-LIMESTONE OR ANY-REFLECTION-MEASUREMENT) 
*

\section{CODE FORMATTING CONVENTIONS}

THE FORMAT YOU USE TO CODE YOUR QUERY IS GENERALLY

FREE FORMAT. THE RESTRICTIONS ARE MINIMAL.

1. THE FIRST CHARACTER MUST BE BLANK OR AN ASTERISK ( $" * n)$. THE USE OF AN ASTERISK AS THE FIRST CHARACTER DENOTES A COMMENT LINE.

2. CODE YOUR QUERY IN POSITIONS 2 THRU 61 OF EACH LINE ONLY. ALL CHARACTERS FROM POSITION 62 TO THE END (END $=80$ ) ARE IGNORED.

3. DO NOT SPLIT A DATA NAME BETWEEN LINES. IF, DURING THE CODING OF YOUR QUERY, YOU FIND THAT A DATA NAME EXTENDS BEYOND POSITION 61 START THAT DATA NAME ON THE NEXT LINE.

4. IF YOUR QUERY INVOLVES THE MIXING OF "AND'S" AND "OR'S" USE PARENTHESES TO INSURE THAT YOUR QUERY IS PROPERLY INTERPRETED. THE PARENTHESES ARE USED TO GROUP CONDITIONS AND FOLLOW CONVENTIONAL SET LOGIC RULES FOR EVALUATION.

THE USE OF COMMENT AND BLANK LINES

BY USING COMMENT LINES YOUR CAN CREATE YOUR OWN MENUS. BY PLANNING THE STRUCTURE AND CONTENT OF YOUR QUERY YOU CAN UTILIZE THE ASTERISK IN THE FIRST POSITION OF EACH LINE TO "TURN OFF AND ON" ANY LINE. THIS CAN BE USED TO "FINE TUNE" YOUR QUERY BY EITHER INCREASING OR DECREASING THE DATA ITEMS PRINTED. THIS CAN ALSO BE USED TO CONTROL THE DETAIL AND CONTENT OF THE QUERY CONDITIONS SPECIFIED.

COMMENTS MAY BE USED TO START EACH QUERY WITH A MORE ENGLISH DESCRIPTION OF YOUR QUERY THEN PERMITTED BY THE QUERY LANGUAGE CODING REQUIREMENTS AND LIMITATIONS. YOU MAY, FOR EXAMPLE, WANT TO SPECIFY WHY AND FOR WHOM THE QUERY IS BEING REQUESTED, WHERE THE RESULTS SHOULD BE SENT, OR EVEN WHAT YOUR NEXT QUERY MIGHT BE AS A REMINDER DEPENDING ON THE RESULTS OF YOUR CURRENT QUERY. 


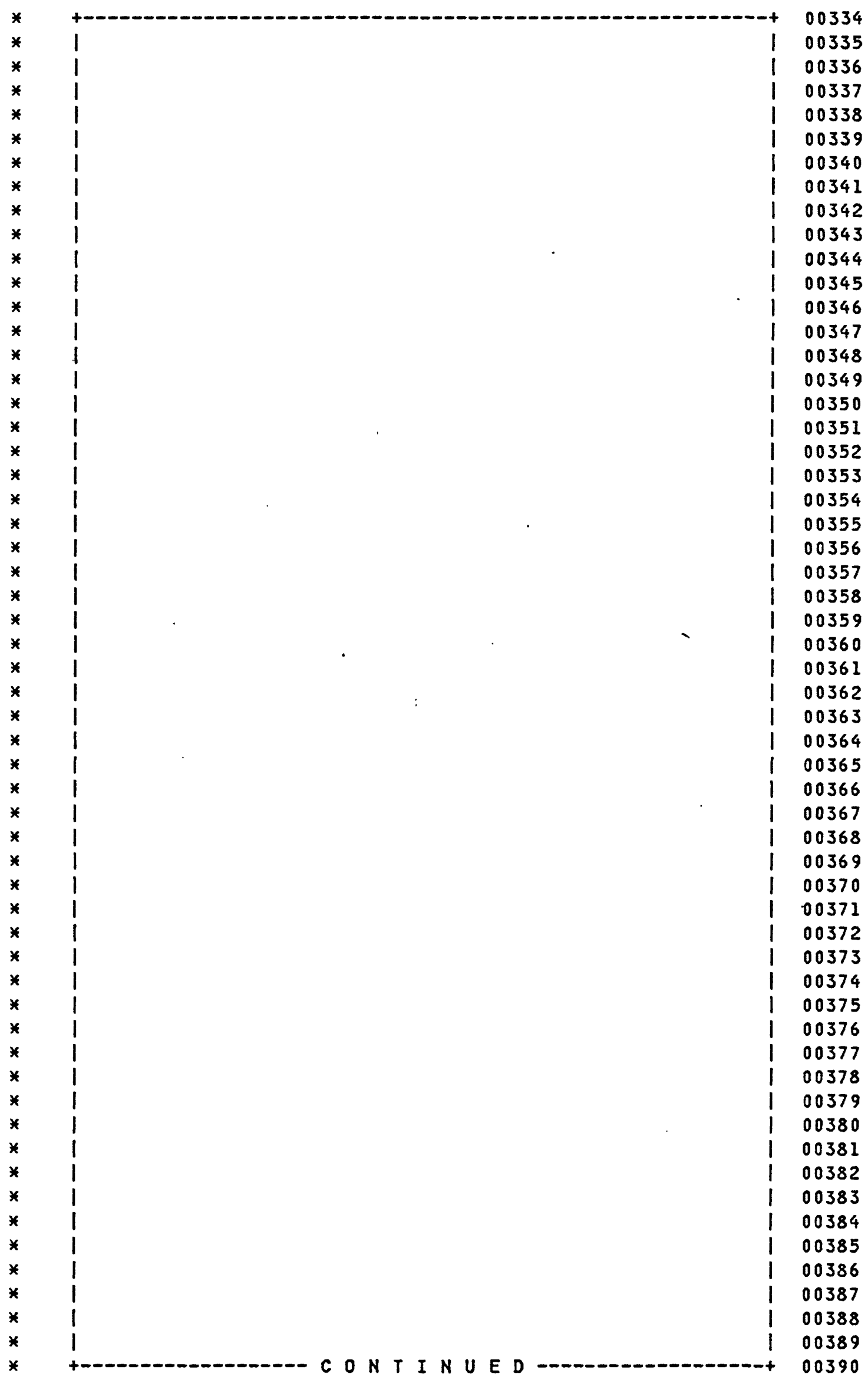


兴

$$
\text { 兴 }
$$

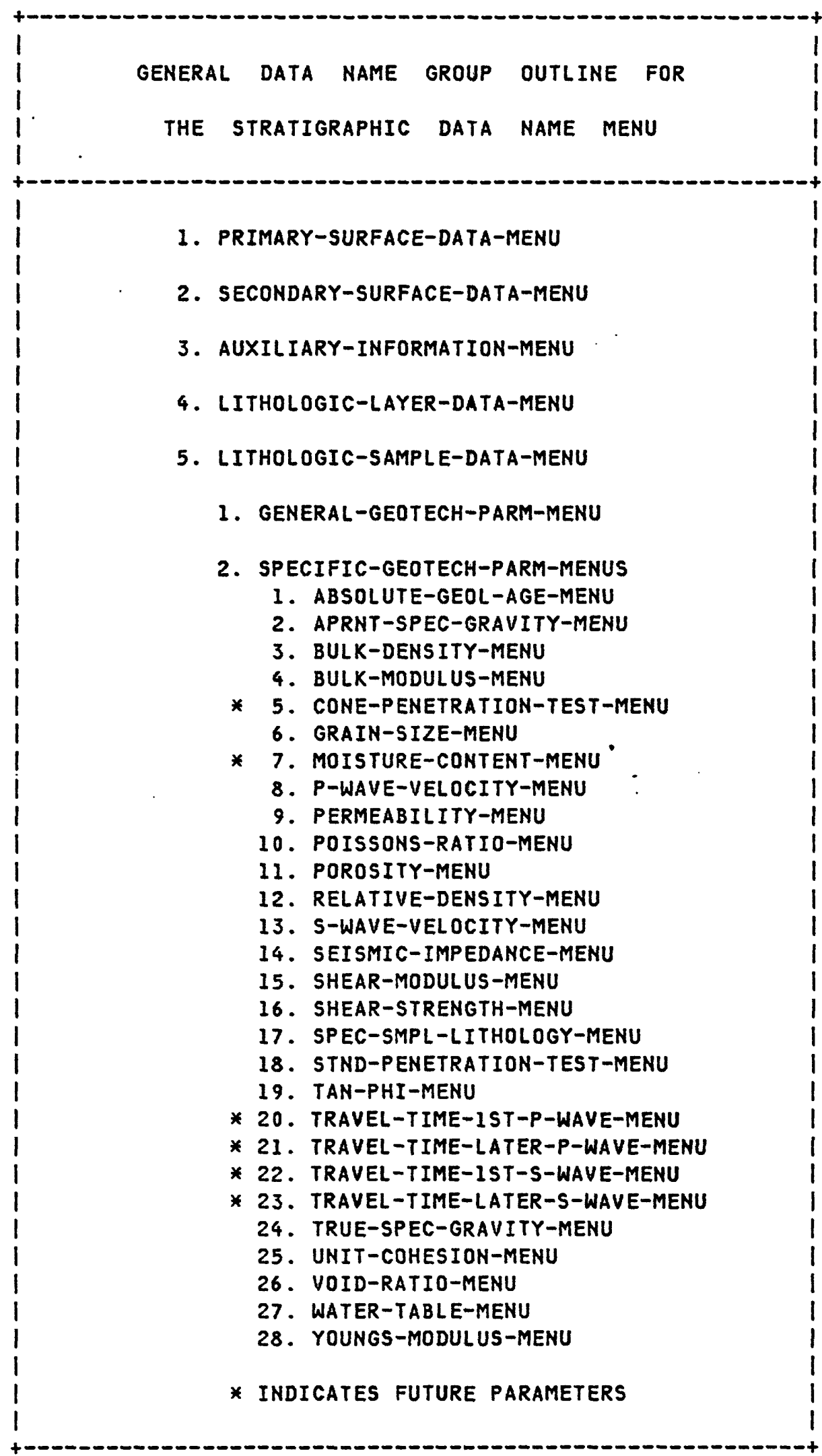

00392

00393

00394

00395

00396

00397

00398

00399

00400

00401

00402

00403

00404

00405

00406

00407

00408

00409

00410

00411

00412

00413

00414

00415

00416

00417

00418

00419

00420

00421

00422

00423

00424

00425

00426

00427

00428

00429

00430

00431

00432

00433

00434

00435

00436

00437

00438

00439

00440

00441

00442

00443

00444 


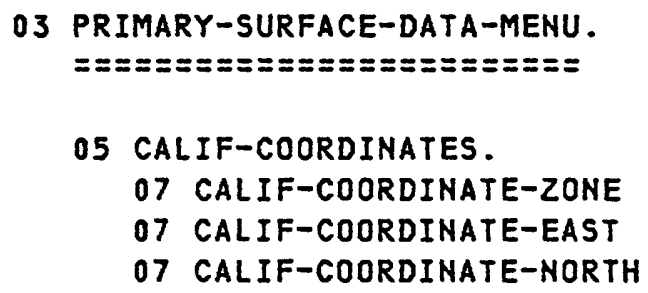

05. IND-SAMPLE-IS-IN-THE-POLYGON
PIC $X$.

PIC $X(7)$.

PIC $X(6)$.
00446

00447

00448

00449

00450

00451

00452

00453

00454

PIC $x$.

00455

00456

00457

* 00458

* 00459

* 00460

* 00461 00462 00463 00464 00465 00466 00467

PIC $x$. VALUE ' $Y$ '. $\quad 00468$ 88 SURFACE-ELEVATION-AVAIL 05 SURFACE-ELEVATION

PIC S9(5)V9.

03 SECONDARY-SURFACE-DATA-MENU.

05 IND-LONGITUDE-AVAIL 88 LONGITUDE-AVAIL

05 LONGITUDE

PIC $X$. 00472 00473 00474 VALUE ' $Y$ '. 00475 PIC 999V9(6). 00476

05 IND-LATITUDE-AVAIL 88 LATITUDE-AVAIL

05 LATITUDE

PIC $x$. 00477 00478 VALUE ' $Y$ '. 00479 PIC 99V9(6). 00480

05 STATE-CODE

88 ARIZONA

88 CALIFORNIA

88 MEXICO

88 NEVADA

88 OREGON

88 OFFSHORE

05 COUNTY-CODE

88 LOS-ANGELES

88 ORANGE

88 RIVERSIDE

88 SAN-BERNARDINO

88 SAN-DIEGO

88 NOT-DETERMINED

PIC XX.

00481

00482

VALUE 'AZ'. 00483

VALUE 'CA'. 00484

VALUE 'MX'. $\triangle 00485$

VALUE 'NV'. 00486

VALUE 'OR'. 00487

VALUE '00'. 00488

PIC XX.

00489

00490

VAlUe ' 19 '. 00491

VALUE ' $30^{\circ}$. 00492

VALUE ' $33^{\prime}$. 00493

VALUE ' $36^{\prime}$. 00494

VALUE ' $37^{\prime}$. 00495

VALUE '59.' 00496

05 ATLAS-SHEET-CODE

88 LONG-BEACH-SHEET

88 LOS-ANGELES-SHEET

88 SANTA-ANA-SHEET

PIC $X X$.

VALUE '07'. 00499

VALUE '08'. 00500

VALUE $19^{\circ}$. 00501 
03 AUXILIARY-INFORMATION-MENU.

00503

O5 PRIMARY-DATA-SOURCE.

07 PRIMARY-DATA-SOURCE-ID.

88 BEACH-LEIGHTON-AND-ASSOC

PIC XXX.

00509

88 BECHTEL-INC

value 'bla'.

00510

88 CALIF-DEPT-OF-TRANSPORTAION

VALUE 'BEC'.

00511

88 CALIF-DEPT-OF-WATER-RESOURCES

VALUE 'CDT'.

00512

88 CALIF-DVSN-OF-MINES-AND-GEOL

88 CALIF-DVSN-OF-OIL-AND-GAS

VALUE 'DWR'.

00513

88 CALIF-OFFICE-OF-ARCH-AND-CNST

VALUE 'DMG'.

00514

88 CALIF-STATE-LANDS-COMMISSION

88 CHEVRON-USA-INC

88 CONVERSE-DAVIS-DIXON

88 ENGINEERING-GEOLOGY-CONSULT

88 FUGRO-INC

88 GETTY-OIL-COMPANY

$88 \mathrm{H}-\mathrm{V}$-LAWMASTER-AND-CO-INC

VALUe 'DOG'.

00515

VALUE 'OAC'.

00516

00517

VALUE 'SLC'.

00518

VALUE 'CHV'.

00519

VALUE 'CDD'.

00520

VALUE 'JES'.

00521

value 'fug'.

00522

88 L-A-CNTY-FLOOD-CNTL-DIST

VALUE 'GOC'.

00523

VALUe 'HVL'.

00524

88 LEROY-CRANDALL-AND-ASSOC

value 'LAF'.

00525

88 MOORE-AND-TABER

VALUe 'LCA'.

00526

88 ORANGE-CNTY-ENVIRON-MGMT-AGCY

VALUE 'MAT'.

00527

88 ORANGE-CNTY-FLOOD-CNTL-DIST

00528

88 ORANGE-CNTY-REGULATION-DVSN

VALUE 'OFC'.

00529

88 ORANGE-CNTY-WATER-DIST

88 SHELL-OIL-COMPANY

VALUE 'OFR'.

00530

VALUE 'OCW'.

00531

88 SOUTHERN-CALIF-EDISON-COMPANY

88 SOUTHERN-CALIF-GAS-COMPANY

VALUE 'SHO':

00532

00533

88 TEXACO-INC

00534

88 UNION-OIL-COMPANY-OF-CALIF

VALUE 'TEX'.

00535

88 USGS-CONSERVATION-DVSN

88 USGS-GEOLOGICAL-DVSN

value 'uno'.

00536

88 USGS-WATER-RESOURCES-DVSN

88 WOODWARD-CLYDE-CONSULT

09 PRIMARY-SOURCE-QUALFR

VALUE 'GSC'.

00537

VALUE 'GSG'.

00538

VALUE 'GSW'.

00539

00540

00541

00542

07 PRIMARY-ACTIVITY-CODE

88 BRIDGE-BORING

88 CORE-HOLE

88 ELECTRICAL-RESISTIVITY-SURVEY

88 FOUNDATION-INVESTIGATION

88 GRAVITY-SURVEY

88 MAGNETIC-SURVEY

88 OIL-AND-GAS-WELL

88 SEISMIC-SHOT-HOLE

88 SEISMIC-SURVEY

88 SOIL-SURVEY

88 SURFICIAL-GEOLOGY

88 TRENCH-INVESTIGATION

PIC XXX.

00544

00545

00546

00547

00548

00549

00550

00551

VALUE 'S'. 00552

VALUE 'A'.

88 WATER-WELL

VALUE 'T'.

00555 
*

* Note $\rightarrow *$ the value used to query the *

* NOTE $\rightarrow *$ SURFACE-SEGMENT-DATE MUST *

* NOTE $\rightarrow *$ BE CODED IN THE FORM 'CCYYMMDD' *

*

03 SURFACE-SEGMENT-DATE.

05 SURFACE-SGMT-CENTURY-AND-YEAR.

07 SURFACE-SGMT-CENTURY

07 SURFACE-SGMT-YEAR

05 SURFACE-SGMT-MONTH

05 SURFACE-SGMT-DAY
00557

00558

00559

00560

00561

00562

00563

00564

00565

PIC $X X$.

00566

00567

00568

PIC $X X$.

PIC $X X$.

*-- E N D O F - - SURFACE-DATA-MENUS -

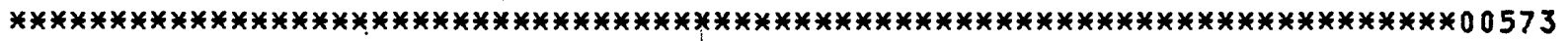

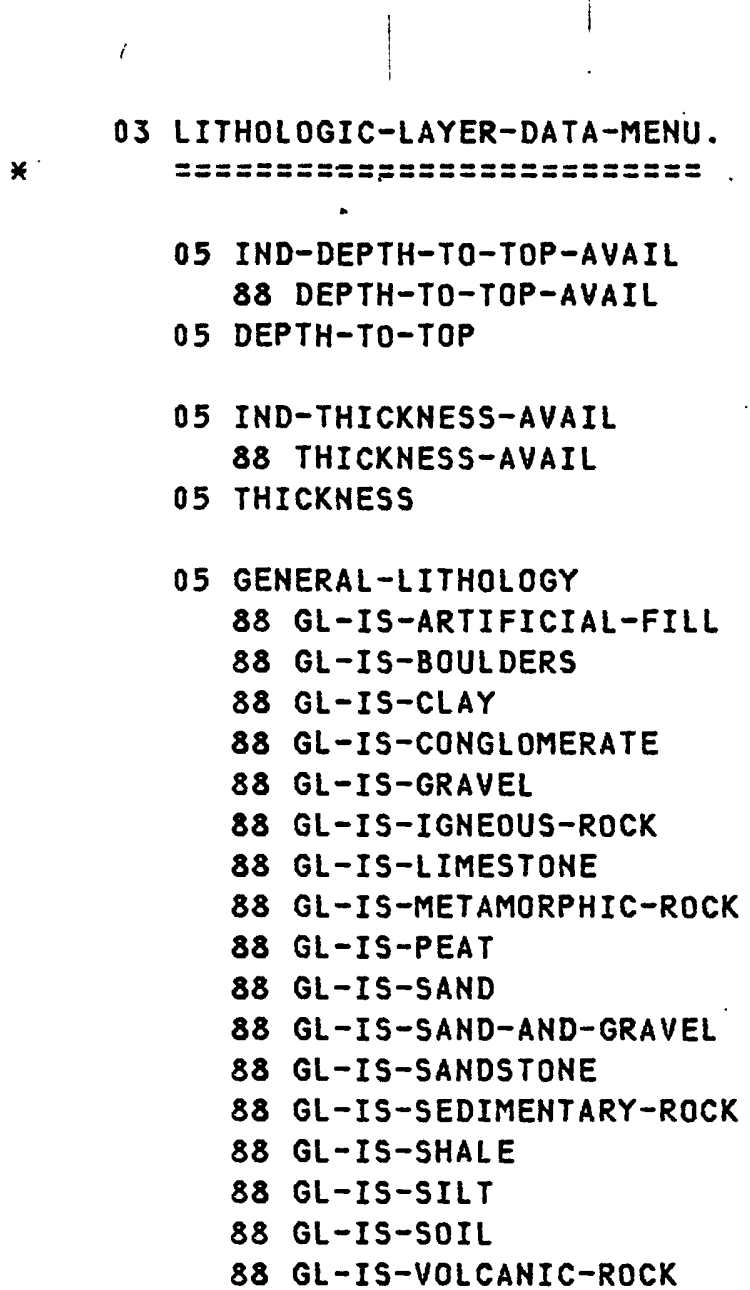

PIC $X$

VALUE. 'Y'.

00578

PIC 9(5)Y9.

00579

00580

00581

PIC $x$.

00582

VALUE ' $Y$ '. $\quad 00583$

PIC 9(5).

00584

00585

PIC $X X$.

00586

VALUE 'AF'. 00587

VALUE 'BD'. 00588

VALUE 'CL'. 00589

VALUE 'CG'. 00590

VALUE 'GR'. 00591

VALUE 'IG'. 00592

VALUE 'LS'. 00593

VALUE 'MT'. 00594

VALUE 'PT'. 00595

VALUE 'SD'. 00596

VALUE 'SG'. 00597

VALUE 'SS'. 00598

VALUE 'SR'. 00599

VALUE 'SH'. 00600

VALUE 'SL'. 00601

VALUE 'SO'. 00602

VALUE 'VO'. 00603 
05 STRATIGRAPHIC-ID.

07 STRATIGRAPHIC-GROUP

07 STRATIGRAPHIC-FORMATION

88 CAPISTRANO

88 COYOTE-HILLS-LOWER

88 COYOTE-HILLS-UPPER

88 EL-MODENO-VOLCANICS

88 FERNANDO-LOWER

88 FERNANDO-UPPER

88 LA-HABRA

88 LAKEWOOD

88 MONTEREY

88 NIGUEL

88 PICO

88 PUENTE

88 REPETTO

88 SAN-ONOFRE-BRECCIA

88 SAN-PEDRO

88 SANTIAGO

88 SESPE

88 SILVERADO

88 SUNNY-HILLS

88 TOPANGA

88 VAQUEROS

07 STRATIGRAPHIC-MEMBER

88 ALLUVIUM

88 ALPHA-AQUIFER

88 ARTESIA-AQUIFER

88 BEACH-DEPOSITS

88 BETA-AQUIFER

88 BOLSA-AQUIFER

88 COLLUVIUM

88 GAGE-AQUIFER

88 GARDENA-AQUIFER

88 GASPUR-AQUIFER

88 HOLLYDALE-AQUIFER

88 JEFFERSON-AQUIFER

88 LA-VIDA

88 LAMBDA-AQUIFER

88 LYNWOOD-AQUIFER

88 MAIN-AQUIFER

88 MEADOWLARK-AQUIFER

88 OMICRON-AQUIFER

88 RHO-AQUIFER-LOWER

88 RHO-AQUIFER-UPPER

88 SILVERADO-AQUIFER

88 SOQUEL

88 STREAM-DEPOSITS

88 SUNNYSIDE-AQUIFER

88 SYCAMORE-CANYON

88 TALBERT-AQUIFER

88 TERRACE-DEPOSITS

88 YORBA

PIC $x x$

00607

PIC XXX.

00608

VALUE '012'. 00610

VALUE '006'. 00611

VALUE '004'. 00612

VALUE $015^{\circ} .00613$

VALUE $010^{\circ} . \quad 00614$

VALUE 007 '. 00615

VALUE 002 '. 00616

VALUE '001". 00617

Value '013'. 00618

VALUE '009'. 00619

VALUE '008'. 00620

VALUE '014'. 00621

VALUE '011'. 00622

VALUE '016'. 00623

VALUE 1005 '. 00624

VALUE $1020^{\circ}$. 00625

VALUE '0191. 00626

VALUE '021'. 00627

VALUE '003'. 00628

VALUE .017'. 00629

VALUE '018'. 00630

PIC XXX. $\quad 00631$

VALUE $1001^{\prime}, \quad 00632$

VALUE 0091.00633

VALUE '012'. 00634

VALUE '003'. 00635

VALUE '010'. 00636

VALUE $.006^{\circ} . \quad 00637$

VALUE .002 '. 00638

VALUE '014'. 00639

VALUE '013'. 00640

VALUE '007'. 00641

VALUE '020'. 00642

VALUE '021'. 00643

Value '028'. 00644

VALUE '011'. 00645

VALUE '022'. 00646

VALUE '016'. 00647

VALUE '015'. 00648

VALUE $017 \%$. 00649

VALUE '019'. 00650

VALUE '018'. 00651

VALUE '023'. 00652 .

VALUE '027'. 00653

VALUE '004'. 00654

VALUE '024'. 00655

VALUE '025'. 00656

VALUE $005^{\circ}$. $\quad 00657$

VALUE '008'. 00658

VALUE '026'. 00659 
05 RELATIVE-GEOLOGIC-AGE.

07 RELATIVE-GEOLOGIC-ERA

88 CENOZOIC

88 MESOZOIC

88 PALEOZOIC

88 PRECAMBRIAN

07 RELATIVE-GEOLOGIC-PERIOD

88 CAMBRIAN

88 CRETACEOUS

88 DEVONIAN

88 JURASSIC

88 MISSISSIPPIAN

88 ORDOVICIAN

88 PENNSYLVANIAN

88 PERMIAN

88 QUATERNARY

88 SILURIAN

88 TERTIARY

88 TRIASSIC

07 RELATIVE-GEOLOGIC-SER-EPH

88 EOCENE

88 HOLOCENE

88 MIOCENE

88 OLIGOCENE

88 PALEOCENE

88 PLEISTOCENE.

88 PLIOCENE.

07 RELATIVE-GEOLOGIC-STAGE

88 MIOCENE-LOWER

88 MIOCENE-MIDDLE

88 MIOCENE-UPPER

88 PLEISTOCENE-LOWER

88 PLEISTOCENE-UPPER

88 PLIOCENE-UPPER

88 PLIOCENE-LOWER

88 RECENT

05 METHOD-OF-DETERMINATION

88 LITHOLOGY

88 MICROPALEONTOLOGY

88 PALEONTOLOGY

88 PALYNOLOGY

88 SUPERPOSITION

PIC XX.

PIC $x$.

00663

VALUe ' 1 '.

00664

VALUE ' 2 '.

00665

VALUE ' 3 '.

00666

VALUE $14^{\prime}$.

00667

PIC XX.

00668

VALUE '12'. 00670

VALUE '03'. 00671

VALUE '09'. 00672

VALUE '04'. 00673

VALUE '08'. $\quad 00674$

VALUE '11'. 00675

VALUE '07'. 00676

VALUE $06^{\prime} . \quad 00677$

VALUE '01'. 00678

VALUE '10'. 00679

VALUE '02'. 00680

VALUE $105^{\circ}, \quad 00681$

00682

VALUE '06'. 00683

VALUE '01'. 00684

VALUE '04'. 00685

VALUE '05'. 00686

VALUE '07'. 00687

VALUE '02'. 00688

VALUE $: 03$ '. 00689

PIC XXX. 00690

VALUe '008'. 00691

VALUE '007'. 00692

VALUE '006'. 00693

VALUE '003'. 00694

VALUE '002'. 00695

VALUE '004'. 00696

VALUE '005'. 00697

Value '001'. 00698

00699

PIC $X . \quad 00700$

VALUe '5'. 00701

VALUE ' 1 '. $\quad 00702$

VALUE ' 2 '. $\quad 00703$

VALUE ' 3 '. $\quad 00704$

VALUE ' 4 '. 00705 
E 15

*

* Note - $\rightarrow *$ the Value used to query the *

* NOTE - $>*$ LAYER-SEgMENT-DATE MUST *

* NOTE $\rightarrow *$ BE CODED IN THE FORM 'CCYYMMDD'*

*

03 LAYER-SEGMENT-DATE.

*

05 LAYER-SGMT-CENTURY-AND-YEAR.

07 LAYER-SGMT-CENTURY

07 LAYER-SGMT-YEAR

05 LAYER-SGMT-MONTH

05 LAYER-SGMT-DAY
00709

00710

00711

00712

00713

00714

00715

00716

00717

00718

00719

00720

00721 


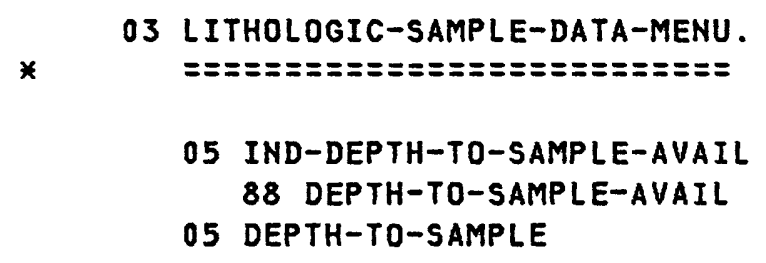

3 LITHOLOGIC-SAMPLE-DATA-MENU. 00725 00726

05 IND-DEPTH-TO-SAMPLE-AVAIL

05 DEPTH-TO-SAMPLE

PIC $x$. 00728 VALUE 'Y'. $\quad 00729$ PIC 9(5)V9. $\quad 00730$

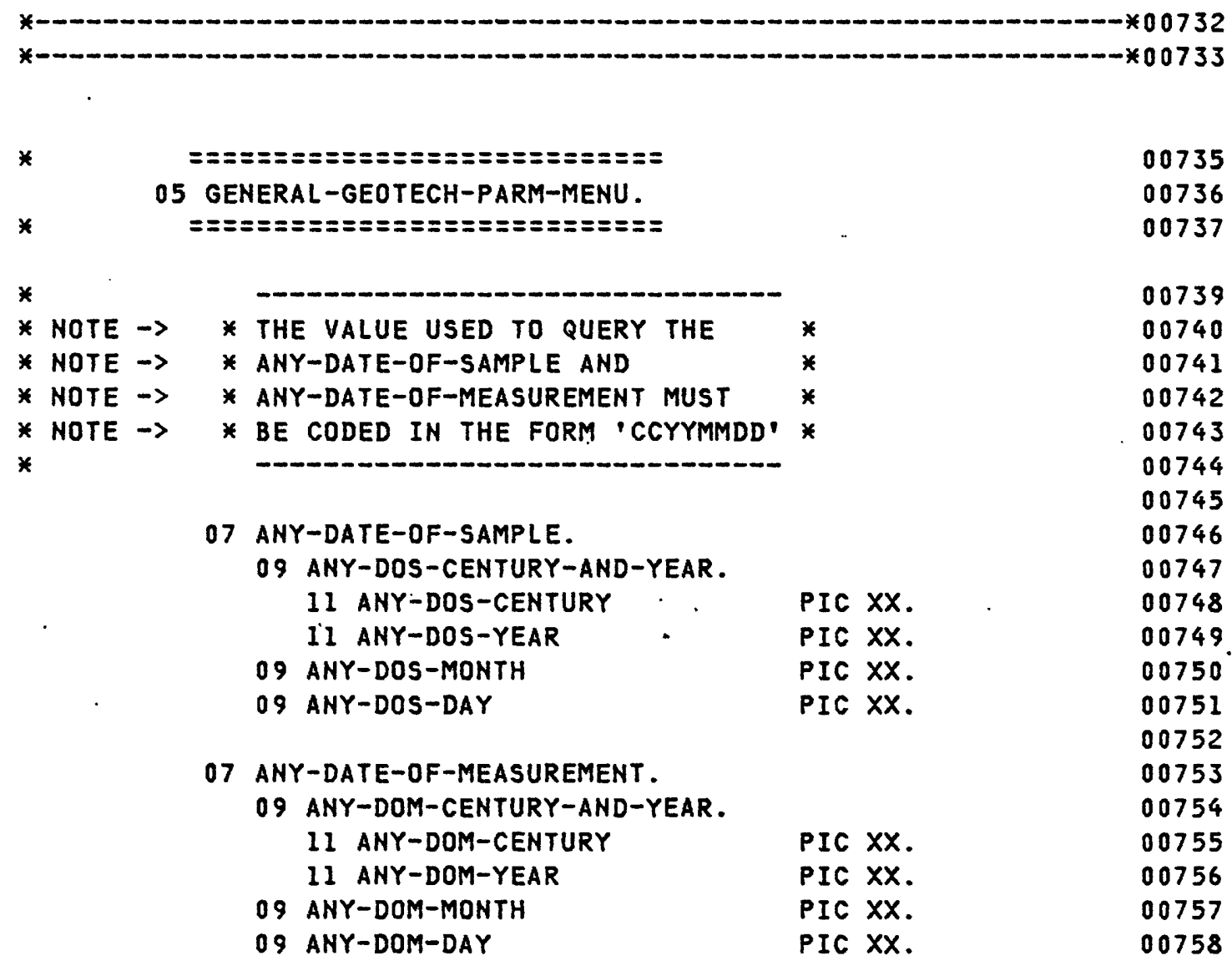

*--- GENERAL-GEOTECH-PARAMETER-MENU -- I S C ONT I N U E D - -00760 
07 ANY-METHOD

88 ANY-ACOUSTICAL-MEASUREMENT
88 ANY-AMINO-ACID
88 ANY-CALCULATED
88 ANY-CARBON-I4
88 ANY-CROSSHOLE-MEASUREMENT
88 ANY-DENDROCHRONOLOGY
88 ANY-DIRECT-OBSERVATION
88 ANY-DOWN-HOLE-MEASUREMENT
88 ANY-FISSION-TRACK
88 ANY-IN-SITU-DETERMINATION
88 ANY-INSTRUMENT-MEASUREMENT
88 ANY-LABORATORY-DETERMINATION
88 ANY-OBSIDIAN-HYDRATION
88 ANY-OIL-GAS-WELL-VELOCITY-SRVY
88 ANY-OPEN-END-TEST
88 ANY-PACKER-TEST
88 ANY-PALEOMAGNETISM
88 ANY-POTASSIUM-ARGON
88 ANY-RATIO
88 ANY-REFLECTION-MEASUREMENT
88 ANY-REFRACTION-MEASUREMENT
88 ANY-RESISTIVITY
88 ANY-SEISMIC-REFLECTION
88 ANY-SONIC-MEASUREMENT
88 ANY-STANDARD-MEASUREMENT
88 ANY-STRONTIUM-RUBIDIUM
88 ANY-TEPHRACHRONOLOGY
88 ANY-UP-HOLE-MEASUREMENT
88 ANY-URANIUM-LEAD
88 ANY-WELL-PERMEAMETER

PIC XX. 00764 00765

VALUE 'VD'. 00766 VALUE 'AG'. 00767 VALUE ' $C A$ '. 00768 VALUE 'AA'. 00769 VALUE 'VE'. 00770 VALUE 'AJ'. 00771 VALUE 'DO'. 00772 VALUE 'VB'. 00773 Value 'AE'. $\quad 00774$ VALUE 'IS'. 00775 VALUE 'IM'. 00776 VALUE 'LD'. 00777 VALUE 'AH'. 00778 VALUE 'VF'. 00779 VALUE 'OE'. 00780 VALUE 'PT'. 00781 VALUE 'AK'. 00782 VALUE 'AB'. 00783 VALUE 'RO'. 00784 VALUE 'VH'. $\quad 00785$ VALUE 'VG'. 00786 VALUE 'RE'. 00787 VALUE 'SR'. 00788 VALUE 'VC'. 00789 VALUE 'ST'. 00790 VALUE 'AC'. 00791 VALUE 'AF'. 00792 VALUE 'VA'. 00793 VALUE 'AD'. 00794 VALUE 'WP'. 00795

*--- GENERAL-GEOTECH-PARAMETER-MENU -- I S C ONT I N U E D ---00797 
*--- GENERAL-GEOTECH-PARAMETER-MENU -- C O N T I N U A T I O N---00799

07 ANY-SECONDARY-DATA-SOURCE.

00801

09 ANY-SECONDARY-DATA-SOURCE-ID.

00802

11 ANY-SECONDARY-SOURCE-CODE PIC XXX.

00803

00804

88 ANY-BEACH-LEIGHTON-AND-ASSOC

VALUE 'BLA'.

00805

88 ANY-BECHTEL-INC

VALUE 'BEC'.

00806

88 ANY-CALIF-DEPT-OF-TRANSPRTN

VALUe 'CDT'.

00807

88 ANY-CALIF-DEPT-OF-WTR-RESORCS

VALUE 'DWR'.

00808

88 ANY-CALIF-DVSN-OF-MINES--GEOL

VALUE 'DMG'.

00809

88 ANY-CALIF-DVSN-OF-OIL--GAS

VALUE 'DOG'.

00810

88 ANY-CALIF-OFFICE-OF-ARCH--CNST

88 ANY-CALIF-STATE-LANDS-CMSN

VALUE 'OAC'.

00811

00812

88 ANY-CHEVRON-USA-INC

88 ANY-CONVERSE-DAVIS-DIXON .

VALUe 'slc'.

00813

VALUE 'CHV'. 00814

88 ANY-ENGINEERING-GEOL-CONSULT

VALUE 'CDD'.

00815

value 'Jes'.

00816

88 ANY-FUGRO-INC

88 ANY-GETTY-OIL-COMPANY

value 'Fug'.

00817

VALUE 'GOC'. 00818

88 ANY-H-V-L AWMASTER-AND-CO-INC

value 'HVL'.

00819

88 ANY-L-A-CNTY-FLOOD-CNTL-DIST

VALUE 'LAF'.

00820

VALUe 'LCA'. 00821

88 ANY-LEROY-CRANDALL-AND-ASSOC

Value 'mat'.

00822

88 ANY-ORANGE-CNTY-ENVRN-MGT-AGY.

VALUE 'OCG'.

00823

88 ANY-ORANGE-CNTY-FLOOD-CTL-DST-

88 ANY-ORANGE-CNTY-REG-DVSN

value 'ofc'.

00824

VALUE 'OFR'. 00825

88 ANY-ORANGE-CNTY-WATER-DIST

VALUE 'OCW'.

00826

VALUE 'SHO'. 00827

88 ANY-SHELL-OIL-COMPANY

VALUE 'SCE'.

00828

88 ANY-SOUTHERN-CALIF-EDISON-CO

VALUE 'SCG'.

00829

88 ANY-TEXACO-INC

VALUE 'TEX'.

00830

88 ANY-UNION-OIL-COMPANY-OF-CA

VALUE 'UNO'.

00831

88 ANY-USGS-CONSERVATION-DVSN

VALUE 'GSC'.

00832

88 ANY-USGS-GEOLOGICAL-DVSN

VALUE 'GSG'.

00833

VALUE 'GSW'.

00834

88 ANY-WOODWARD-CLYDE-CONSULT

VALUE 'WCC'.

00835

00836

II ANY-SECONDARY-SOURCE-QUALFR

00837

PIC XXX.

00838

*-- GENERAL-GEOTECH-PARAMETER-MENU -- I S C O N T I N U E D---00840 
09 ANY-SECONDARY-ACTIVITY-CODE PIC $X$. 00844

88 ANY-ELEC-RESISTIVITY-SURVEY VALUE 'R'.

88 ANY-FOUNDATION-INVESTIGATION VALUE ' $F$ '.

88 ANY-GRAVITY-SURVEY

88 ANY-MAGNETIC-SURVEY

VAlUe ' $G$ '. 00850

88 ANY-OIL-AND-GAS-WELL

VALUE ' $M$ '.

00851

88 ANY-SEISMIC-SHOT-HOLE

VALUE 'O'.

00852

88 ANY-SEISMIC-SURVEY

88 ANY-SOIL-SURVEY

88 ANY-SURFICIAL-GEOLDGY

VALUE 'H".

00853

88 ANY-TRENCH-INVESTIGATION

88 ANY-WATER-WELL

VALUE ' $V$ '.

00854

VALUE ' $S$ '. 00855

VALUE 'A'. 00856

VALUE 'T". $T$ ". 00857

VALUE 'W'. 00858

07 ANY-DMG-INTERPRETED-CODE

88 ANY-DMG-INTERPRETED-VALUE

PIC $x$.

value ' $Y$ '.

00860

00861

*-D- E N D

O F - - GENERAL-GEOTECH-PARAMETER-MENU 
*

※

*

* NOTE $\rightarrow$

* NOTE $\rightarrow$

*

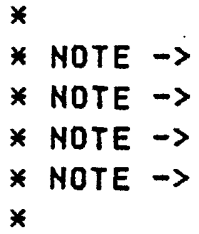

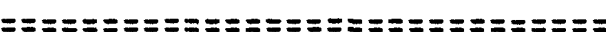

05 SPECIFIC-GEOTECH-PARM-MENUS.

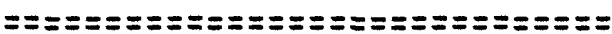

07 ABSOLUTE-GEOL-AGE-MENU.
00865

00866

00867

00869

00870

03871

00872

00873

00874

00875

00876

00877

00878

00879 .

00880

00881

11 IND-AGA-VALUE-AVAIL 88 AGA-VALUE-AVAIL

PIC $x$.

00882

00883

11 AGA-VALUE. 13 AGA-AGE

13 AGA-UNCERTAINTY

PIC $9(10)$.

00884

00885

00886

00887

13 AGA-MINIMUM-VALUE

PIC 9(10).

00888

13 AGA-MAXIMUM-VALUE

PIC 9(10).

00889

00890

00891

00892

00893

00894

00895

00896

00897

00898

00899

00900

00901

00902

00903

00904

00905

00906

00907

00908

00909

00910

00911 
*-- ABSOLUTE-GEOL-AGE-DATA-MENU - - C O N T I N U A T I 0 N - - 00915

11 AGA-METHOD

88 AGA-AMINO-ACID

88 AGA-CARBON-14

88 AGA-DENDROCHRONOLOGY

88 AGA-FISSION-TRACK

88 AGA-OBSIDIAN-HYDRATION

88 AGA-PALEOMAGNETISM

88 AGA-POTASSIUM-ARGON

88 AGA-STRONTIUM-RUBIDIUM

88 AGA-TEPHRACHRONOLOGY

88 AGA-URANIUM-LEAD
PIC XX.

00917

VALUE 'AG'. 00918

VALUE 'AA'. 00919

VALUE 'AJ'. 00920

VALUE 'AE'. 00921

VALUE 'AH'. 00922

VALUE 'AK'. 00923

VALUE 'AB'. 00924

VALUE 'AC'. 00925

VALUE 'AF'. 00926

VALUE 'AD'. 00927

00928

00929

11 AGA-SECONDARY-DATA-SOURCE.

13 AGA-SECONDARY-DATA-SOURCE-ID.

15 AGA-SECONDARY-SOURCE-CODE

PIC XXX.

15 AGA-SECONDARY-SOURCE-QUALFR

PIC XXX.

13 AGA-SECONDARY-ACTIVITY-CODE

PIC $X$.

11 AGA-DMG-INTERPRETED-CODE PIC $X$.
00930

00931

00932

00933

00934

00935

00936

00937

00938 
07 APRNT-SPEC-GRAVITY-MENU. 00940

09 IND-APRNT-SPEC-GRAVITY-AVAIL PIC $X . \quad 00943$

88 APRNT-SPEC-GRAVITY-AVAIL VALUE ' $Y$ '. 00944

09 APRNT-SPEC-GRAVITY-DATA-MENU.

11 IND-ASG-VALUE-AVAIL 88 ASG-VALUE-AVAIL

PIC $X$.

00945

00946

II ASG-VALUE PIC 99V99. 00949

VALUe 'Y'.

00947

00948

00950

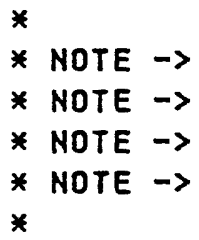

* the value used to query the

00951

00952

* ASG-DATE-OF-SAMPLE AND *

00953

* ASG-DATE-DF-MEASUREMENT MUST * $\quad$ 00954

* BE CODED IN THE FORM 'CCYYMMDD' * 00955

00956

0.0957

11 ASG-DATE-DF-SAMPLE.

00958

13 ASG-DOS-CENTURY-AND-YEAR.
15 ASG-DOS-CENTURY
PIC $X X$.
15 ASG-DOS-YEAR
PIC $X X$.
13 ASG-DOS-MONTH
PIC $X X$.
13 ASG-DOS-DAY
PIC $X X$.

00959

00960

00961

00962

00963

00964

11 ASG-DATE-OF-MEASUREMENT.

00965 .

13 ASG-DOM-CENTURY-AND-YEAR .
15 ASG-DOM-CENTURY
PIC $X X$. 15 ASG-DOM-YEAR.
PIC $X X$.
13 ASG-DOM-MONTH
PIC $X X$.
13 ASG-DOM-DAY
PIC $X X$.

00966 .

00967

00968

00969

00970

00971

11 ASG-METHOD 88 ASG-RATIO

PIC $X X$.

00972

00973

00974

11 ASG-SECONDARY-DATA-SOURCE.

00975

13 ASG-SECONDARY-DATA-SOURCE-ID.

00976

15 ASG-SECONDARY-SOURCE-CODE

PIC XXX.

00977

00978

15 ASG-SECONDARY-SOURCE-QUALFR

00979

00980

13 ASG-SECONDARY-ACTIVITY-CODE PIC XXX.

PIC $X$.

11 ASG-DMG-INTERPRETED-CODE PIC $X$. 


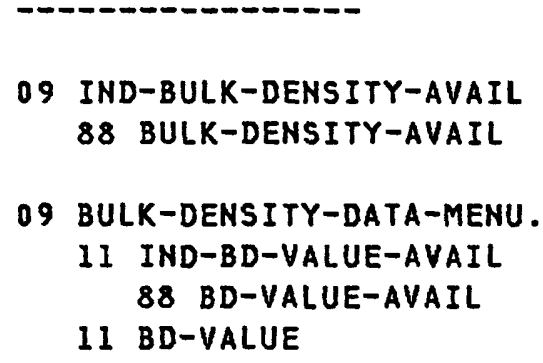

09 IND-BULK-DENSITY-AVAIL 88 BULK-DENSITY-AVAIL

09 BULK-DENSITY-DATA-MENU.

11 IND-BD-VALUE-AVAIL 88 BD-VALUE-AVAIL

11 BD-VALUE

PIC $X$.

VALUE ' $Y$ '.

00990

00991

00992

00993

00994

PIC $X$.

00995

VALUE ' $Y$ '.

00996

00997

PIC 999V9.

00998

00999

01000

* NOTE $\rightarrow$

* NOTE $\rightarrow$

* NOTE $\rightarrow$

* NOTE $\rightarrow$

*
* the value used to query the
* BD-DATE-OF-SAMPLE AND
* BD-DATE-OF-MEASUREMENT MUST *
* be CODED IN THE FORM 'CCYYMMDd' *

01001

01002

01003

01004

01005

01006

11 BD-DATE-OF-SAMPLE.

01007

13 BD-DOM-CENTURY-AND-YEAR.

$\begin{array}{ll}15 \text { BD-DOM-CENTURY } & \text { PIC } X X . \\ 15 \text { BD-DOM-YEAR } & \text { PIC } X X . \\ \text { BD-DOM-MONTH } & \text { PIC XX. } \\ \text { BD-DOM-DAY } & \text { PIC XX. }\end{array}$

01008

01009

01010

01011

01012

01013

11 BD-DATE-OF-MEASUREMENT.

01014

13 BD-DOM-CENTURY-AND-YEAR. $\begin{array}{ll}15 \text { BD-DOM-CENTURY } & \text { PIC } X X . \\ 15 \text { BD-DOM-YEAR } & \text { PIC XX. } \\ \text { BD-DOM-MONTH } & \text { PIC } X X . \\ \text { BD-DOM-DAY } & \text { PIC } X X .\end{array}$

01015

01016

01017

01018

01019

01020

11 BD-METHOD

PIC XX.

01021

88 BD-STANDARD-MEASUREMENT VALUE 'ST'. 01022

11 BD-SECONDARY-DATA-SOURCE.

13 BD-SECONDARY-DATA-SOURCE-ID.

01023

01024

01025

15 BD-SECONDARY-SOURCE-CODE

PIC $X X X$.

01026

01027

15 BD-SECONDARY-SOURCE-QUALFR

01028

01029

13 BD-SECONDARY-ACTIVITY-CODE

01030

01031

01032

11 BD-DMG-INTERPRETED-CODE PIC $X$. 01033 
07 BULK-MODULUS-MENU. . . 01035

09 IND-BULK-MODULUS-AVAIL 88 BULK-MODULUS-AVAIL

PIC $X$.

01038

VALUE 'Y'. $\quad 01039$

01040

09 BULK-MODULUS-DATA-MENU.

11 IND-BM-VALUE-AVAIL 88 BM-VALUE-AVAIL

PIC $X$.

01041

11 BM-VALUE

VALUE ' $Y$ '.

01042

01043

PIC 9999.

*

* NOTE $\rightarrow$

* NOTE $\rightarrow$

* NOTE $\rightarrow$

* NOTE $\rightarrow$
䒔
* the value used to query the *

* BM-DATE-OF-SAMPLE AND *

* BM-DATE-OF-MEASUREMENT MUST *

* BE CODED IN THE FORM 'CCYYMMDD' *

11 BM-DATE-OF-SAMPLE.

13 BM-DOS-CENTURY-AND-YEAR.
15 BM-DOS-CENTURY
PIC $X X$.
15 BM-DOS-YEAR
PIC $X X$.
13 BM-DOS-MONTH
PIC $X X$.
PIC $X X$.

13 BM-DOS-DAY

01044

01045

01046

01047

01048

01049

01050

01051

01052

01053

01054

01055

01056

01057

01058

01059

01060

01061

01062

01063

01064

01065

01066

11 BM-METHOD

PIC XX.

01067

88 BM-CALCULATED

VALUe ' $C A$ '.

01068

88 BM-IN-SITU-DETERMINATION VALUE 'IS'.

88 BM-LABORATORY-DETERMINATION

value 'LD'.

01069

01070

01071

01072

01073

11 BM-SECONDARY-DATA-SOURCE.

01074

13 BM-SECONDARY-DATA-SOURCE-ID. 15 BM-SECONDARY-SOURCE-CODE

PIC XXX.

01075

01076

15 BM-SECONDARY-SOURCE-QUALFR

01077

01078

13 BM-SECONDARY-ACTIVITY-CODE

01079

PIC $X$.

11 BM-DMG-INTERPRETED-CODE PIC $x$. 
07 GRAIN-SIZE-MENU. $\quad 01084$

*

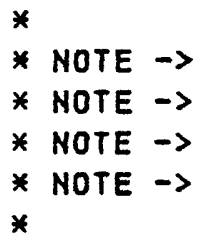

09 IND-GRAIN-SIZE-AVAIL 88 GRAIN-SIZE-AVAIL

09 GRAIN-SIZE-DATA-MENU.

11 GS-VALUE.

13 IND-GS-004-VALUE-AVAIL PIC $X$. 88 GS-004-VALUE-AVAIL

13 GS-004-VALUE VALUE 'Y'. PIC 999.

13 IND-GS-010-VALUE-AVAIL PIC $x$. 88 GS-0IO-VALUE-AVAIL

13 GS-OIO-VALUE

13 IND-GS-040-VALUE-AVAIL PIC $x$. value 'y'. PIC 999.

PIC $X$.

VALUe 'Y'.

01085

01086

01087

01088

01089

01090

01091

01092

01093

01094

01095

01096

01097

01098

01099

13 GS-040-VALUE

PIC 999.

value 'y'.

01100

01101

01102

01103

01104

01105

01106

01107

01108

01109

01110

01111

01112

01113

01114

01115

01116

01117

01118

01119

01120

01121

01122

01123

01124

01125

01126

11 GS-METHOD

PIC $X X$.

88 GS-LABORATORY-DETERMINATION

VALUe 'LD'.

11 GS-SECONDARY-DATA-SOURCE.

13 GS-SECONDARY-DATA-SOURCE-ID.

15 GS-SECONDARY-SOURCE-CODE

PIC XXX.

15 GS-SECONDARY-SOURCE-QUALFR

PIC XXX.

13 GS-SECONDARY-ACTIVITY-CODE

PIC $X$.

01127

01128

01129

01130

01131

01132

01133

01134

01135

01136

01137

01138

11 GS-DMG-INTERPRETED-CODE PIC $X$. 


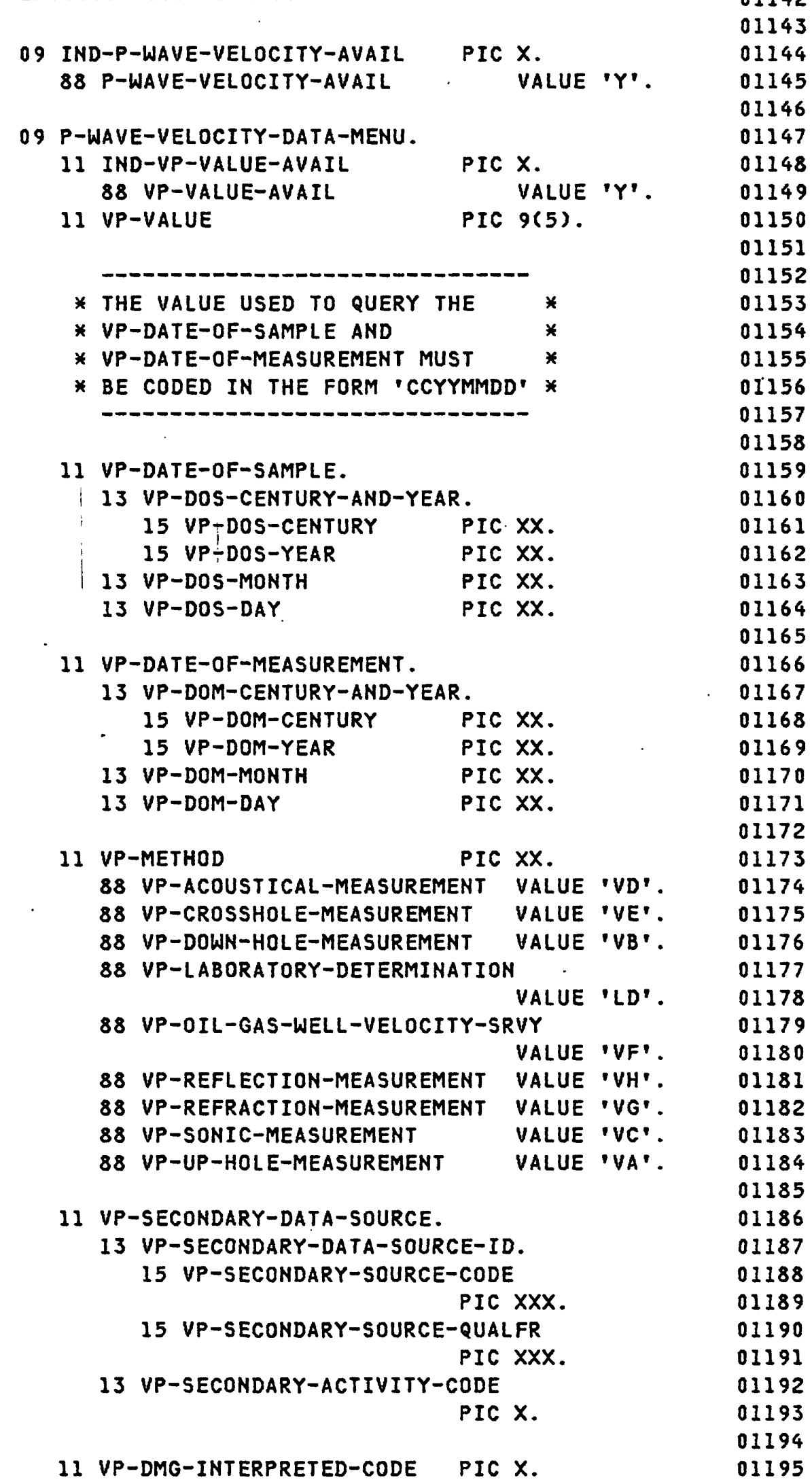

*

* NOTE $\rightarrow$

* NOTE $\rightarrow$

* NOTE $\rightarrow$

* NOTE $\rightarrow$

*
01141

01143

01144

01145

01146

01147

01148

01150

01151

01152

01154

01155

01156

01157

01158

01159

01160

01161

01162

01164

01165

01166

01168

01169

01170

01171

01172

01173

01174

01175

01176

01178

01179

01180

01182

01183

01184

01185

01186

01187

01189

01190

01191

01193

01195 
09 IND-PERMEABILITY-AVAIL

88 PERMEABILITY-AVAIL

PIC $x$.

01199

value 'y'.

01200

09 PERMEABILITY-DATA-MENU.

11 IND-PERM-VALUE-AVAIL 88 PERM-VALUE-AVAIL

11 PERM-VALUE

PIC $x$.

01201

01202

01203

01204

Value ' $Y$ '. 01205

PIC $9(5) . \quad 01206$

01207

01208

* the value used to query the *

01209

NOTE $\rightarrow$

NOTE $\rightarrow$

* NOTE $\rightarrow$

NOTE $\rightarrow$

* PERM-DATE-OF-SAMPLE AND *

* PERM-DATE-OF-MEASUREMENT MUST *

01210

*

* BE CODED IN THE FORM 'CCYYMMDD' *

01212

01213

01214

11 PERM-DATE-OF-SAMPLE.

13 PERM-DOS-CENTURY-AND-YEAR. 15 PERM-DOS-CENTURY PIC $X X$. 15 PERM-DOS-YEAR PIC $X X$.

01215

01216

01217

01218

13 PERM-DOS-MONTH

PIC $X X$.

01219

13 PERM-DOS-DAY

PIC $X X$.

01220

01221

11 PERM-DATE-OF-MEASUREMENT.

13 PERM-DOM-CENTURY-AND-YEAR.

01222

01223

15 PERM-DOM-CENTURY PIC $X X$.

01224

15 PERM-DOM-YEAR

PIC $X X$.

01225

13 PERM-DOM-MONTH

PIC $X X$.

01226

13 PERM-DOM-DAY

PIC XX.

01227

11 PERM-METHOD

PIC XX.

01228

01229

88 PERM-LABORATORY-DETERMINATION

01230

88 PERM-OPEN-END-TEST

VALUE 'LD'. 01231

88 PERM-PACKER-TEST

VALUE 'OE'.

01232

88 PERM-WELL-PERMEAMETER

VALUE 'PT'.

01233

VALUE 'WP'.

01234

01235

11 PERM-SECONDARY-DATA-SOURCE.

01236

13 PERM-SECONDARY-SOURCE-IN.

15 PERM-SECONDARY-SOURCE-CODE

01237

PIC XXX.

01238

15 PERM-SECONDARY-SOURCE-QUALFR

01239

01240

PIC XXX.

01241

13 PERM-SECONDARY-ACTIVITY-CODE

01242

PIC $X$.

01243

01244

11 PERM-DMG-INTERPRETED-CODE PIC $x$.

01245 
*

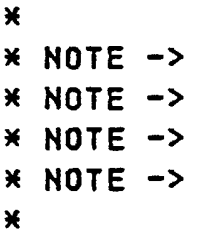

07 POISSONS-RATIO-MENU.

01247

01248

01249

01250

09 IND-POISSONS-RATIO-AVAIL 88 POISSONS-RATIO-AVAIL

PIC $x$.

value ' $y$ '.

01251

01252

01253

09 POISSONS-RATIO-DATA-MENU.
11 IND-PR-VALUE-AVAIL 88 PR-VALUE-AVAIL
11 PR-VALUE

PIC $X$.

VALUE 'Y'. 01255

PIC V99. 01256

01257

01258

01259

01260

01261

01262

01263

01264

01265

11 PR-DATE-OF-SAMPLE.

13 PR-DOS-CENTURY-AND-YEAR.

15 PR-DOS-CENTURY

15 PR-DOS-YEAR

PIC $X X$.

PIC $X X$.

PIC $X X$.

13 PR-DOS-MONTH

13 PR-DOS-DAY

PIC $X X$.

11 PR-DATE-OF-MEASUREMENT.

13 PR-DOM-CENTURY-AND-YEAR.

15 PR-DOM-CENTURY

15 PR-DOM-YEAR

13 PR-DOM-MONTH

13 PR-DOM-DAY

PIC $X X$.
PIC $X X$.

PIC $X X$.

PIC $X X$.

01266

01267

01268

01269

01270

01271

01272

01273

01274

0127.5

01276

01277

01278

11 PR-METHOD

PIC $X X$.

01279

88 PR-CALCULATED

Value ' $C A$ '.

01280

88 PR-IN-SITU-DETERMINATION

VALUe 'IS'.

01281

88 PR-LABORATORY-DETERMINATION

VALUe 'LD'. . 01283

01284

01285

01286

01287

01288

01289

01290

13 PR-SECONDARY-ACTIVITY-CODE

PIC XXX.

01291

01292

01293

11 PR-DMG-INTERPRETED-CODE PIC $x$.

01294 
09 IND-POROSITY-AVAIL 88 POROSITY-AVAIL

09 POROSITY-DATA-MENU.

II IND-POR-VALUE-AVAIL 88 POR-VALUE-AVAIL

11 POR-VALUE
PIC $x$.

value ' $Y$ '.

01297

01298

01299

01300

01301

01302

01303

01304

01305

01306

01307

01308

01309

01310

01311

01312

01313

01314

01315

01316

01317

01318

01319

01320

01321

01322

01323

01324

01325

01326

01327

01328

01329

01330

01331

01332

01333

01334

01335

01336

01337

01338

01339

01340 
产

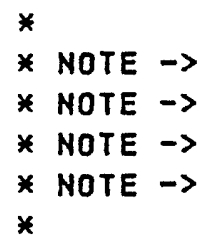

07 RELATIVE-DENSITY-MENU.

01342

01343

01344

09 IND-RELATIVE-DENSITY-AVAIL 88 RELATIVE-DENSITY-AVAIL

PIC $x$.

01345

01346

01347

01348

09 RELATIVE-DENSITY-DATA-MENU.

11 IND-RD-VALUE-AVAIL 88 RD-VALUE-AVAIL

11 RD-VALUE

PIC $x$.

VALUE ' $Y$ '. $\quad 01350$

PIC 99.

01351

01352

01353

01354

01355

01356

01357

01358

01359

01360

01361

01362

01363

01364

01365

01366

01367

01368

01369

01370

01371

01372

01373

11 RD-METHOD

88 RD-RATIO

PIC $X X$.

01374

01375

01376

01377

01378

01379

01380

01381

01382

01383

13 RD-SECONDARY-ACTIVITY-CODE

PIC $X$.

01384

01385

11 RD-DMG-INTERPRETED-CODE PIC $x$. 
09 IND-S-WAVE-VELOCITY-AVAIL 88 5-WAVE-VELOCITY-AVAIL

PIC $x$. VALUE ' $Y$ '.

09 5-WAVE-VELOCITY-DATA-MENU. 11 IND-VS-VALUE-AVAIL 88 US-VALUE-AVAIL

11 VS-VALUE
PIC $x$.

VAlUe ' $Y$ '.

PIC 9(5).
*

NOTE $\rightarrow$

NOTE $\rightarrow$

NOTE $\rightarrow$
* the value used to query the

* VS-DATE-DF-MEASUREMENT MUST *

* BE CODED IN THE FORM 'CCYYMMDd' *

11 VS-DATE-OF-SAMPLE.

13 VS-DOS-CENTURY-AND-YEAR.

$\begin{array}{ll}15 \text { US-DOS-CENTURY } & \text { PIC XX. } \\ 15 \text { VS-DOS-YEAR } & \text { PIC XX. } \\ \text { US-DOS-MONTH } & \text { PIC XX. } \\ \text { US-DOS-DAY } & \text { PIC XX. }\end{array}$

11 VS-DATE-OF-MEASUREMENT .

13 VS-DOM-CENTURY-AND-YEAR.
15 VS-DOM-CENTURY
PIC $X X$..
15 US-DOM-YEAR
PIC $X X$.
13 VS-DOM-MONTH
PIC $X X$.
PIC $X X$.

13 VS-DOM-DAY

PIC $X X$.

11 VS-METHOD

88 VS-ACOUSTICAL-MEASUREMENT

88 VS-CROSSHOLE-MEASUREMENT

VALUE 'VD'.

VALUE 'VE'.

88 VS-DOWN-HOLE-MEASUREMENT VALUE 'VB'.

88 VS-LABORATORY-DETERMINATION

88 VS-OIL-GAS-WELL-VELOCITY-SRVY

VALUE 'LD'.

$\begin{array}{lll}88 & \text { VS-REFLECTION-MEASUREMENT } & \text { VALUE 'VAL'. } \\ 88 \text { VS-REFRACTION-MEASUREMENT } & \text { VALUE 'VG'. } \\ 88 \text { VS-SONIC-MEASUREMENT } & \text { VALUE 'VC'. } \\ 88 \text { VS-UP-HOLE-MEASUREMENT } & \text { VALUE 'VA'. }\end{array}$

11 VS-SECONDARY-DATA-SOURCE.

13 VS-SECONDARY-DATA-SOURCE-ID.

15 US-SECONDARY-SOURCE-CODE

PIC XXX.

15 VS-SECONDARY-SOURCE-QUALFR

PIC XXX.

13 VS-SECONDARY-ACTIVITY-CODE

PIC $X$.

11 VS-DMG-INTERPRETED-CODE PIC $x$.

01399

01400

01401

01402

01403

01404

01405

01406

01407

01408

01409

01410

01411

01412

01413

01414

01415

01416

01417

01418

01419

01420

01421

01422

01423

01424

01425

01426

01427

01428

01429

01430

01431

01432

01433

01434

01435

01436

01437

01438

01439

01440

01441 
*

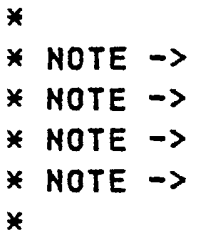

07 SEISMIC-IMPEDANCE-MENU. $\quad 01443$

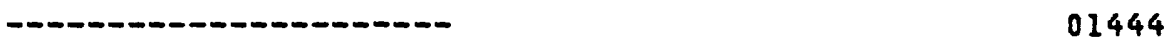
01445 01446 01447 01448 01449 01450 01451 01452 01453 01454 01455 01456 01457 01458 01459 01460 01461 01462 01463 01464 01465 01466 01467 01468 01469 01470 01471 01472 01473 01474

11 SI-METHOD 88 SI-CALCULATED

PIC $X X$. VAlUe 'CA'. 01476 01477 01478 01479 01480 01481 01482 01483 01484 01485 01486 11 SI-DMG-INTERPRETED-CODE PIC $X$. 
O7 SHEAR-MODULUS-MENU.

01489

*
09 IND-SHEAR-MODULUS-AVAIL 88 SHEAR-MODULUS-AVAIL
O9 SHEAR-MODULUS-DATA-MENU.
11 IND-SM-VALUE-AVAIL 88 SM-VALUE-AVAIL
11 SM-VALUE

PIC $x$.

value ' $y$ '.

01490

01491

01492

01493

01494

01495

PIC $x$.

01496

VALUE 'Y'. 01497

PIC 9999.

01498

01499

01500

NOTE $\rightarrow$

NOTE $\rightarrow$

NOTE $\rightarrow$

NOTE $\rightarrow$

* the value used to query the

01501

* SM-DATE-OF-SAMPLE AND *

* SM-DATE-OF-MEASUREMENT MUST *

01502

* BE CODED IN THE FORM 'CCYYMMDD' *

01503

01504

01505

01506

11 SM-DATE-OF-SAMPLE.

01507

13 SM-DOS-CENTURY-AND-YEAR .

$\begin{array}{ll}15 \text { SM-DOS-CENTURY } & \text { PIC XX. } \\ 15 \text { SM-DOS-YEAR } & \text { PIC XX. } \\ \text { SM-DOS-MONTH } & \text { PIC XX. } \\ \text { SM-DOS-DAY. } & \text { PIC XX. }\end{array}$

01508

01509

01510

01511

01512

01513

11 SM-DATE-OF-MEASUREMENT.

13 SM-DOM-CENTURY-AND-YEAR.

$\begin{array}{ll}\text { I5 SM-DOM-CENTURY } & \text { PIC XX. } \\ \text { i5 SM-DOM-YEAR } & \text { PIC XX. } \\ 13 \text { SM-DOM-MONTH } & \text { PIC XX. } \\ 13 \text { SM-DOM-DAY } & \text { PIC XX. }\end{array}$

01514

01515

01516

01517

01518

01519

01520

11 SM-METHOD

PIC $X X$

01521

88 SM-CALCULATED

Value ' $C A$ '.

01522

88 SM-IN-SITU-DETERMINATION VALUE 'IS'.

88 SM-LABORATORY-DETERMINATION

01523

01524

VAlue 'LD'。

11 SM-SECONDARY-DATA-SOURCE.

01525

01526

01527

13 SM-SECONDARY-DATA-SOURCE-ID.

15 SM-SECONDARY-SOURCE-CODE

01528

01529

01530

15 SM-SECONDARY-SOURCE-QUALFR

01531

01532

13 SM-SECONDARY-ACTIVITY-CODE

01533

01534

01535

11 SM-DMG-INTERPRETED-CODE PIC $X$.

01536 
07 SHEAR-STRENGTH-MENU. $\quad 01538$

09 IND-SHEAR-STRENGTH-AVAIL

PIC $X$.

01540 88 SHEAR-STRENGTH-AVAIL

value ' $Y$ '.

01541

01542

09 SHEAR-STRENGTH-DATA-MENU.

11 IND-SHST-VALUE-AVAIL 88 SHST-VALUE-AVAIL

PIC $X$. 01543 01544 11 SHST-VALUE VALUE ' $Y$ '. 01545 01546 PIC 9(5)V9. 01547 01548 01549 01550 01551 01552 01553 01554 01555

11 SHST-DATE-OF-SAMPLE. 01556 13 SHST-DOS-CENTURY-AND-YEAR. 15 SHST-DOS-CENTURY PIC XX. 15 SHST-DOS-YEAR PIC $X X$. 01557 01558 13 SHST-DOS-MONTH PIC $X X$. 13 SHST-DOS-DAY PIC $X X$.

01559

01560 01561 01562

11 SHST-DATE-OF-MEASUREMENT.

13 SHST-DOM-CENTURY-AND-YEAR.

01563 01564 15 SHST-DOM-CENTURY PIC XX. 15 SHST-DOM-YEAR PIC XX. 01566

13 SHST-DOM-MONTH PIC XX. 01567

13 SHST-DOM-DAY PIC XX.

11 SHST-METHOD 88 SHST-CALCULATED

PIC $X X$. 01569 01570 88 SHST-INSTRUMENT-MEASUREMENT 01571 01572 01573 01574 01575

11 SHST-SECONDARY-DATA-SOURCE. $\begin{array}{ll}13 \text { SHST-SECONDARY-DATA-SOURCE-ID. } & 01576 \\ 15 \text { SHST-SECONDARY-SOURCE-CODE } & 01577\end{array}$ PIC XXX. $\quad 01578$ 15 SHST-SECONDARY-SOURCE-QUALFR 01579 PIC XXX. $\quad 01580$ 13 SHST-SECONDARY-ACTIVITY-CODE 01581 PIC $X$. 01582 01583

11 SHST-DMG-INTERPRETED-CODE PIC $x$. 01584 
07 SPEC-SMPL-LITHOLOGY-MENU.

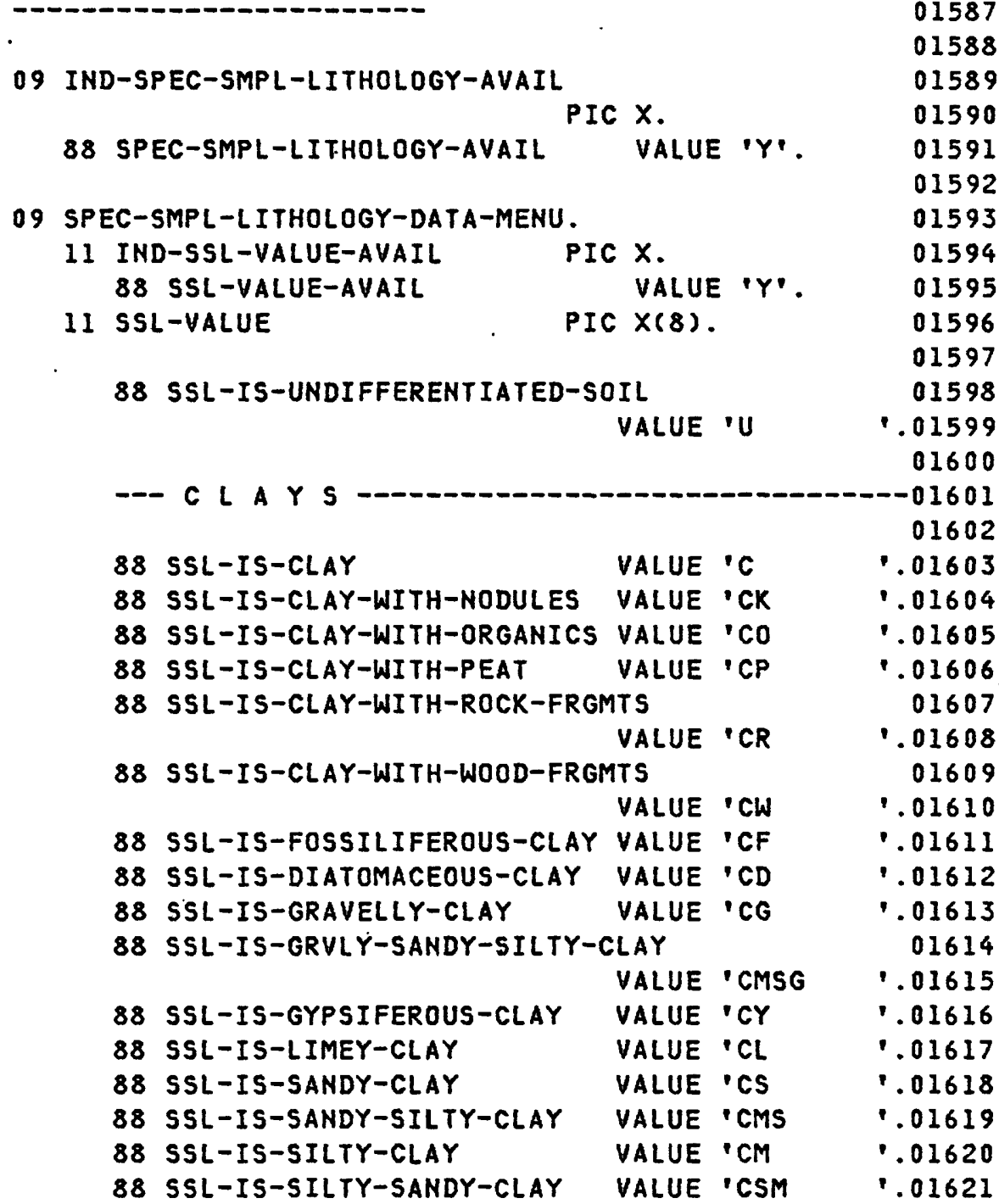


*--- SPEC-SMPL-LITHOLOGY-DATA-MENU -- C ONTI N U A T I O N ---01627

*- S S L - V A L U E - C ONT I N UAT I O N - - 01629

*

\begin{tabular}{|c|c|c|c|c|}
\hline & & & & 01632 \\
\hline 88 & SSL-IS-CLAYEY-GRAVEL & VALUE & GC & .01633 \\
\hline 88 & SSL-IS-CLAYEY-SANDY-SILTY- & -GRVL & & 01634 \\
\hline & & VALUE & ' GSMC & .01635 \\
\hline 88 & SSL-IS-GRAVEL & VALUE & 'G & .01636 \\
\hline 88 & SSL-IS-GRAVEL-WITH-CBLS-BL & LDRS & & 01637 \\
\hline & & VALUE & 'GB & .01638 \\
\hline 88 & SSL-IS-GRAVEL-WITH-ROCK-FR & RGMTS & & 01639 \\
\hline & & VALUE & 'GR & .01640 \\
\hline 88 & SSL-IS-GRAVEL-WITH-WOOD-FR & RGMTS & & 01641 \\
\hline & & VALUE & 'GW & .01642 \\
\hline 38 & SSL-IS-GYPSIFEROUS-GRAVEL & VALUE & 'GY & 1.01643 \\
\hline 88 & SSL-IS-FOSSILIFEROUS-GRAVE & & & 01644 \\
\hline & & VALUE & 'GF & '.01645 \\
\hline 88 & SSL-IS-LIMEY-GRAVEL & VALUE & 'GL & .01646 \\
\hline 88 & SSL-IS-SANDY-GRAVEL & VALUE & 'GS & .01647 \\
\hline 88 & SSL-IS-SANDY-SILTY-GRAVEL & VALUE & 'GSM & .01648 \\
\hline 88 & SSL-IS-SILICEOUS-GRAVEL & VALUE & 'GX & .01649 \\
\hline 88 & SSL-IS-SILTY-GRAVEL & VALUE & ' GM & $.01650^{\circ}$ \\
\hline
\end{tabular}

*-_- S S L - V A L UE - I S C ON T I N UE D - - 01652

*--- SPEC-SMPL-LITHOLOGY-DATA-MENU -- I S CONT INUE D --01654 
SPEC-SMPL-LITHOLOGY-DATA-MENU --- C ON T I N U A T I O N---01656

SSL- YALUE-- C ONTIN UATION---01658

*

\begin{tabular}{|c|c|c|c|c|}
\hline & & & & 01661 \\
\hline 88 & SSL-IS-CLAYEY-SAND & VALUE & 'sc & .01662 \\
\hline 8 & SSL-IS-CLAYEY-SILTY-SAND & VALUE & 'SMC & 1.01663 \\
\hline 38 & SSL-IS-FOSSILIFEROUS-SAND & VALUE & 'SF & 1.01664 \\
\hline 38 & SSL-IS-GRAVELLY-SAND & VALUE & '5G & .01665 \\
\hline 88 & SSL-IS-GRVLY-SILTY-CLAYEY- & -SAND & & 01666 \\
\hline & & VALUE & - SCMG & .01667 \\
\hline 8 & SSL-IS-GYPSIFEROUS-SAND & VALUE & 'SY & .01668 \\
\hline 38 & SSL-IS-LIMEY-SAND & VALUE & 'SL & 1.01669 \\
\hline 88 & SSL-IS-SAND & VALUE & 's & .01670 \\
\hline 88 & SSL-IS-SAND-WITH-CBLS-BLDR & & & 01671 \\
\hline & & VALUE & 'SB & .01672 \\
\hline 38 & SSL-IS-SAND-WI TH-NODULES & VALUE & 'SK & .01673 \\
\hline 38 & SSL-IS-SAND-WITH-ORGANICS & VALUE & 'so & .01674 \\
\hline 38 & SSL-IS-SAND-WITH-PEAT & VALUE & 'SP & 1.01675 \\
\hline 38 & SSL-IS-SAND-WITH-ROCK-FRGM & & & 01676 \\
\hline & & VALUE & 'SR & 1.01677 \\
\hline 38 & SSL-IS-SAND-WITH-WOOD-FRGM & & & 01678 \\
\hline & & VALUE & 'sw & 1.01679 \\
\hline 88 & SSL-IS-SANDEY-GRAVELLY-SAN & & & 01680 \\
\hline & & VALUE & 'SCG & .01681 \\
\hline 88 & SSL-IS-SILICEOUS-SAND & VALUE & 'sx & .01682 \\
\hline 38 & SSL-IS-SILTY-SAND & VALUE & 'SM & 1.01683 \\
\hline 38 & SSL-IS-SILTY-CLAYEY-SAND & VALUE & ' SCM & .01684 \\
\hline 88 & SSL-IS-SILTY-GRAVELLY-SAND & & & 01685 \\
\hline & & VALI & 'SMG & 1.01686 \\
\hline
\end{tabular}

*---- S S L - V A L UE-- I S C ONTINUED---01688

*--- SPEC-SMPL-LITHOLOGY-DATA-MENU --- I S C O N T I N U E D---01690 
*-- SPEC-SMPL-LITHOLOGY-DATA-MENU -- C 0 N T I N U A T I 0 N --01692

*----- S S L - V A L U E -- C O N T I N U A T I O N - -01694

$*$

\begin{tabular}{|c|c|c|c|c|}
\hline & & & & 01697 \\
\hline 88 & SSL-IS-CLAYEY-SANDY-SILT & VALUE & 'MSC & .01698 \\
\hline 88 & SSL-IS-CLAYEY-SILT & VALUE & 'MC & 1.01699 \\
\hline 88 & SSL-IS-FOSSILIFEROUS-SILT & VALUE & 'MF & .01700 \\
\hline 88 & SSL-IS-GRAVELLY-SILT & VALUE & 'MG & .01701 \\
\hline \multirow[t]{2}{*}{88} & \multicolumn{3}{|c|}{ SSL-IS-GRVLY-SANDY-CLAYEY-SILT } & 01702 \\
\hline & & VALUE & 'MC5G & .01703 \\
\hline 88 & SSL-IS-GYPSIFEROUS-SILT & VALUE & MY & .01704 \\
\hline 88 & SSL-IS-LIMEY-SILT & VALUE & 'ML & 1.01705 \\
\hline 88 & SSL-IS-SANDY-SILT & VALUE & 'MS & .01706 \\
\hline 88 & SSL-IS-SANDY-CLAYEY-SILT & VALUE & 'MCS & .01707 \\
\hline 88 & SSL-IS-SILICEOUS-SILT & VALUE & ' $M x$ & 1.01708 \\
\hline 88 & SSL-IS-SILT & VALUE & 'M & .01709 \\
\hline 88 & SSL-IS-SILT-WITH-NODULES & VALUE & 'MK & .01710 \\
\hline 88 & SSL-IS-SILT-WITH-ORGANICS & VALUE & 'MO & .01711 \\
\hline 88 & SSL-IS-SILT-WITH-PEAT & VALUE & 'MP & .01712 \\
\hline \multirow[t]{2}{*}{88} & \multicolumn{3}{|l|}{ SSL-IS-SILT-WITH-ROCK-FRGMTS } & 01713 \\
\hline & & VALUE & 'MR & .01714 \\
\hline \multirow[t]{2}{*}{88} & \multicolumn{3}{|l|}{ SSL-IS-SILT-WITH-WOOD-FRGMTS } & 01715 \\
\hline & & VALUE & 'MW & .01716 \\
\hline
\end{tabular}

*-- 5 L - V A L U E - I S C O N T I N U E D - - 01718

*--- SPEC-SMPL-LITHOLOGY-DATA-MENU -- I S C ON T INU E D ---01720 
*--- SPEC-SMPL-LITHOLOGY-DATA-MENU -- C O N T I N U A T I O N ---01722

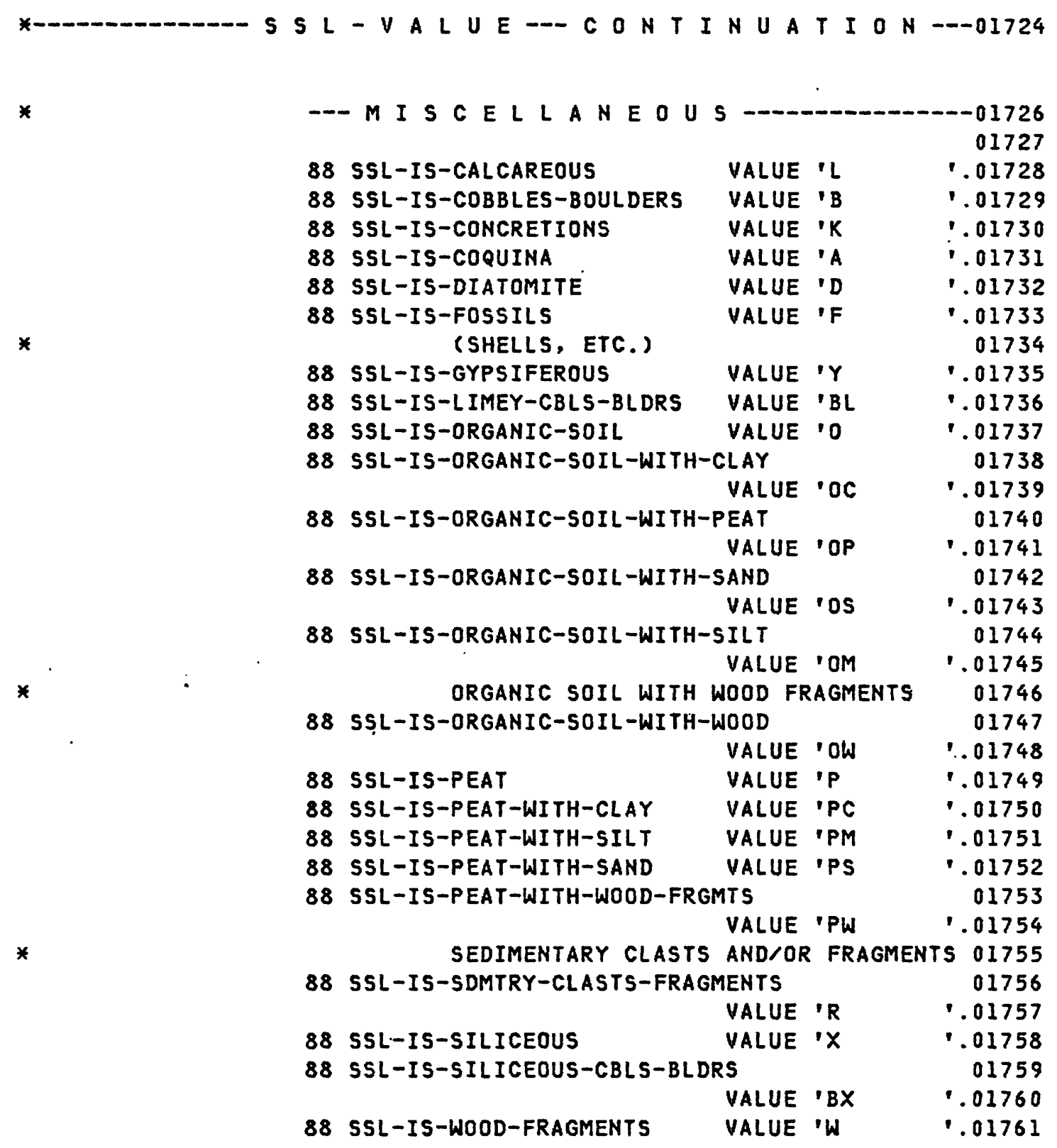

*-- S S L - VALUE-- I S C ONTINUED---01763

*--- SPEC-SMPL-LITHOLOGY-DATA-MENU --- I S C ONTINUE D---01765 
*--- SPEC-SMPL-LITHOLOGY-DATA-MENU --- C ONT I N U A T I O N---01809 SSL- VALUE-- C ONT INUATION---01811

*

*

- MAJOR COMPONENT GR O U P S -01813 01814

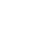

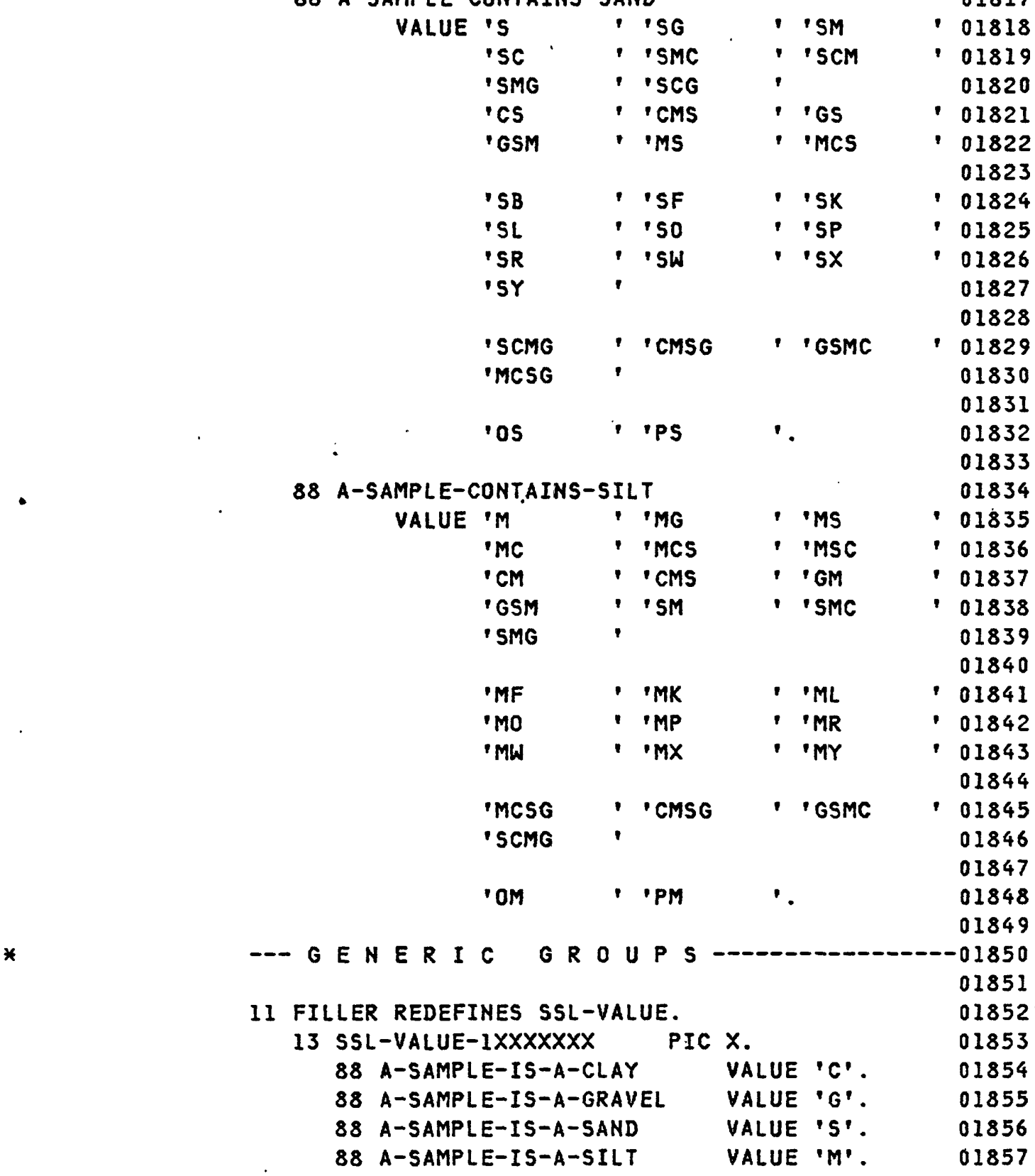

*-- SPEC-SMPL-LITHOLOGY-DATA-MENU --- I S C ONT INU E D---01859 


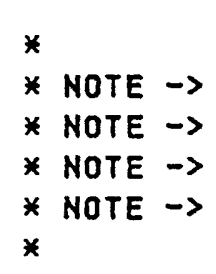

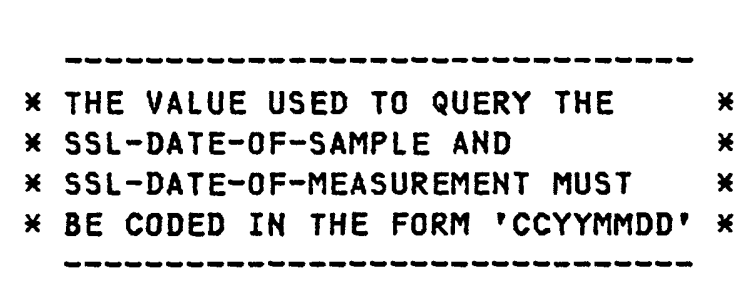

11 SSL-DATE-OF-SAMPLE.

13 SSL-DOS-CENTURY-AND-YEAR.

15 SSL-DOS-CENTURY

15 SSL-DOS-YEAR

13 SSL-DOS-MONTH

13 SSL-DOS-DAY

PIC XX.

PIC $X X$.

PIC $X X$.

PIC $X X$.

11 SSL-DATE-OF-MEASUREMENT.

13 SSL-DOM-CENTURY-AND-YEAR.
15 SSL-DOM-CENTURY

15 SSL-DOM-YEAR

13 SSL-DOM-MONTH

13 SSL-DOM-DAY

11 SSL-METHOD

88 SSL-DIRECT-OBSERVATION

PIC $X X$.

PIC $X X$.

PIC $X X$.

PIC $X X$.

PIC XX.

VALUE 'DO'.

11 SSL-SECONDARY-DATA-SOURCE.

13 SSL-SECONDARY-DATA-SOURCE-ID.

15 SSL-SECONDARY-SOURCE-CODE

PIC XXX.

15 SSL-SECONDARY-SOURCE-QUALFR

PIC XXX.

13 SSL-SECONDARY-ACTIVITY-CODE

PIC $X$.

11 SSL-DMG-INTERPRETED-CODE PIC $X$.
01863

01864

01865

01866

01867

01868

01869

01870

01871

01872

01873

01874

01875

01876

01877

01878

01879

01880

01881

01882

01883

01884

01885

01886

01887

01888

01889

01890

01891

01892

01893

01894

01895

01896

01897

*- - E N D

O F

SPEC-SMPL-LITHOLOGY-DATA-MENU

01899 
09 IND-STND-PENETRATION-TST-AVAIL

01902

01903

01904

01905

88 STND-PENETRATION-TEST-AVAIL VALUE 'Y'.

01907

09 STND-PENETRATION-TST-DATA-MENU.

01908

11 IND-SPT-VALUE-AVAIL

PIC $x$.

01909

88 SPT-VALUE-AVAIL

11 SPT-VALUE

PIC 99.

01910

01911

01912

×

* NOTE $\rightarrow$

* NOTE $\rightarrow$

* NOTE $\rightarrow$

* NOTE $\rightarrow$

*
* the value used to query the *

* SPT-DATE-OF-SAMPLE AND *

* SPT-DATE-OF-MEASUREMENT MUST *

* BE CODED IN THE FORM 'CCYYMMDD' *
01913

01914

01915

01916

01917

01918

01919

01920

01921

01922

01923

01924

01925

01926

01927

01928

01929

01930

01931

01932

01933

01934

01935

01936

01937

01938

01939

01940

01941

01942

01943

01944

01945

01946 
09 IND-TAN-PHI-AVAIL 88 TAN-PHI-AVAIL

PIC $x$. VALUE 'Y'.

01951

01952

01953

01954

09 TAN-PHI-DATA-MENU.

11 IND-TAN-VALUE-AVAIL 88 TAN-VALUE-AVAIL

11 TAN-VALUE
PIC $X$. VALUE ' $Y$ '. PIC 9V999.
01955

01956

01957

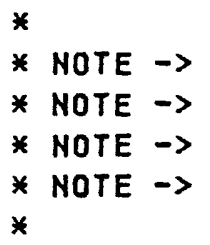

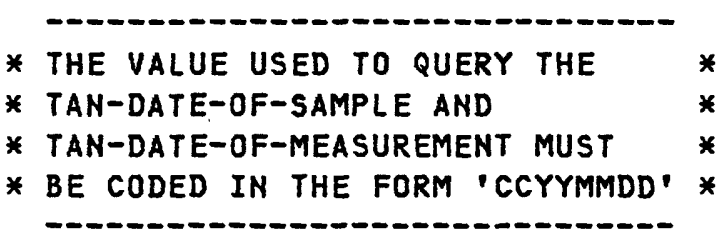

II TAN-DATE-OF-SAMPLE.

13 TAN-DOS-CENTURY-AND-YEAR. $\begin{array}{ll}15 \text { TAN-DOS-CENTURY } & \text { PIC XX. } \\ 15 \text { TAN-DOS-YEAR } & \text { PIC XX. } \\ \text { TAN-DOS-MONTH } & \text { PIC XX. } \\ \text { TAN-DOS-DAY } & \text { PIC XX. }\end{array}$

11 TAN-DATE-OF-MEASUREMENT.

13 TAN-DOM-CENTURY-AND-YEAR. 15 TAN-DOM-CENTURY 15 TAN-DOM-YEAR

13 TAN-DOM-MONTH

13 TAN-DOM-DAY

11 TAN-METHOD 88 TAN-CALCULATED
PIC XX. PIC $X X$. PIC $X X$. PIC $X X$.

PIC $X X$. value ' $c A$ '.

01958

01959

01960

01961

01962

01963

01964

01965

01966

01967

01968

01969

01970

01971

01972

01973

01974

01975

01976

01977

01978

01979

01980

01981

01982

01983

01984

01985

01986

01987

01988

13 TAN-SECONDARY-ACTIVITY-CODE

PIC XXX.

11 TAN-DMG-INTERPRETED-CODE PIC $X$. 
09 IND-TRUE-SPEC-GRAVITY-AVAIL PIC $X$.

09 TRUE-SPEC-GRAVITY-DATA-MENU.

11 IND-TSG-VALUE-AVAIL 88 TSG-VALUE-AVAIL

PIC $X$.

02000

11 TSG-VALUE

yalue 'Y'.

02001

02002

PIC 99V99.

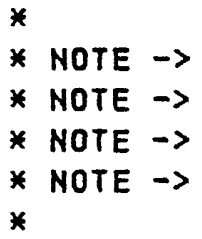

* the value used to query the

02004

02005

02006

* TSG-DATE-OF-SAMPLE AND *

02007

* TSG-DATE-OF-MEASUREMENT MUST *

* BE CODED IN THE FORM 'CCYYMMDD' *

02008

02009

02010

02011

11 TSG-DATE-OF-SAMPLE.

02012

13 TSG-DOS-CENTURY-AND-YEAR.

$\begin{array}{ll}15 \text { TSG-DOS-CENTURY } & \text { PIC XX. } \\ 15 \text { TSG-DOS-YEAR } & \text { PIC XX. } \\ \text { TSG-DOS-MONTH } & \text { PIC XX. } \\ \text { TSG-DOS-DAY } & \text { PIC XX. }\end{array}$

02013

02014

02015

02016

02017

02018

11 TSG-DATE-OF-MEASUREMENT.

02019

13 TSG-DOM-CENTURY-AND-YEAR .

$$
\begin{aligned}
& 15 \text { TSG-DOM-CENTURY } \\
& 15 \text { TSG-DOM-YEAR }
\end{aligned}
$$

13 TSG-DOM-MONTH

13 TSG-DOM-DAY

11 TSG-METHOD

88 TSG-RATIO
PIC $X X$.

PIC $X X$.

PIC $X X$.

PIC $X X$.

PIC $X X$. VALUE 'RO'.

11 TSG-SECONDARY-DATA-SOURCE.

13 TSG-SECONDARY-DATA-SOURCE-ID.

15 TSG-SECONDARY-SOURCE-CODE

02030

02031

02032

15 TSG-SECONDARY-SOURCE-QUALFR

02033

02034

13 TSG-SECONDARY-ACTIVITY-CODE PIC XXX.

02035

02036

02037

11 TSG-DMG-INTERPRETED-CODE PIC $X$. 
07 UNIT-COHESION-MENU.

02040

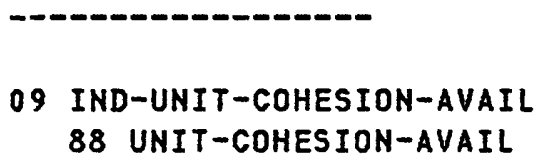

11 UC-DATE-OF-SAMPLE.

13 UC-DOS-CENTURY-AND-YEAR.

$\begin{array}{ll}15 \text { UC-DOS-CENTURY } & \text { PIC } X X . \\ 15 \text { UC-DOS-YEAR } & \text { PIC XX. } \\ \text { UC-DOS-MONTH } & \text { PIC XX. } \\ \text { UC-DOS-DAY } & \text { PIC XX. }\end{array}$

02059

02060

02061

02062

02063

02064

02065

02066

02067

02068

02069

13 UC-DOM-MONTH

PIC $X X$.

PIC $X X$.

PIC $X X$.

02070

13 UC-DOM-DAY

PIC $X X$.

02071

02072

11 UC-METHOD

PIC $X X$.

88 UC-INSTRUMENT-MEASUREMENT VALUE 'IM'. 02073

11 UC-SECONDARY-DATA-SOURCE.

02075

13 UC-SECONDARY-DATA-SOURCE-ID.

02076

15 UC-SECONDARY-SOURCE-CODE PIC XXX.

02077

02078

15 UC-SECONDARY-SOURCE-QUALFR

02079 PIC XXX.

13 UC-SECONDARY-ACTIVITY-CODE

11 UC-DMG-INTERPRETED-CODE PIC $X$. 
09 IND-VOID-RATIO-AVAIL 88 VOID-RATIO-AVAIL

PIC $X$.

02089

value 'Y'.

02090

09 VOID-RATIO-DATA-MENU.

11 IND-VR-VALUE-AVAIL 88 VR-VALUE-AVAIL

PIC $x$.

02091

02092

11 VR-VALUE

VALUE ' $Y$ '.

02093

02094

PIC 9V99.

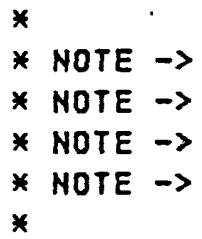

* the value used to query the

02096

02097

02098

* UR-DATE-OF-SAMPLE AND *

02099

* UR-DATE-OF-MEASUREMENT MUST *

02100

* BE CODED IN THE FORM 'CCYYMMDD' * 02101

02102

02103

11 VR-DATE-OF-SAMPLE.

02104

13 UR-DOS-CENTURY-AND-YEAR.

15 VR-DOS-CENTURY PIC XX.

15 UR-DOS-YEAR PIC $X X$.

02105

02106

13 VR-DOS-MONTH

PIC $X X$.

02107

13 UR-DOS-DAY

PIC $X X$.

02108

02109

02110

11 VR-DATE-OF-MEASUREMENT.

02111

13 UR-DOM-CENTURY-AND-YEAR .

15 VR-DOM-CENTURY

15 VR-DOM-YEÄR

13 VR-DOM-MONTH

13 VR-DOM-DAY

PIC $X X$.

PIC $X X$.

02112

02113

02114

02115

02116

02117

11 VR-METHOD

88 VR-RATIO

PIC XX.

02118

02119

02120

02121

02122

02123

02124

02125

02126

02127

13 VR-SECONDARY-ACTIVITY-CODE PIC XXX.

02128

02129

11 VR-DMG-INTERPRETED-CODE PIC $X$. 
*

*

* NOTE $\rightarrow$

* NOTE $\rightarrow$

*

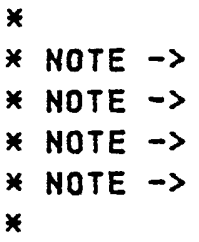

07 WATER-TABLE-MENU. $\quad 02132$

02133

02134

09 IND-WATER-TABLE-AVAIL

PIC $X$.

02135

88 WATER-TABLE-AVAIL

VALUE 'Y'.

02136

02137

02138

02139

02140

02141

02142

02143

02144

11 IND-WT-VALUE-AVAIL

PIC $X$.

02145 88 WT-VALUE-AVAIL

VALUE ' $Y$ '. 02146

11 WT-VALUE

PIC 9(5)V9.

02147

02148

02149

02150

02151

02152

02153

02154

02155

02156

02157

02158

02159

02160

02161

02162

02163

02164

02165

02166

02167

02168

02169

02170

02171

02172

02173

02174

02175

02176

02177

02178

02179

02180

02181

02182

02183

02184

II WT-DMG-INTERPRETED-CODE PIC $x$. 
09 IND-YOUNGS-MODULUS-AVAIL 88 YOUNGS-MODULUS-AVAIL

O9 YOUNGS-MODULUS-DATA-MENU.

11 IND-YM-VALUE-AVAIL 88 YM-VALUE-AVAIL

11 YM-VALUE
PIC $x$.

VAlUe ' $Y$ '.

02187

02188

02189

02190

02191

02192

02193

02194

02195

02196

02197

02198

02199

02200

02201

02202

02203

02204

02205

02206

02207

02208

02209

02210

02211

02212

02213

02214

02215

02216

02217

02218

02219

02220

02221

02222

02223

02224

02225

02226

02227

02228

02229

02230

02231

02232

02233

11 YM-DMG-INTERPRETED-CODE PIC $X$. 


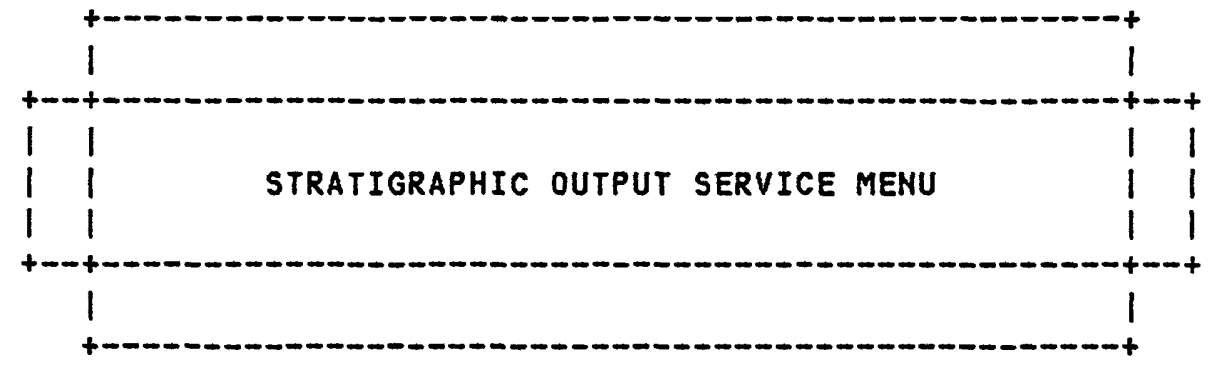


OI STRAT-OUTPUT-SERVICE-MENUS. STRAT-OUTPUT-SERVICE-MENUS

5 CURRENT AS OF 25 JUN 1980 (DEF REF: GDQ.AAMP)

$09 / 16 / 80$ DICTGDQ I F OR S T RA T I GR A P H I C D A TA THE FOLLOWING "DATA NAMES" ARE THOSE WHICH MAY BE USED TO SPECIFY WHICH DATA ITEMS AND WHICH STRATIGRAPHIC PARAMETERS ARE TO BE PRINTED.

CODING INSTRUCTIONS

00019

00020

00021

TO SPECIFY THAT A PARTICULAR DATA ITEM IS TO

BE PRINTED CODE "MOVE YES TO DATA-NAME".

EXAMPLE: MOVE YES TO PRINT-RELATIVE-DENSITY PRINT-PARAMETER-VALUE

EXAMPLE: MOVE YES TO PRINT-DEPTH-TO-TOP PRINT-THICKNESS PRINT-RELATIVE-GEOLOGIC-AGE PRINT-DEPTH-TO-SAMPLE PRINT-APRNT-SPEC-GRAVITY PRINT-UNIT-COHESION

NOTE: THE CALIFORNIA COORDINATE VALUE IS ALWAYS PRINTED IF ANY OTHER DATA NAME IS REQUESTED.

IF ONLY A LIST OF CALIFORNIA COORDINATE YALUES WHICH SATISFY 00022 00023 00024 00025 00026 00027 00028 00029 00030 00031 00032 00033 00034 00035 00036 00037 00038 00039 00040 THE QUERY IS DESIRED THEN CODE 00041 00042 "MOVE YES TO PRINT-CALIF-COORDINATES" 00043 00044 AS THE ONLY PRINT REQUEST. 
* +

*--I PRINT SER VI CE MENU 1--

* +

00053

00054

05 PRINT-ALL-DATA-ITEMS

PIC XXX. $\quad 00056$

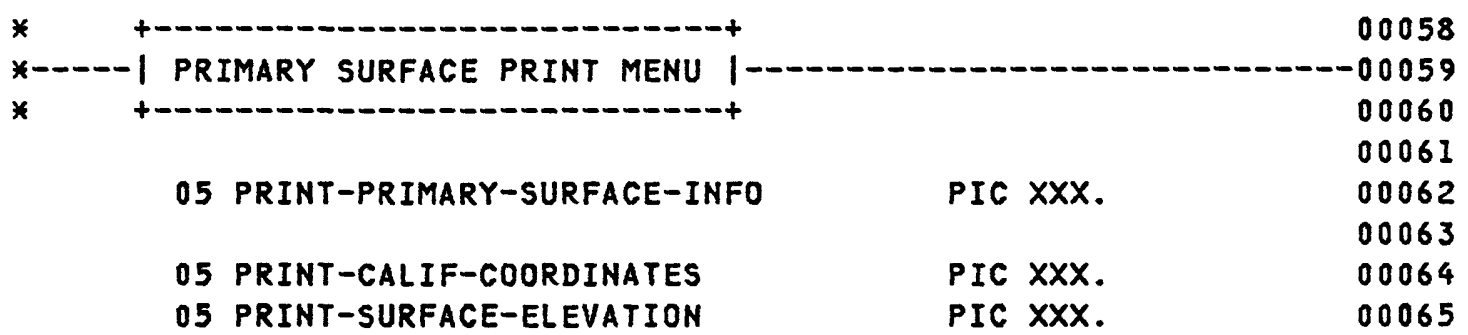

* + +

*--- -1 SECONDARY SURFACE PRINT MENU 1-

* + +

05 PRINT-SECONDARY-SURFACE-INFO PIC XXX.

O5 PRINT-LONGITUDE $\quad$ PIC XXX. 00073

05 PRINT-LATITUDE $\quad$ PIC XXX. 00074

05 PRINT-STATE PIC XXX. . 00075

05 PRINT-COUNTY PIC XXX.

05 PRINT-ATLAS-SHEET PIC XXX. 00077

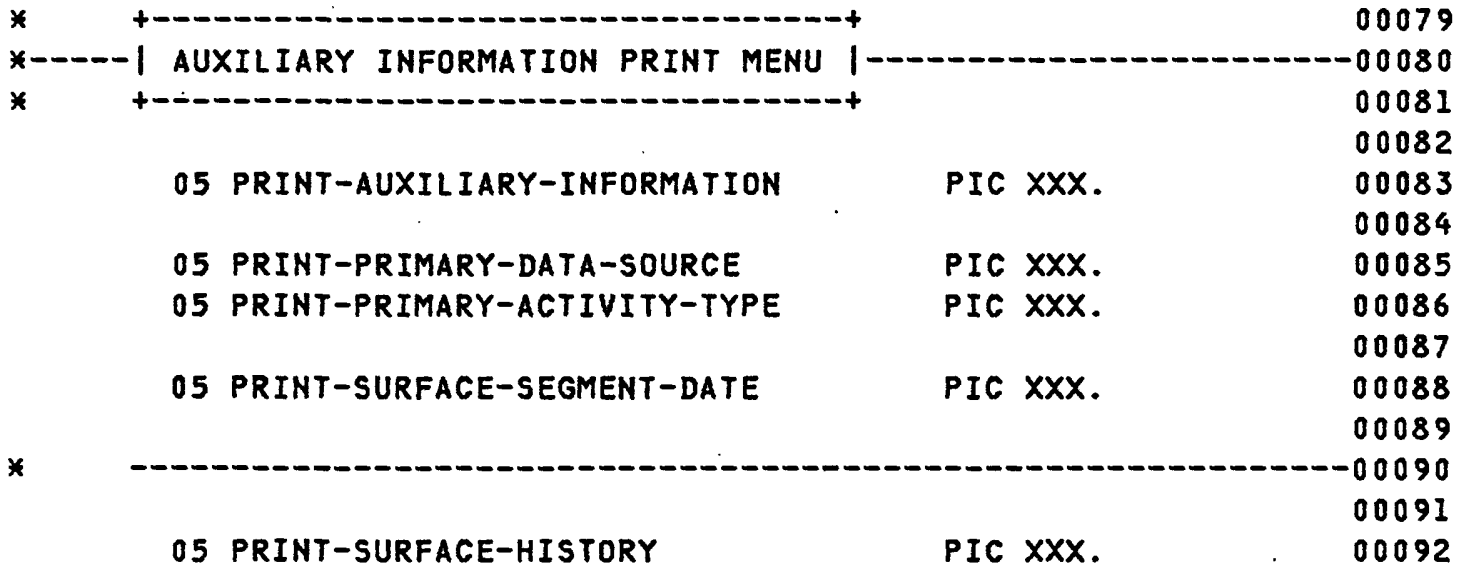


*--- 1 LITHOLOGIC LAYER PRINT MENU

*
+ LITHOLOGIC LAYER PRINT MENU 1

05 PRINT-LITHOLOGIC-LAYER-INFO

05 PRINT-DEPTH-TO-LAYER-TOP

05 PRINT-THICKNESS-OF-LAYER

05 PRINT-GENERAL-LITHOLOGY

05 PRINT-STRATIGRAPHIC-ID

05 PRINT-STRATIGRAPHIC-GROUP

05 PRINT-STRATIGRAPHIC-FORMATION

05 PRINT-STRATIGRAPHIC-MEMBER

05 PRINT-RELATIVE-GEOL-AGE

05 PRINT-RELATIVE-GEOL-ERA

05 PRINT-RELATIVE-GEOL-PERIOD

05 PRINT-RELATIVE-GEOL-SER-EPH

05 PRINT-RELATIVE-GEOL-STAGE

05 PRINT-DETERMINATION-METHOD

05 PRINT-LAYER-SEGMENT-DATE $+\quad 00094$

-

00096

00097

00098

00099

00100

00101

00102

00103

00104

00105

00106

00107

00108

00109

00110

00111

00112

00113

00114

00115

00116

00117

00118

00119

$-00120$

00121

05 PRINT-LITHOLOGIC-LAYER-HISTORY PIC XXX.

00122 


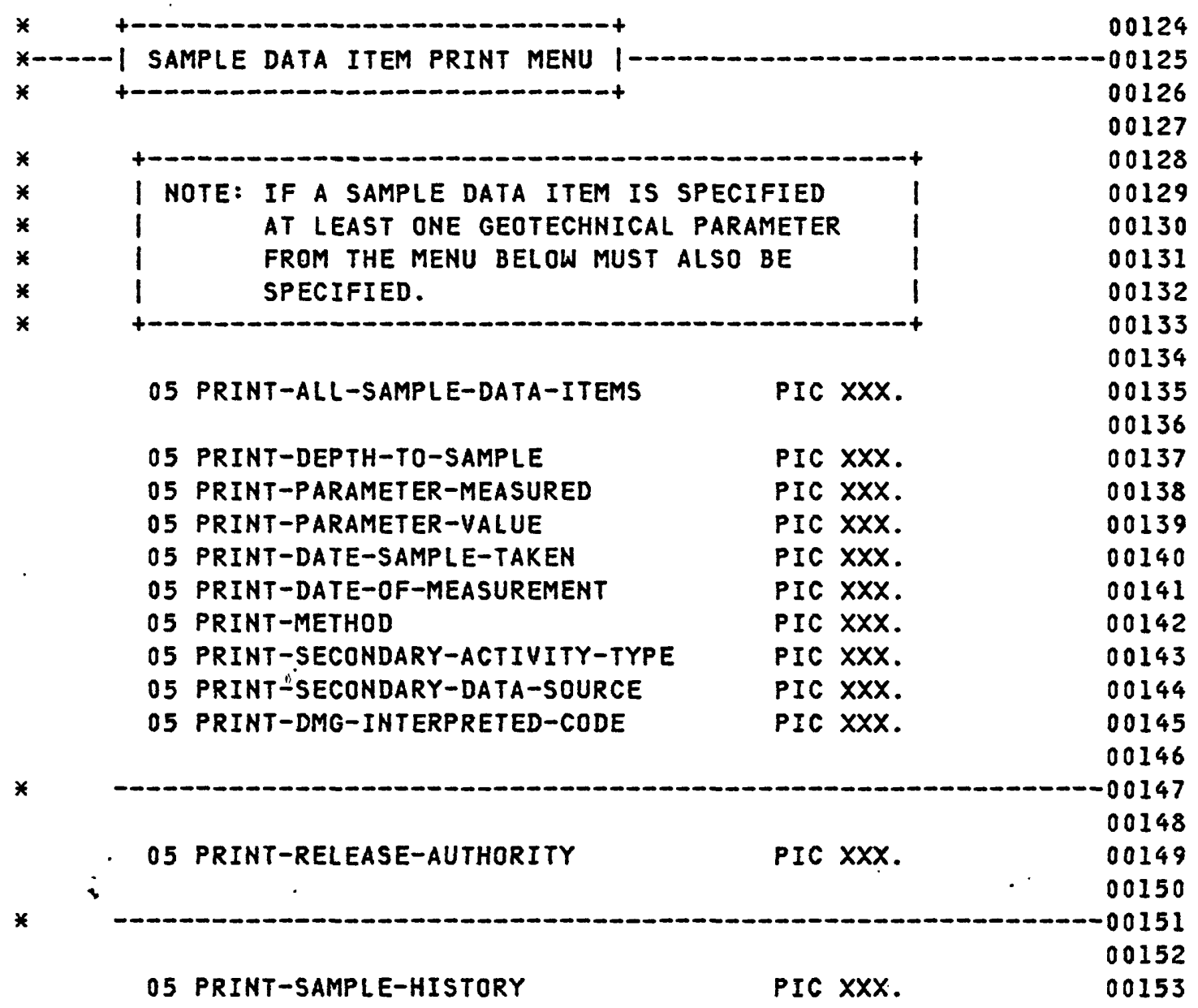




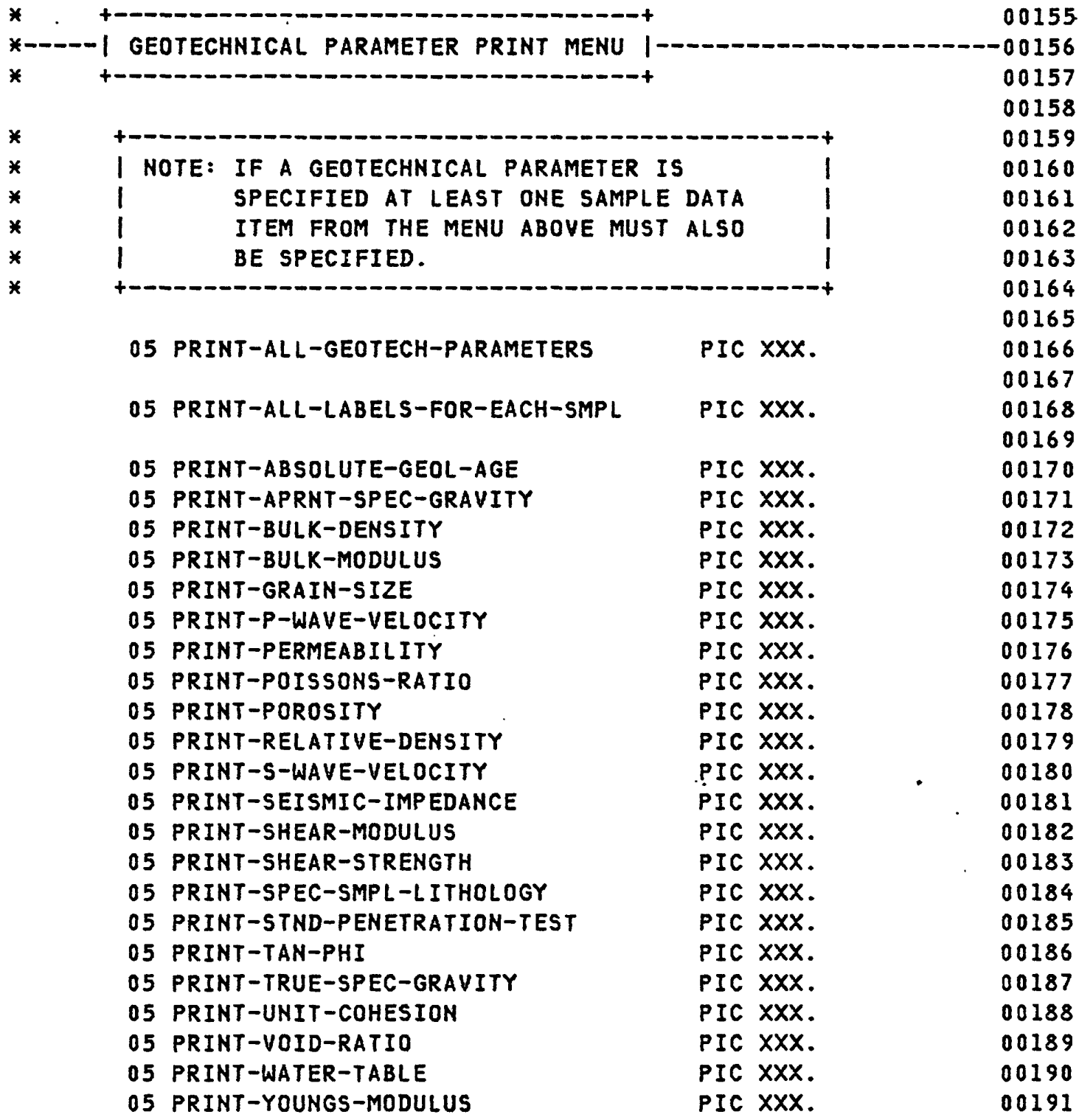

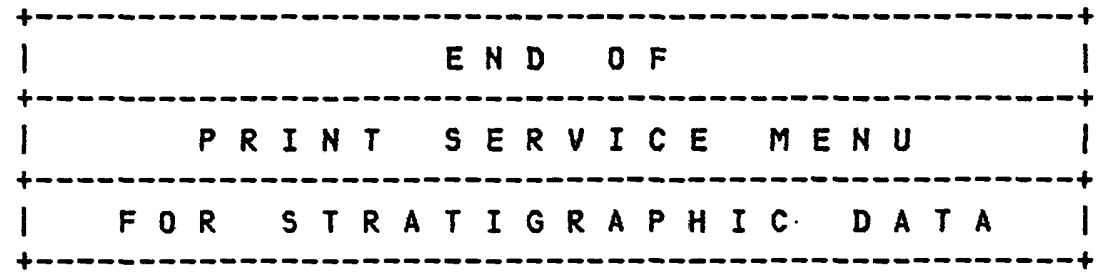




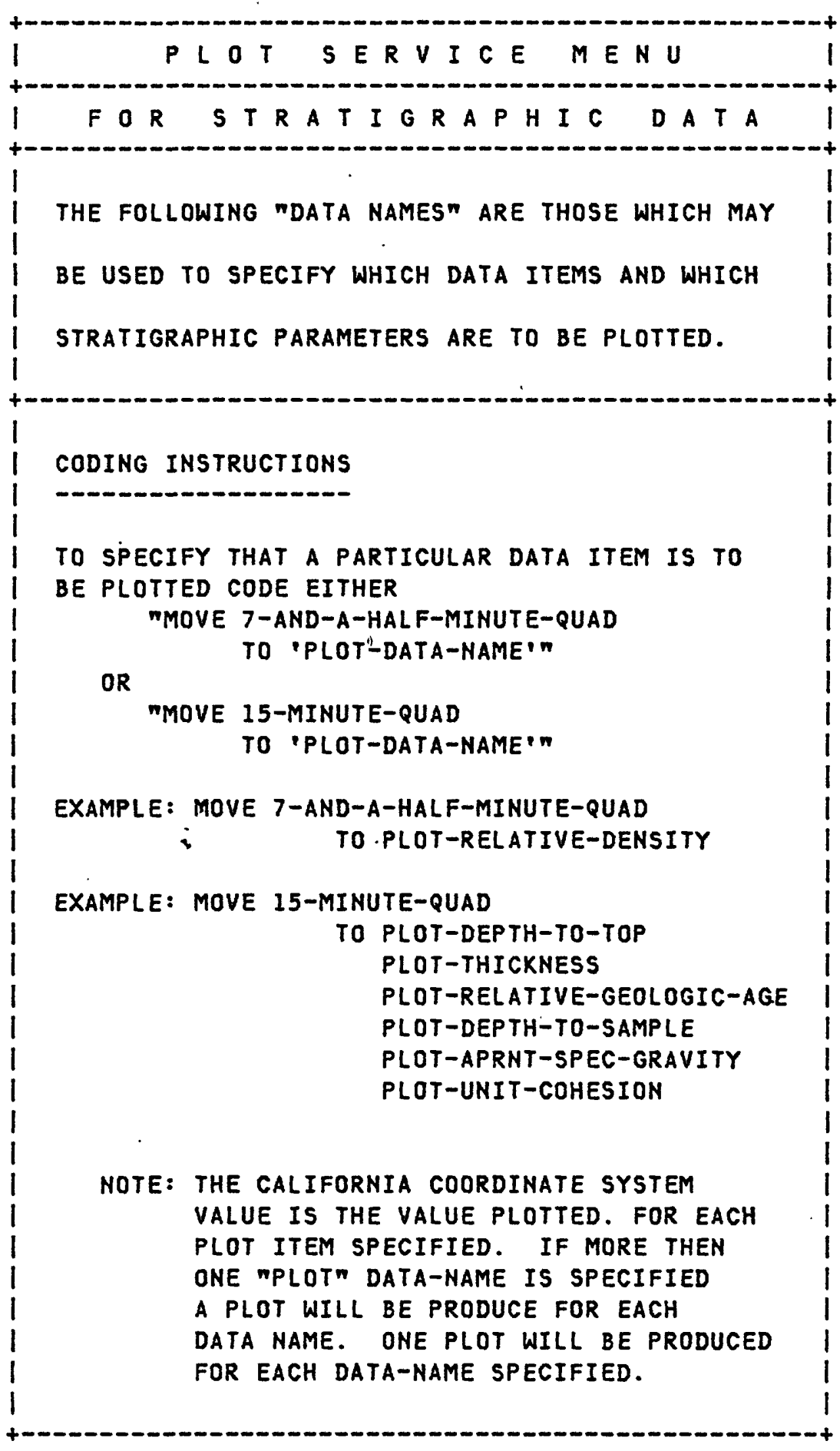




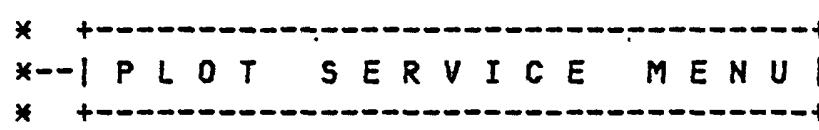

00248

*--1 P L O T SER V I C E MEN U 1-

* +

00250

00251

O3 PLOT-SERVICE-MENU. $\quad 00252$

00253

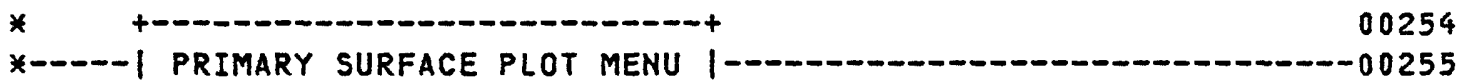

* + +-

00257

05 PLOT-CALIF-COORDINATES

PIC $X X X$.

00258

05 PLOT-SURFACE-ELEVATION

PIC $X X X$.

00259
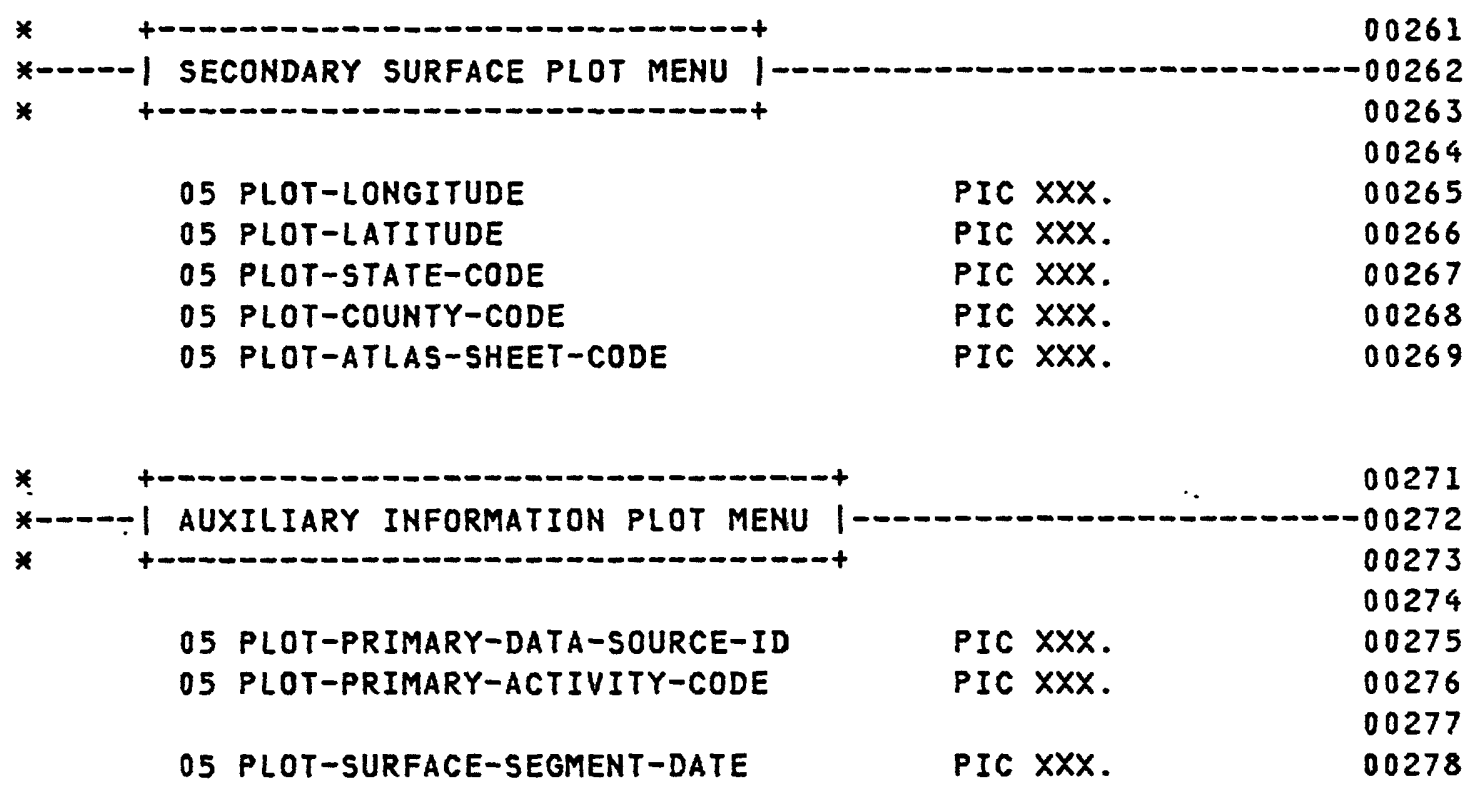


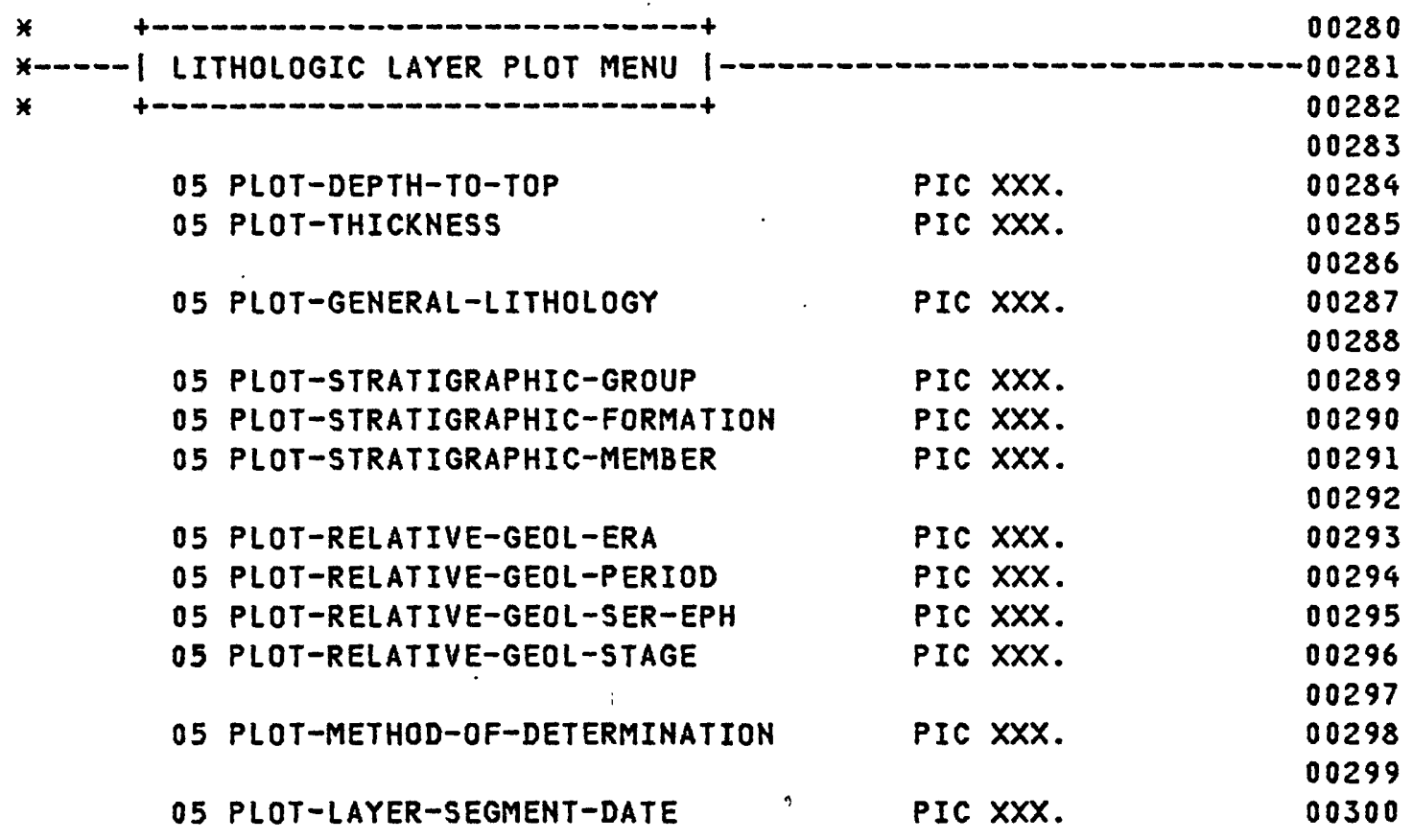

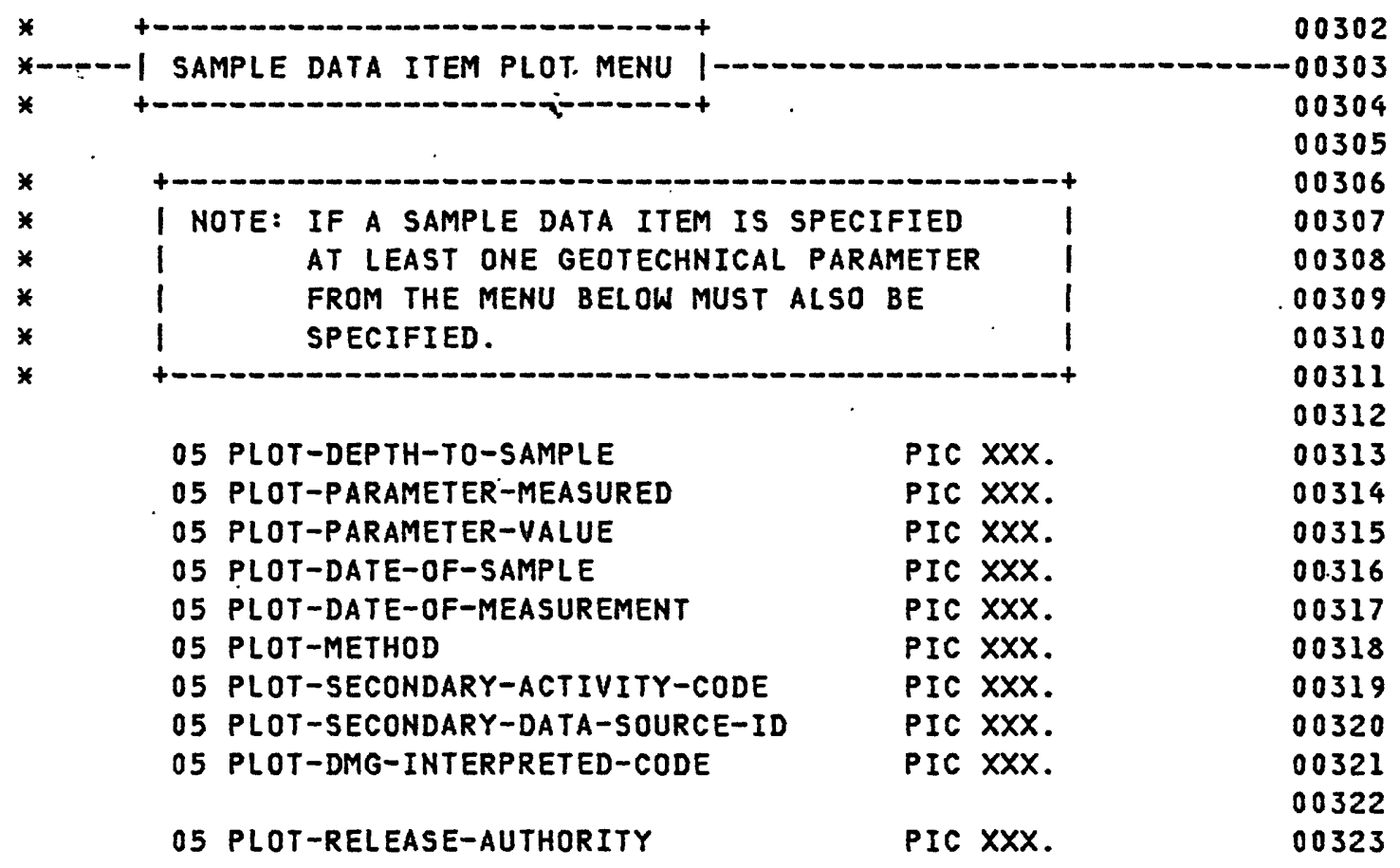




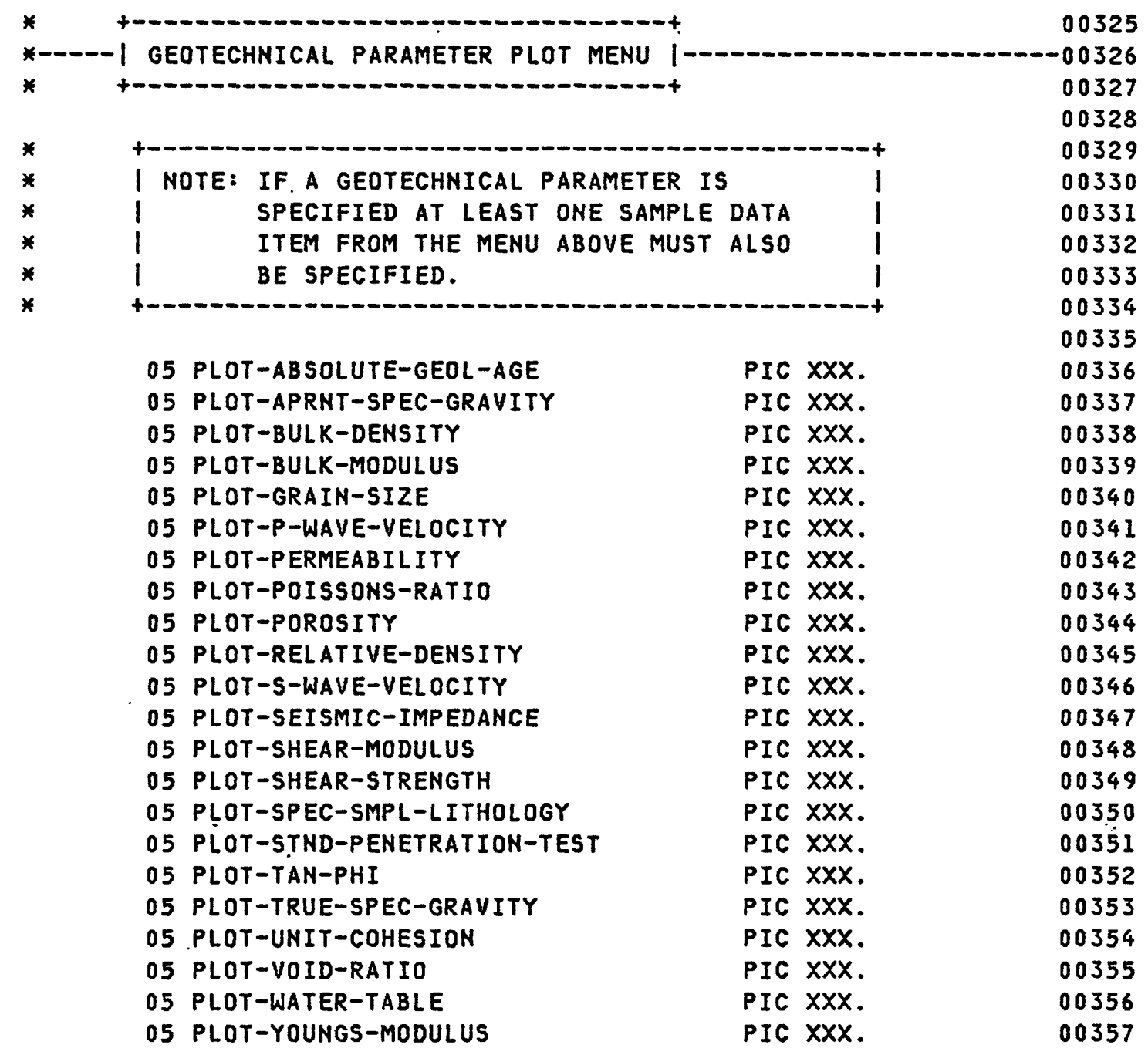

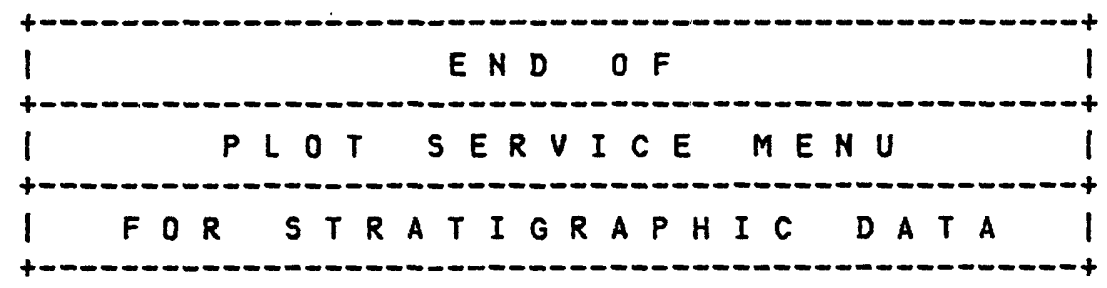

00359

00360

00361

00362

00363

00364

00365 


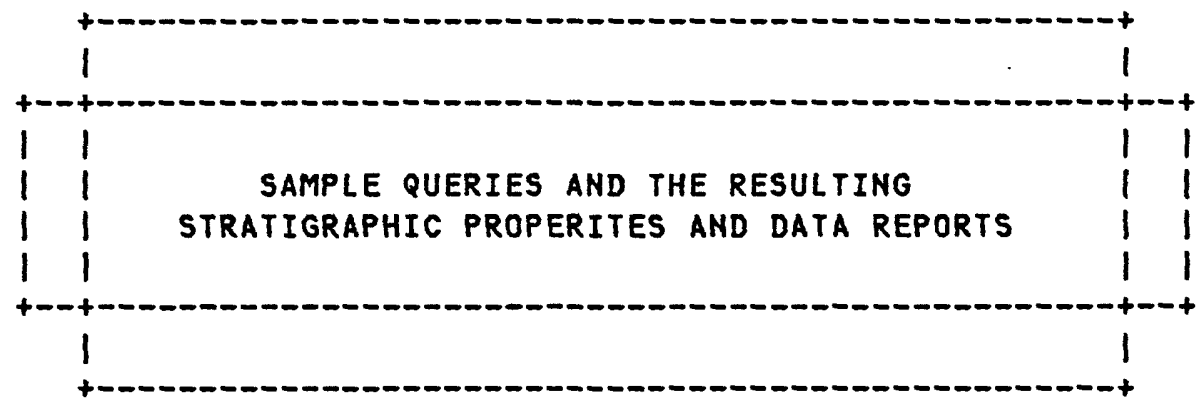

THE SAMPLE QUERIES AND RESULTING STRATIGRAPHIC PROPERTIES AND DATA REPORTS PROVIDED AS THIS ATTACHMENT WERE PROCESSED AGAINST A STORAGE FILE GENERATED BY THE SAMPLE STRATIGRAPHIC EDIT/AUDIT AND CHANGE REPORTS PROVIDED PROVIDED AS ATTACHMENT B. 


\begin{tabular}{|c|c|c|}
\hline $\begin{array}{l}\text { QUERY } \\
\text { NAME }\end{array}$ & DESCRIPTION & PAGE \\
\hline PRTAVAIL & $\begin{array}{l}\text { THIS REPORT SHOWS ALL DATA AVAILABLE ON } \\
\text { THE STORAGE FILE. }\end{array}$ & GI-2 \\
\hline PRINTALL & $\begin{array}{l}\text { THIS REPORT SHOWS ALL DATA AVAILABLE ON } \\
\text { THE STORAGE FILE AND INCLUDES ALL THE } \\
\text { PARAMETER LABELS WHETHER DATA FOR A } \\
\text { PARTICULAR PARAMETER WAS STORED FOR THE } \\
\text { SAMPLE OR NOT. }\end{array}$ & $G 3-7$ \\
\hline PRTSURF & $\begin{array}{l}\text { THIS REPORT SHOWS A REQUEST FOR ALL } \\
\text { SURFACE DATA FROM THE STORAGE FILE. }\end{array}$ & G8 \\
\hline PRTLAYER & $\begin{array}{l}\text { THIS REPORT SHOWS A REQUEST FOR ALL } \\
\text { LAYER DATA. FROM THE STORAGE FILE. }\end{array}$ & G9 \\
\hline PRTPARM & $\begin{array}{l}\text { THIS REPORT SHOWS A REQUEST FOR ALL } \\
\text { PARAMETER DATA FROM THE STORAGE FILE. }\end{array}$ & G10 \\
\hline PRTCCL & $\begin{array}{l}\text { THIS REPORT SHOWS A REQUEST FOR A LIST } \\
\text { OF ALL CALIFORNIA COORDINATE VALUES ON } \\
\text { THE STORAGE FILE. }\end{array}$ & G11-12 \\
\hline QUERYOOI & $\begin{array}{l}\text { THIS REPORT SHOWS A REQUEST FOR ANY } \\
\text { PARAMETER WHICH WAS MEASURED USING A } \\
\text { STANDARD METHOD OR THE GENERAL LITHOLOGY } \\
\text { IS SAND. }\end{array}$ & G13-14 \\
\hline QUERYO02 & $\begin{array}{l}\text { THIS REPORT SHOWS A REQUEST IDENTICAL } \\
\text { TO QUERYOOI WHICH INCLUDES TWO ADDITIONAL } \\
\text { DATA ITEMS. }\end{array}$ & G15-16 \\
\hline CONTROLS) & THIS IS THE USER RUN PROFILE FOR QUERYOO2. & GI7 \\
\hline CONTROLS) & $\begin{array}{l}\text { THIS IS THE RUN CONROLS REPORT FOR } \\
\text { QUERYOO2. }\end{array}$ & G18 \\
\hline NODATA & $\begin{array}{l}\text { THIS REPORT SHOWS A REQUEST FOR WHICH NO } \\
\text { DATA WAS FOUND ON THE STORAGE FILE }\end{array}$ & G19 \\
\hline
\end{tabular}




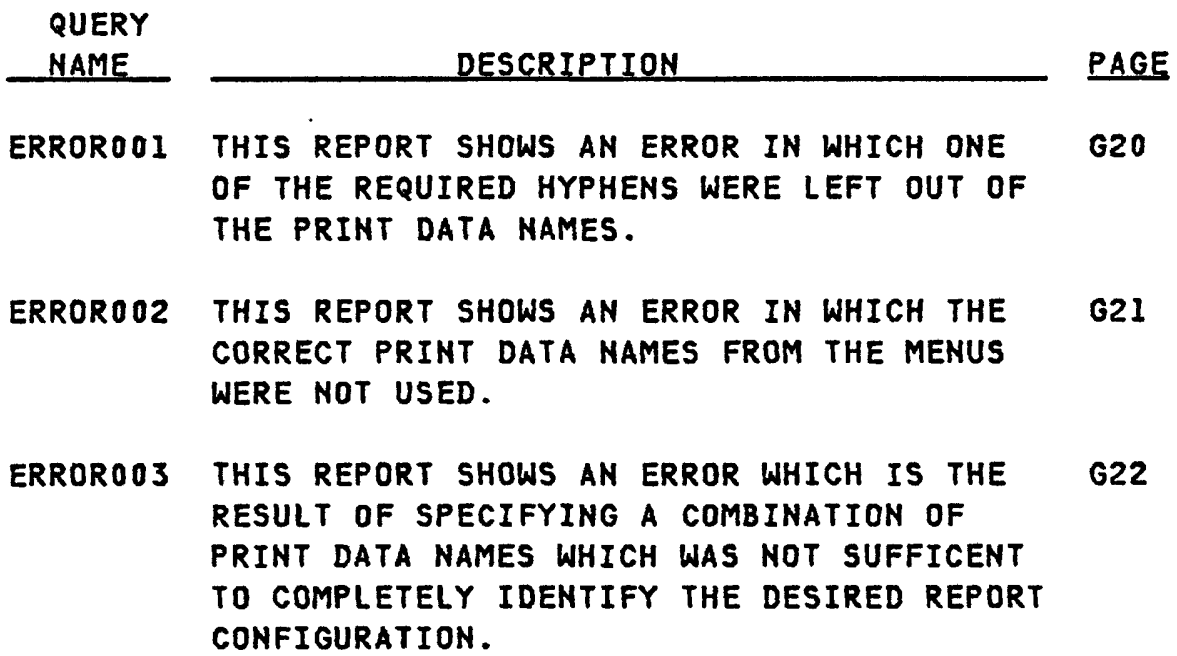




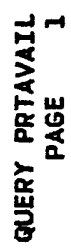

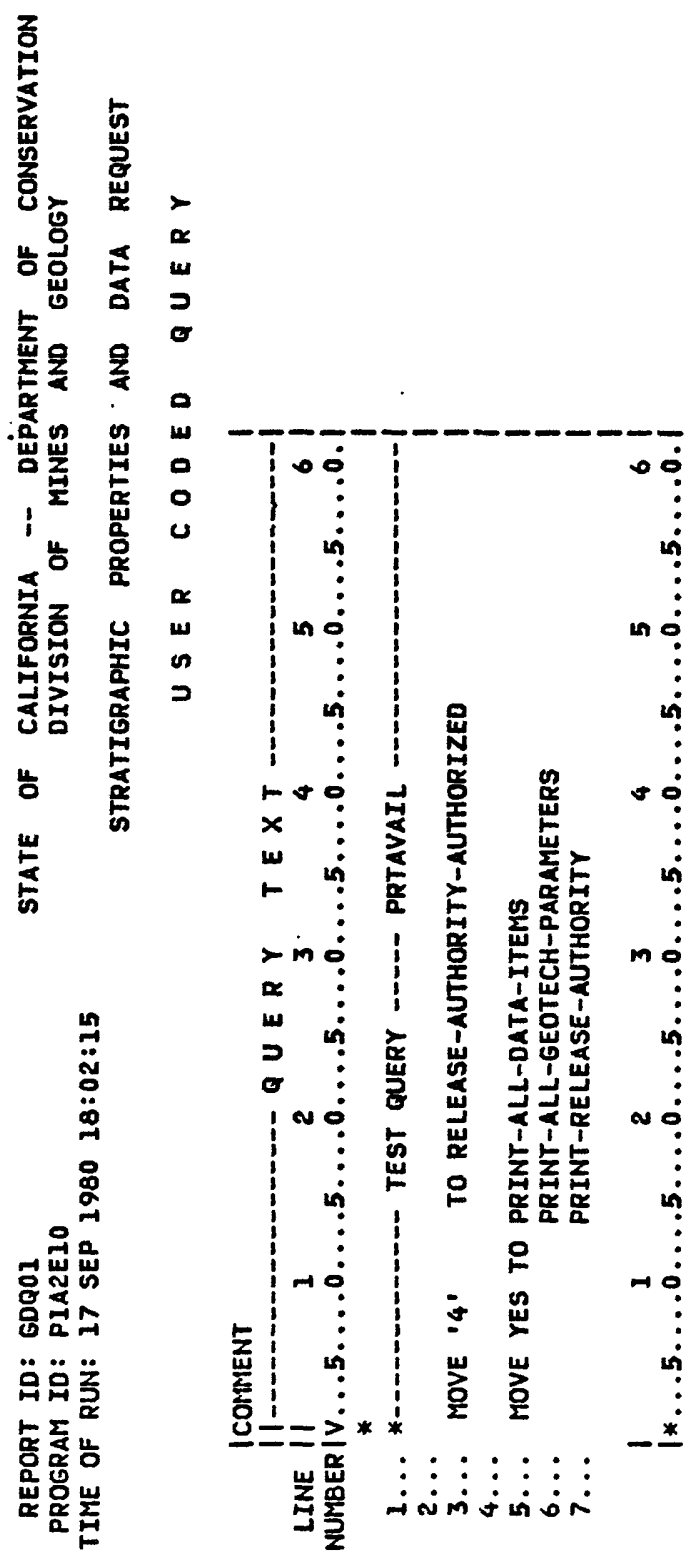




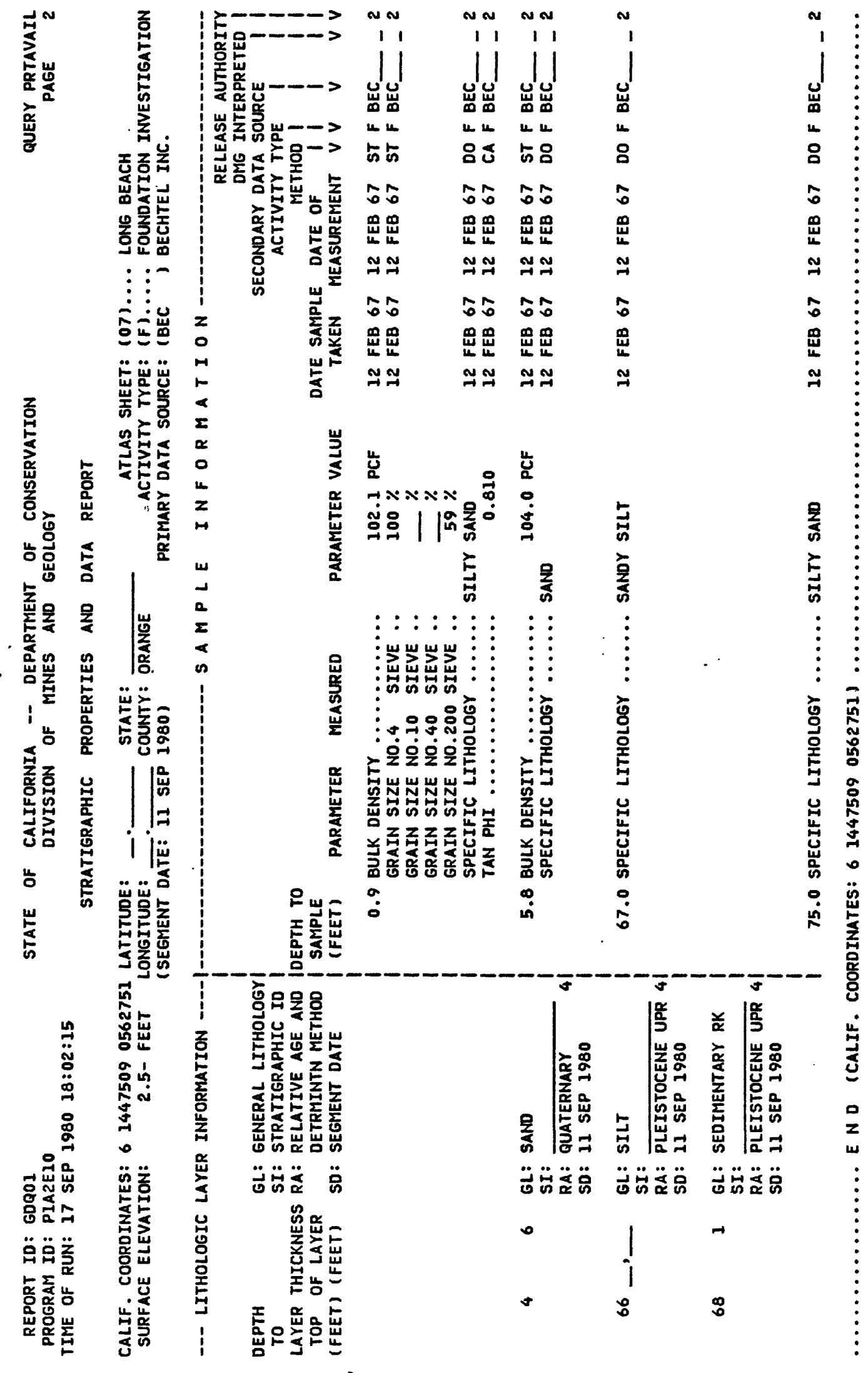




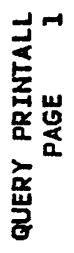

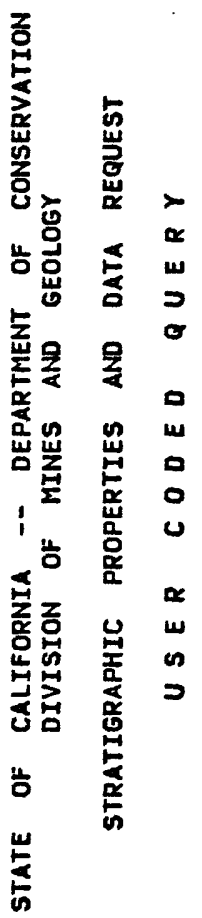

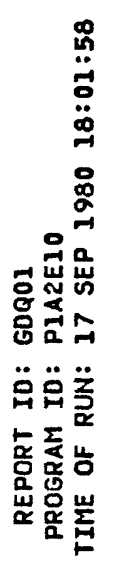

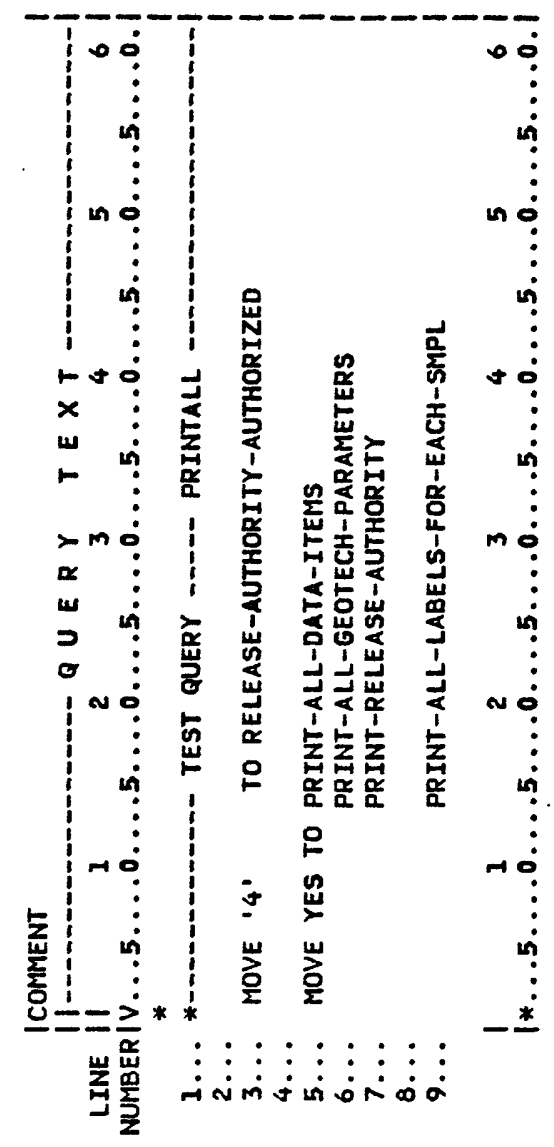




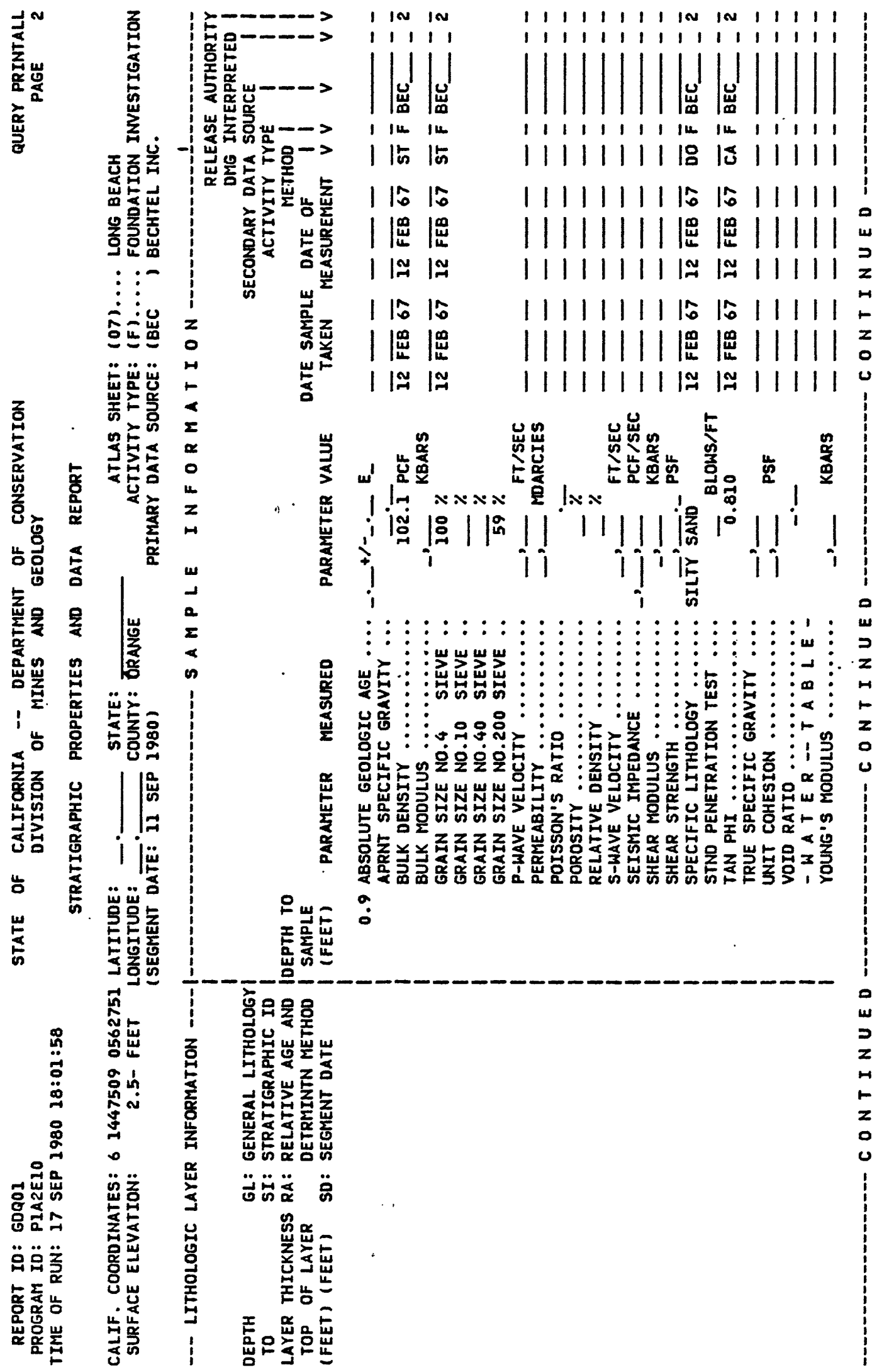




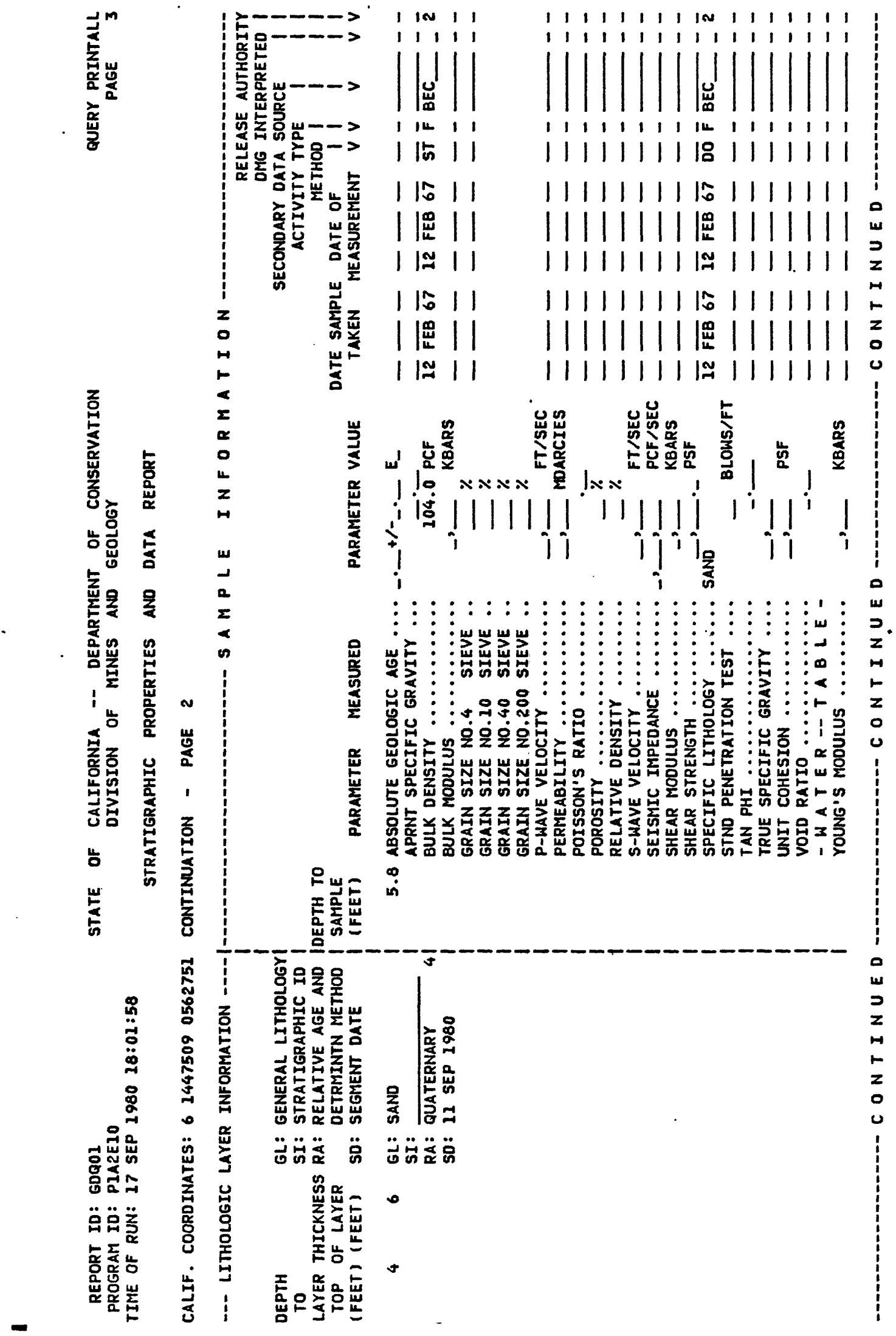




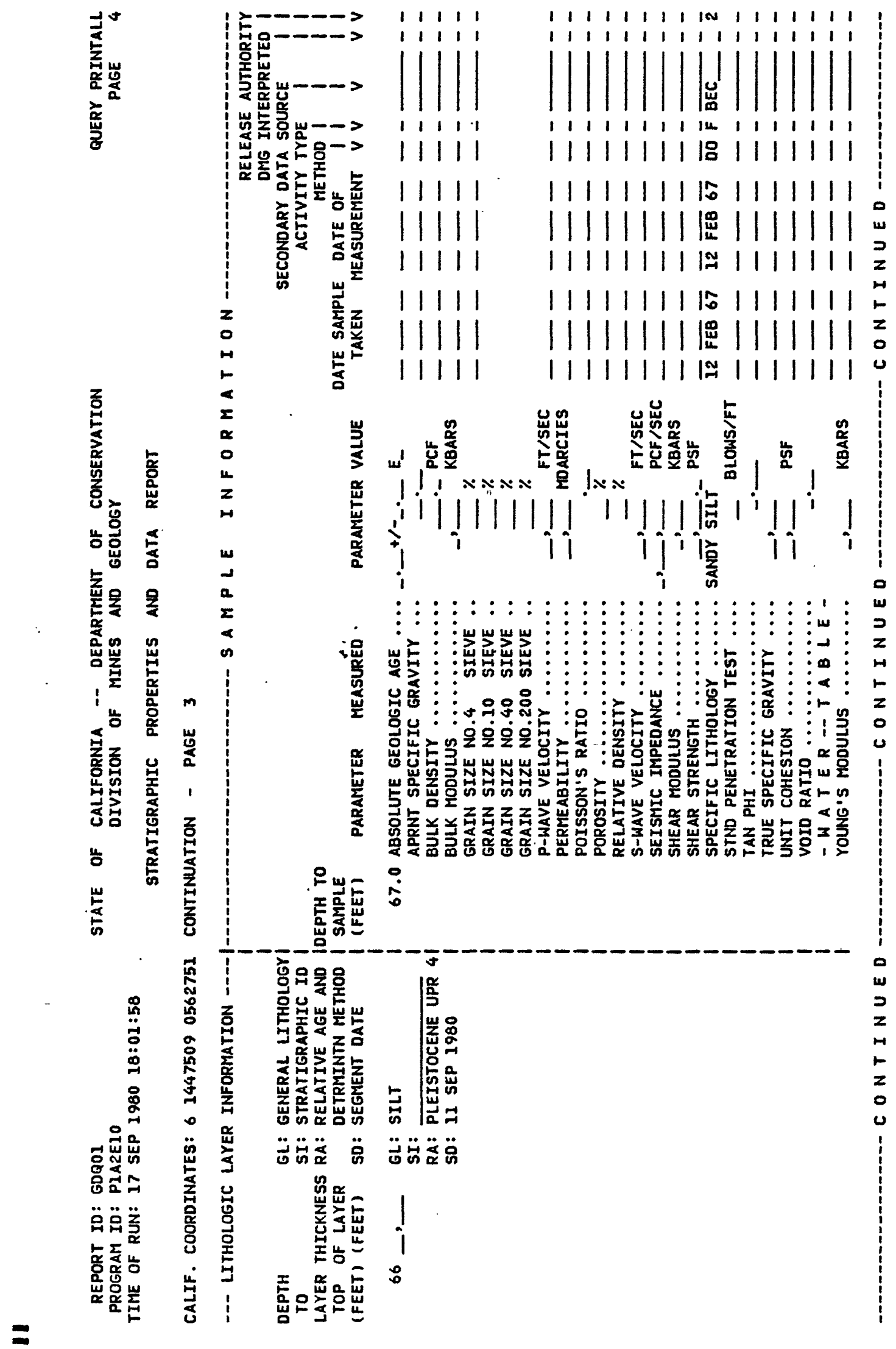



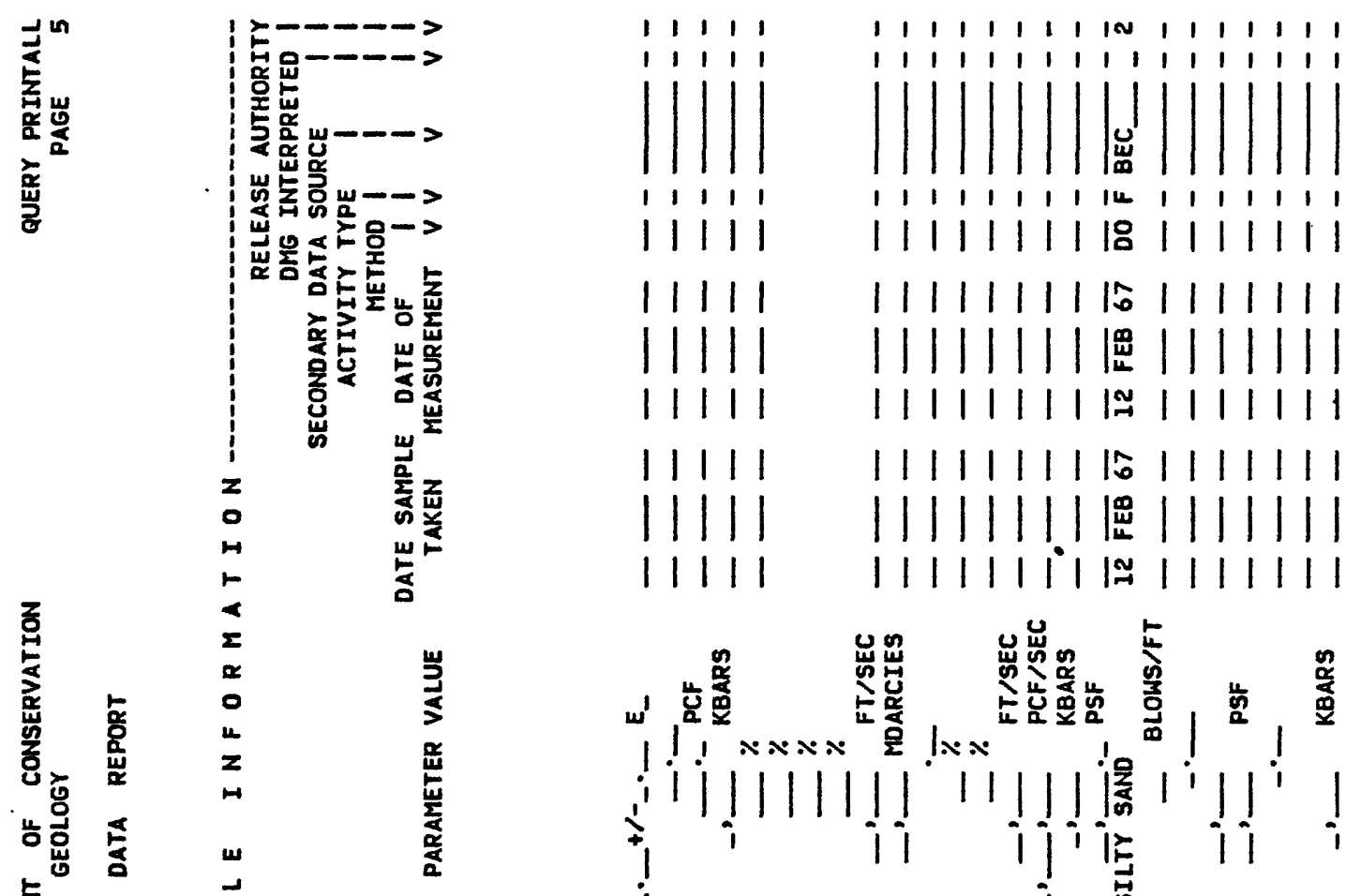

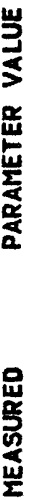
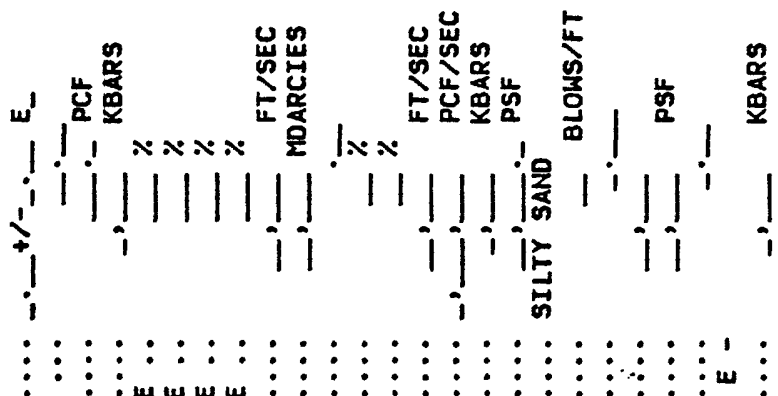

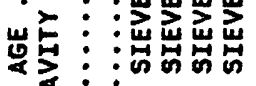

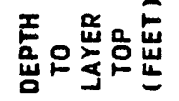



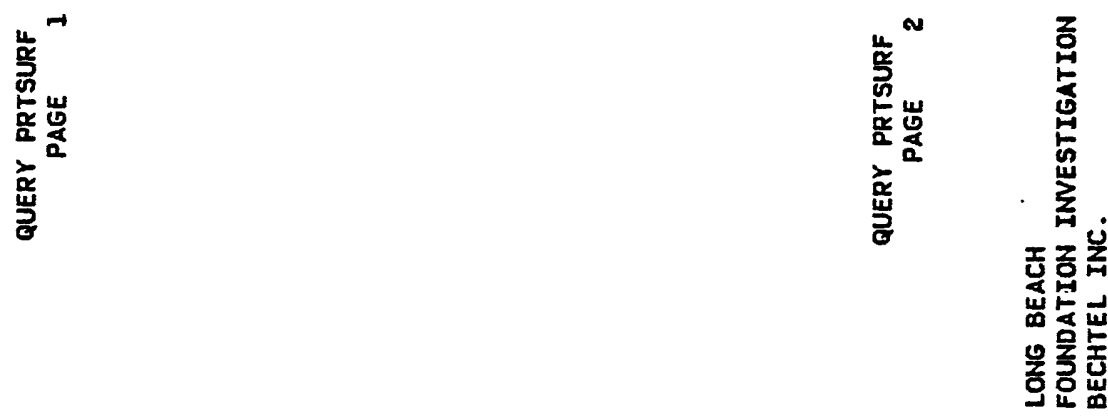

总
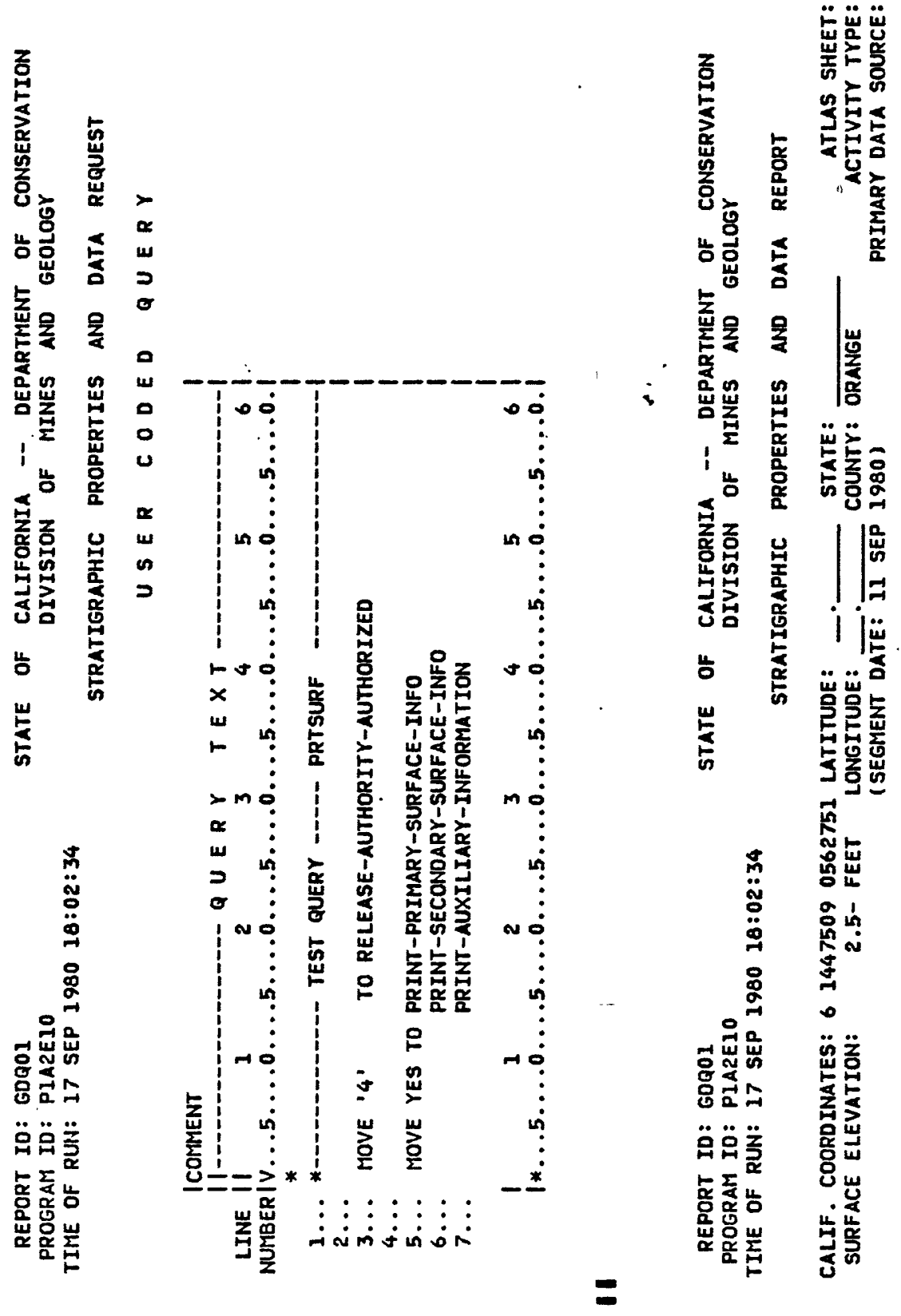

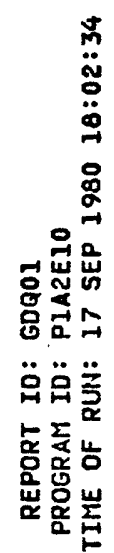



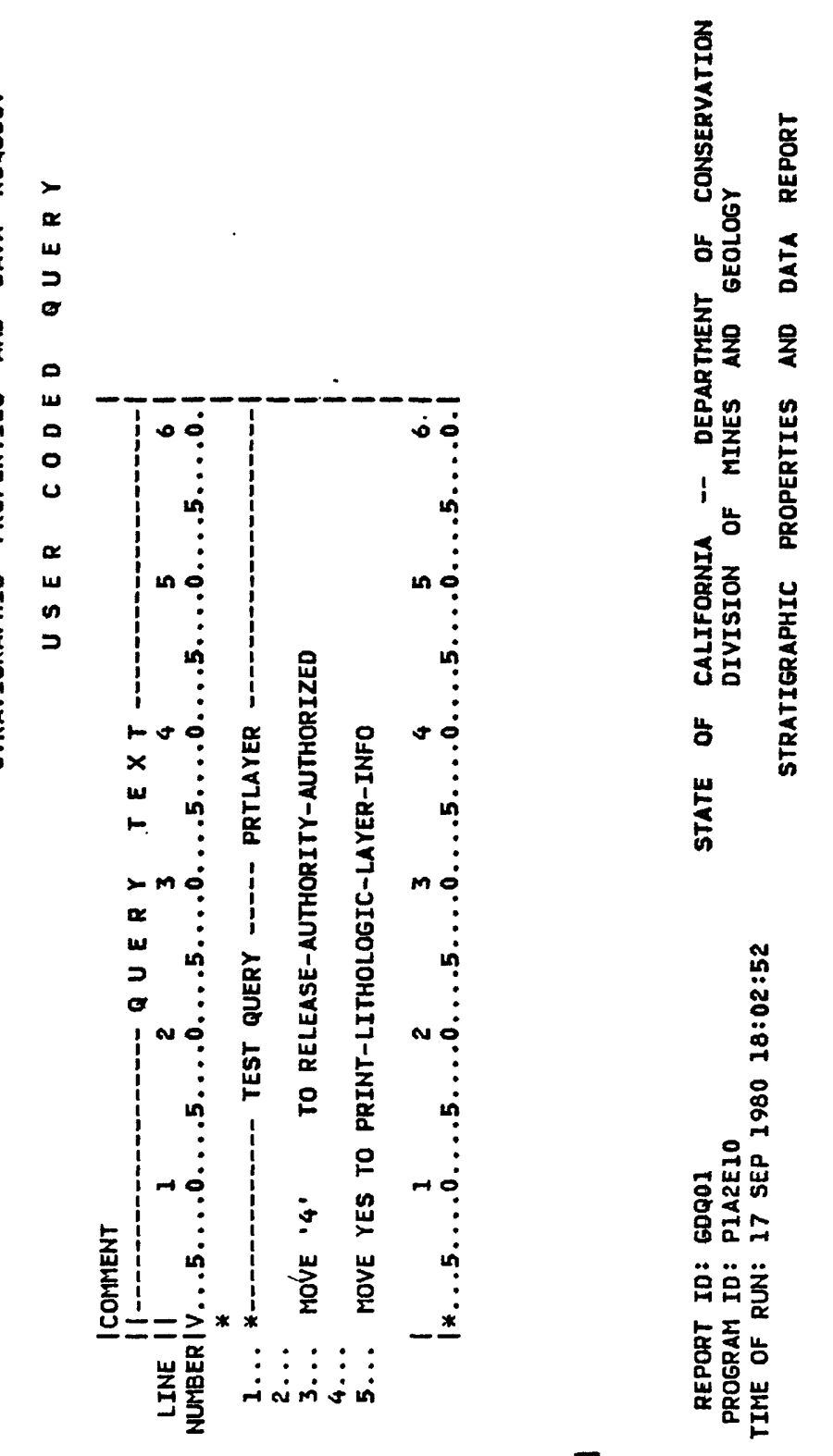

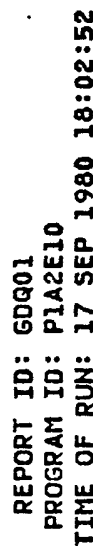

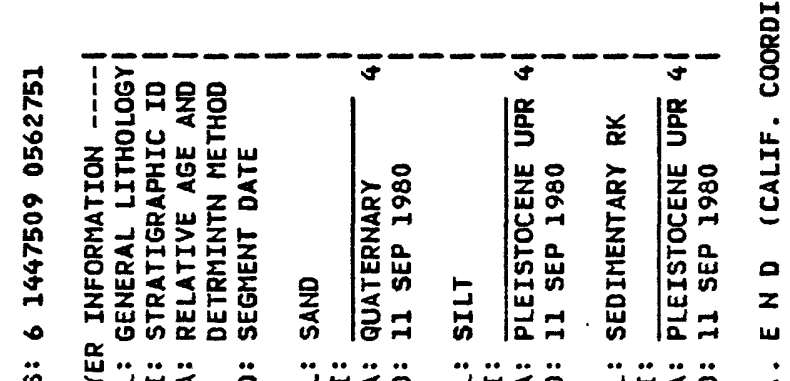

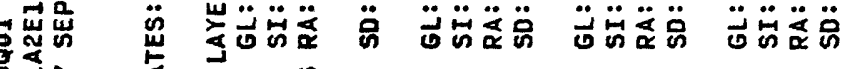

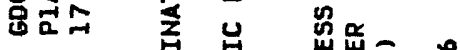
苗宾

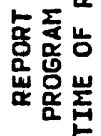
亭 언 울

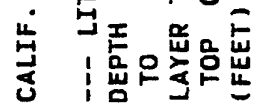
๑ 


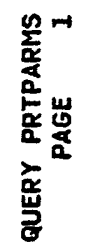
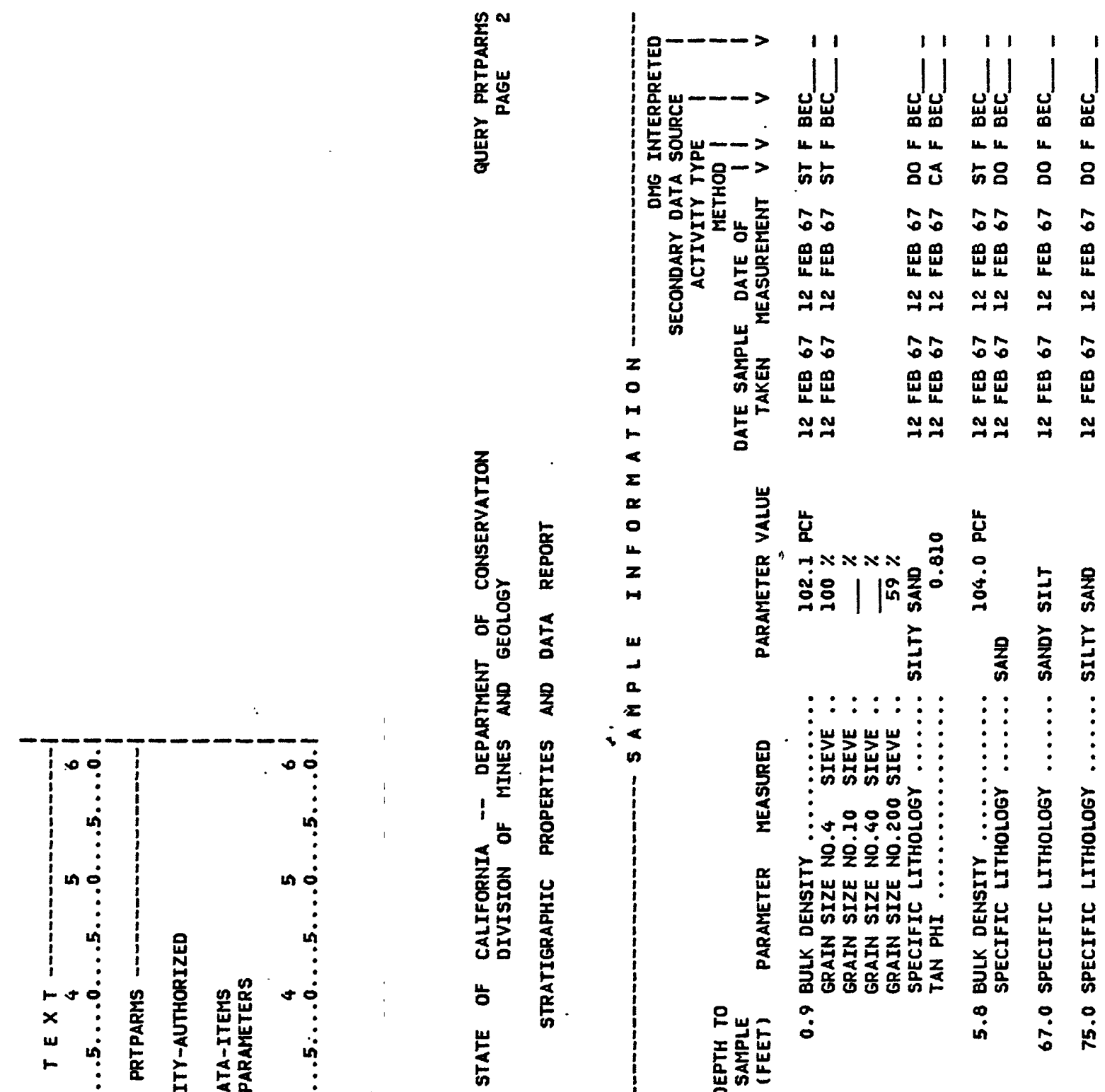

㝨是
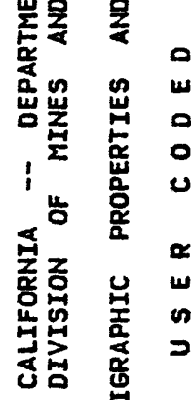

능

屴 $\approx$

홍
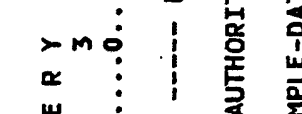

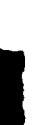

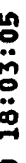

传

동 㟧

茄血

䓃唼

紫究这

㟔움논
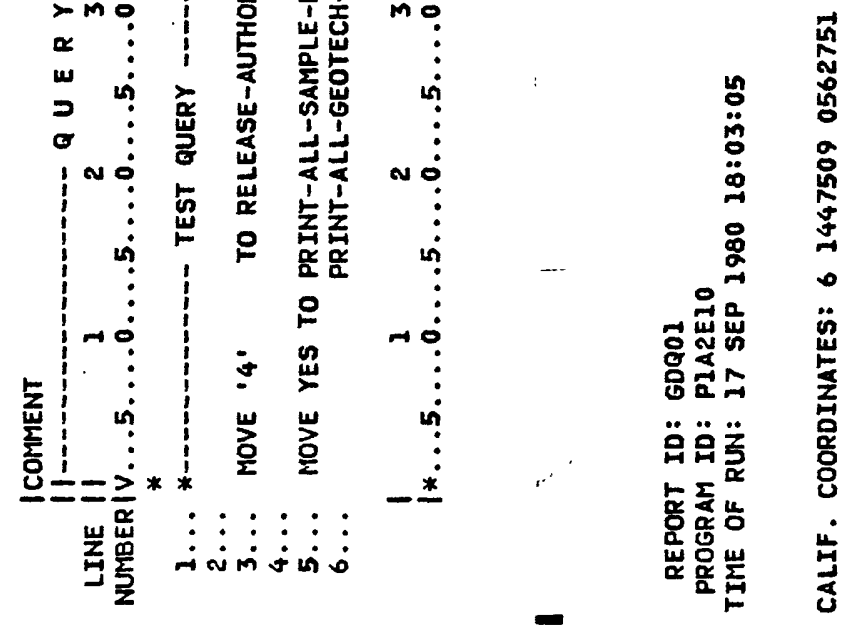

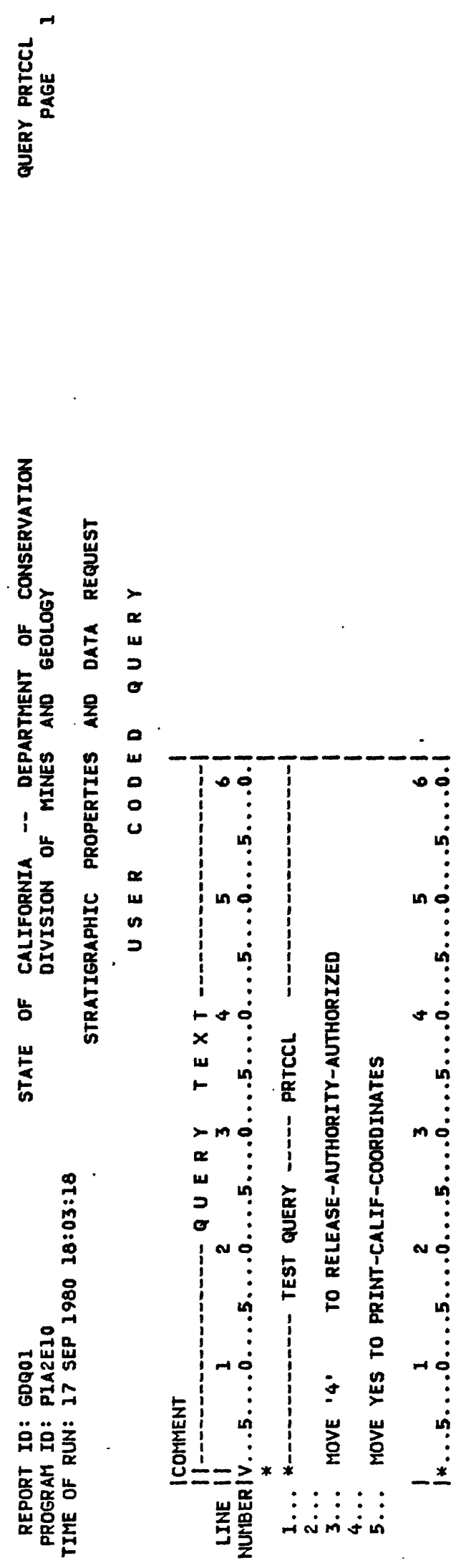


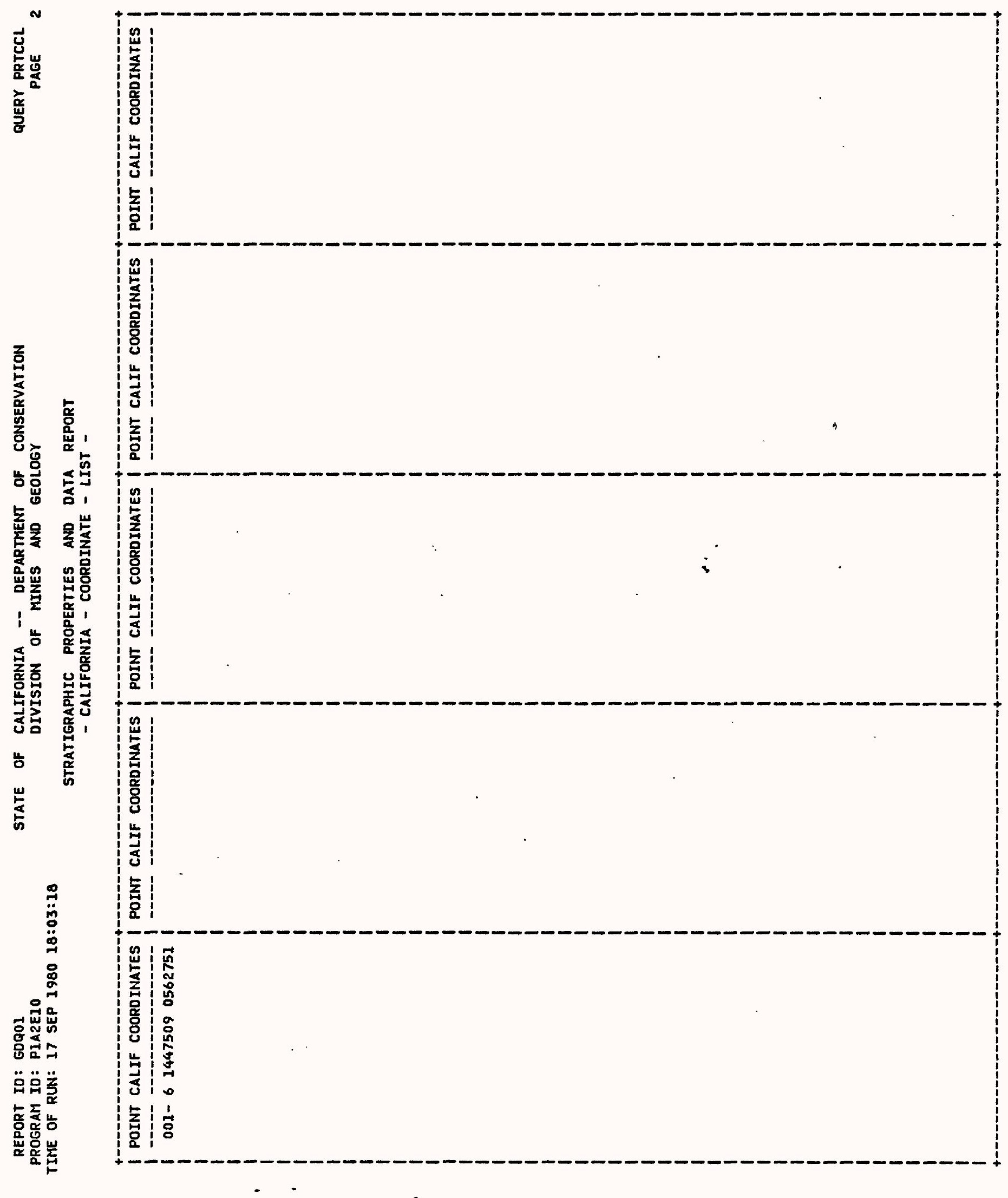



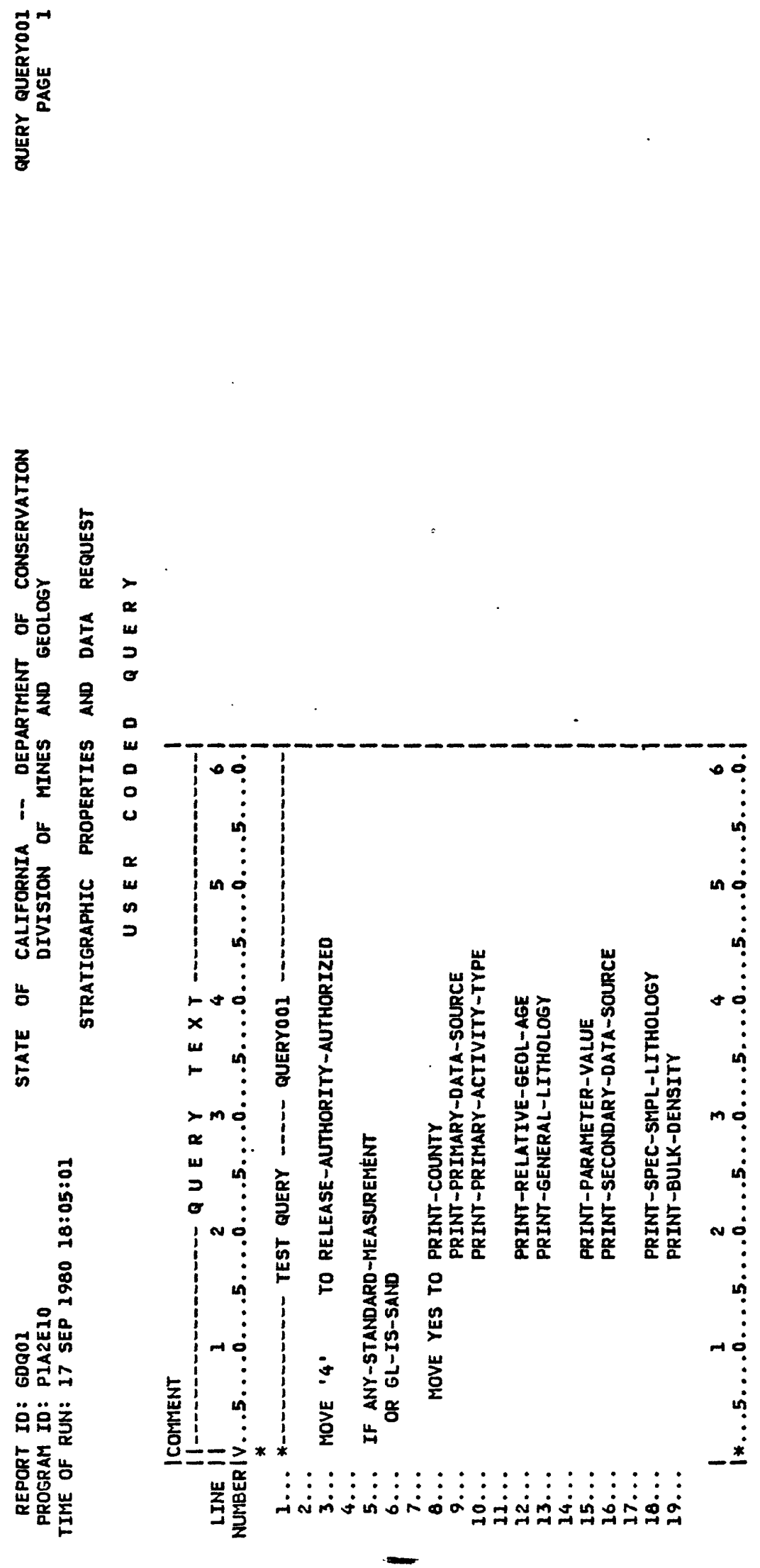

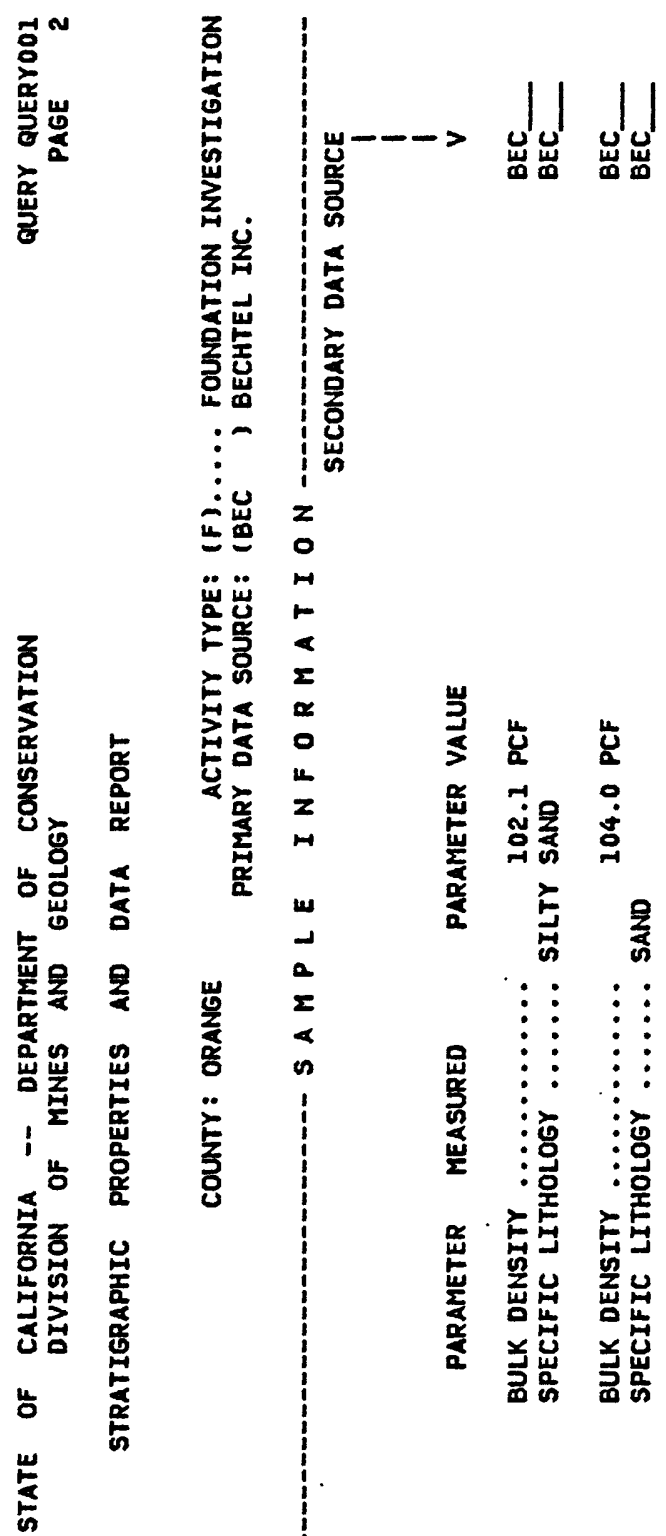

苨

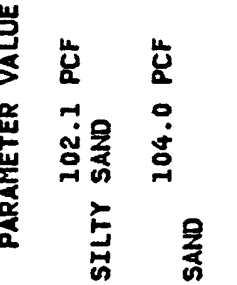

$\begin{array}{ll}\text { 岁 } & a \\ \frac{2}{5} & 0\end{array}$

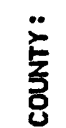

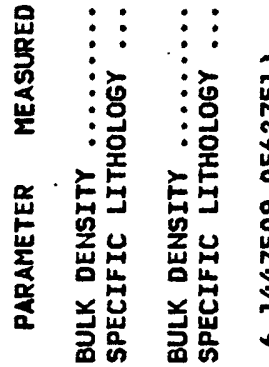

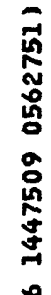

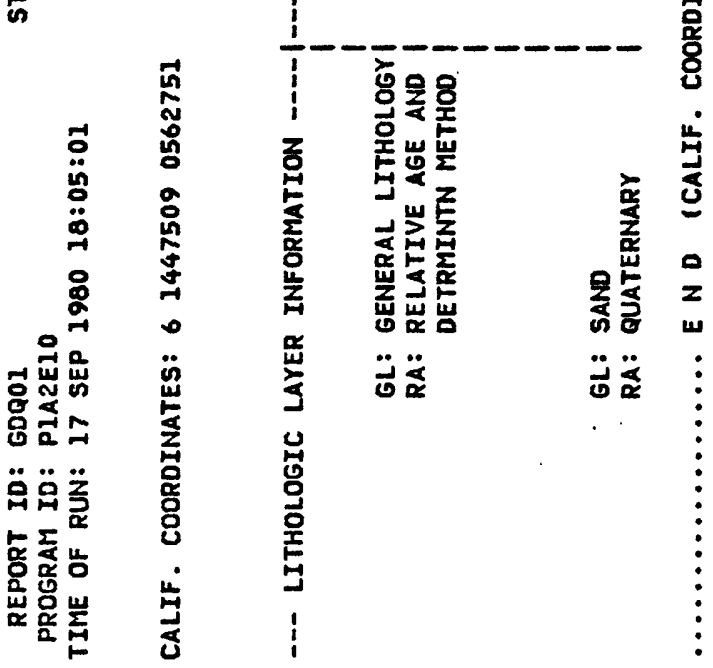




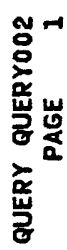

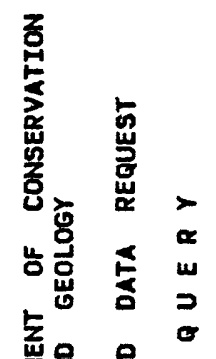

杽是是

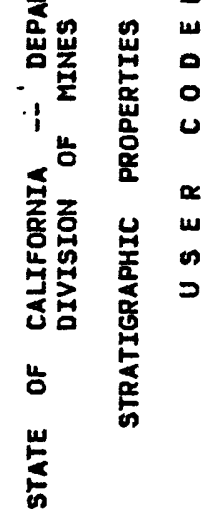

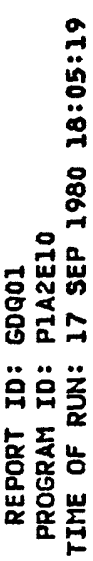

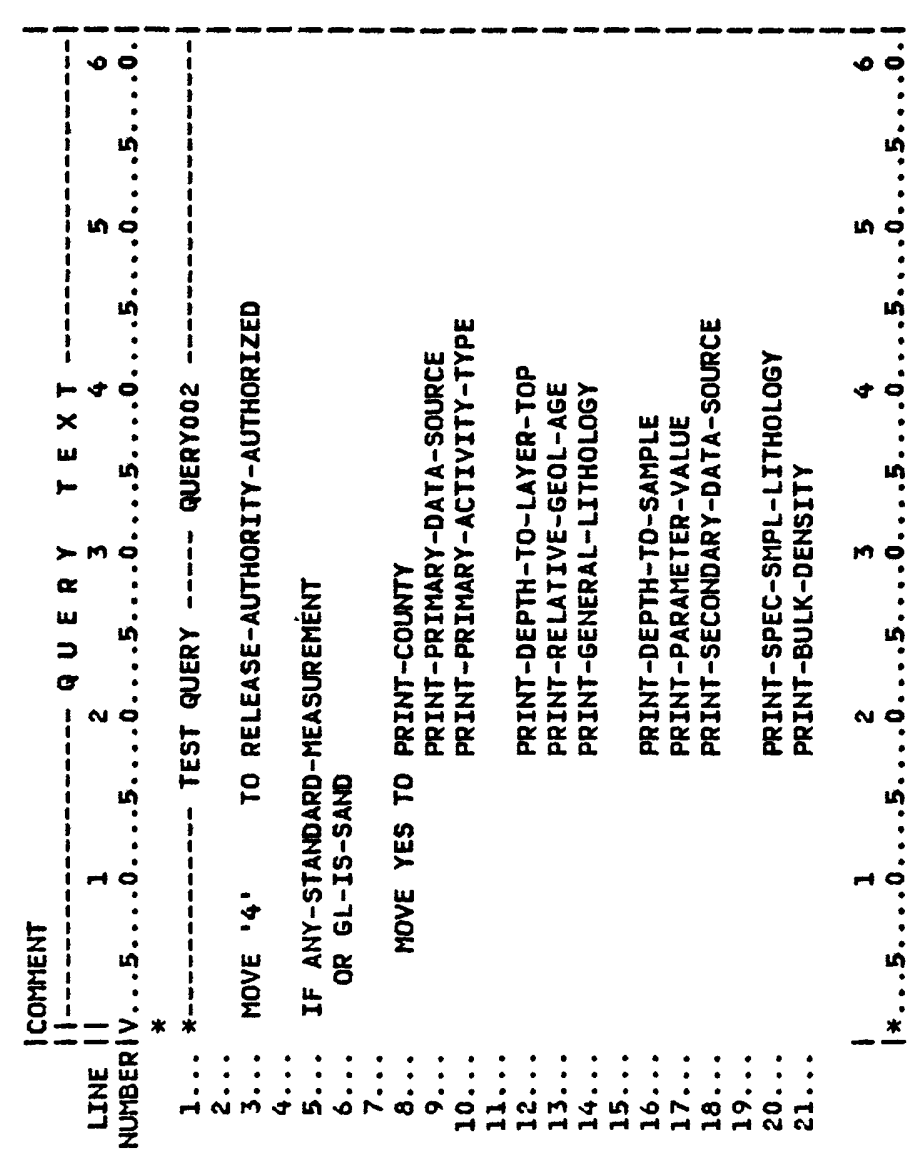




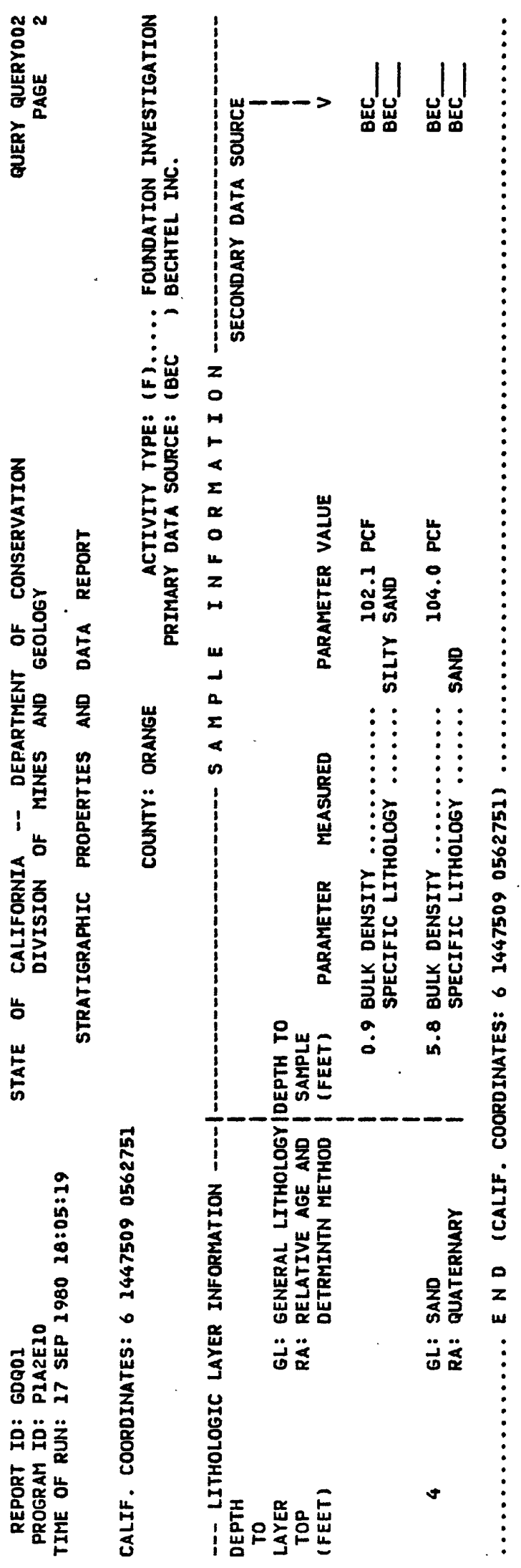



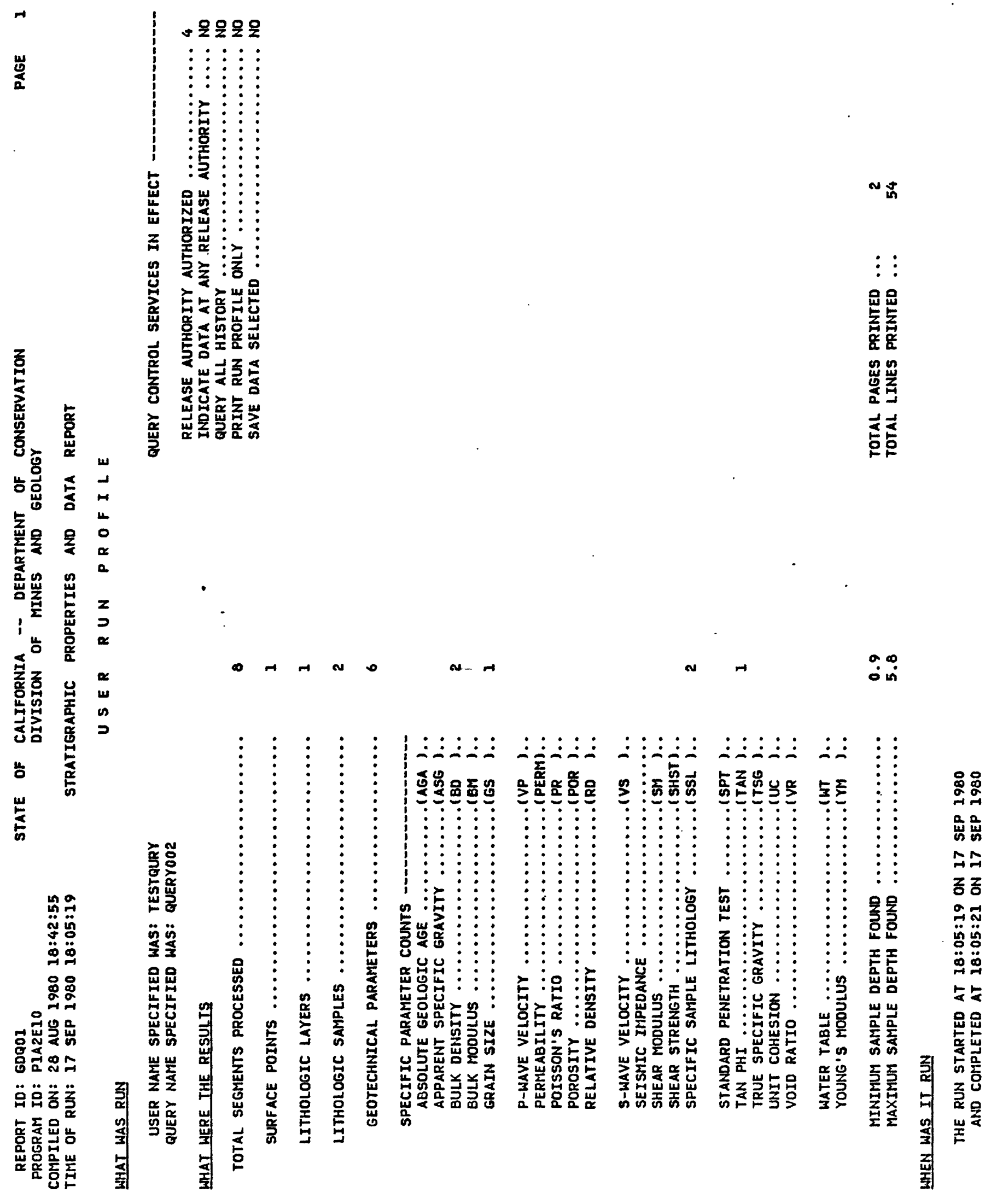

$\ddot{0}$ 


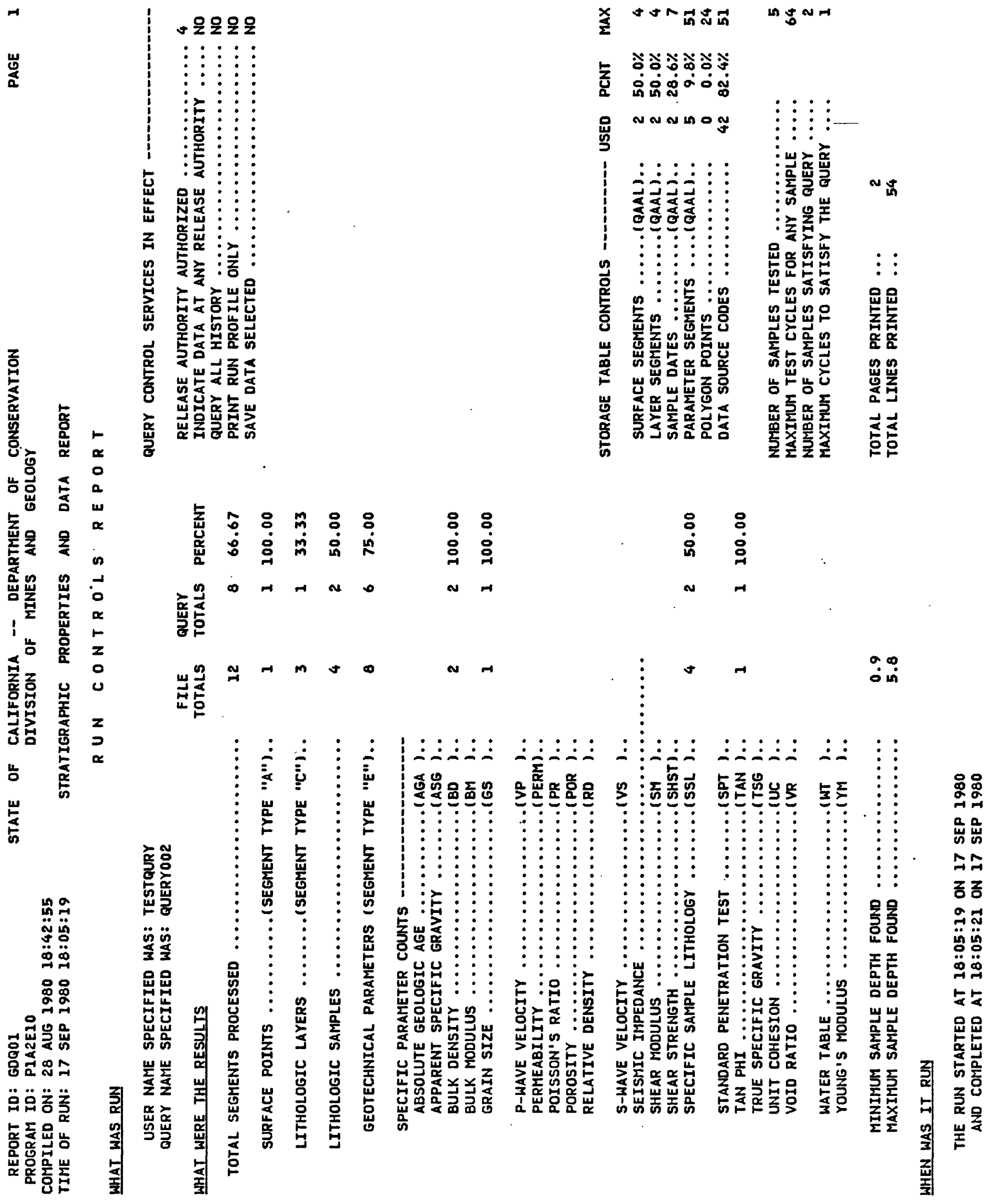



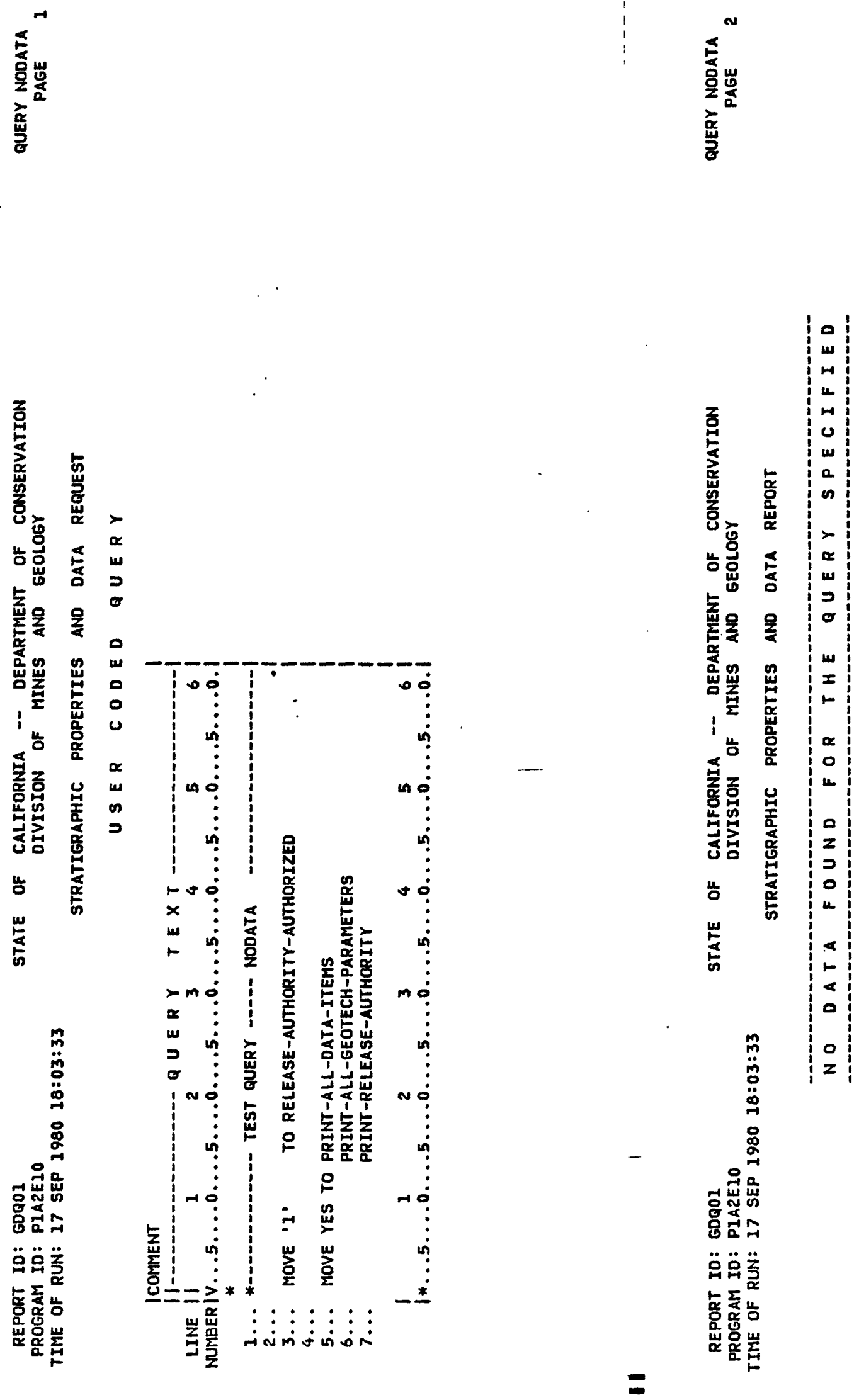

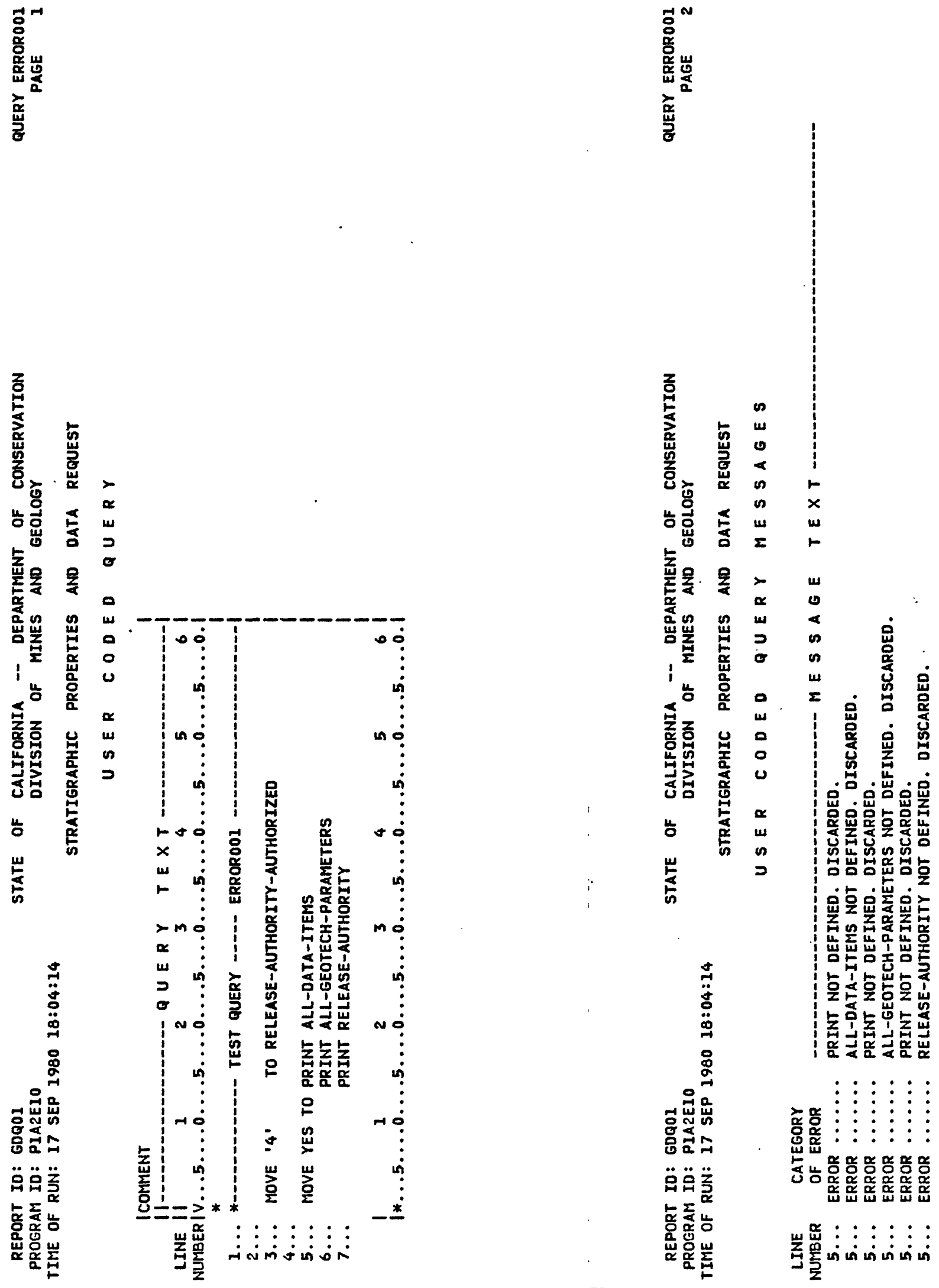

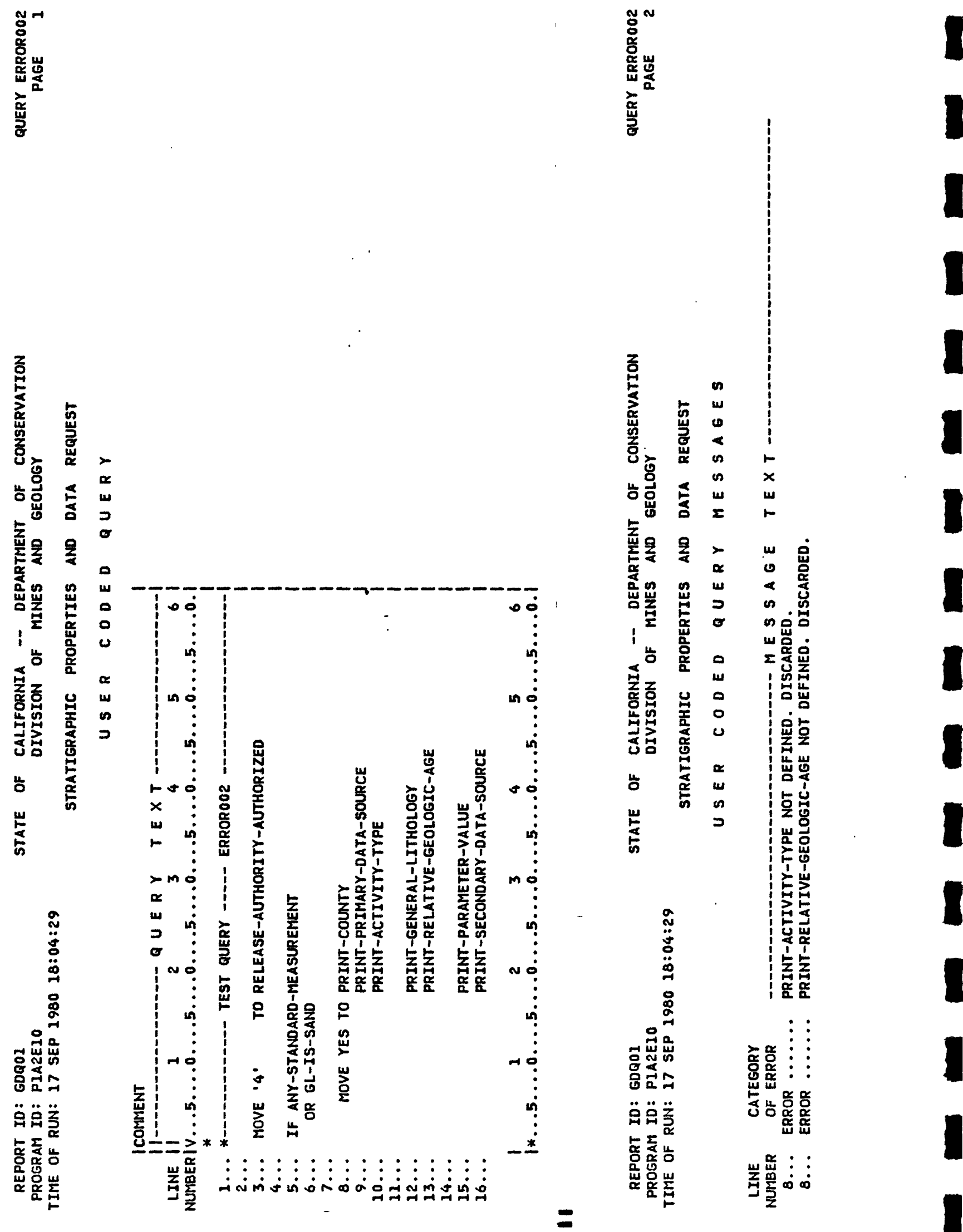

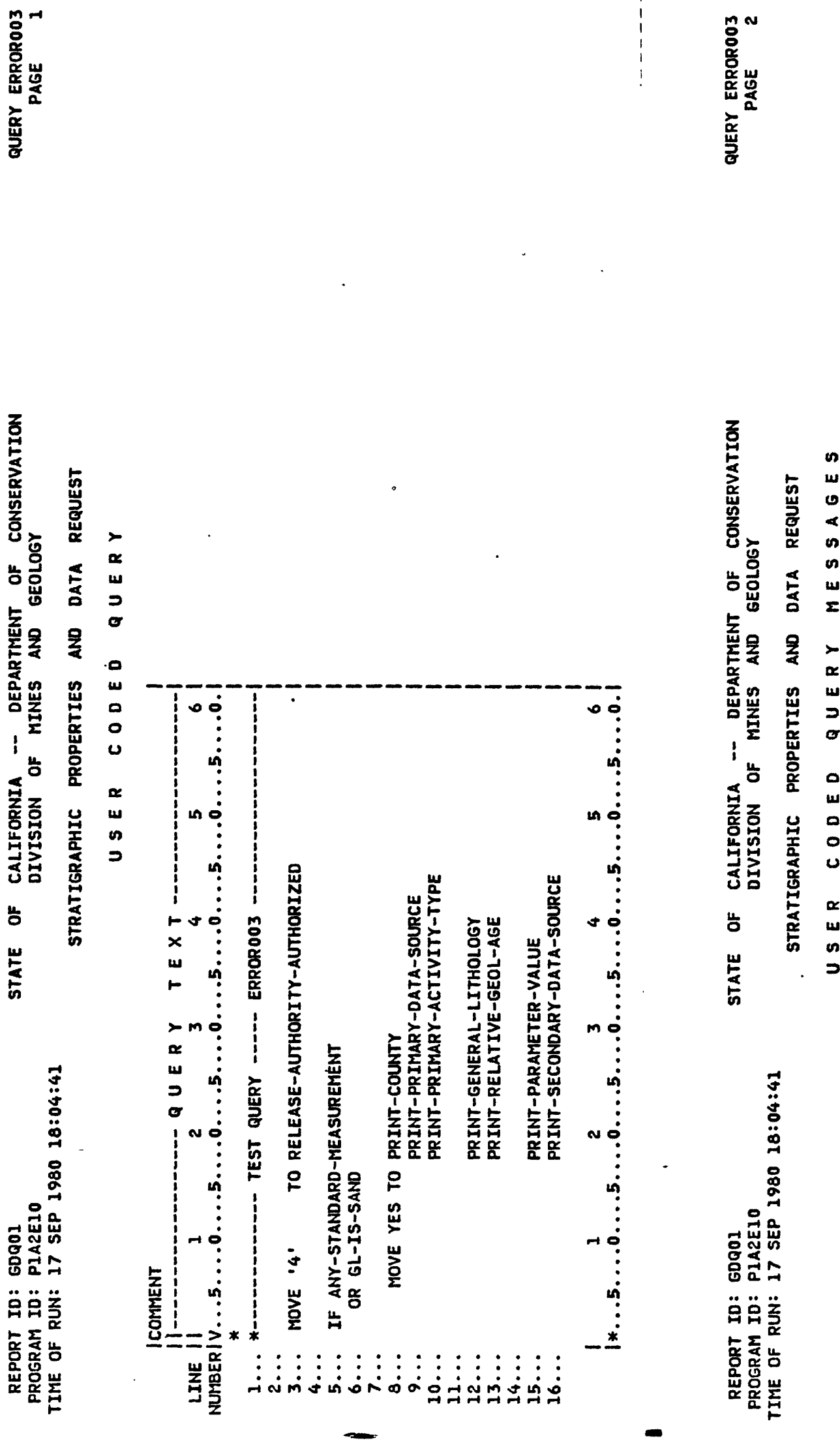


\section{ADDITIONAL QUERY EXAMPLES}

QUERY

NAME

PRTAVAIL

PRINTCCL

DEMO01

DEMO01A

DEMO03

DEMO03A

DEMOO3B

DEMOO3C
Query examples from a demonstration storage file--

1) Two pages from a report of all data available showing all data item values on file for one ?." hole.

2) A request for a 1 ist of all California Coordinate values on file.

3) A request in which the condition parameters are varied.

a) depth to sample greater than 100 feet

b) depth to sample greater than 35 feet pluS USER RUN PROFILE report

4) A request in which the presentation or printing parameters are varied.

a) print without DEPTH TO LAYER TOP

b) print with DEPTH TO LAYER TOP

c) print California Coordinates only

d) request for parameters for which no data is on file plus the USER RUN PROFILE report 


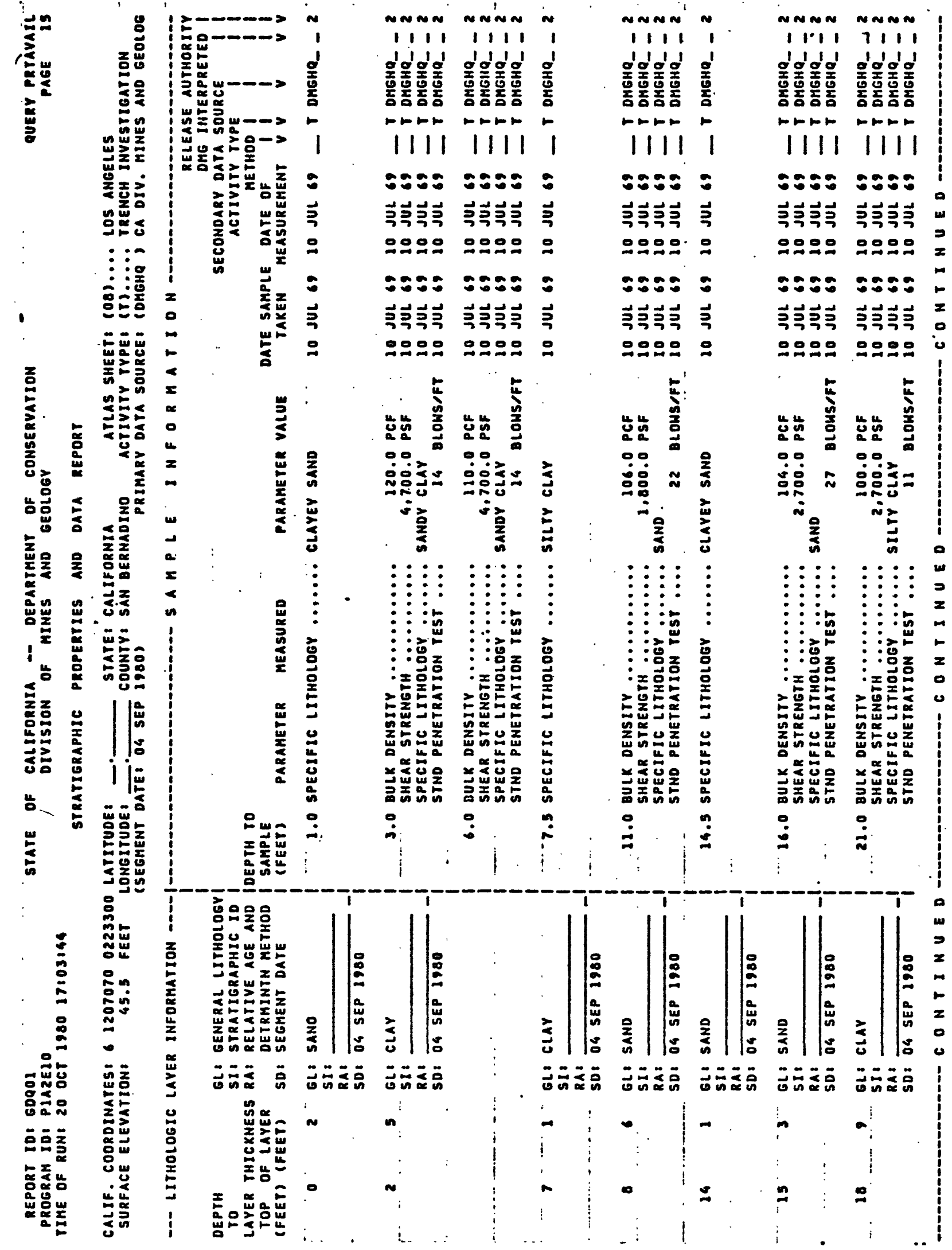




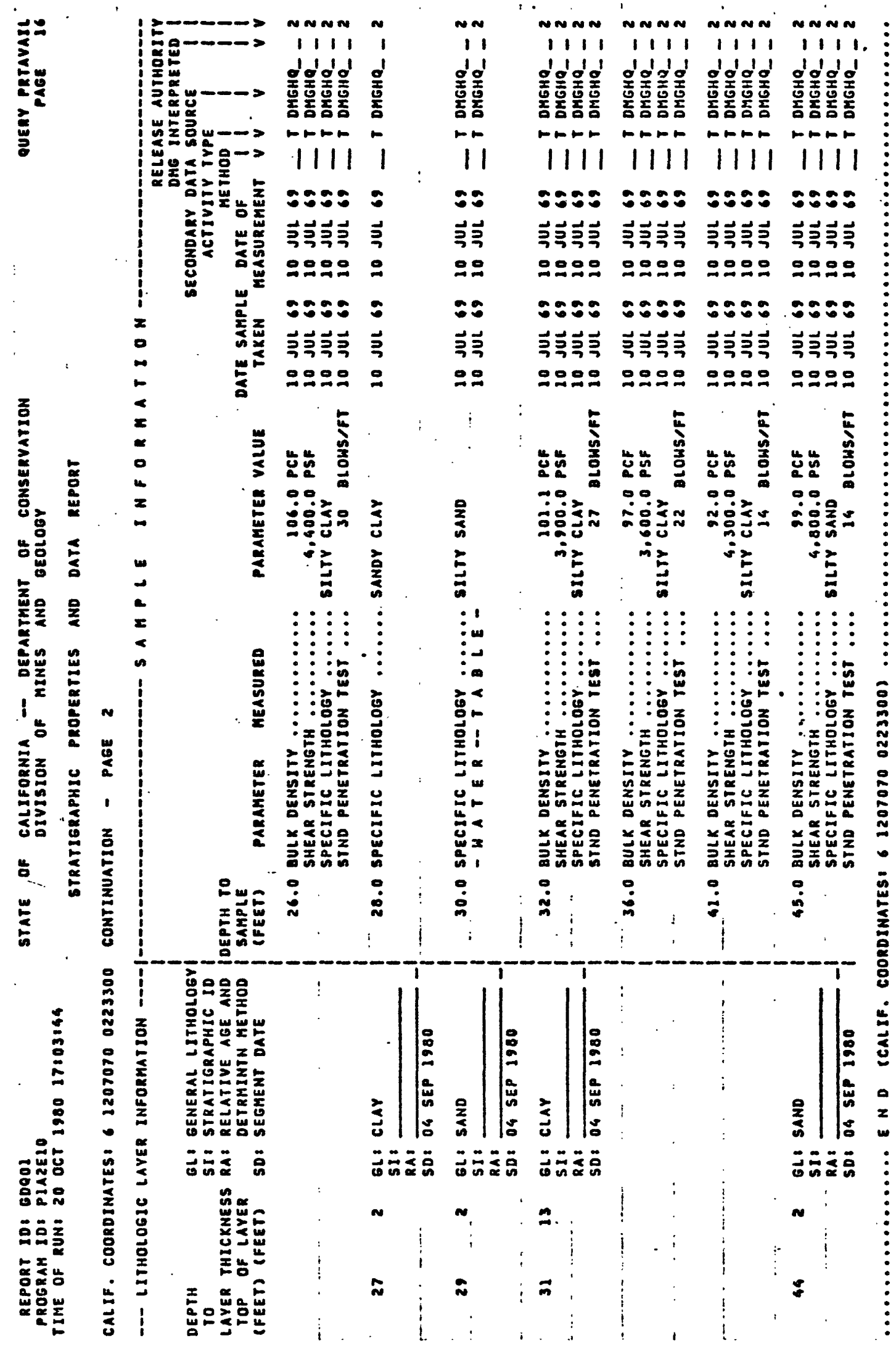



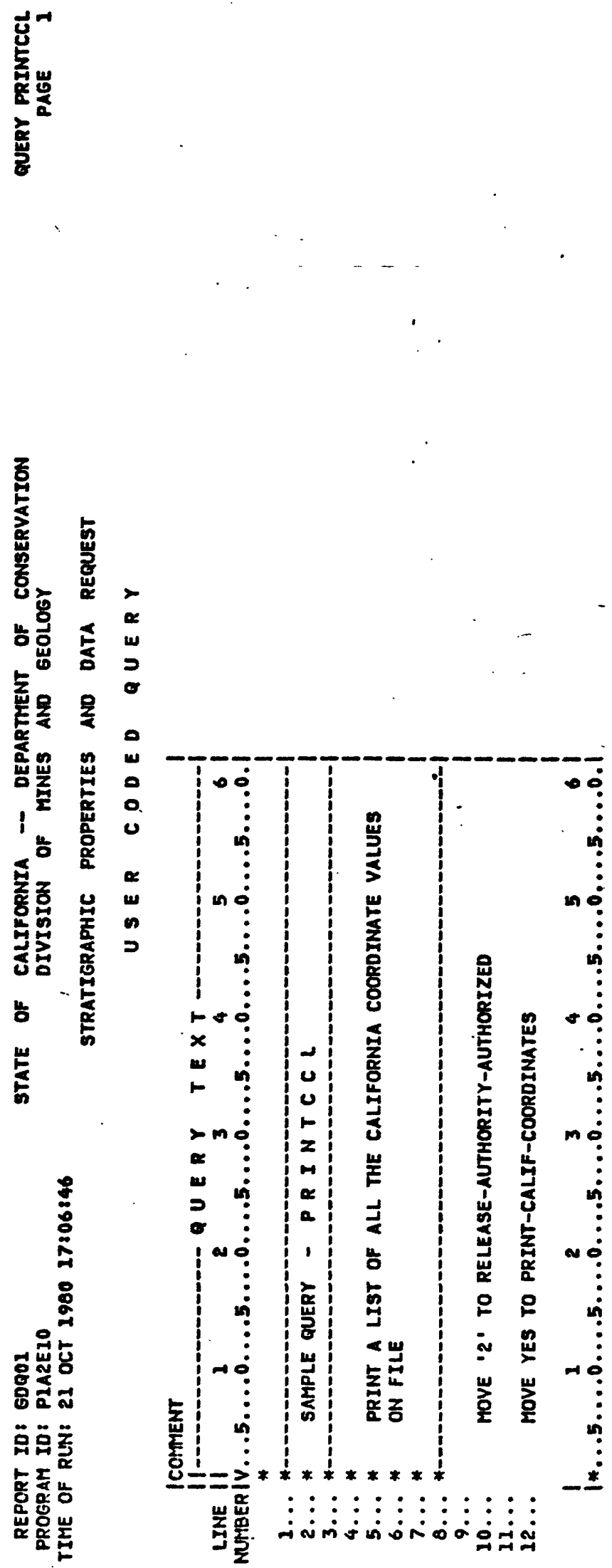


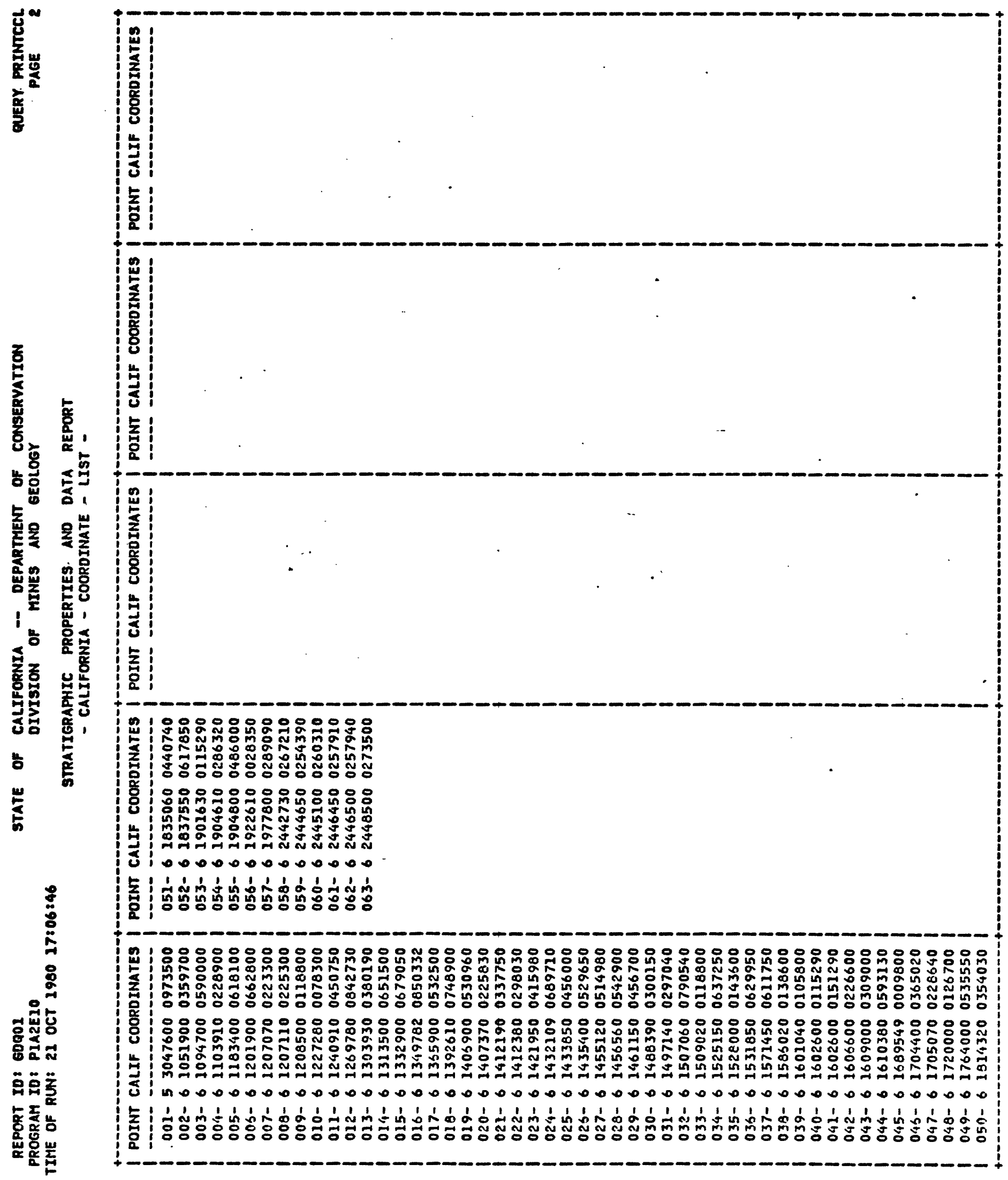


通望

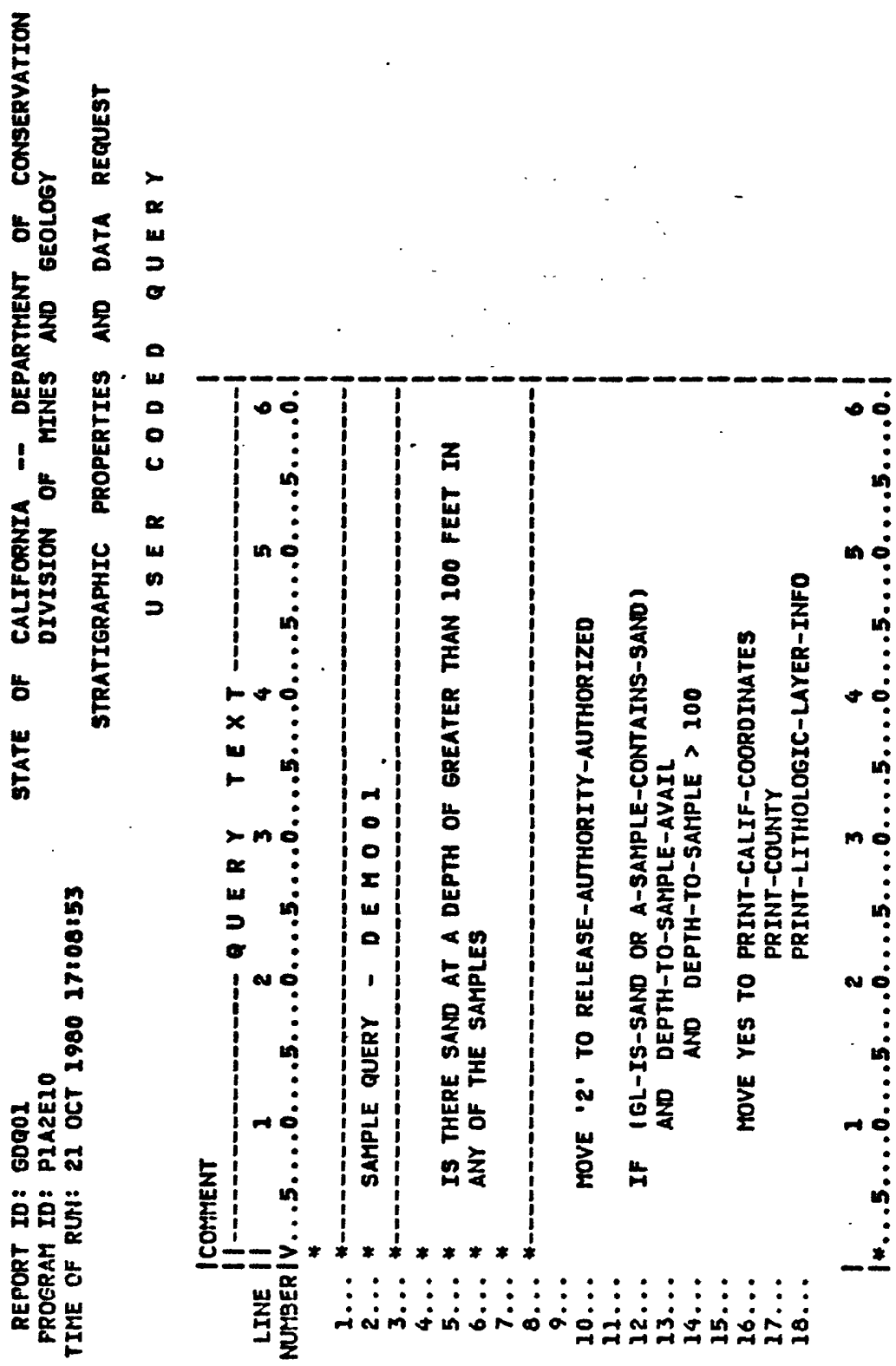




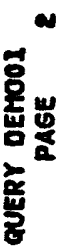

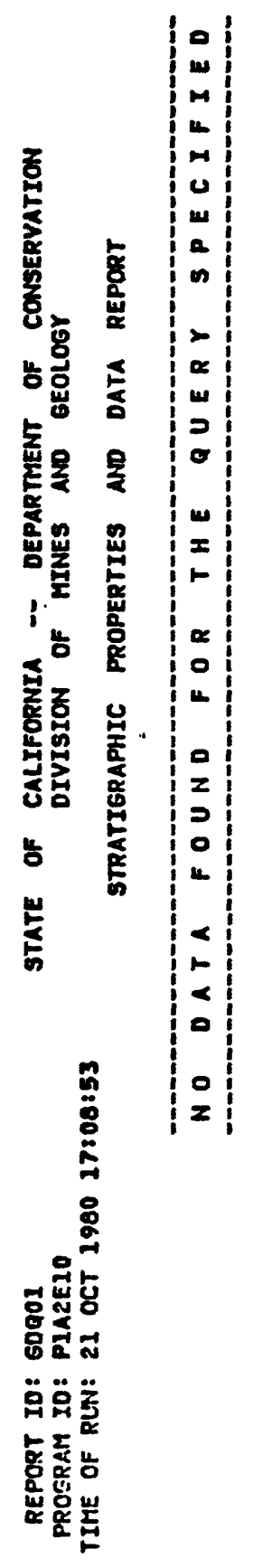



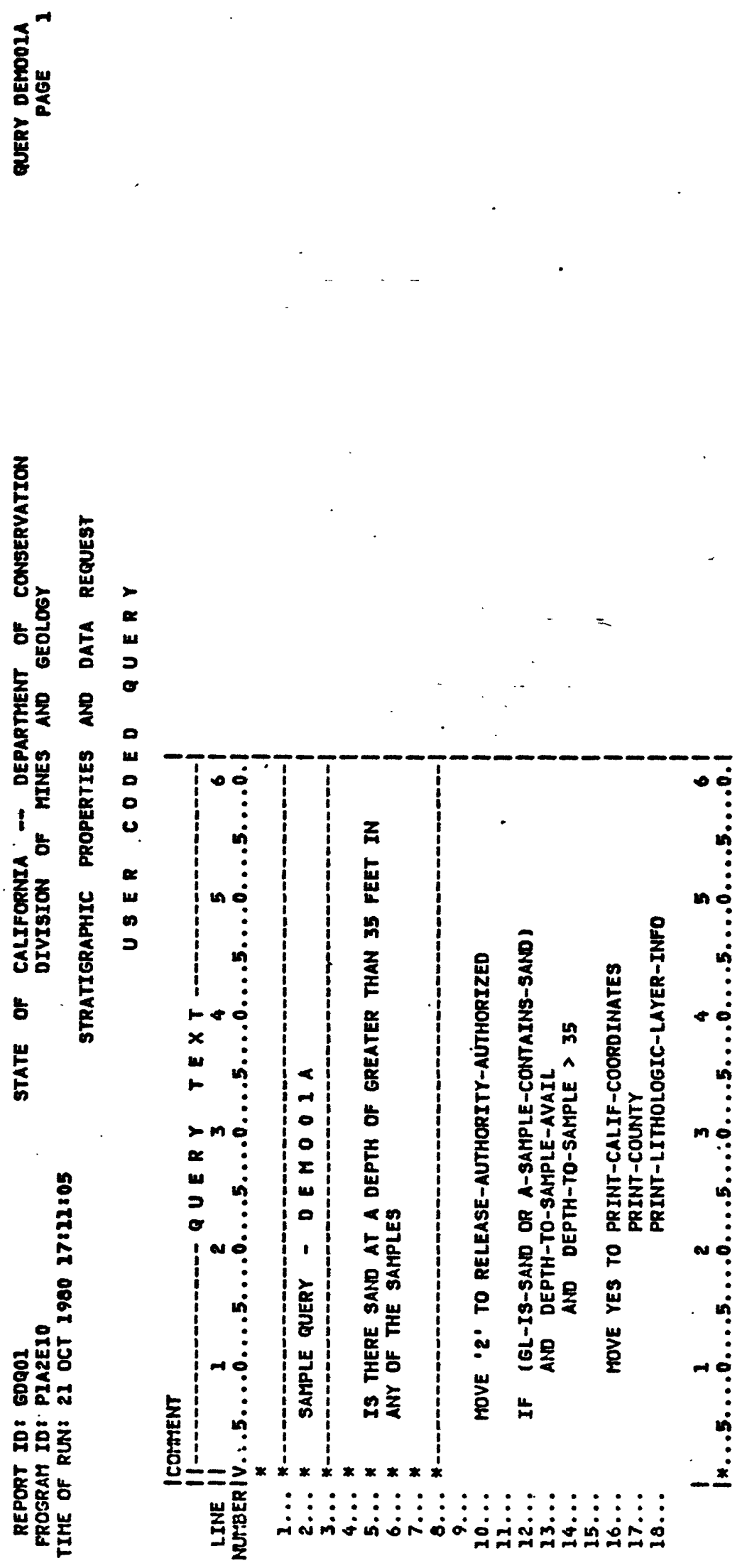

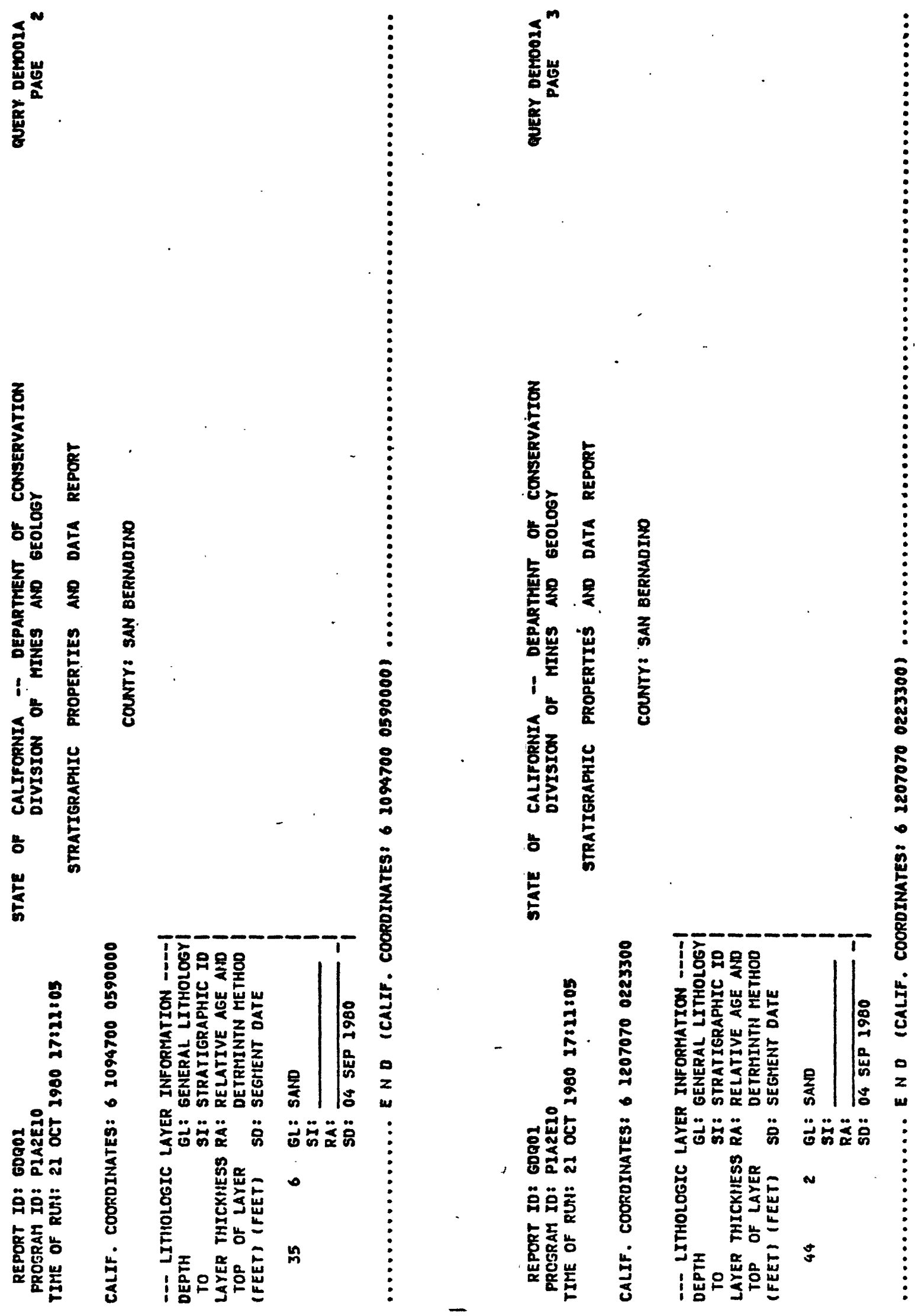

$$
\begin{aligned}
& \text { 茷 } \\
& \text { 孞 }
\end{aligned}
$$



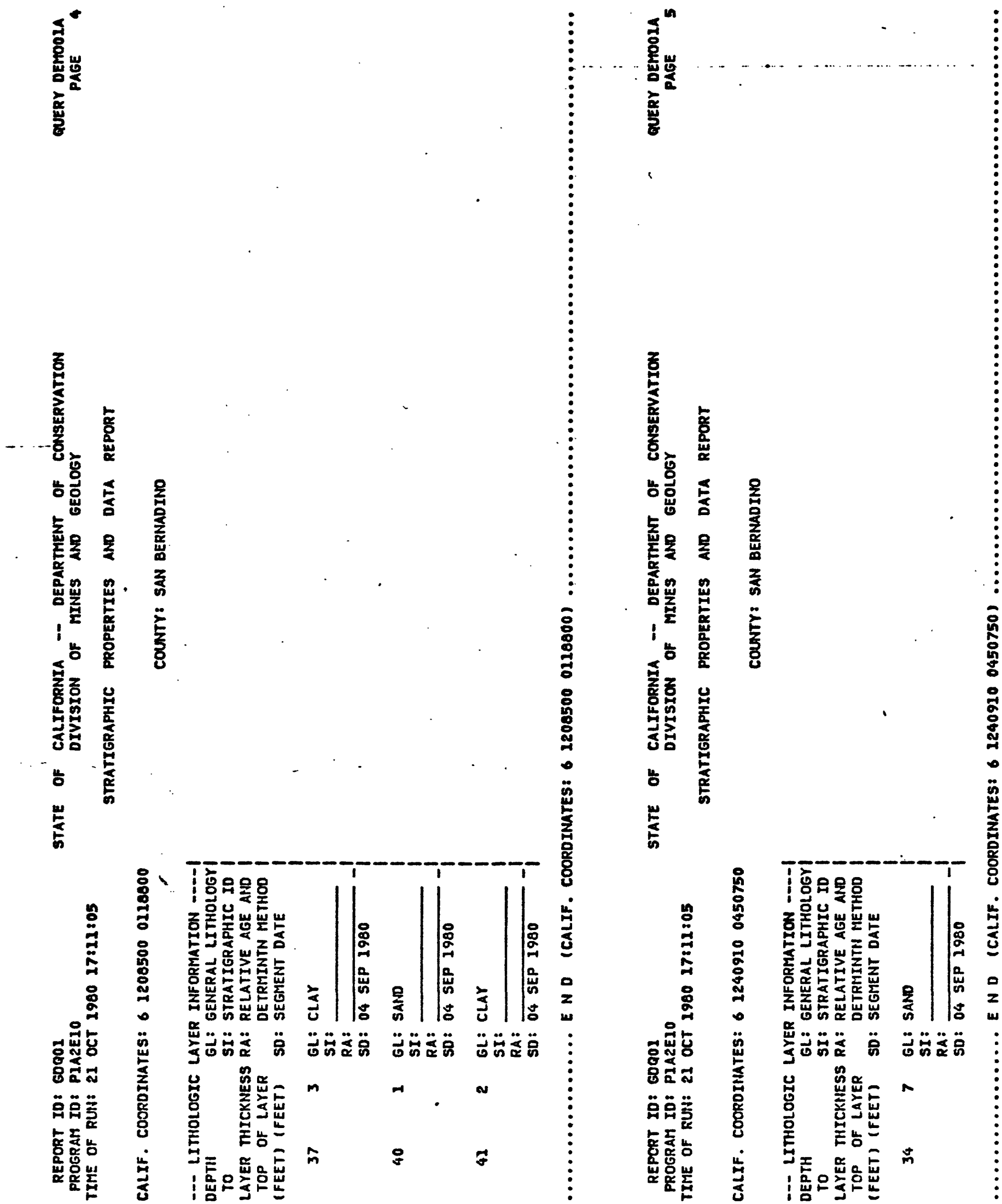

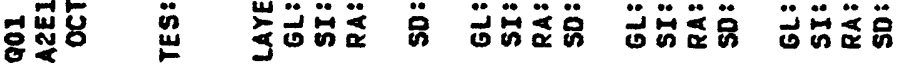

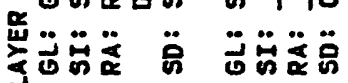

可言

整产

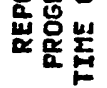
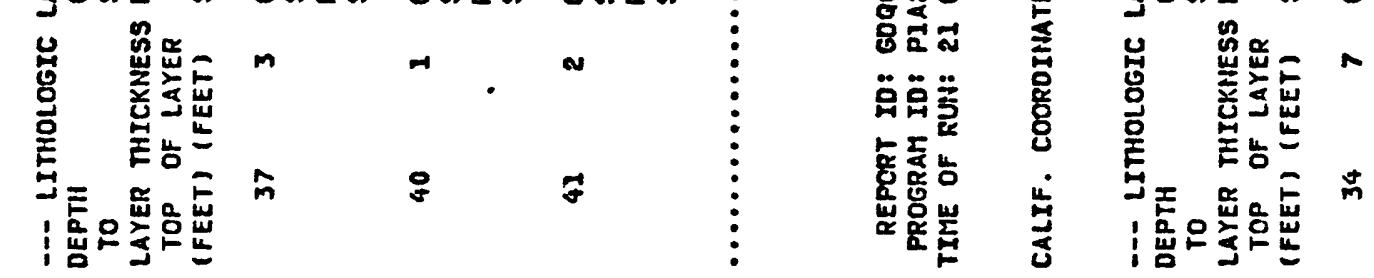

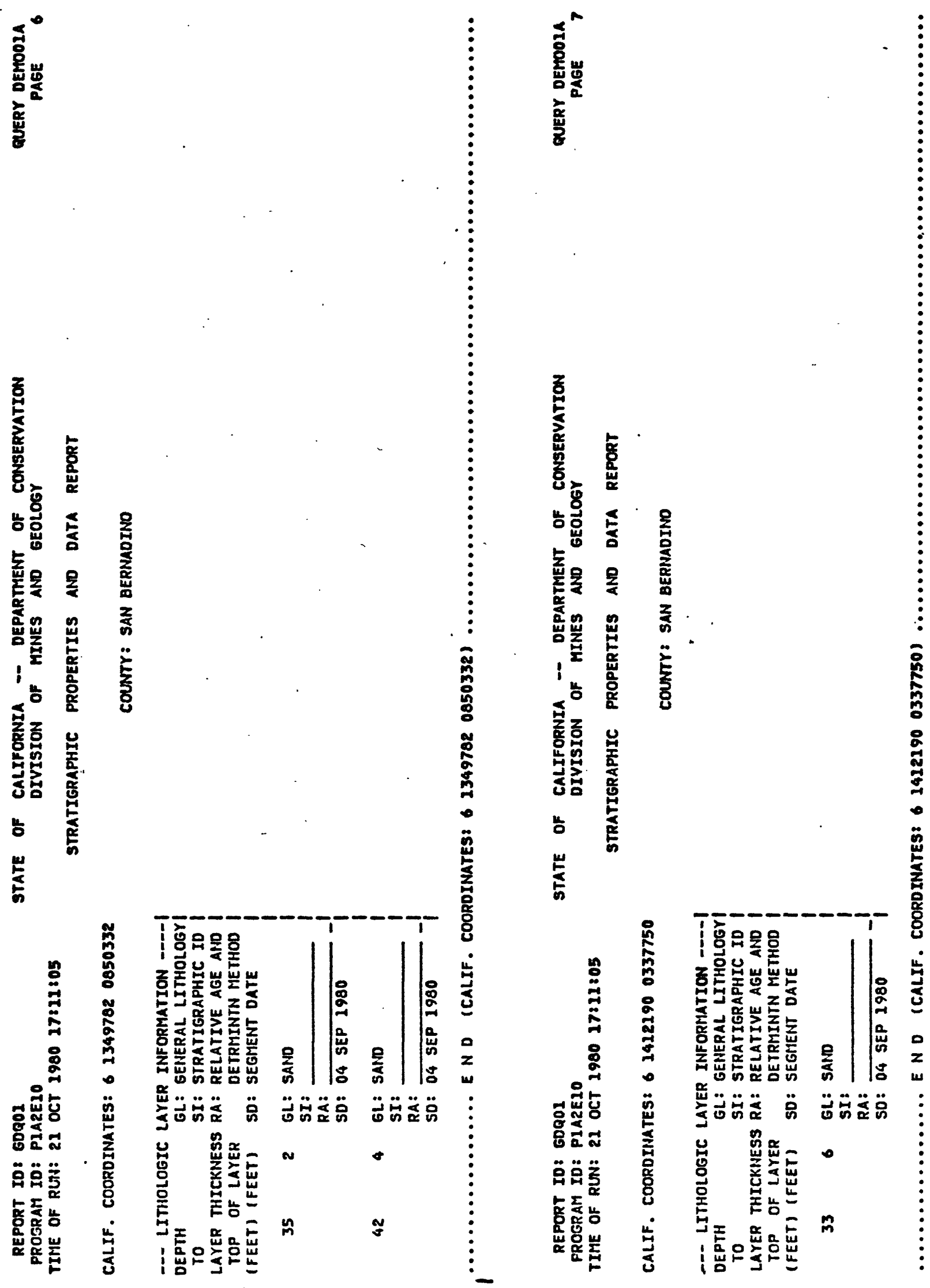

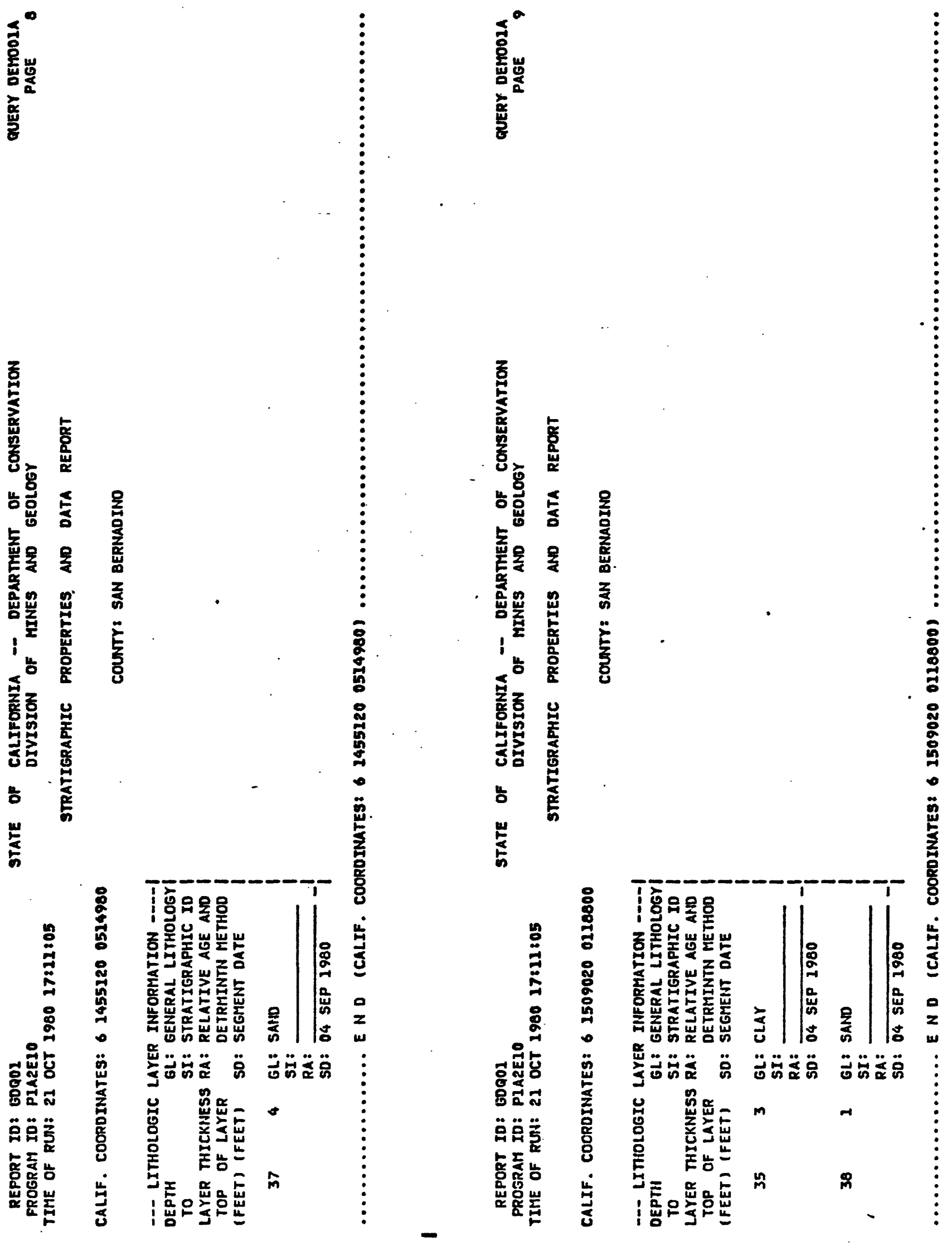

总

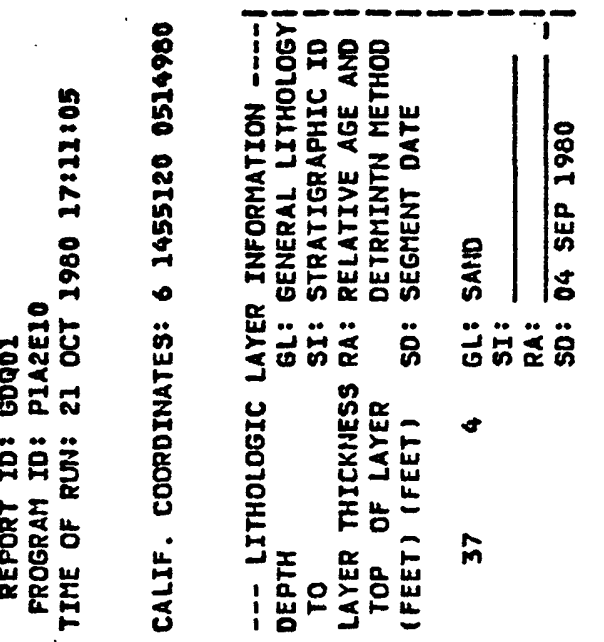


总㟧

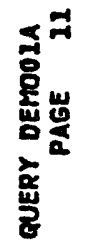

疍

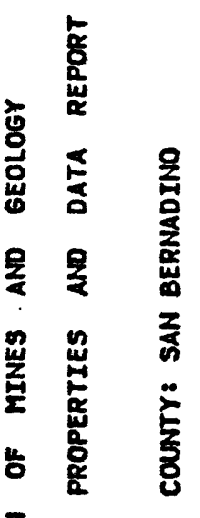

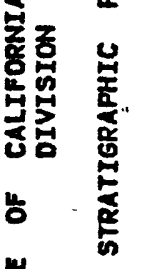

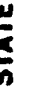

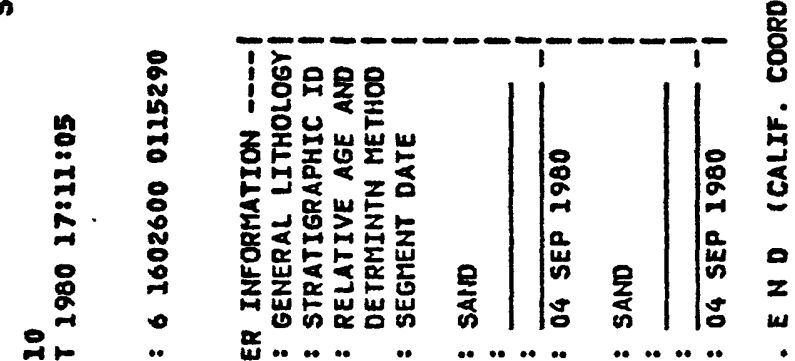

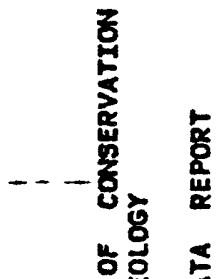

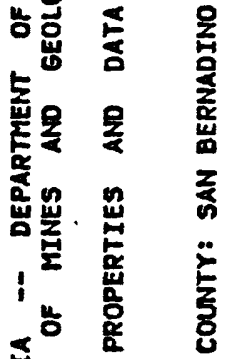

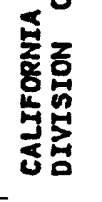

\%

䓂

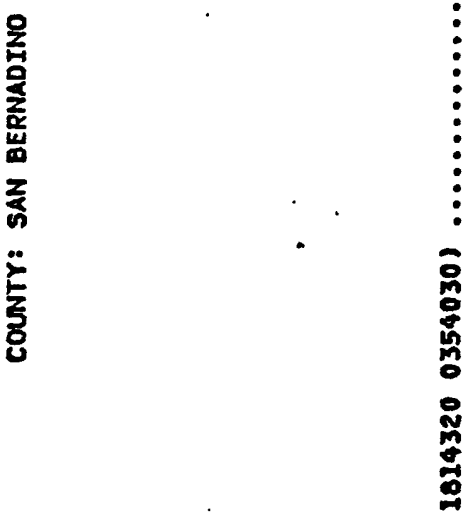

$\underset{5}{\frac{w}{5}}$

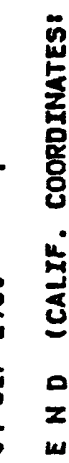

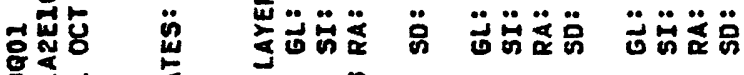

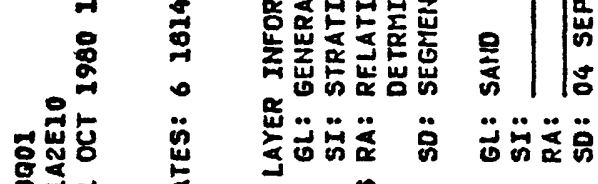

这苂ส

草苛

唯登。

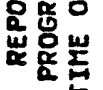

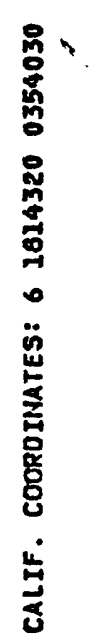

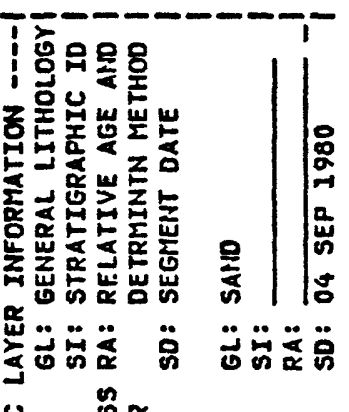

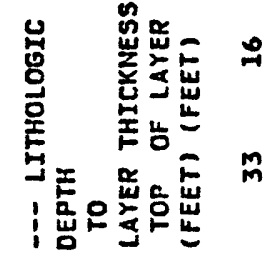



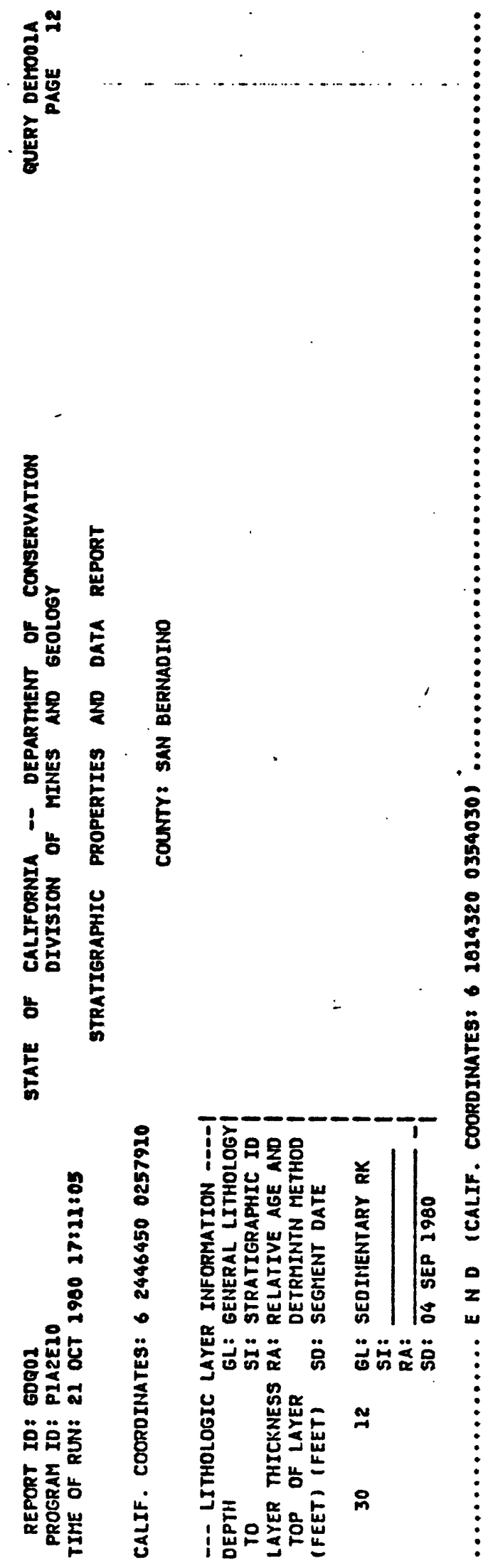


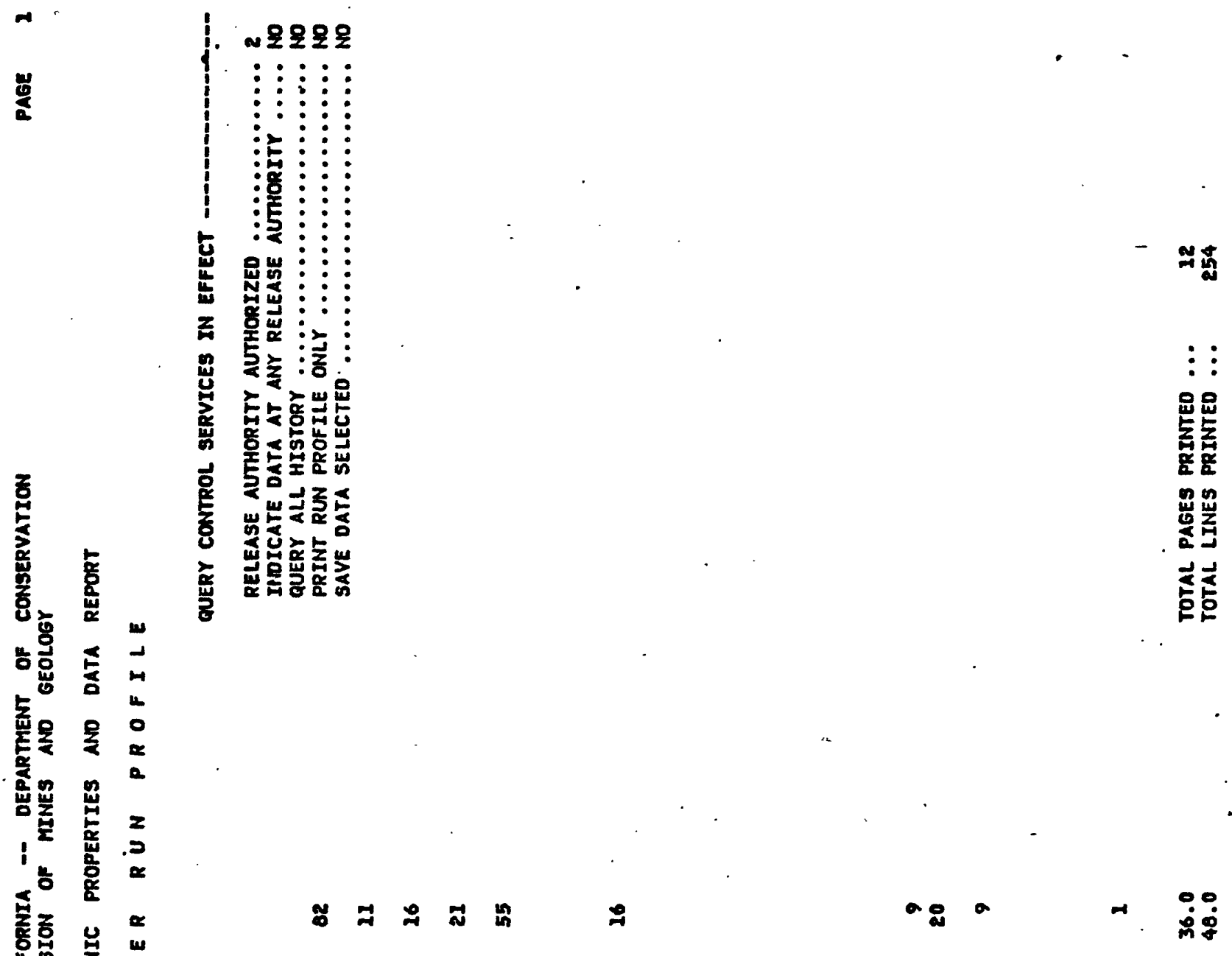




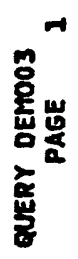

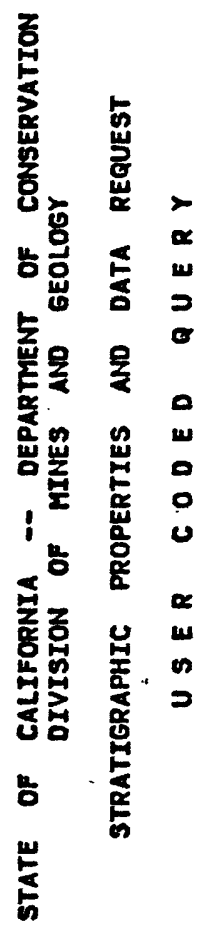

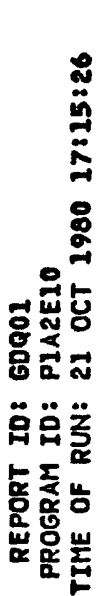

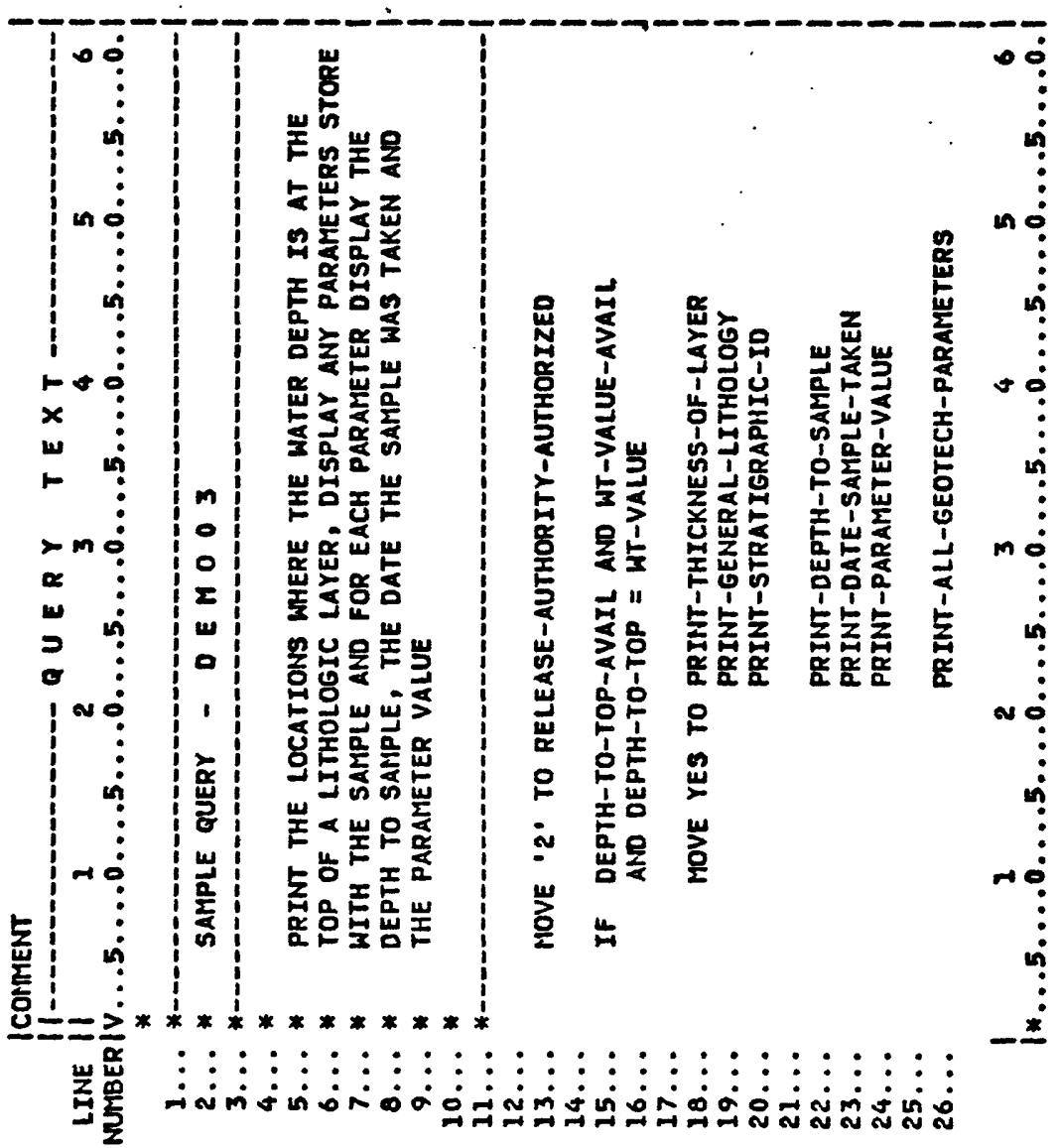




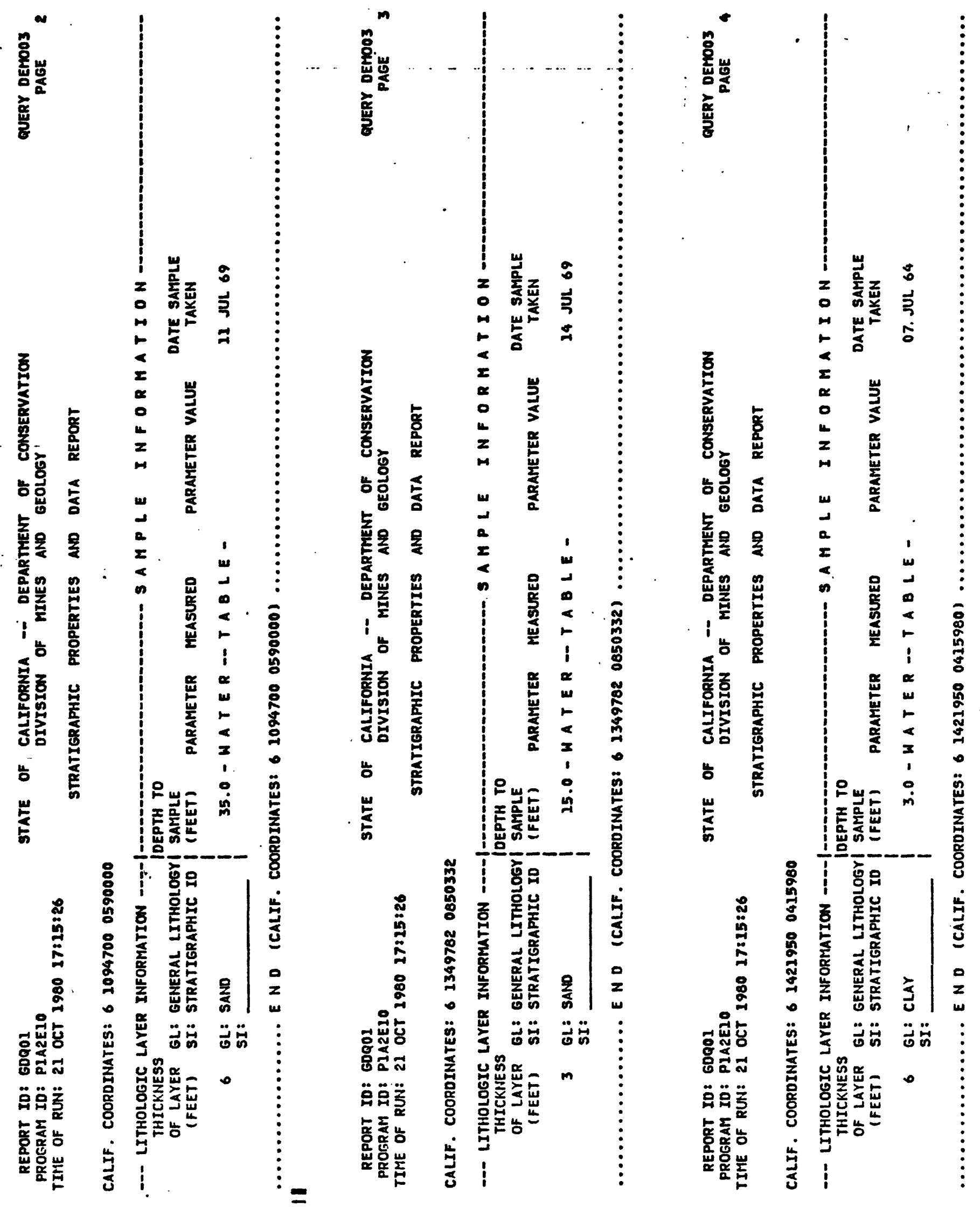




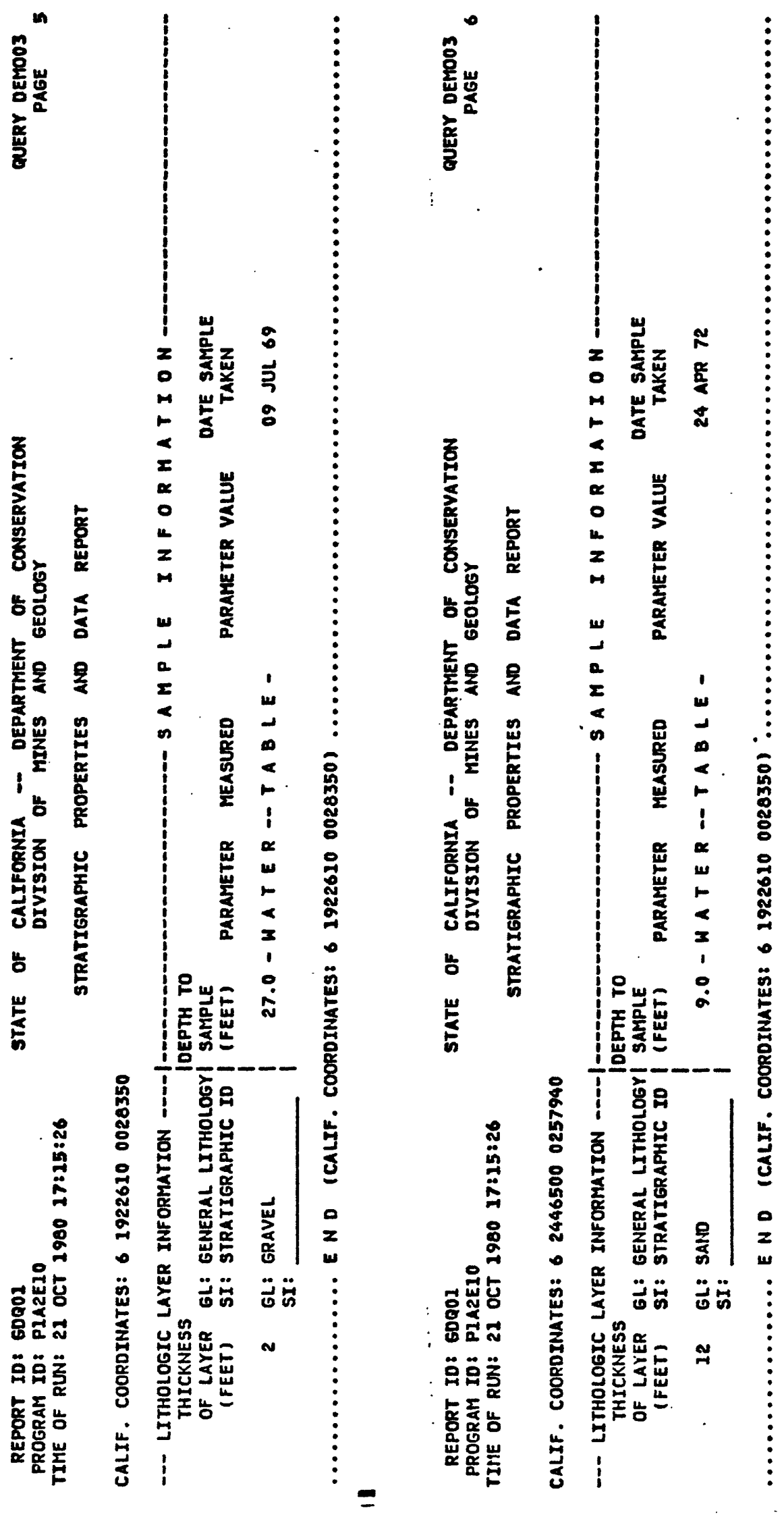




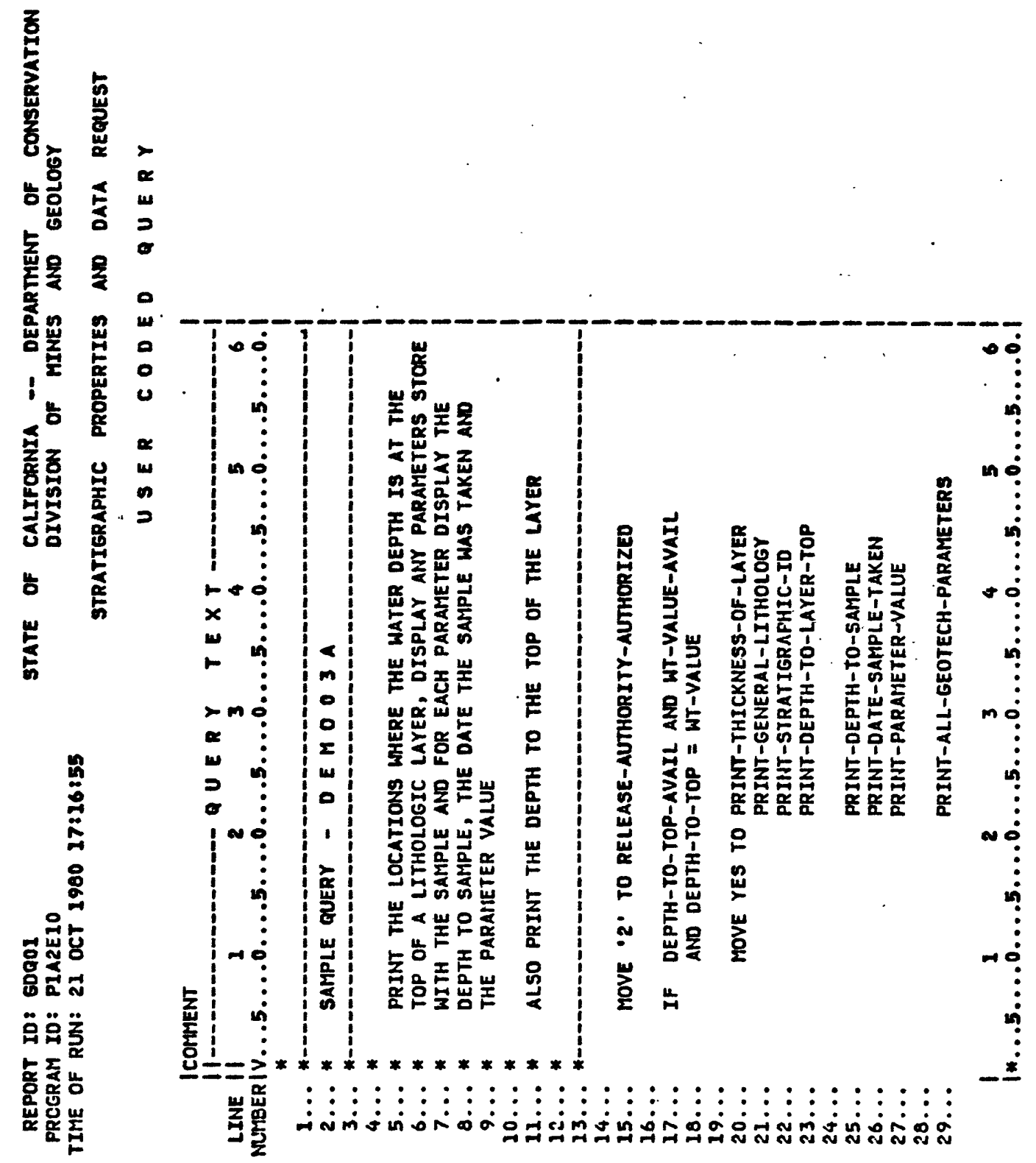



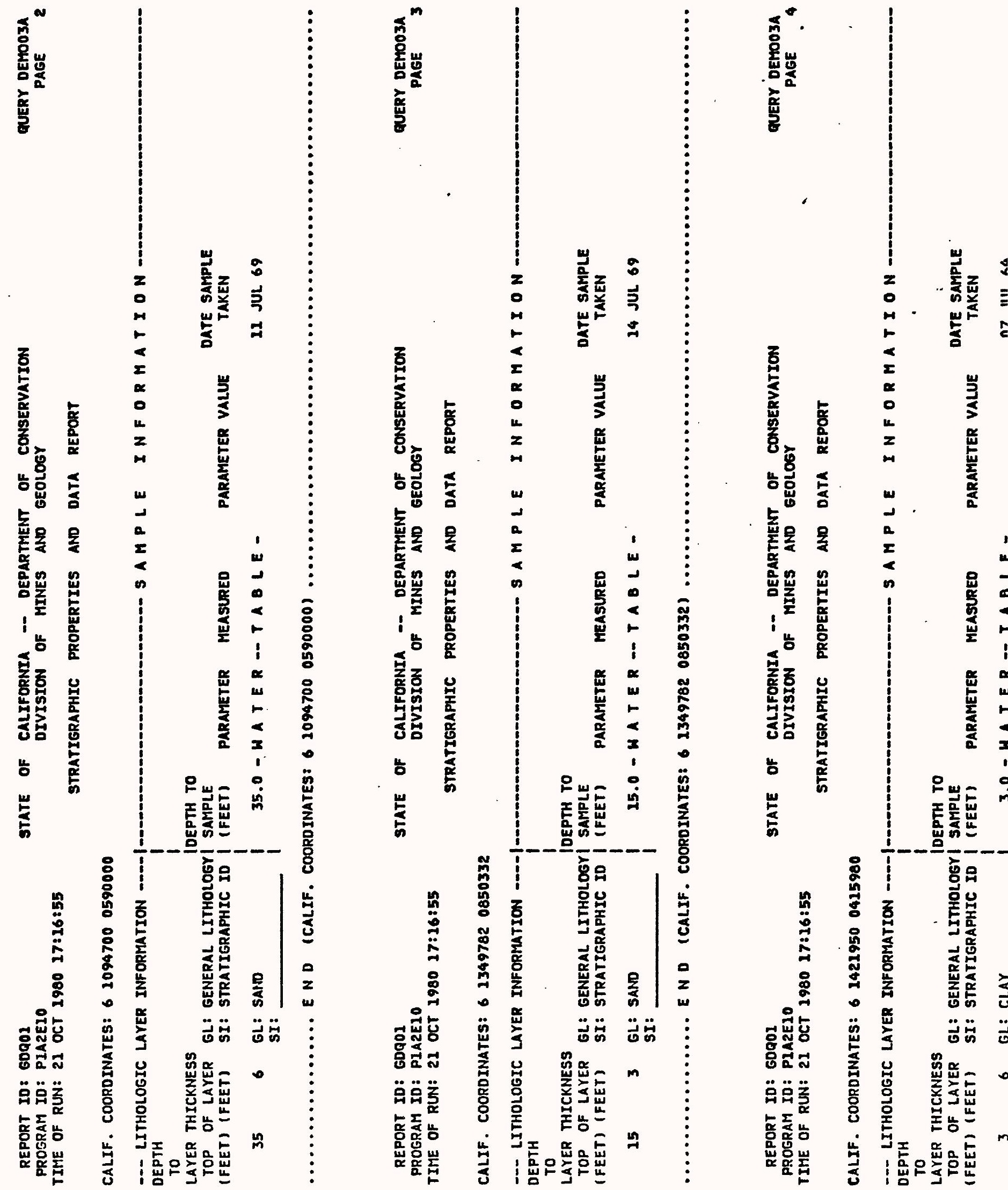
융 可

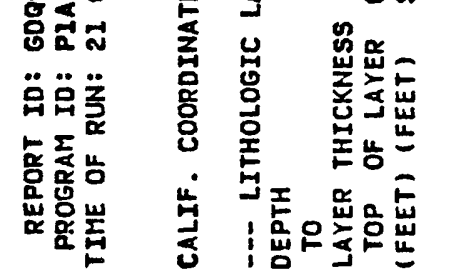




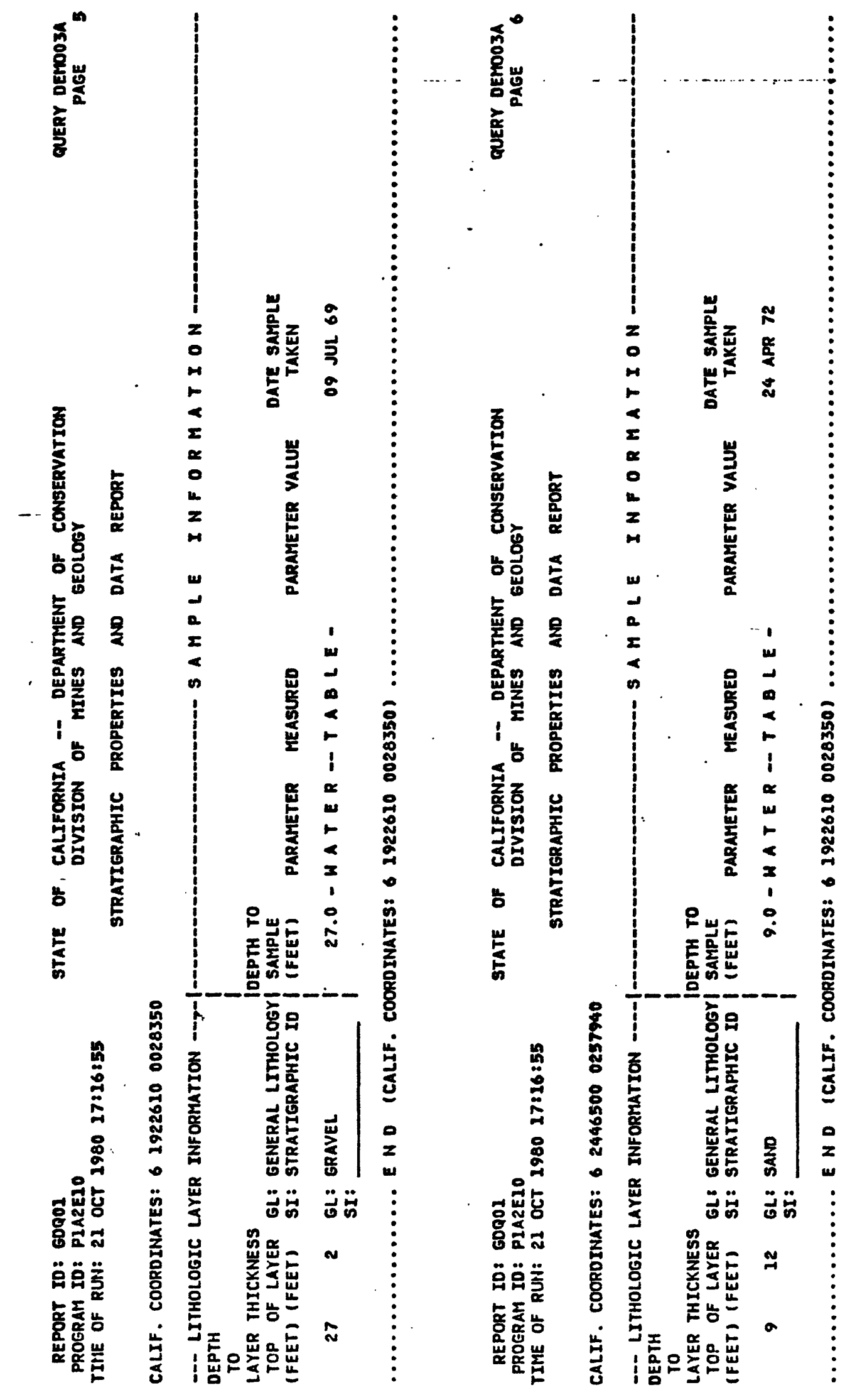



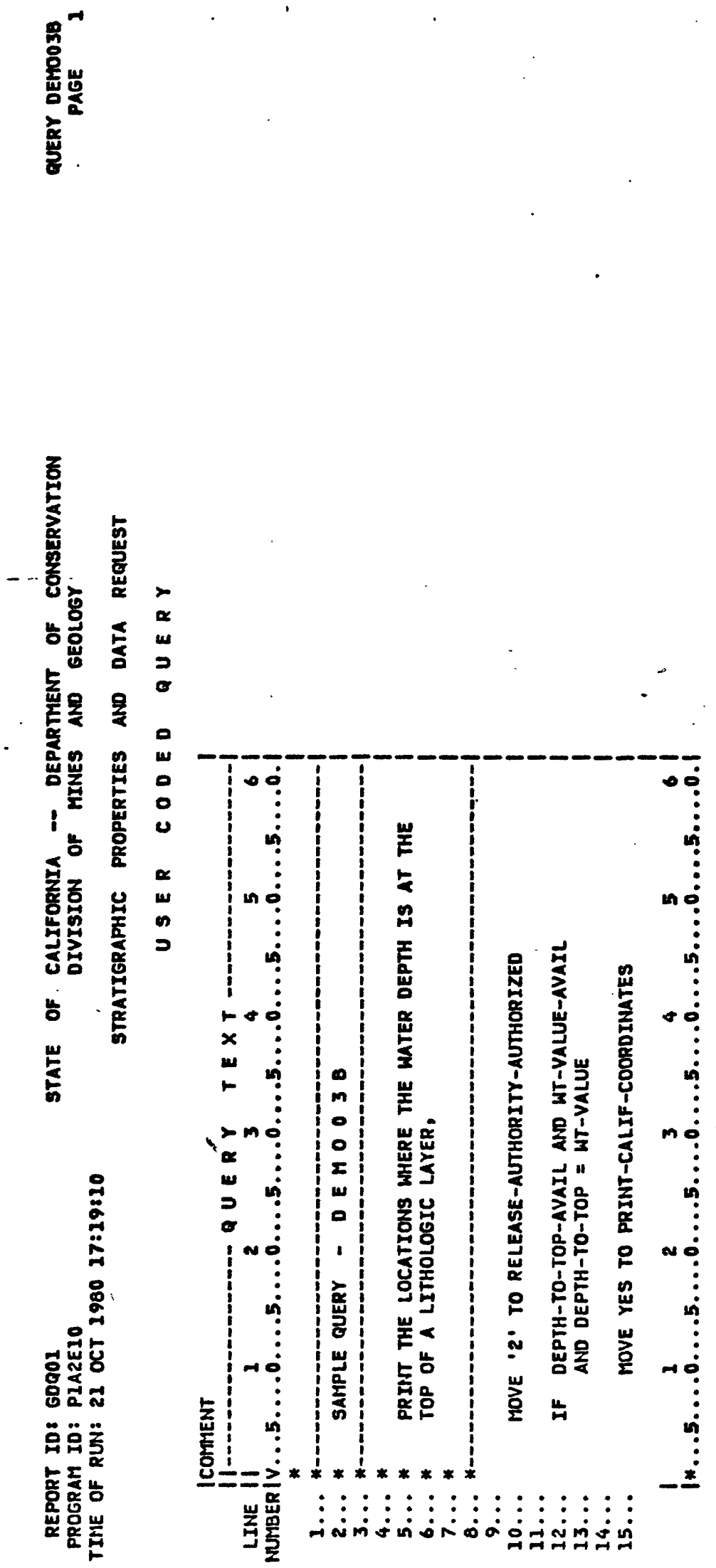


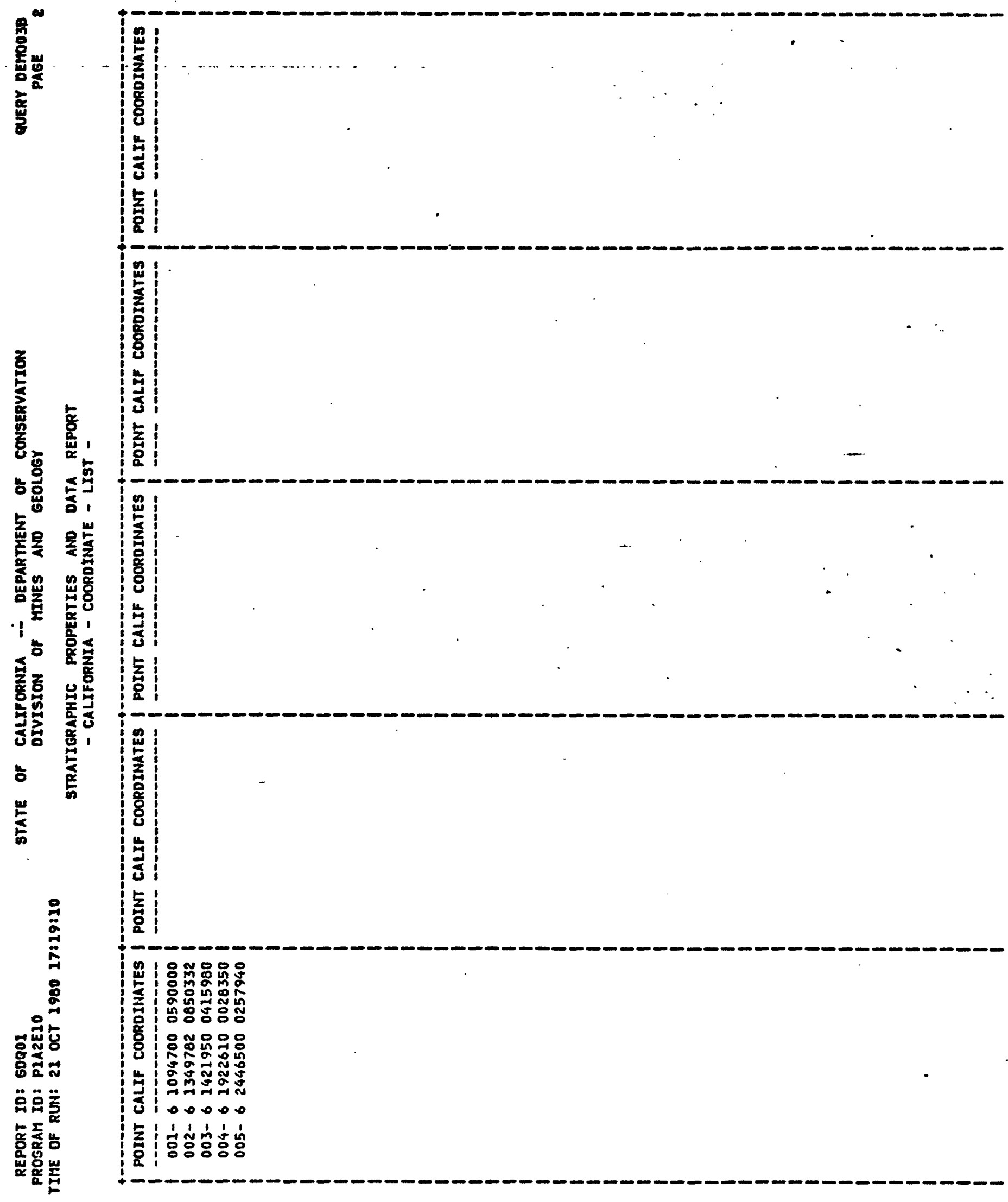



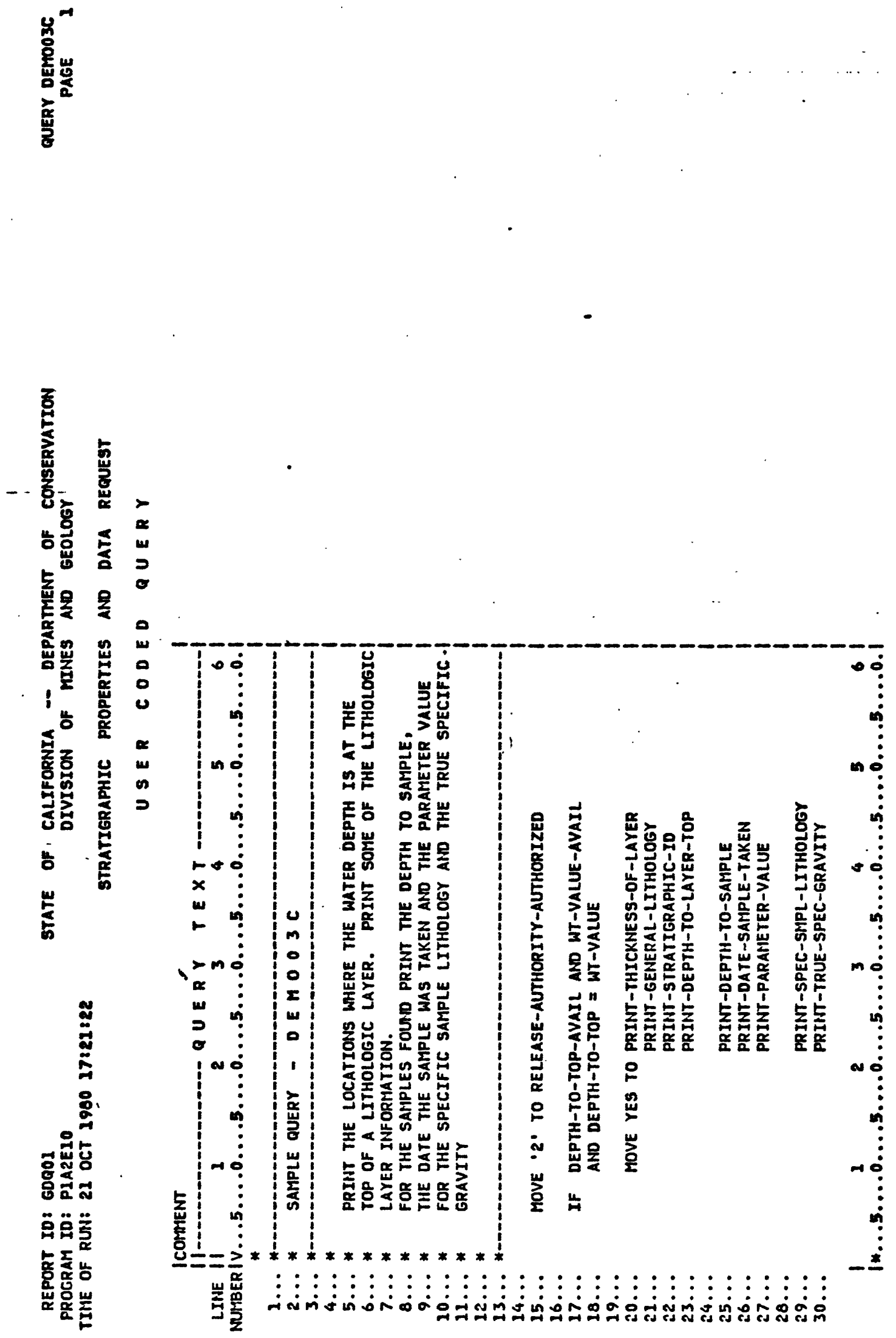

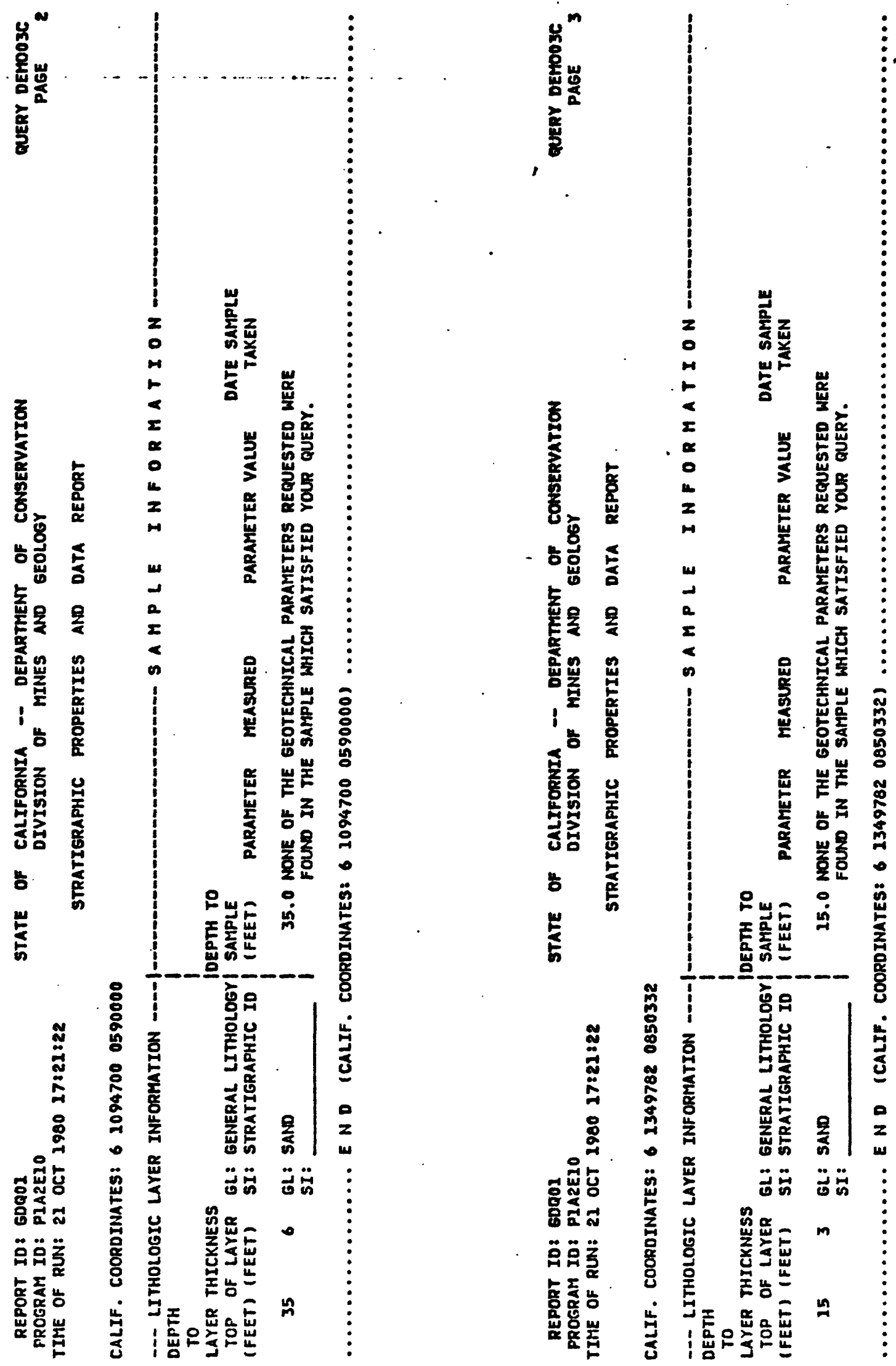

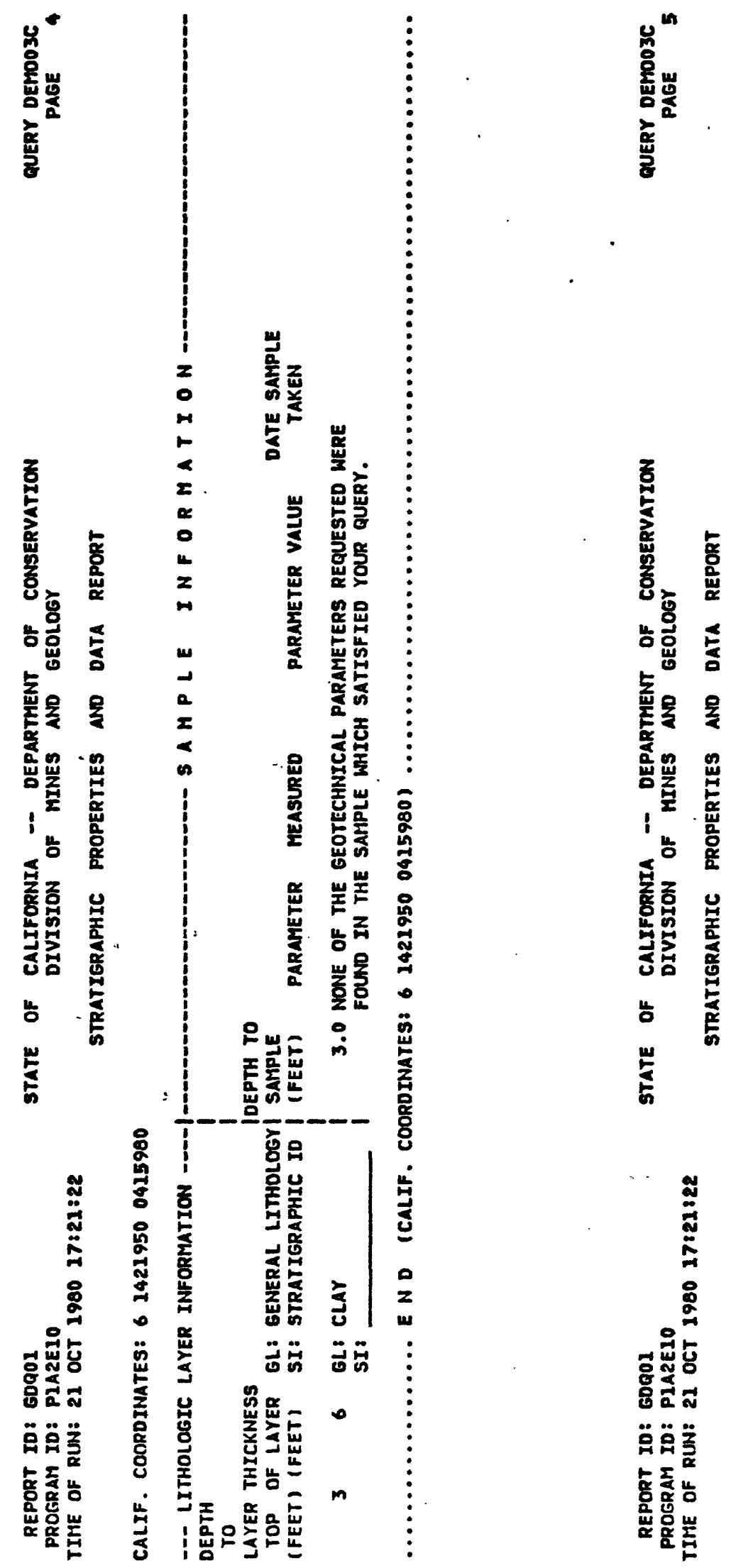

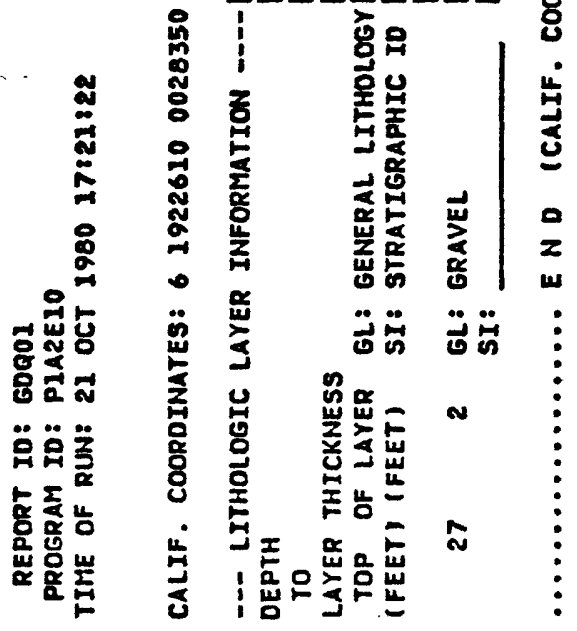




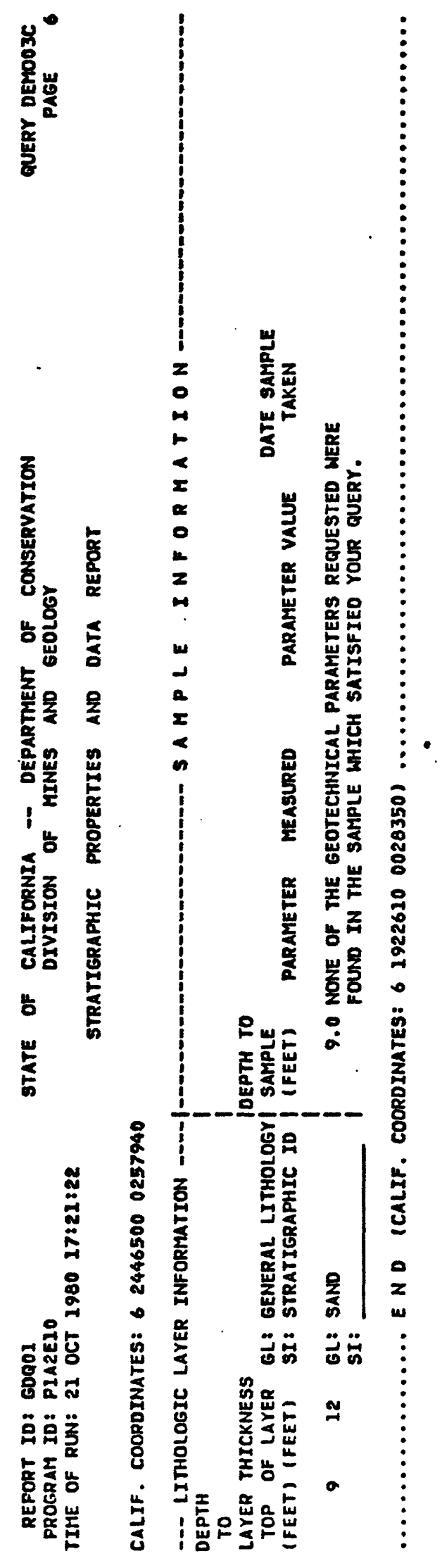



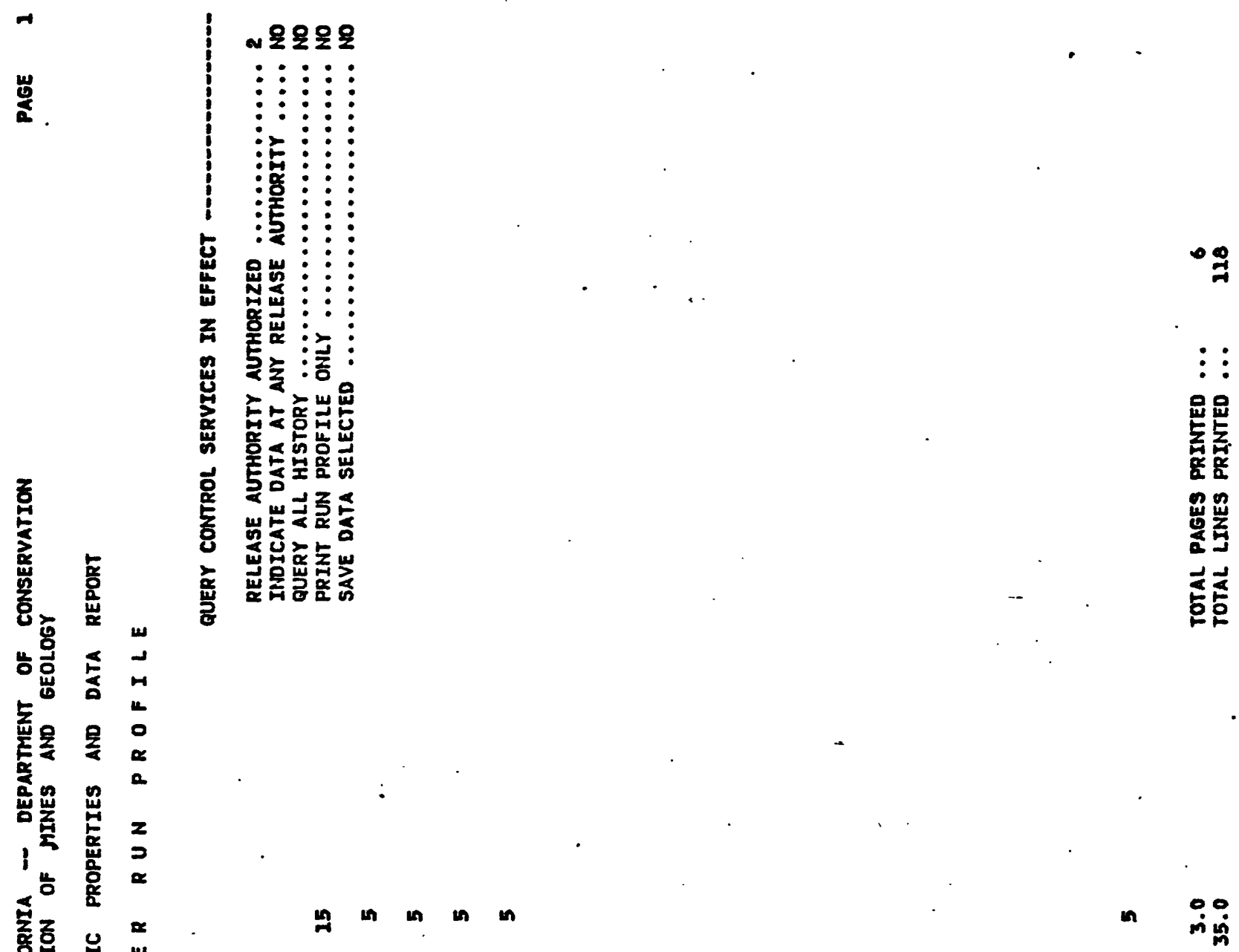

n $\dot{0}:$

"
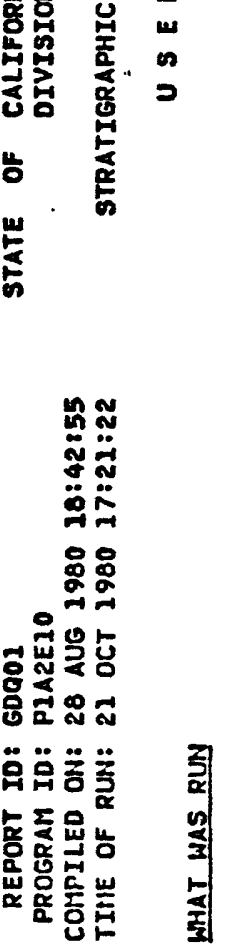

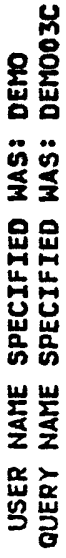

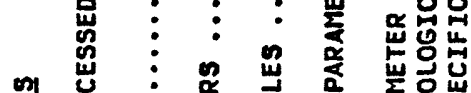

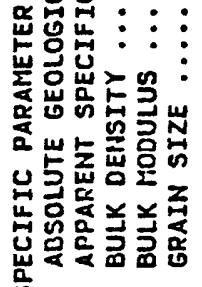

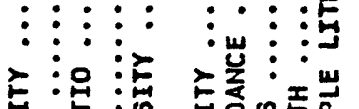

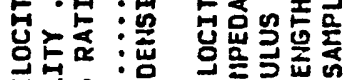

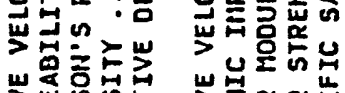

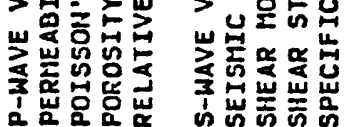

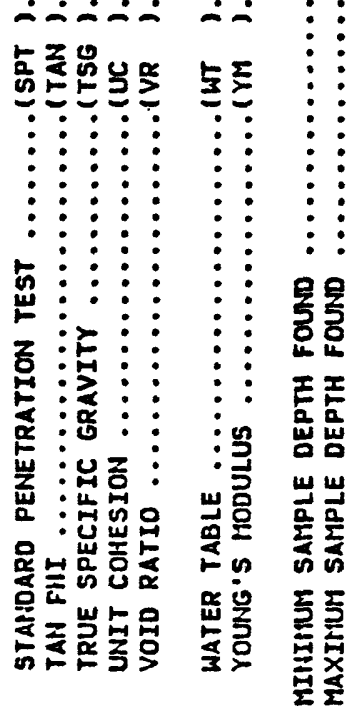

怘

능

ส

ż

ָิ

$\ddot{\text { స }}$

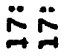

हर

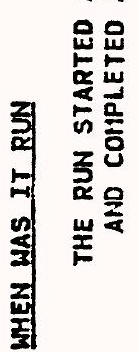




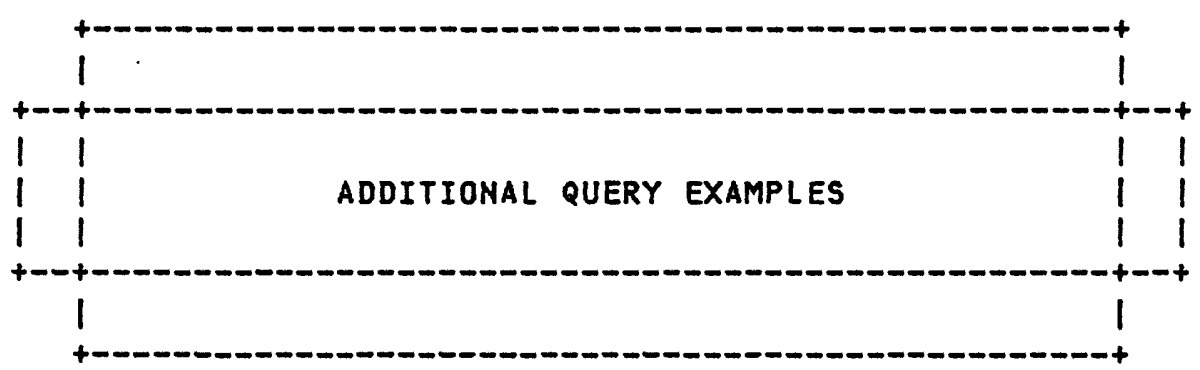




\section{QUESTION 1}

PRINT OUT THE LOCATION AND DEPTH OF ALL GROUNDWATER MEASUREMENTS AND THE DATE OF MEASUREMENT.

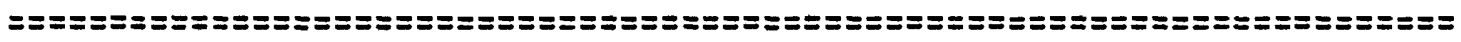

EXAMPLE 1:

*PRINT OUT THE LOCATION AND DEPTH OF ALL GROUNDWATER *MEASUREMENTS AND THE DATE OF MEASUREMENT.

MOVE ' 1 ' TO RELEASE-AUTHORITY-AUTHORIZED

IF WT-VALUE-AVAIL

MOVE YES TO PRINT-DEPTH-TO-SAMPLE

PRINT-DATE-OF-MEASUREMENT

PRINT-PARAMETER-VALUE

PRINT-WATER-TABLE-DATA 


\section{QUESTION 2}

PRINT OUT THE LOCATIONS OF ALL DATA WHERE:
A. GENERAL LITHOLOGY IS SAND
B. BULK DENSITY IS BETWEEN 90.0 AND 110.0 LB/CU FT
C. DEPTH TO SAMPLE IS LESS THAN 50 FEET
D. GROUNDWATER IS LESS THAN 10 FEET

EXAMPLE 1:

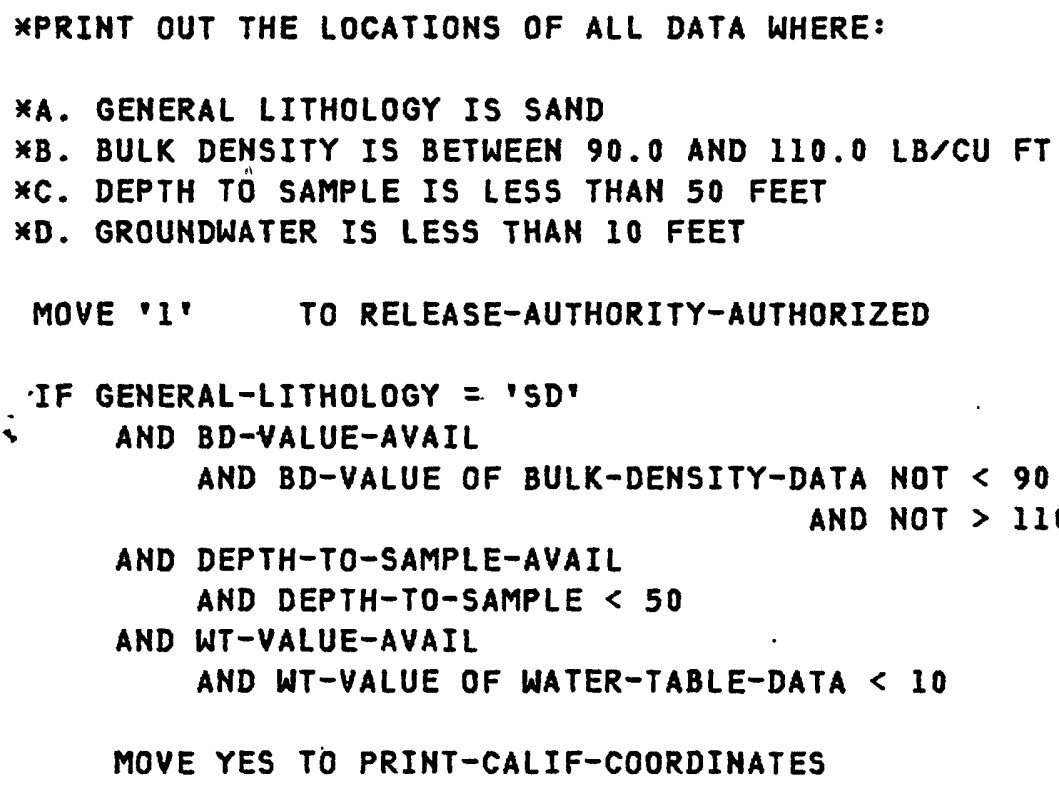




\section{QUESTION 2 (CONTINUED)}

EXAMPLE 3:

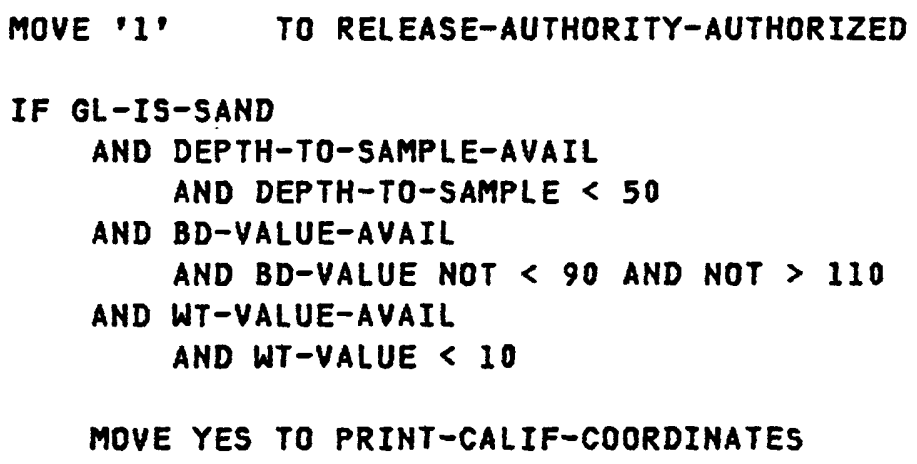




\section{QUESTION 3}

PRINT OUT THE DATA FOUND PLUS OR MINUS 1000 FEET OF THE LINE RUNNING FROM 61480,000 E 540,000N TO 6 1515,000E $624,000 N$ FOR:
A. GENERAL LITHOLOGY
B. GROUNDWATER LEVEL
C. BULK DENSITY
D. SHEAR WAVE VELOCITY
E. STANDARD PENETRATION TEST
F. SEISMIC IMPEDANCE (CALCULATED FROM BULK DENSITY AND SHEAR WAVE VELOCITY DATA)

EXAMPLE 1:

*PRINT OUT THE DATA FOUND PLUS OR MINUS 1000 FEET OF THE *LINE RUNNING FROM 6 1480,000E 540,000N TO $61515,000 E$ *624,00ON FOR:

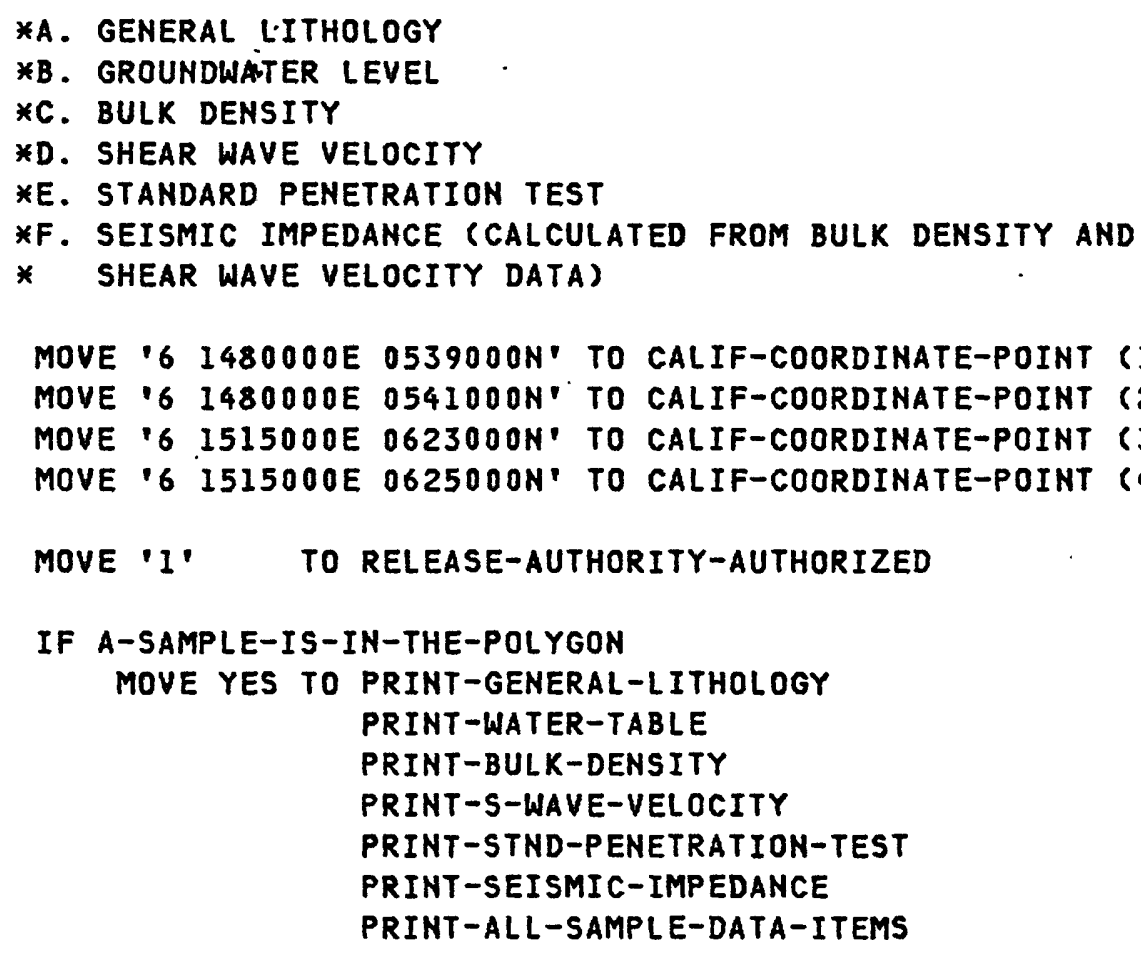




\section{QUESTION 4}

PLOT THE LOCATION AND DEPTH OF ALL VALUES OF SEISMIC IMPEDANCE DATA IN THE BASIN.

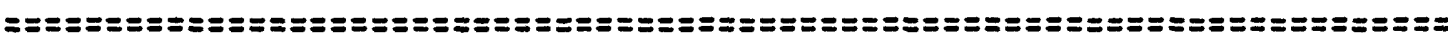

EXAMPLE 1:

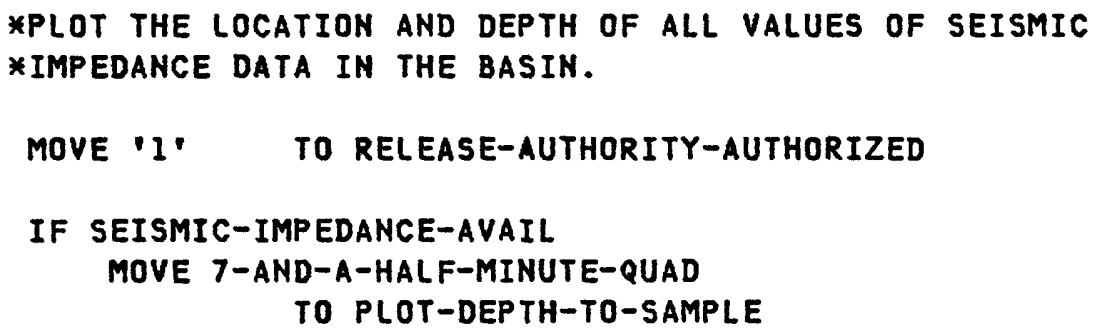

EXAMPLE 2:

*PLOT THE LOCATION AND DEPTH OF ALL VALUES OF SEISMIC *IMPEDANCE DATA IN THE BASIN.

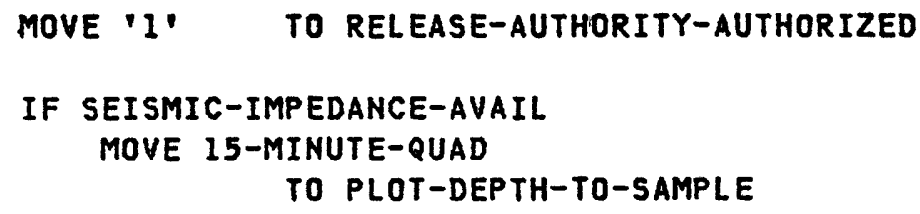




\section{QUESTION 5}

PRINT OUT ALl AVAILABLE dATA IN THE FILE.

EXAMPLE 1:

$$
\begin{array}{cl}
\text { MOVE ' } 1 " & \text { TO RELEASE-AUTHORITY-AUTHORIZED } \\
\text { MOVE YES TO } & \text { PRINT-ALL-DATA-ITEMS } \\
& \text { PRINT-SURFACE-HISTORY } \\
& \text { PRINT-LITHOLOGIC-LAYER-HISTORY } \\
& \text { PRINT-ALL-GEOTECH-PARAMETERS } \\
& \text { PRINT-SAMPLE-HISTORY } \\
& \text { PRINT-RELEASE-AUTHORITY }
\end{array}
$$

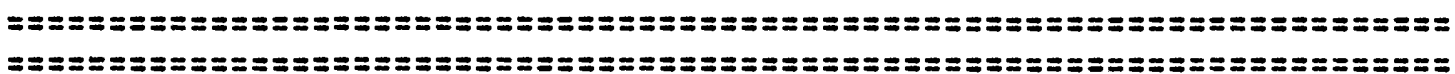

(ALTERNATE FORM FOR CURRENT DATA ONLY)

PRINT OUT ALL CURRENT DATA IN THE FILE. (I.E. NO HISTORY)

EXAMPLE 2:

$$
\begin{gathered}
\text { MOVE ' } 1 \text { ' TO RELEASE-AUTHORITY-AUTHORIZED } \\
\text { MOVE YES TO PRINT-ALL-DATA-ITEMS } \\
\text { PRINT-ALL-GEOTECH-PARAMETERS } \\
\text { PRINT-RELEASE-AUTHORITY }
\end{gathered}
$$




\section{QUESTION 6}

\section{ENGLISH QUESTION}

PRINT THE CALIFORNIA COORDINATES, SURFACE ELEVATION, DEPTH TO SAMPLE, SPECIFIC LITHOLOGY, AGE VALUE AND METHOD OF DETERMINATION FOR ALL ABSOLUTE GEOLOGIC AGE VALUES BETWEEN 500 AND 15,000 YEARS.

\section{INCORRECTLY CODED QUESTION}

IF ABSOLUTE-GEOLOGIC-AGE-AVAIL

AND ABSOLUTE-GEOLOGIC-AGE-VALUE

NOT $>15,000$ AND NOT $<500$

MOVE ' 1 ' TO RELEASE-AUTHORITY-AUTHORIZED

MOVE YES TO PRINT - CALIF. COORDINATES

PRINT - SURFACE-ELEVATION

PRINT - DEPTH-TO-SAMPLE

PRINT - SPECIFIC-LITHOLOGY

PRINT - ABSOLUTE-GEOLOGIC-AGE-VALUE

PRINT - METHOD-OF-DETERMINATION
RESPONSE

REFERENCES

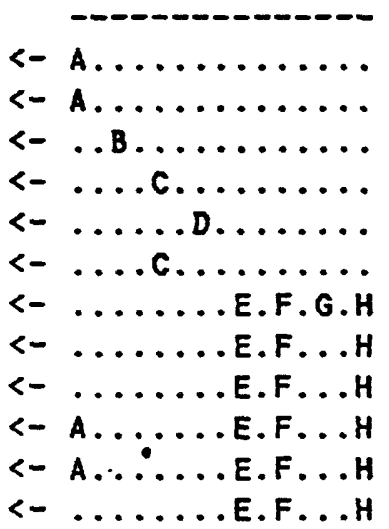

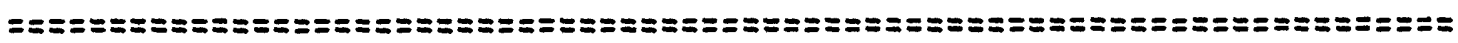

\section{CORRECTLY CODED QUESTION}

MOVE ' 1 ' TO RELEASE-AUTHORITY-AUTHORIZED

IF AGA-VALUE-AVAIL

AND AGA-AGE NOT $>15000$ AND NOT $<500$

MOVE YES TO PRINT-SURFACE-ELEVATION

PRINT-SPEC-SMPL-LITHOLOGY

PRINT-ABSOLUTE-GEOL-AGE

PRINT-DEPTH-TO-SAMPLE

PRINT-PARAMETER-VALUE

PRINT-METHOD 
RESPONSE -

A) THE QUERY PROCESSOR WILL NOT RECOGNIZE A DATA NAME OR VERB (I.E. "IF" OR "MOVE") THAT HAS BEEN SPELLED "ALMOST RIGHT".

WHERE THE QUERY PROCESSOR IS CONCERNED WHAT YOU CODE IS EITHER "RIGHT" (I.E. CORRECT) OR ELSE IT'S AN ERROR. THE QUERY PROCESSOR HAS NO UNDERSTANDING OF WHAT WE HUMANS CALL "FORGIVENESS FOR YOUR ERRORS." CODE ONLY THOSE DATA NAMES PROVIDED IN THE MENU LISTS. ALWAYS REFERENCE THE DATA NAME MENU AND THE PRINT OR PLOT SERVICE MENUS WHEN CODING YOUR QUERY. IF YOU WANT TO CHANGE THE NAMES PROVIDED IN THE MENUS, THIS CAN BE DONE.

B) DO NOT USE COMMAS WHEN CODING NUMERICAL VALUES. THE QUERY PROCESSOR DOES NOT LIKE THEM THERE, AND YOU WILL NOT LIKE THE RESULTING MESSAGES FROM THE QUERY PROCESSOR.

FOR DATA ITEMS WHOSE VALUE has a DECIMAL POINT, THE DECIMAL POINT NEED NOT BE CODED IF YOU ARE SPECIFYING AN INTEGER OF WHOLE NUMBER VALUE.

C) BLANK LINES MAY BE USED TO ENHANCE READABILITY. THE LIBERAL USE OF BLANK LINES AND "STRUCTURING" WILL ENHANCE READABILITY AND INSURE THAT THE QUESTION YOU CODE IS THE QUESTION YOU WANT TO ASK AND, MOREOVER, THAT THE DATA ITEMS YOU WANT TO BE PRINTED ARE IN FACT PRINTED.

D) THE "RELEASE-AUTHORITY-AUTHORIZED" MUST BE THE FIRST THING CODED.

E) WITH THE QUERY PROCESSOR, BLANKS ARE FINE IN SOME PLACES AND NOT FINE IN OTHER PLACES. BLANKS ARE NOT ALLOWED IN DATA NAMES. EACH DATA NAME MUST BE CODED EXACTLY AS SHOWN IN THE MENUS. AGAIN, IF YOU WANT TO CHANGE THE NAMES PROVIDED IN THE MENUS, THIS CAN BE DONE.

F) THE ORDER IN WHICH YOU CODE YOUR "PRINT" REQUESTS IN UNIMPORTANT. THE PRINT (OR PLOT) DATA NAMES MAY BE CODED IN ANY ORDER IN THE "MOVE" STATEMENT.

G) THE CALIFORNIA COORDINATES NEED NOT BE SPECIFIED TO BE PRINTED. WHY? THE CALIFORNIA COORDINATES WILL ALWAYS BE PRINTED IF ANY OTHER "PRINT" NAME IS SPECIFIED. THIS IS THE ONLY FIELD FOR WHICH THIS IS TRUE. ALL OTHER FIELDS YOU WISH TO SEE ON YOUR REPORT MUST BE SPECIFIED. (NOTE: SEE "H" BELOW.) IF YOU WISH TO SEE ONLY THE CALIFORNIA COORDINATES THEN ITS "PRINT" NAME MUST BE SPECIFIED. A SPECIAL CONDENSED FORMAT WILL BE USED TO PRINT THE CALIFORNIA COORDINATES SELECTED.

PLEASE NOTE: FOR THOSE FIELDS NOT SPECIFIED THE FIELD HEADINGS WILL ALSO NOT BE PRINTED ON YOUR REPORT. 
H) SEVERAL DATA NAMES ARE PROVIDED FOR SPECIFYING THAT ALL DATA NAMES FOR A LOGICAL DATA GROUPING SHOULD BE PRINTED (E.G. "PRINT-LITHOLOGIC-LAYER-INFO").

OTHER DATA NAMES CAN BE DEFINED WHICH IMPLY ANY COMBINATION OF FIELDS BE PRINTED. FOR EXAMPLE "PRINT-BASIC-SAMPLE-DATA-ITEMS" OR "PRINT-BASIC-GEOTECH-DATA-ITEMS" MIGHT BE DEFINED TO MEAN THAT THE DEPTH TO SAMPLE, PARAMETER VALUE, DATA OF MEASUREMENT AND METHOD ARE TO BE PRINTED. HENCE CODING

MOVE YES TO PRINT-BASIC-SAMPLE-DATA-ITEMS

OR

MOVE YES TO PRINT-BASIC-GEOTECH-DATA-ITEMS

COULD BE EQUTVALENT TO CODING

MOVE YES TO PRINT-DEPTH-TO-SAMPLE

PRINT-PARAMETER-VALUE

PRINT-DATE-OF-MEASUREMENT

PRINT-METHOD

PARTICULAR "PRINT" DATA NAMES FROM THE SURFACE OR LAYER MENUS COULD ALSO BE INCLUDED.

"GROUP" OR "COLLECTIVE" DATA NAMES MAY BE DEFINED OR ADDED AT ANY TIME ON AN AS-NEEDED OR AS-DESIRED BASIS. 